\title{
Dissection of the molecular machinery of micro- and macronucleophagy
}

\section{Dissertation}

\author{
for the award of the degree \\ "Doctor rerum naturalium" \\ of the Georg-August University Göttingen
}

within the doctoral program "Molecular Medicine" of the Georg-August University School of Science (GAUSS)

submitted by

Florian Bo Otto

from Seesen

Göttingen 2019 


\section{Members of the thesis committee and examination board}

Prof. Dr. Michael Thumm

(Supervisor and first referee)

Prof. Dr. Markus T. Bohnsack

(Second referee)

Prof. Dr. Silvio O. Rizzoli
Department of Cellular Biochemistry Center for Cellular Biochemistry University Medical Center Göttingen

Department of Molecular Biology Center for Molecular Biology University Medical Center Göttingen

Department of Neuro- and Sensory Physiology

University Medical Center Göttingen

\section{Further members of the examination board}

Prof. Dr. Stefan Jakobs

Dr. Roland Dosch

Prof. Dr. Ralph Kehlenbach
Department of NanoBiophotonics Max Planck Institute for Biophysical Chemistry Göttingen, Germany

Department of Developmental Biochemistry University Medical Center Göttingen

Department of Molecular Biology

Center for Molecular Biology University Medical Center Göttingen

Date of oral examination: 30.10.2019 


\section{Affidavit}

I hereby declare that the thesis entitled "Dissection of the molecular machinery of micro- and macronucleophagy" has been written independently and with no other sources and aids than quoted.

Florian Bo Otto

Göttingen, September 2019

Parts of this thesis will be communicated in the following publication:

Otto, F. B., \& Thumm, M. (In revision). Mechanistic dissection of macro- and micronucleophagy. Autophagy. 


\section{Table of contents}

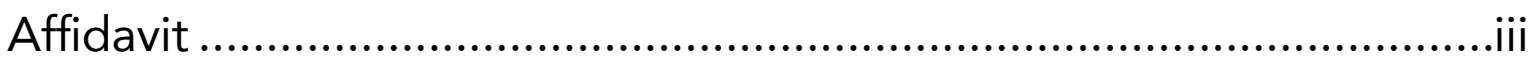

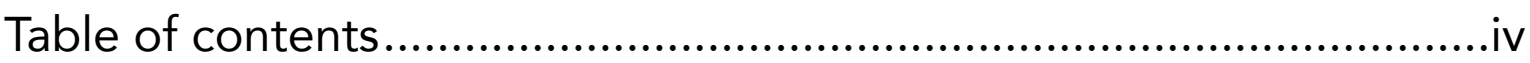

List of figures .............................................................................

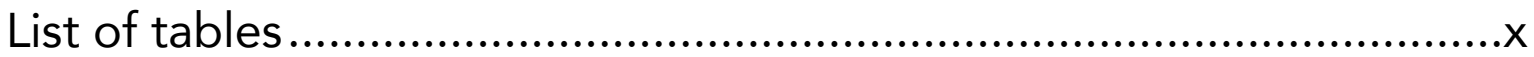

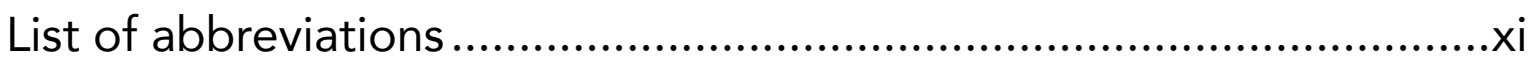

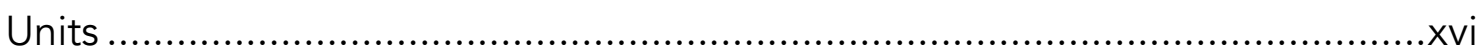

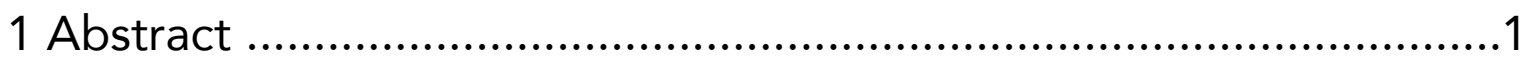

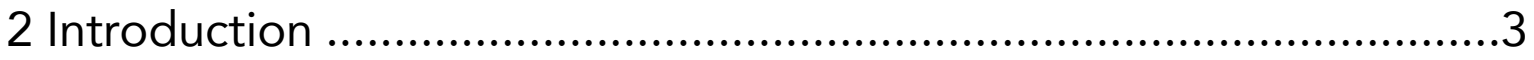

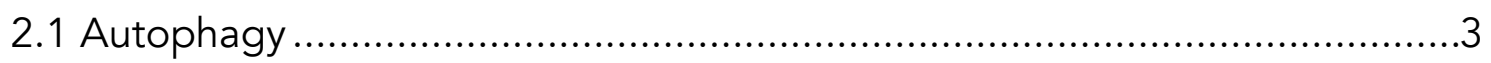

2.2 Saccharomyces cerevisiae as a model organism ........................................

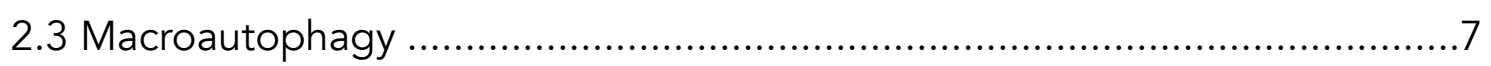

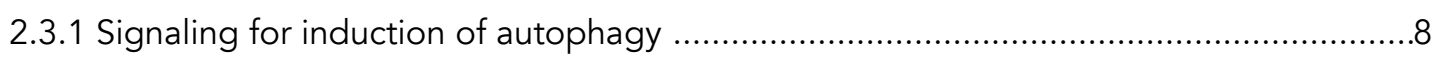

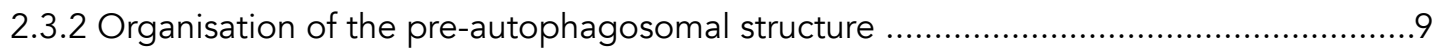

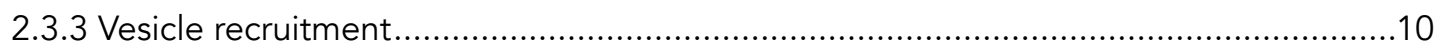

2.3.4 The phosphatidylinositol 3-kinase complex I.........................................................12

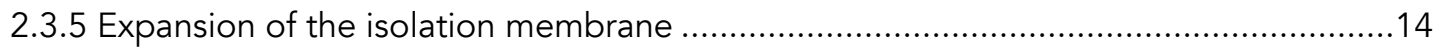

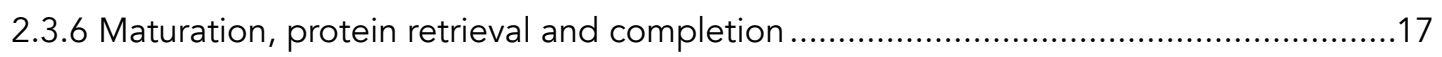

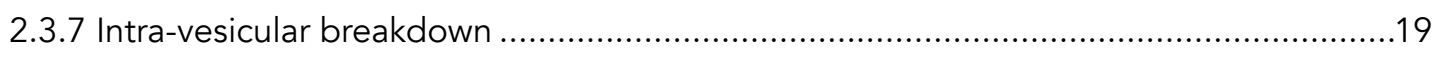

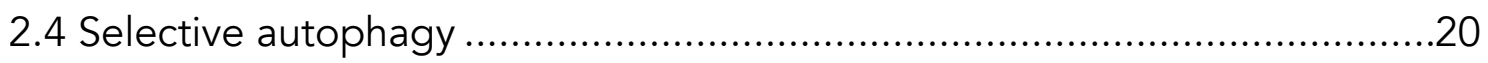

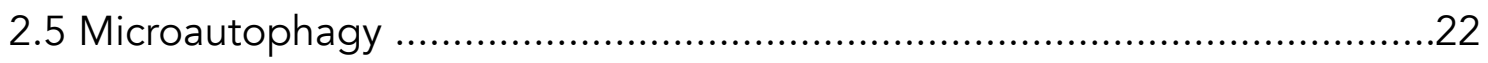

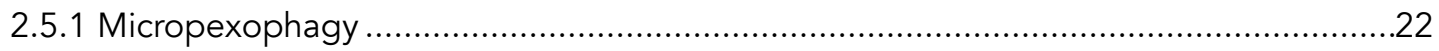

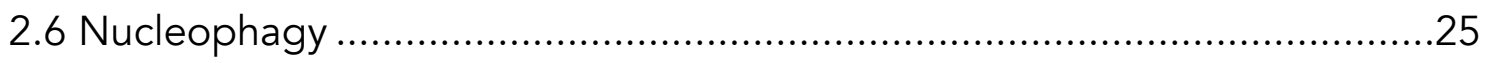

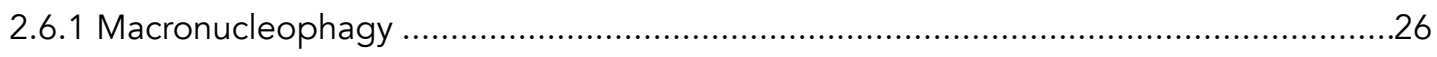

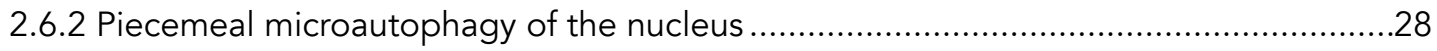

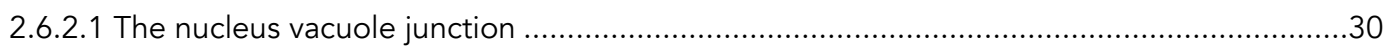




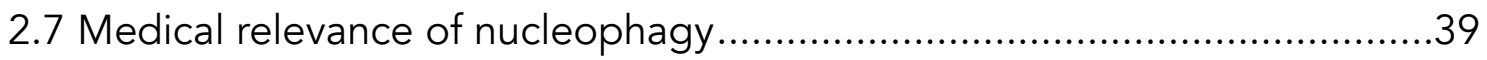

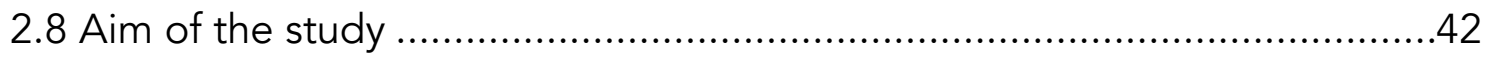

3 Materials and methods ......................................................43

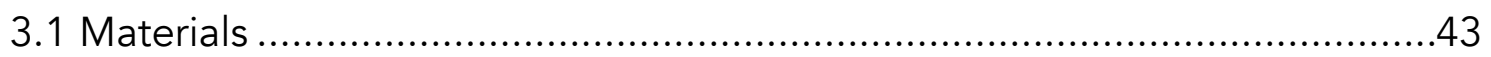

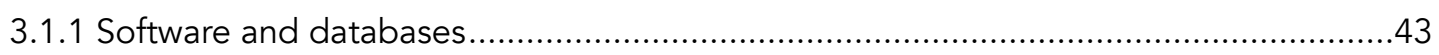

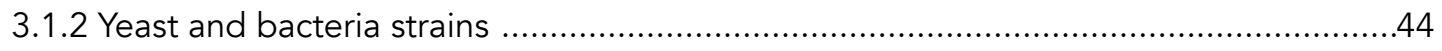

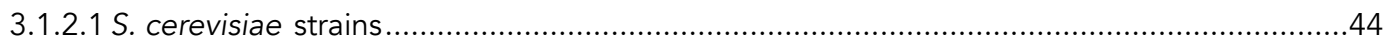

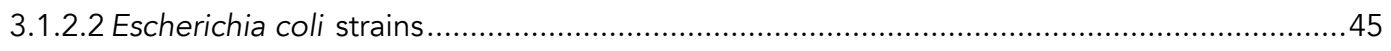

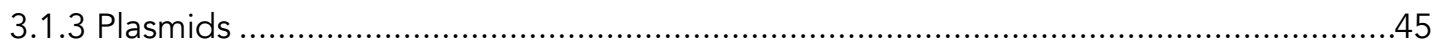

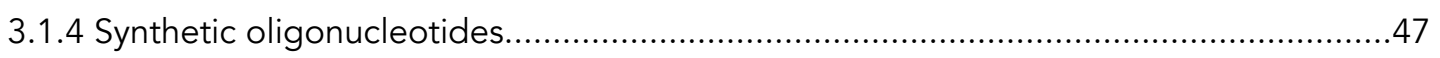

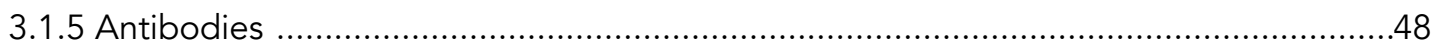

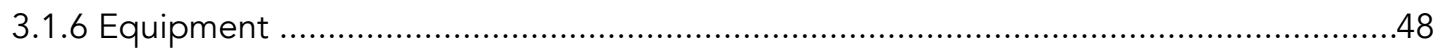

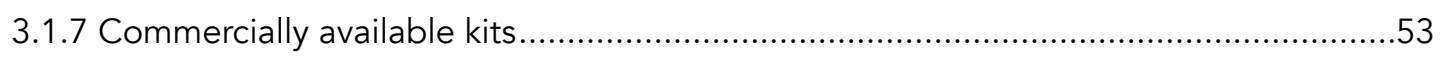

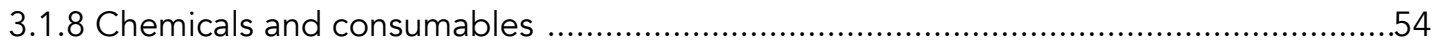

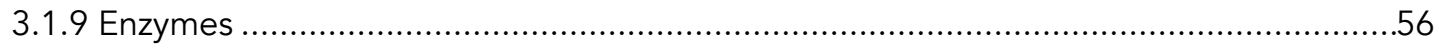

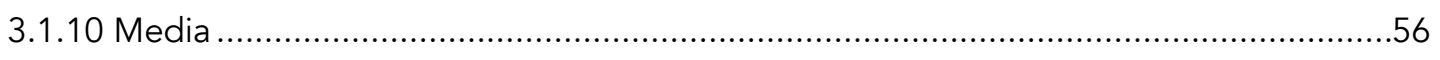

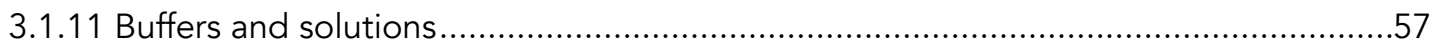

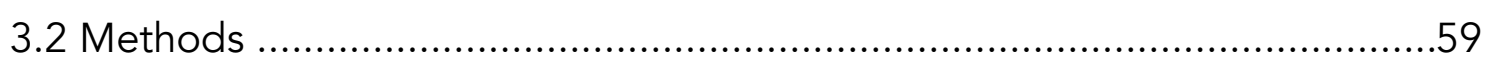

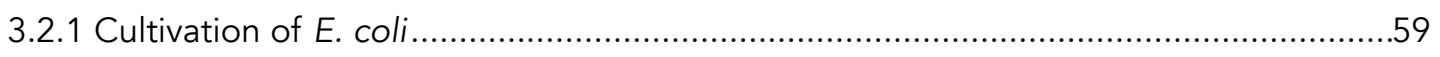

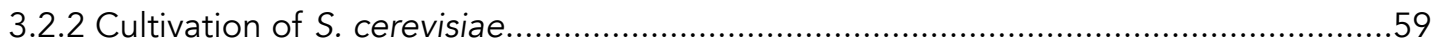

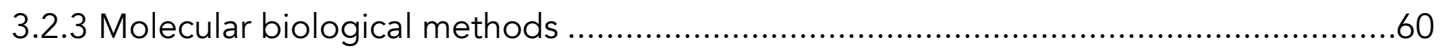

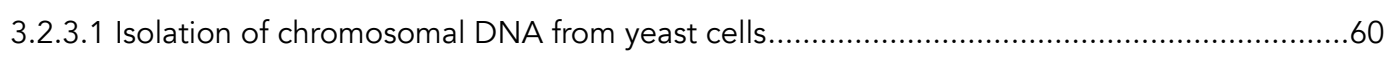

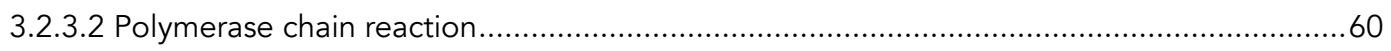

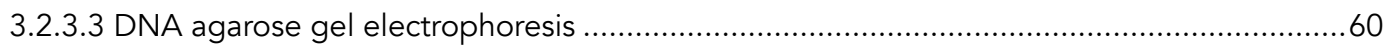

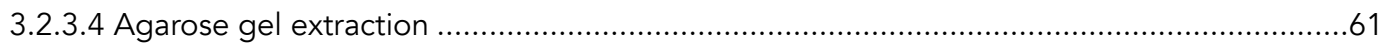

3.2.3.5 Determination and adjustment of DNA concentration ...................................................61

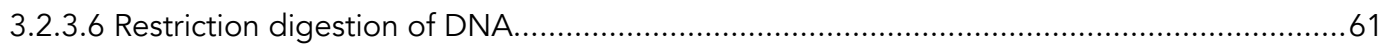

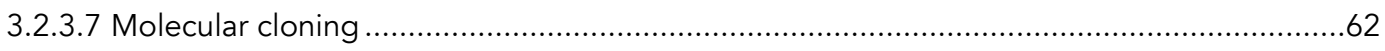

3.2.3.8 Transformation of plasmid DNA in chemically competent E. coli ......................................62

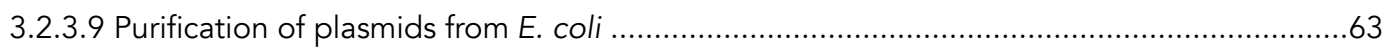




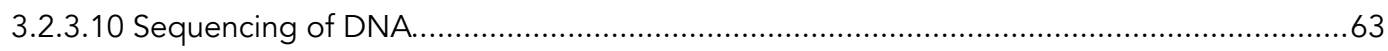

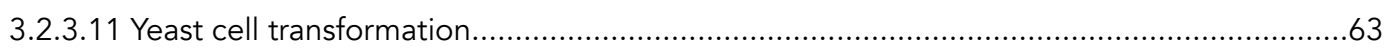

3.2.3.12 Genome engineering based on homologous recombination ...........................................64

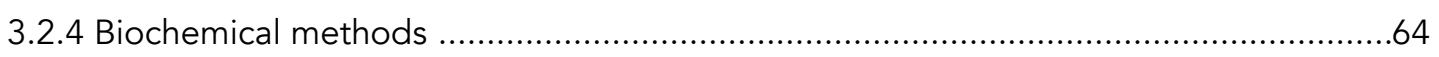

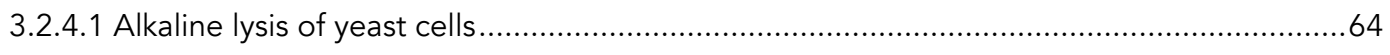

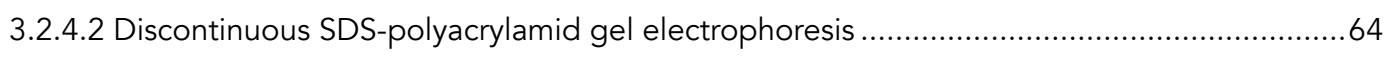

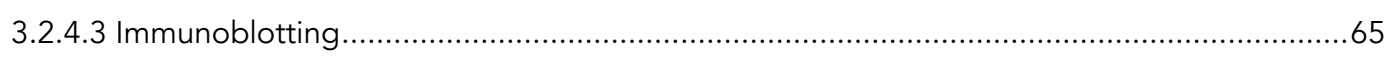

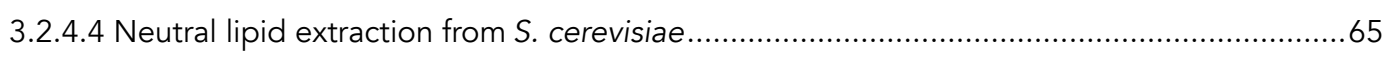

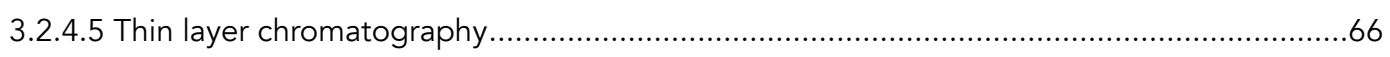

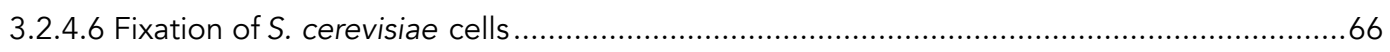

3.2.4.7 Labelling of target proteins with nanobodies in S. cerevisiae ........................................67

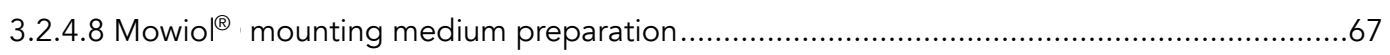

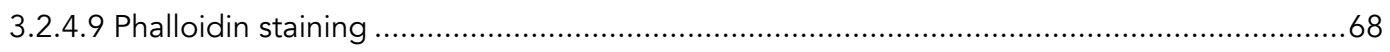

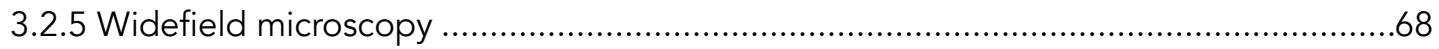

3.2.6 Confocal and stimulated emission depletion microscopy ......................................69

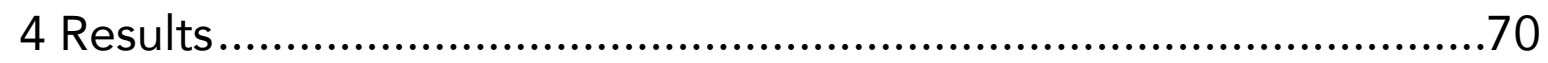

4.1 Distinction of the nucleophagic modes..............................................72

4.1.1 Macronucleophagy and PMN cargo sizes differ significantly ...............................72

4.1.2 Identification of suitable cargo for measurements regarding PMN .........................75

4.2 Microscopic characterisation of macronucleophagy ...................................76

4.2.1 Formation of autophagosomes in nucleophagic context .................................78

4.2.2 Reduction of Pho8 in the vacuolar membrane coincides with late stages of

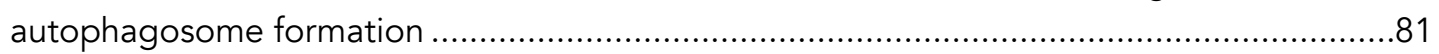

4.2.3 Localisation of the macronucleophagic cargo receptor Atg 39 ...............................83

4.2.4 The selective cargo adaptor Atg11 in macronucleophagic context ........................85

4.3 Detailing molecular mechanics of PMN ..................................................86

4.3.1 Atg8 is present between the vacuolar sequestering arms ..................................86

4.3.2 STED microscopy of autophagic structures by immuno- labelling with nanobodies....90

4.3.2.1 Establishing STED microscopy in autophagic context ...............................................91

4.3.2.2 STED microscopy of Atg8 in PMN context..................................................................92

4.3.3 The macroautophagic cargo receptor Atg39 is involved in PMN..........................93

4.3.4 Cargo adaptor Atg11 in nucleophagic context ...............................................96

4.3.5 Investigation of PI3P distribution in PMN context ............................................97 
5.1 Distinction of the nucleophagic modes.

5.1.1 Characterisation of nucleophagic cargo

5.2 Detailed investigations of nucleophagy produce novel insights into selective macroautophagy.

5.2.1 Macronucleophagy enables fine mapping of native autophagosome formation.

5.3 PMN employs a microautophagic membrane

5.3.1 The autophagic machinery at the microautophagic membrane

5.3.2 Composition of the microautophagic membrane

5.4 Atg39 assumes a central role in nucleophagy

5.4.1 Further characterisation of Atg39 in macronucleophagic context.

5.4.2 Atg39 is functionally involved in PMN

5.4.3 Atg39 links PMN to the regulation of lipid metabolism

5.5 Motor proteins are involved in the initial steps of PMN

6 Outlook

7 Bibliography.

8 Acknowledgements

9 Curriculum Vitae 


\section{List of figures}

Figure 2.1: The main subtypes of the autophagic system ...................................

Figure 2.2: Regulation of autophagic activity through TORC1 and the Atg1 kinase complex, with incorporation of Atg9-containing vesicles......................................9

Figure 2.3: The phosphoinositide 3-kinase complex | ........................................13

Figure 2.4: The two ubiquitin-like systems ………........................................15

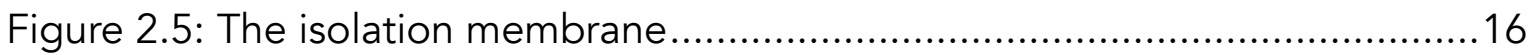

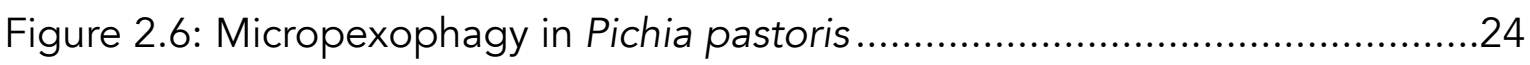

Figure 2.7: Atg39-mediated macronucleophagy ..............................................27

Figure 2.8: Piecemeal microautophagy of the nucleus........................................29

Figure 2.9: Constituents of the nucleus vacuole junction .......................................34

Figure 2.10: Additional components of nucleus vacuole contact sites .................38

Figure 4.1: Distinction of the nucleophagic modes by fluorescence microscopy .71

Figure 4.2: Cargo sizes vary between macro- and micronucleophagy ...................73

Figure 4.3: Nvj1 is degraded by PMN but not by macronucleophagy ..................75

Figure 4.4: Autophagosome biogenesis in macronucleophagic context ..............77

Figure 4.5: 3D representation of a crescent Atg8 signal ....................................78

Figure 4.6: 3D representation of a circular Atg8 signal .......................................80

Figure 4.7: 3D representation of the late macronucleophagic process..................81

Figure 4.8: Reduced quantities of Pho8 in the vacuolar membrane coincide with autohagosomal vacuolar contact sites in late stages of macronucleophagy ..........82

Figure 4.9: Localisation of Atg39 in macronucleophagic context ........................84

Figure 4.10: Atg39 accumulates around macronucleophagic cargo ......................85

Figure 4.11: Localisation of Atg11 in macronucleophagic context .......................86

Figure 4.12: Localisation of Atg8 within budding PMN vesicles ..........................87

Figure 4.13: Localisation of Atg8 in intravacuolar PMN vesicles ..........................88

Figure 4.14: No connections remain between PMN vesicle and nucleus...............89

Figure 4.15: STED microscopy of fixed and permeabilised yeast cells .................92

Figure 4.16: STED microscopy reveals a microautophagic membrane structure ..93

Figure 4.17: Localisation of Atg39 within budding PMN vesicles ........................94 
Figure 4.18: Atg39 is involved in PMN-dependent Nvj1 degradation .96

Figure 4.19: Localisation of Atg11 within budding PMN vesicles ........................97

Figure 4.20: Localisation of PI3P within budding PMN vesicles ...........................98

Figure 4.21: Visualisation of F-actin in relation to nuclear bulging.......................99

Figure 4.22: Absence of Vph1 from the NVJ suggests a sterol rich domain .......101

Figure 4.23: Lipid droplet synthesis coincides with PMN and redistribution of

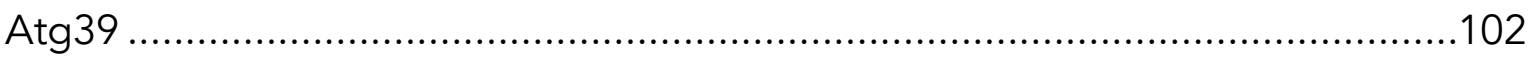

Figure 4.24: Neutral lipid extracts from PMN-disruption strains .........................103

Figure 5.1: Autophagosome biogenesis based on macronucleophagic data .....112

Figure 5.2: Integration of novel data into the model of macronucleophagy.......117

Figure 5.3: Proposed model for PMN implementing novel data ........................119 


\section{List of tables}

Table 3.1: Software and databases used in this study ...................................43

Table 3.2: Saccharomyces cerevisiae strains used in this study ...........................44

Table 3.3: Escherichia coli strains used in this study...................................45

Table 3.4: Plasmids used in this study .................................................. 45

Table 3.5: Synthetic oligonucleotides used in this study ................................47

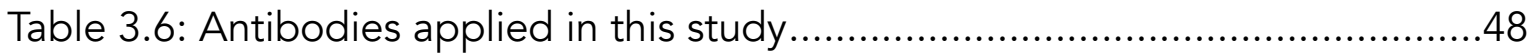

Table 3.7: Equipment used in this study............................................... 48

Table 3.8: Commercial kit solutions used in this study ..................................53

Table 3.9: Chemicals and consumables used in this study ..............................54

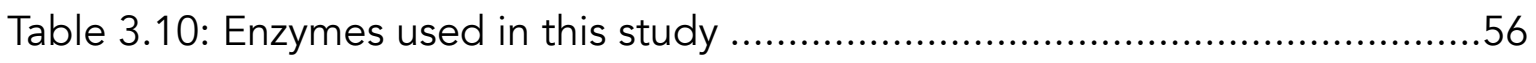

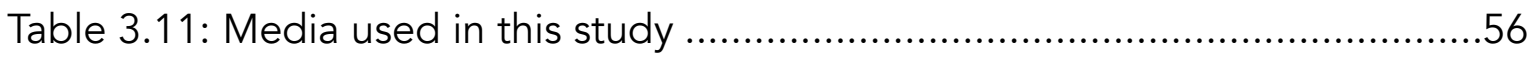

Table 3.12: Buffers and solutions used in this study ....................................57

Table 3.13: DeltaVision ${ }^{\circledR}$ filtersets applied for widefield microscopy .................68 


\section{List of abbreviations}

$\Phi:$

11-BR:

2D:

3D:

AIM:

Amp:

ANK:

AOTF:

APD:

ARM:

ATP:

BFP:

C-terminal:

C-terminus:

cAMP:

CBB:

CCD:

$\mathrm{cD}$ :

cER:

CLIP:

CM:

CMA:

COPII:

Cvt:

DAG:

$\mathrm{dd}_{2} \mathrm{O}$ :

DIC:
Hydrophobic amino acid

Atg11-binding region

Two dimensional

Three dimensional

Atg8-interacting motif

Ampicillin

Ankyrin repeat

Acousto optic tunable filter

Avalanche photodiode

Armadillo repeat

Adenosine triphosphate

Blue fluorescent protein

Carboxyterminal

Carboxyterminus

Cyclic adenosine monophosphate

Coomassie brilliant blue

Couple-charged device

Cytosolic domain

Cytosolic ER

Chromosome linkage INM protein

Complete minimal

Chaperone-mediated autophagy

Coat protein complex II

Cytoplasm-to-vacuole targeting

Diacylglycerol

Double-distilled water

Differential interference contrast 
DNA: Desoxyribonucleic acid

DTT: $\quad$ Dithiothreitol

e.g.: $\quad$ Exempli gratia; for example

EAT: $\quad$ Early autophagy targeting

EDTA: $\quad$ Ethylenediaminetetraacetic acid

EGO: $\quad$ Exit from $\mathrm{G}_{0}$

ER: $\quad$ Endoplasmatic reticulum

ERES: $\quad$ ER exit site

ERMES: $\quad$ ER-mitochondria encounter structure

ESCRT: $\quad$ Endosomal sorting complexes required for transport

EDTA: $\quad$ Ethylenediaminetetraacetic acid

et al.: $\quad$ Et alii; and others

F-actin: $\quad$ Filamentous actin

FFAc: $\quad$ Free fatty acid

FFAc: $\quad$ Free fatty alcohol

FWHM: $\quad$ Full width at half maximum

FYVE: $\quad$ Fab1, YOTB, Vac1 and EEA1

GA: Glutaraldehyde

GDP: Guanosine 5'-diphosphate

GEF: Guanine nucleotide exchange factor

GFP: $\quad$ Green fluorescent protein

GOLD: Golgi dynamics

GRAM: Glucosyltransferases, Rab-like GTPase activators and

myotubularins

GTP: Guanosine 5'-triphosphate

HOPS: Homotypic fusion and vacuole protein sorting

HORMA: Hop1, Rev7 and Mad2

HRPO: Horseradish peroxidase

Hyg: $\quad$ Hygromycin 
IDR: Intrinsically disordered region

IgG: Immunoglobulin $\mathrm{G}$

IM: Isolation membrane

INM: Inner nuclear membrane

IQR: Interquartile range

IR: Infrared

KD: $\quad$ Kinase domain

LB: $\quad$ Lysogeny broth

LD: $\quad$ Lipid droplet

$\mathrm{L}_{\mathrm{D}}: \quad \quad$ Liquid disordered

LIR: $\quad$ LC3-interacting region

LN: $\quad$ Late nucleophagy

L०: $\quad$ Liquid ordered

mCherry: $\quad$ Monomeric Cherry

MCS: $\quad$ Membrane contact site

MIM: $\quad$ MIT-interacting motif

MIP: Maximum intensity projection

MIPA: Micropexophagic apparatus

MIT: $\quad$ Microtubule-interacting and transport

MS: Mass spectrometry

mTagBFP: $\quad$ Monomeric TagBFP

MVB: $\quad$ Multivesicular body

N-terminal: $\quad$ Aminoterminal

N-terminus: $\quad$ Aminoterminus

NA: Numerical aperture

NE: $\quad$ Nuclear envelope

NGS: Normal goat serum

NLS: $\quad$ Nuclear localisation sequence

NPC: $\quad$ Nuclear pore complex 
Nrs:

Nourseothricin

NVJ:

Nucleus vacuole junction

$\mathrm{O} / \mathrm{N}$

Overnight

ONM:

Outer nuclear membrane

ORD:

OSBP-related domain

ORF:

Open reading frame

ORP:

OSBP-related protein

OSBP:

Oxysterol-binding protein

PAS:

Pre-autophagosomal structure

PBS:

Phosphate buffered saline

PCR:

Polymerase chain reaction

PE:

Phosphatidylethanolamine

PEG:

Polyethylene glycol

$\mathrm{PH}:$

Pleckstrin homology

$\mathrm{PI}:$

Phosphatidylinositol

$\mathrm{PI}(3,5) \mathrm{P}_{2}$ :

Phosphatidylinositol 3,5-bisphosphate

PI3P:

Phosphatidylinositol 3-phosphate

PI3K

Phosphoinositide 3-kinase

PI4P:

Phosphatidylinositol 4-phosphate

PKA:

Protein kinase $\mathrm{A}$

$\mathrm{PL}$ :

Phospholipid

PMN:

Piecemeal microautophagy of the nucleus

PMSF:

Phenylmethylsulfonyl fluoride

pnER:

Perinuclear ER

PROPPIN:

$\beta$-propellers that bind polyphosphoinositides

PVDF:

Polyvinylidene difluoride

PX:

Phox homology

PXA:

PX associated

PxP:

$\Phi_{x \times} \Phi_{x} P_{x} Р \Phi_{x} \Phi$ consensus containing binding motif 
RHD: Reticulon homology domain

RNA: $\quad$ Ribonucleic acid

RT:

Room temperature

SD-N:

Synthetic defined medium, lacking nitrogen

sdAb:

Single domain antibody

SDS:

Sodium dodecyl sulfate

SDS-PAGE: $\quad$ SDS polyacrylamide gel electrophoresis

SEM:

Standard error of the mean

$\mathrm{SH} 4:$

Src homology 4

SIM:

Structured illumination microscopy

SMP:

Synaptotagmin-like mitochondrial lipid-binding

SNARE:

Soluble N-ethylmaleimide-sensitive-factor attachment

receptor

SNX:

Sorting nexin

SOC:

Super optimal broth with catabolite repression

StAR:

Steroidogenic acute regulatory protein

STED:

Stimulated emission depletion

STRE:

Stress-response element

TAE:

Tris-acetate-EDTA

TAG:

Triacylglycerol

TBST:

Tris-buffered saline with Tween ${ }^{\circledR} 20$

TCA:

Trichloroacetic acid

TLC:

Thin-layer chromatography

TORC1: $\quad$ Target of rapamycin complex 1

Tris:

Tris(hydroxymethyl)aminomethane

Ubl:

Ubiquitin-like

UV:

Ultra violet

VAB:

Vps13 adaptor binding

VAP:

(Vesicle associated membrane protein) associated ER protein 
VASt:

Vad1 analog of StAR-related lipid transfer

vCLAMP:

Vacuole and mitochondria patch

VICS:

Vacuole-isolation membrane contact site

VLCFA:

Very long chain fatty acid

VSM:

Vacuolar sequestering membrane

WE:

Wax ester

WIPI:

WD repeat domain $\mathrm{Pl}$-interacting protein

w/o:

Without

w/v:

Weight per volume

$\mathrm{v} / \mathrm{v}$ :

Volume per volume

wt:

Wildtype

$x L I R:$

Extended LIR

yEGFP:

Yeast optimised, enhanced GFP

YPD:

Yeast peptone dextrose

\section{Units}

$\begin{array}{ll}{ }^{\circ} \mathrm{C}: & \text { Degree(s) celsius } \\ \text { \%: } & \text { Percent } \\ \text { × g: } & \text { Times gravity } \\ \text { A: } & \text { Ampere(s) } \\ \text { a.u.: } & \text { Arbitrary unit(s) } \\ \text { b: } & \text { Base(s) } \\ \text { bp: } & \text { Basepair(s) } \\ \text { Da: } & \text { Dalton(s) } \\ \text { g: } & \text { Gram(s) } \\ \text { h: } & \text { Hour(s) } \\ \text { Hz: } & \text { Hertz } \\ \text { L: } & \text { Litre(s) } \\ \text { M: } & \text { Mole(s) per litre }\end{array}$


min:

Minute(s)

$\mathrm{OD}_{600}:$

Optical density/absorbance at $600 \mathrm{~nm}$ wavelength

pl:

Isoelectric point

rpm:

Revolutions per minute

s:

Second(s)

V:

Volt(s) 


\section{Abstract}

A highly conserved pathway required for cellular homeostasis, autophagy facilitates degradation and recycling of various cellular components. Involvement of autophagy in medical context has made associated research a highly relevant topic for a variety of diseases and a topic of interest for the development of novel therapeutical approaches.

Nucleophagy, a functional subtype to autophagy, tasked with removal of portions of the nucleus has recently emerged as a pathway associated with various disease conditions, including neurodegenerative diseases, multiple cancer types and autoimmune diseases.

Two principal modes of nucleophagy have been described: Macronucleophagy, a selective form of macroautophagy and micronucleophagy, represented by piecemeal microautophagy of the nucleus (PMN), which involves envelopment of nuclear material, nuclear envelope and portions of the nucleolus in vacuolar arms, pinching off a portion of nucleus into the vacuolar lumen. Nuclear degradation by PMN involves a membrane contact site of nucleus and vacuole, the nucleus vacuole junction (NVJ). With the increasing evidence that supports a role for compartmental contact sites as central hubs of cellular organisation, PMN could serve as a regulatory mechanism for the NVJ that displays a unique composition and is implicated in various processes of lipid metabolism and membrane regulation.

Identification of Atg39 as the cargo receptor for perinuclear ER and the subsequent formulation of macronucleophagy has revised the perception of nucleophagy, previously thought to be represented by PMN. Parallels in cargo composition and a shared requirement for the core autophagic machinery 
prompted investigations towards differentiating and further characterising the systems that facilitate nucleophagy.

Data presented in this work suggests NVJ resident protein Nvj1 as the most reliable cargo for measuring exclusive degradation by PMN. Atg39, previously thought only to be required as the cargo receptor for macronucleophagy, was shown to be involved in PMN as well. The mechanism of PMN was further expanded by the discovery of a newly identified microautophagic membrane, present between the vacuolar arms that abstract the nuclear portion. Bulging of the nucleus, the initial step of PMN, was shown to be associated with an actin containing structure, visually reminiscent of a contractile ring. Data presented in this work supports a role for PMN as a regulatory function for the NVJ and downstream lipid metabolic processes rather than a clearance mechanism for nuclear material.

Investigations of the macronucleophagic process produced novel insights into selective macroautophagy, detailing the three dimensional morphology of autophagosome formation under native conditions and discovery of alterations in membrane composition at the vacuolar autophagosomal contact site. 


\section{Introduction}

\subsection{Autophagy}

Autophagy is a basic, catabolic pathway, regulating cellular degradation processes, that is highly conserved among eukaryotes. As a major quality control pathway, responsible for cellular homeostasis, autophagy is distinguishable from degradation by the ubiquitin-proteasome system by its versatility with regard to cargo. While proteasomal degradation generally targets short-lived proteins, autophagy is designated to the degradation of a broad spectrum of proteins, protein complexes and organelles.

Uniformly defined by delivery of its cargo to the lysosome/vacuole, autophagic mechanisms have proven to be highly diverse (Figure 2.1). Autophagy can be subdivided into three mechanistically distinct types: Macroautophagy, microautophagy and chaperone-mediated autophagy (CMA). Initially recognised as a bulk degradation pathway, autophagy was shown to be capable of functioning in a highly selective manner, in addition to its unselective modality (Gatica et al., 2018).

For macroautophagy, cargo is initially sequestered in a transient membrane structure, the isolation membrane (IM)/phagophore. The IM expands sequentially around cargo and concludes by membrane closure, resulting in formation of a double-membraned vesicle, the autophagosome. The autophagosome subsequently fuses with the lysosome/vacuole and is ultimately degraded as an autophagic body in the lysosome/vacuole (de Duve, 1963; Klionsky, 2005; Yorimitsu \& Klionsky, 2005; Saha et al., 2018). 


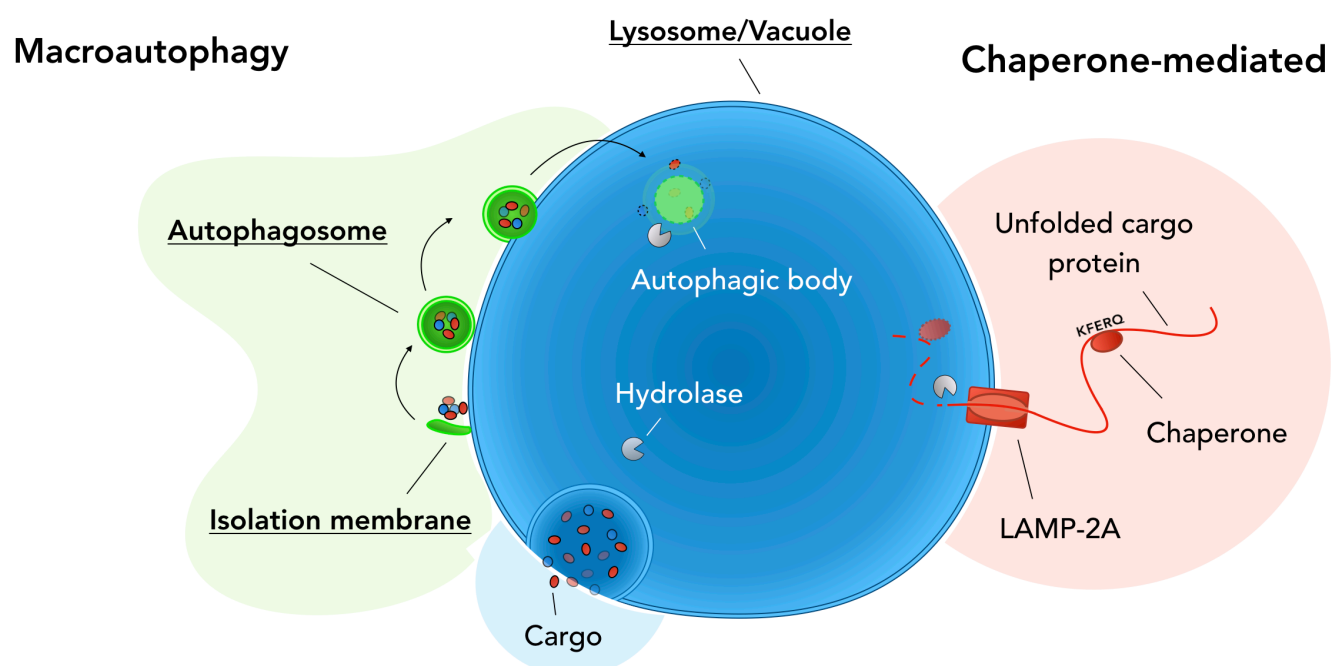

\section{Microautophagy}

\section{Figure 2.1: The main subtypes of the autophagic system}

Three main types of autophagy have been identified: Macroautophagy (green), microautophagy (blue) and chaperone-mediated autophagy (CMA; red). Macroautophagy requires the formation of a double-membrane cup-like structure, the isolation membrane which engulfs various cytosolic material. Closure of the resulting structure called autophagosome is followed by fusion of the structure with the lysosome/ vacuole, as a single-membrane autophagic body. Delivery of autophagic cargo to the lumen precedes degradation by the resident hydrolases. Microautophagy occurs by invagination of cargo by the lysosomal/vacuolar membrane, which also concludes in degradation of cargo by the lumenal hydrolases. Both autophagic types include selective modes. Exclusive to mammalian cells, CMA directly translocates unfolded proteins labelled by chaperones binding a KFERQ- or KFERQ-like motif across the vacuolar membrane in LAMP-2A-dependent manner.

Microautophagy instead abstracts cargo from the cytosol by invagination or protrusion of cargo into the lysosome/vacuole before degradation within the organelle (de Duve \& Wattiaux, 1966; Ahlberg et al., 1982;. Müller et al., 2000; Li et al., 2012).

A specialised form of autophagy, CMA is only observed in mammalian cells and represents the only type of autophagy that transports cargo proteins across the membrane through a protein-translocation complex (Cuervo \& Wong, 2014; Kaushik \& Cuervo, 2012). It is thereby, in contrast to macro- and microautophagy, 
restricted to protein degradation. Beyond its capacity for protein quality control, CMA is implicated in degradation of upstream regulators of cell death and proliferation, DNA repair and cell cycle progression, as well as constituents of cellular metabolism (Xie et al., 2015; Yang et al., 2009; Park et al., 2015; Lv et al., 2011; Xia et al., 2015). Substrates of CMA contain a pentapeptide KFERQ- or KFERQ-like motif, which is specifically recognised by chaperones, including hsc70. Binding results in unfolding of the cargo and interaction with LAMP-2A, which then multimerises into a translocation complex (Dice, 1990; Chiang et al., 1989; Bandyopadhyay et al., 2008; Rout et al., 2014; Salvador et al., 2000). Transfer across the lysosomal membrane depends on hsc70 (Agarraberes \& Dice, 2001; Cuervo et al., 1997). Like all types of autophagy, CMA concludes with degradation of its cargo by lysosomal resident hydrolases (Bandyopadhyay et al., 2010).

Experiments for this work focus on the degradation of organellar cargo, specifically macronucleophagy and a micronucleophagic mechanism termed piecemeal microautophagy of the nucleus (PMN). Investigations were therefore restricted to macro- and microautophagy, disregarding CMA. Both macro- and microautophagy have previously been investigated in Saccharomyces cerevisiae.

\subsection{Saccharomyces cerevisiae as a model organism}

The budding yeast $S$. cerevisiae remains one of the most popular eukaryotic model organisms for a broad range of molecular and cell biological research. Highly conserved biochemical pathways and a genetic homology to humans of approx. $60 \%$ have made research in S. cerevisiae relevant for other eukaryotic organisms, including mammalians and frequently impact human medical research (Gavin et al., 2002; Mager \& Winderickx, 2005; Steensels et al., 2018; Bao et al., 2018). 
With a relatively small genome $(12,000 \mathrm{~kb}$ in a haploid cell), composed of more than 6,000 open-reading frames (ORFs), few of which contain introns, on 16 chromosomes, S. cerevisiae presents a fully sequenced model system. The vast availability of data inspired the establishment of various databases dedicated to aggregating all relevant information (Goffeau et al., 1997; Mewes et al., 1997; Hughes et al., 2004; Cherry et al., 2011; McMillan et al., 2019). Beyond extensive information available for $S$. cerevisiae, a comprehensive set of molecular tools is present, allowing for convenient transformation by integration of highly established plasmid systems, simple genetic manipulation by homologous recombination, as well as providing ready-to-use plasmid and strain collections and a wide variety of assays designed for highly specific research applications (Hinnen et al., 1978; Giaever et al., 2002; Huh et al., 2003; Gelperin et al., 2005; Janke et al., 2004; Knop et al., 1999; Longtine et al., 1998; Kaplan \& Ewers, 2015; Terpitz et al., 2008). Its rapid growth rate allows for cells to duplicate within 90 min in appropriate media, and thereby provides relatively short generation times for experimental investigation (Sherman, 2002; Salari \& Salari, 2017).

Ovoid in shape and 5-10 $\mu \mathrm{m}$ in diameter, S. cerevisiae is a unicellular organism that reproduces by mitotic division, termed budding. Meiotic reproduction occurs upon sporulation of diploid cells, resulting in formation of asci, that contain four haploid ascospores. Two mating types Mata and Mat $\alpha$ were determined for haploid cells. Haploid cells of differing mating type can mate to form a diploid cell (Klar, 1987; Herskowitz, 1988; Kaeberlein, 2010).

Many key autophagic mechanisms are conserved from yeast to humans (Reggiori \& Klionsky, 2002; Delorme-Axford et al., 2015). Conservation of induction by starvation, presence of macro- and microautophagic mechanisms, as well as selective autophagy alongside systematic resemblance and genetic homology 
between components of the autophagic machinery have substantiated a universal relevance for yeast as a tool for research in the autophagic system.

\subsection{Macroautophagy}

Macroautophagy, often simply referred to as autophagy, is the most frequently investigated mode of autophagy. Initiation of macroautophagy begins at a perivacuolar section, termed the pre-autophagosomal structure (PAS). Initial growth of the cup-shaped autophagosomal precursor structure, the IM, occurs here. Expansion of the IM leads to enclosure of autophagic cargo followed by circular fusion, resulting in formation of a double-membrane vesicle, the autophagosome, which is the morphological hallmark of macroautophagy. Most essential and accessory proteins of the autophagy-related (Atg)-family involved in macroautophagy, associate with the PAS at least in a transient manner.

Of 42 identified Atg proteins in S. cerevisiae, 18 are essentially required for autophagosome formation and were thereby classified as the core autophagic machinery (Mizushima et al., 2011; Parzych et al., 2018). The core machinery can be further partitioned into six functional groups that are representative of the distinct steps required for formation of the autophagosome: The autophagy initiation complex (Atg1-kinase complex), vesicle recruiting Atg9, the autophagyspecific phosphoinositide 3-kinase (PI3K) complex I, the Atg12-Atg5 conjugation system, the Atg8-PE conjugation system and the Atg2-Atg18 complex. These six functional groups are evolutionarily conserved and localise to the PAS in a hierarchical fashion (Suzuki \& Ohsumi, 2010). 


\subsubsection{Signaling for induction of autophagy}

Induction of macroautophagy begins by regulation of PAS formation. PAS formation involves the Atg1 kinase complex (Figure 2.2A). Formation of the Atg1 kinase complex depends on regulation by two kinases: Target of rapamycin complex 1 (TORC1) and the cyclic adenosine monophosphate (cAMP)-dependent protein kinase $A(P K A)$. Both kinases are proposed to be respondent to distinct cellular conditions. TORC1 inactivation occurs following conditions of nitrogenand amino acid deprivation, as well as application of the immunosuppressant rapamycin. PKA inactivation on the other hand was shown to succeed reduced glucose levels (Noda \& Ohsumi, 1998; Budovskaya et al., 2004; Stephan et al., 2009). Active TORC1 regulates autophagy (Figure 2.2B) by phosphorylation of serine-rich Atg13, thereby interfering with the interaction to both Atg1 and Atg17 (Kamada et al., 2000; Kabeya et al., 2005; Kamada et al., 2010). PKA was shown to phosphorylate both Atg13 and Atg1 under conditions of high glucose, at sites distinct from those targeted by TORC1, yet both kinases obstruct autophagy in their active state (Budovskaya et al., 2005; Stephan et al., 2009).

Localisation of TORC1 was shown to be mediated by formation of a TORC1Gtr1/2-Ego1/2/3 complex. Gtr1 and Gtr2 interact to form a heterodimeric guanosine 5'-triphosphate (GTP)ase that either binds GTP, or guanosine 5'diphosphate (GDP), so that in case Gtr1 binds GTP, Gtr2 binds GDP and vice versa (Zhang et al., 2012; Kira et al., 2016). Depending on the current binding state, TORC1 can switch from an active to an inactive form. In case of Gtr1 binding to GTP, it renders TORC1 active. When Gtr2 is bound to GTP it renders TORC1 inactive. Gtr1 binding to GTP also coincides with TORC1 localisation throughout the vacuolar membrane, contrasting situations of Gtr2 binding to GTP, which results in punctate localisation of TORC1, suggesting potential 
regulation of autophagy by spacial means of avoiding phosphorylation by TORC1 (Kira et al., 2014; Kira et al., 2016; Binda et al., 2009).

A

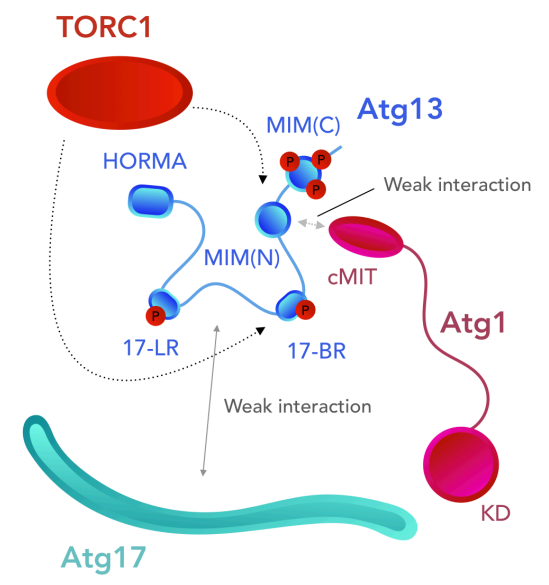

B

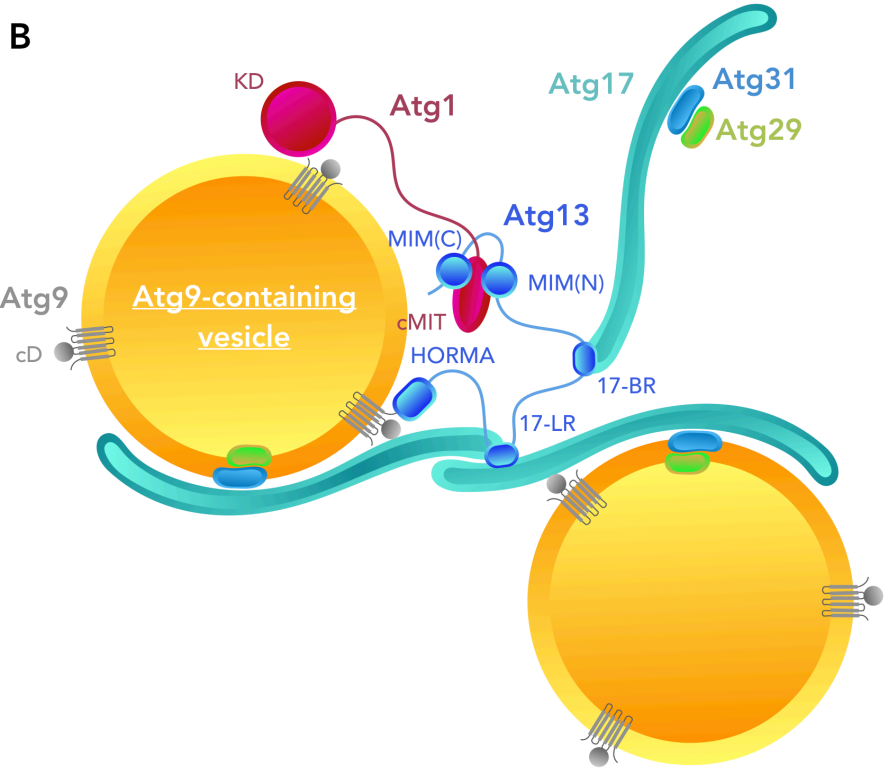

Figure 2.2: Regulation of autophagic activity through TORC1 and the Atg1 kinase complex, with incorporation of Atg9-containing vesicles

(A) Controlled by phosphorylation via active TORC1, the Atg1 kinase complex is formed under starvation conditions, inhibiting TORC1 activity. Both the Atg17-binding region (17BR) and the MIT-interacting motif (MIM) of Atg13 are directly phosphorylated by TORC1. Phosphorylation of these sites weakens interaction with Atg17 and Atg1 respectively. The weak interaction also leads to a reduction in activity of the Atg1 kinase activity, mediated by its kinase domain (KD). (B) As a result of TORC1 inhibition, the Atg1 kinase complex is formed by interaction of Atg1 and Atg13 containing the Atg17-Atg31Atg29 sub-complex. The Atg17 interacting regions (17-LR and 17-BR) allow its binding to Atg13. This complex additionally incorporates Atg9-containing vesicles. Induction of autophagy also results in phosphorylation of Atg9 by Atg1. Atg9 interacts with Atg13, mediated by the Hop1, Rev7 and Mad2 (HORMA) domain of Atg13 and with Atg17 via its cytosolic domain (cD).

\subsubsection{Organisation of the pre-autophagosomal structure}

The Atg1 kinase complex, that includes Atg1, Atg13, and the Atg17-Atg31Atg29 sub-complex (Suzuki et al., 2004; Mizushima et al., 2010), is essential for organisation of the PAS. Atg1 contains an N-terminal serine/threonine kinase domain (KD), which is linked to two carboxyterminal (C-terminal) microtubuleinteracting and transport (MIT) domains, termed early autophagy targeting (EAT) 
domain, by its intrinsically disordered region (IDR) (Matsuura et al., 1997; Fujioka et al., 2014). While its kinase functionality is required for autophagy, targeting of its interactors to the PAS occurs independent of the KD (Cheong et al., 2008; Sekito et al., 2009; Kamada et al., 2000). Autophosphorylation is facilitated by the KD and both Atg2 and Atg9 were identified as substrates to Atg1 kinase activity (Papinski et al., 2014). The EAT domain is a target for phosphorylation, but in dephosphorylated state facilitates binding to the Atg13 MIT-interacting motifs (MIM), with the Atg13 MIM-N (460-491) and MIM-C (492-521) binding in a tandem structure to the MIT domains in antiparallel fashion (Fujioka et al., 2014; Chew et al., 2015).

In addition to its MIMs, Atg13 contains an aminoterminal (N-terminal) Hop1, Rev7 and Mad2 (HORMA) domain fold, that is linked with its C-terminal IDR which harbours the MIMs. The HORMA fold interacts with the N-terminal IDR of Atg9 and also interacts directly with Atg14. Its two Atg17 binding regions (17-BR) interlink S-shaped Atg17 homodimers (Jao et al., 2013; Suzuki et al., 2015; Kabeya et al., 2009; Yamamoto et al., 2016). Each Atg17 monomer contains four $\alpha$-helices that fold into a crescent shape. Atg17 self-assembles into the S-shaped homodimers that each bind to a Atg31-Atg29 heterodimer via their C-terminal $\alpha$ helix, locating them to the concave side of its structure (Kabeya et al., 2009; Ragusa et al., 2012; Chew et al., 2013; Mao et al., 2013). Interlinking Atg17Atg31-Atg29 hexamers leads to formation of oligomerised scaffolds, composed of approx. 50 complexes (Kabeya et al., 2005; Fujioka et al., 2014; Yamamoto et al., 2016).

\subsubsection{Vesicle recruitment}

Recruitment of the transmembrane protein Atg9, delivering vesicles to the PAS, was provided as a model for membrane provision (Mari et al., 2010). Golgi- 
derived Atg9 vesicles are incorporated into the Atg1-kinase complex by binding the S-shaped homodimers of Atg17, which is theorised to enable tethering and ultimately fusion of the vesicles (Reggiori et al., 2004; Ragusa et al., 2012, Rao et al., 2016). Atg9 was identified as the sole transmembrane protein among the autophagic core proteins and contains six putative transmembrane helices flanked by IDRs on either end (Noda et al., 2000; Lang et al., 2000; He et al., 2008). Atg9 was localised on single membrane vesicles of $30-60 \mathrm{~nm}$ diameter (Yamamoto et al., 2012). Within the membrane, Atg9 is present in an oligomeric state, which is also required for efficient transport to the PAS (He et al., 2008). Apart from its interaction with Atg13, Atg9 interacts with Atg17 via its cytosolic domain (CD) and might be integral to the Atg1 kinase supercomplex (Rao et al., 2016; Sekito et al., 2009). Autophagosomes are generated de novo, and membrane provision was shown to be insufficiently provided by Atg9-containing vesicles, presuming these vesicles to only initiate nucleation at the PAS (Yamamoto et al., 2012).

COPII vesicles were implicated as a possible source. The vesicles were identified as carriers of secretory cargo out of the ER. Data showed that mutation of the inner membrane- and cargo-binding Sec23 and Sec24 subunits and also Sec12 and Sec16 COPII associated ER exit site (ERES) results in impairment of autophagy (Ishihara et al., 2001). A Rab GTPase, Ypt1, was shown to activate Hrr25, which in turn phosphorylates Sec23, leading to COPII vesicle sorting to the PAS (Wang et al., 2015). The soluble N-ethylmaleimide-sensitive-factor attachment receptor (SNARE) syntaxin protein Ufe1 of the ER was specifically shown to target COPII vesicles for recruitment towards biogenesis of autophagosomes (Lemus et al., 2016).

The guanine nucleotide exchange factor (GEF) of Ypt1, transport protein particle III (TRAPPIII), is directed to the PAS by its subunit Trs85, which binds Atg17. An 
additional interaction with Atg1 was observed as well (Wang et al., 2013). Trs85 was also shown to interact with Atg9. Tethering capacities of the Atg1-kinase complex and TRAPPIII have been hypothesised to result in heterotypic fusion of Atg9-containing- and COPII vesicles (Lynch-Day et al., 2010; Shirahama-Noda et al., 2013; Tan et al., 2013; Ge et al., 2014; Davis \& Ferro-Novick, 2015).

Determination of membrane sources for autophagy is ongoing. So far ER, Golgi, mitochondria and endosomes have all been implicated in the process of autophagosome formation (Hayashi-Nishino et al., 2009; Ylä-Anttila et al., 2009; Hailey et al., 2010; Yen et al., 2010; Itakura et al., 2012).

Phosphorylation of Atg9 leads to recruitment of the PI3K- and the Atg2-Atg18 complex (Papinski et al., 2014; Suzuki et al., 2015; Shintani et al., 2001; Wang et al., 2001). Required for retrograde transport of Atg9, both Atg1- and Atg2Atg18 complexes are thought to promote recycling of the protein (Reggiori et al., 2004; Yamamoto et al., 2012).

\subsubsection{The phosphatidylinositol 3-kinase complex I}

Enrichment with phosphatidylinositol 3-phosphate (PI3P) is a prerequisite for vesicle nucleation in autophagy (Figure 2.3). The process is mediated by the Atg14 containing, class III PI3K complex I, consisting of Vps34, Atg6 (Vps30), Vps15, Atg14 and Atg38 (Kihara et al., 2001; Araki et al., 2013). PI3P is generated by Vps34, phosphorylating the 3-hydroxyl group of the inositol ring of phosphatidylinositol (PI) (Schu et al., 1993, Stack \& Emr, 1994). The N-terminally myristoylated putative protein kinase Vps15 mediates membrane association of PI3K complexes. Vps15 myristoylation supports, but does not condition membrane binding (Herman et al., 1991). A highly conserved component, Atg6 is essential for autophagic functionality and bridged to the complex by the 
autophagy-specific component Atg14, which enables PAS localisation and is recruited by the Atg1 kinase complex components Atg13 (via its HORMA fold), Atg17 and Atg9 (Tsukada \& Ohsumi, 1993; Obara et al., 2006; Jao et al., 2013). An additional factor, Atg38 was identified and suggested to reinforce interaction of both Vps15-Vps34 as well as Atg14-Atg6 sub-complexes. Its C-terminus facilitates formation of a homodimer and an N-terminal portion contains an MIT domain, that is proposed to interact with Vps34 and Atg14. While Atg38 is not required for complex formation, its deletion leads to a significant decrease in autophagic turnover (Araki et al., 2013; Ohashi et al., 2016).

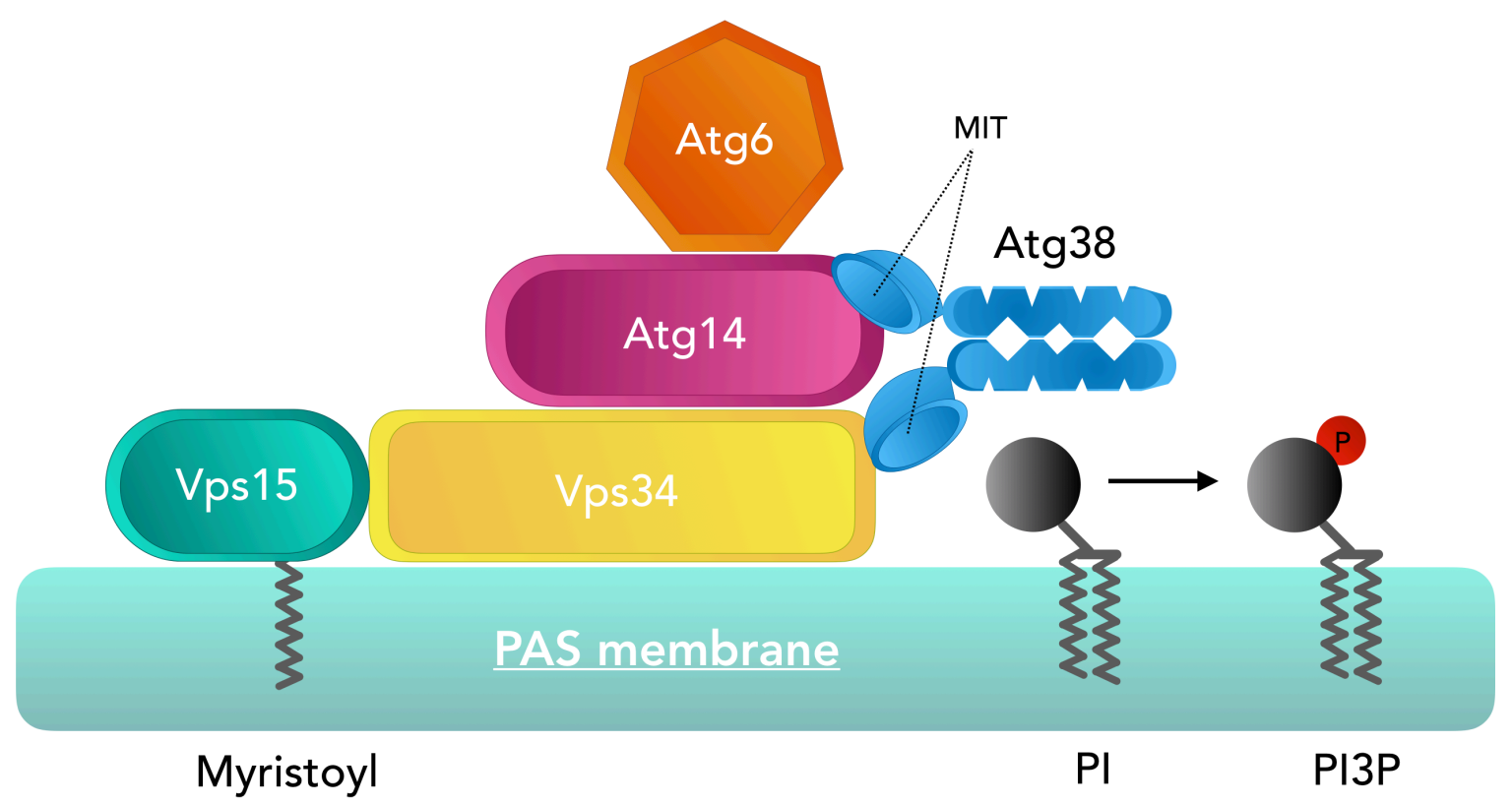

Figure 2.3: The phosphoinositide 3-kinase complex I

Required for functional autophagy, the phosphoinositide 3-kinase (PI3K) complex I consists of the PI3K Vps34, the putative kinase Vps15, which is myristoylated and Atg6 which is bridged to the complex via Atg14 which is exclusive to complex I. The Atg38 homodimer interacts with Atg14 and Vps34 via its MIT domains and is also exclusive for the PI3K complex I required for autophagy. The active PI3K complex converts phosphoinositol (PI) into phosphoinositol-3-phosphate (PI3P) resulting in enrichment of the pre-autophagosomal structure (PAS) membrane with PI3P.

Apart from autophagy, PI3P mediates cytokinesis, and is involved in downregulation of hormone receptors and endocytotic processes (Reaves et al., 
1996; Futter et al., 2001; Simonsen et al., 2001; Reidick et al., 2017). Variations of the PI3K complex are found at the endosomal membrane (as PI3K complex II), albeit in a different composition, sharing Vps34 and Vps15 with the autophagic PI3K complex I (Stack et al., 1995).

\subsubsection{Expansion of the isolation membrane}

Expansion of the IM involves two ubiquitin-like (Ubl) systems (Figure 2.4). Both systems are comprised of a Ubl protein, Atg8 and Atg12. The autophagic Ubl conjugation systems conclude in transfer of Atg8 to phosphatidylethanolamine (PE) of the IM (Ichimura et al., 2000; Hanada et al., 2007; Fujita et al., 2008).

Atg8 adopts a central role in autophagy, with many autophagic proteins, including cargo receptors, binding to Atg8 via Atg8-interacting motifs (AIMs). Atg8 is initially translated as a $13,6 \mathrm{kDa}$ protein with a C-terminal arginine. The cysteine protease Atg 4 cleaves the arginine residue of Atg8, exposing a glycine which is the initial step of the conjugation reaction (Kirisako et al., 2000). The Cterminal glycine then forms a thioester bond with the catalytic cysteine residue of the E1-like Atg7, a reaction facilitated by ATP turnover. Atg7 forms a homodimer, with one sub-unit binding to Atg8 and the other recruiting the E2-like Atg3. From this, Atg 8 is transferred to the catalytic cysteine of Atg3 by means of a thioester bond.

Contrasting Atg8, Atg12 does not require processing, due to it being translated with a C-terminal glycine. It is transferred to E2-like Atg10, which is like Atg8, mediated by Atg7. Atg12 is then by direct association of Atg10 and Atg5 transferred to the 149K residue of Atg5 (Shintani et al., 1999; Yamaguchi et al., 2012). Atg5 is an additional Ubl protein and contains two Ubl domains flanking a helix-rich domain. Its C-terminal Ubl-domain binds Atg10, yet Atg5 also binds 
Atg16. Atg16 contains an $\mathrm{N}$-terminal Atg5-binding domain and a dimeric coiledcoil domain that allows for homooligomerisation of the complex (Mizushima et al., 1999; Kuma et al., 2002; Fujioka et al., 2010; Sakoh-Nakatogawa et al., 2013).

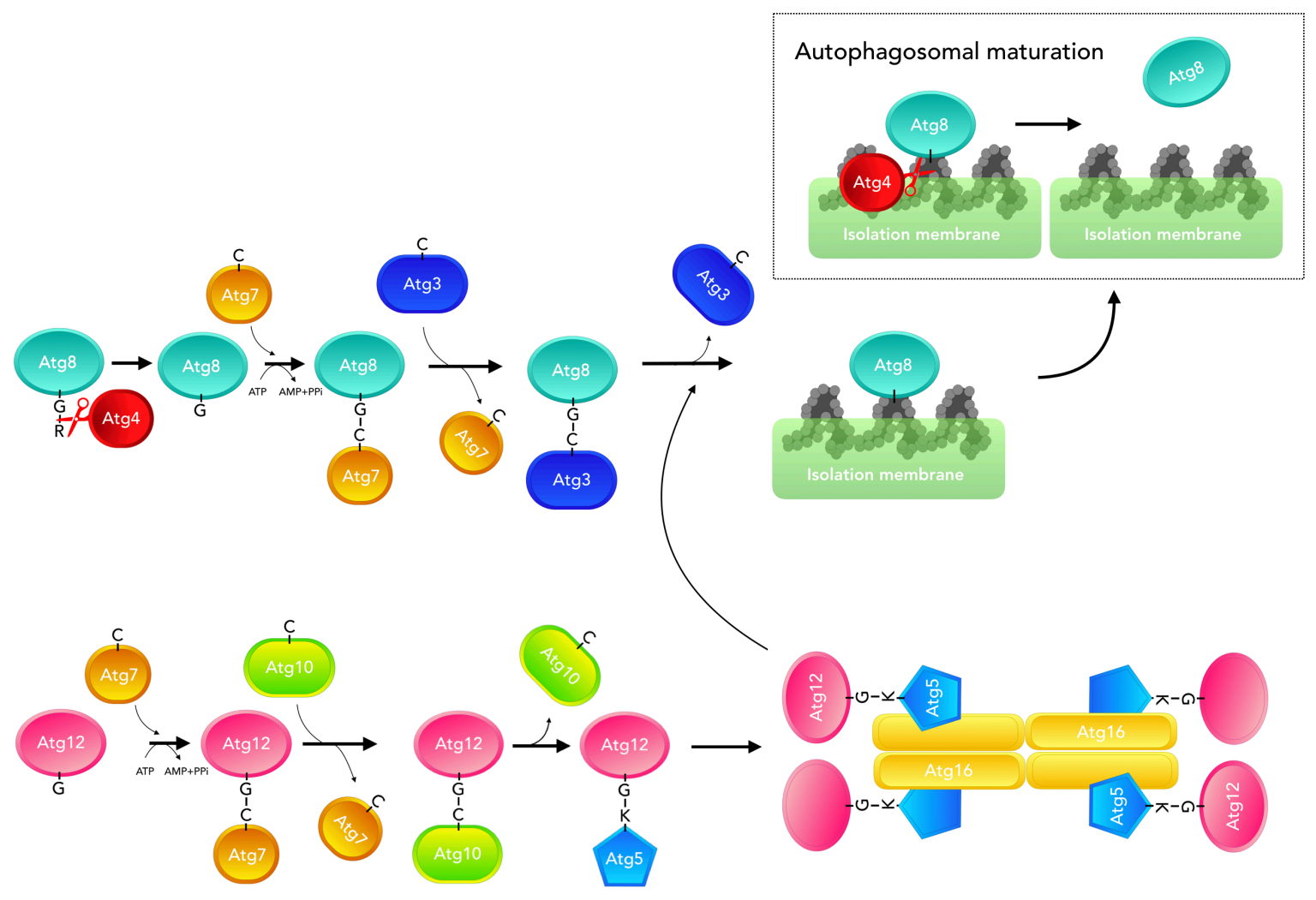

Figure 2.4: The two ubiquitin-like systems

The two ubiquitin-like (Ubl) systems of autophagy involve Atg8 and Atg12 as the Ubl proteins. Atg8 first requires removal of its C-terminal arginine by Atg4, revealing a glycine residue, required for the Ubl system. Both Atg8 and Atg12 are activated by the E1-like Atg7, forming a thioester bond of their C-terminal glycines with the catalytic cysteine of Atg7, under consumption of ATP. Atg8 and Atg12 are then transferred to the E2-like proteins Atg3 and Atg10, respectively. Atg12 is finally linked to a lysine residue of Atg5. Atg5 binds Atg16 in a non-covalent interaction, facilitating formation of a homodimeric complex, which acts as the E3-like enzyme for transfer of Atg8 from Atg3 to phosphatidylethanolamine (PE) in the isolation membrane. Upon autophagosomal maturation, Atg 4 also facilitates removal of Atg8 from PE.

The fully formed Atg12-Atg5-Atg16 complex assumes the role of an E3-like enzyme for conjugation of Atg8 to PE. Lipidation of Atg8 requires Atg16 for targeting of Atg12-Atg5 to the autophagic membrane and positioning Atg8Atg3 in close proximity of PE (Ichimura et al., 2004; Hanada et al., 2007; Suzuki et 
al., 2001; Sakoh-Nakatogawa et al., 2013). Upon interaction of Atg12 with Atg3, the Atg3 catalytic centre is rearranged, enabling transfer of the C-terminal glycine of Atg8 onto the amine moiety of PE (Ichimura et al., 2000; Hanada et al., 2007; Sakoh-Nakatogawa et al., 2013). . For its interaction with the Atg8 lipidation complex, Atg21 was proposed for determining the site of Atg8 lipidation (Juris et al., 2015)

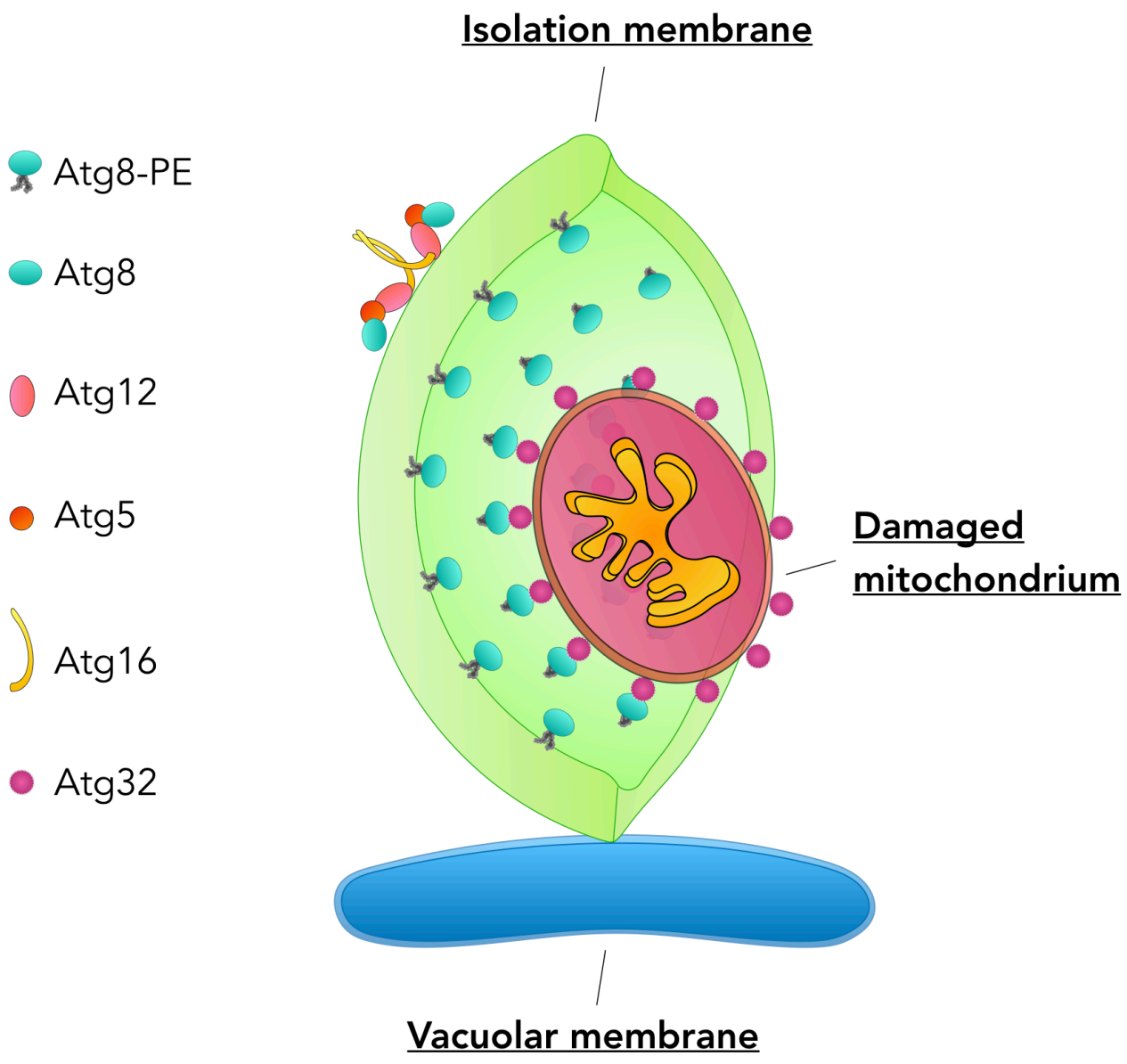

Figure 2.5: The isolation membrane

Atg8 binds the Atg12 Atg5/Atg16 complex via the cryptic Atg8-interacting motif (AIM) of Atg12. Atg16 facilitates organisation of the complex into scaffold within the convex side of the isolation membrane. Atg8 conjugated to PE is present on the concave side and enables cargo recruitment via Atg8-interacting specific cargo receptors (e.g. Atg32 for mitochondria).

Covalently bound to the autophagic membrane by PE, and present on both sides of the membrane, Atg8-PE is required for expansion of the IM and Atg8 
quantities were shown to directly correspond to autophagosomal diameter (Xie et al., 2008). Once lipidated, Atg8 anchors the Atg12-Atg5-Atg16 complex to the membrane by interaction with a noncanonical, three-dimensional AIM of Atg12. The interaction leads to the formation of a coat-like scaffold on the convex face of the IM (Kaufmann et al., 2014).

On the concave side of the IM, cargo receptors bind Atg8 by means of AIMs contained within their sequence (Figure 2.5). These interactions presumably outcompete the interaction of Atg8 with Atg12, resulting in exclusive presence of the Atg12-Atg5-Atg16 complex on the convex side of the IM (Mizushima et al., 2011; Kaufmann et al., 2014).

\subsubsection{Maturation, protein retrieval and completion}

Closure of the IM and in consequence formation of the autophagosome is not yet fully understood. Accumulation of Atg9, as well as presence of the Atg2-Atg18 complex at the tips of the IM imply a potential functionality in the closure process supported by the fact that loss of Atg2 inhibits the closure process of autophagosomes (Suzuki et al., 2013; Graef et al., 2013; Velikkakath et al., 2012). Atg8 and its mammalian LC3 counterparts were shown to mediate tethering and hemifusion by their $N$-terminal $\alpha$-helix, suggesting a potential role in either closure or fusion of autophagosomes (Nakatogawa et al., 2007; Weidberg et al., 2010; Yang et al., 2013).

Upon closure and prior to fusion with the vacuole, maturation takes place and thereby, removal of Atg family proteins and PI3P from the outer autophagosomal membrane. Removal of PI3P and Atg8 was suggested as a possible mechanism for dissociation of Atg proteins in general, yet membranes of autophagic bodies in the vacuolar lumen were shown to still contain significant amounts of PI3P 
(Parrish et al., 2004; Cebollero et al., 2012; Cheng et al., 2014). The ability of Atg4 to initially cleave Atg8 is not restricted to its native form, but also occurs for Atg8-PE, thereby releasing Atg8 from the outer autophagosomal membrane (Kabeya et al., 2000; Kirisako et al., 2000).

At least transiently present at the PAS, the PI3P phosphatase Ymr1 mediates hydrolysis of PI3P to PI (Cebollero et al., 2012; Taylor et al., 2000; Parrish et al., 2004). Deletion of Ymr1 results in accumulation of cytosolic autophagosomes that contain PI3P and Atg proteins in their outer membrane (Cebollero et al., 2012; Wu et al., 2014).

Fusion of autophagosomes with the vacuole involves the Rab GTPase Ypt7, and its corresponding GEF, the Mon1-Ccz1 complex (Kirisako et al., 1999; Wang et al., 2002; Nordmann et al., 2010). The Mon1-Ccz1 complex localises to the autophagosome and the vacuole but also to endosomes (Wang et al., 2002; Wang et al., 2003; Gao et al., 2018a). Autophagosomal targeting of the complex was shown to be dependent on a C-terminal AIM within Ccz1, whereas its recruitment of Ypt7 requires presence of PI3P (Gao et al., 2018a; Bas et al., 2018). Ypt7 further interacts with the homotypic vacuole fusion and protein sorting (HOPS) complex, functional in tethering Ypt7-positive membranes (Wang et al., 2002; Hickey \& Wickner, 2010).

The HOPS complex also invokes assembly of SNAREs, facilitating fusion of opposing membranes. Vam3, Vam7 and Vti1 at the vacuolar membrane, opposed by $Y k t 6$ at the autophagosomal membrane, are essential for the vacuolar fusion of autophagosomes (Darsow et al., 1997; Fischer von Mollard \& Stevens, 1999; Dilcher et al., 2001; Ishihara et al., 2001; Fader et al., 2009; Bas et al., 2018; Gao et al., 2018b). Atg17 interacts with Vam7 and impairment of the interaction leads to a reduction in vacuolar fusion of autophagosomes, supporting a model of 
functional overlaps between the autophagic and the fusion machinery. Similar to Atg17, Atg11 recruits Vam7 in a manner independent from the Atg1 kinase complex, yet the interaction is at least partially redundant (Liu et al., 2016; Liu \& Klionsky, 2016).

\subsubsection{Intra-vesicular breakdown}

Proteolytic processing within the vacuole is highly dependent on both Pep4 and Prb1 (Teichert et al., 1989). Efficient intra-vacuolar breakdown of autophagic bodies requires presence of Pep4, Prb1 and Atg15 (Takeshige et al., 1992; Epple et al., 2001; Teter et al., 2001).

Pep4, the aspartyl endoprotease (proteinase A) related to mammalian CTSD (cathepsin D), cleaves preferentially between hydrophobic amino acids (Ammerer et al., 1986; Woolford et al., 1986; Dreyer, 1989). The vacuolar proteinase B (Prb1) is a serine endoprotease of the subtilisin family, that has a broad substrate specificity, acting similar to porcine chymotrypsin C and trypsin (Lenney et al., 1974; Kominami et al., 1981; Moehle et al., 1987). Both proteins perform direct proteolytic processing, but are also required for activation of several other vacuolar hydrolases, including themselves and vacuolar alkaline phosphatase Pho8 (Klionsky et al., 1990; Knop et al., 1993; Van Den Hazel et al., 1996; Rupp et al., 1991; Klionsky \& Emr, 1989).

Two additional proteases, Atg42 and its functional homolog Prc1, have been proposed to be essential for breakdown of autophagic bodies. With Atg42

present, the deletion of Prc1 does not affect the degradation of autophagic bodies. Upon deletion of both Atg42 and Prc1, cells demonstrate a phenotype similar to Pep4 deletion, accumulating structures reminiscent of autophagic 
bodies and in addition defects in zymogen activation and amino acid recycling (Parzych et al., 2018).

The vacuolar phospholipase Atg15 is implicated in intra-vacuolar breakdown of autophagic bodies and cytoplasm-to-vacuole targeting (Cvt) bodies (singlemembrane intra-vacuolar vesicles that result from the Cvt-pathway) as well as turnover of lipid droplets (LDs), a substrate of Atg39-dependent microlipophagy in yeast (Epple et al., 2001; Teter et al., 2001; Vevea et al., 2015). Atg15 primarily acts towards phosphatidylserine (PS) and to lesser extent, cardiolipin and PE (Epple et al., 2001; Ramya \& Rajasekharan, 2016).

Another vacuolar feature is its acidic interior with most vacuolar hydrolases maintaining an acidic pH optimum (Nakamura et al., 1997). Mutations that impair activity of the V-ATPase, required for vacuolar acidification, show defects in protein degradation and accumulation of autophagic bodies upon nitrogen starvation, reinforcing the notion that vacuolar degradation of proteins is $\mathrm{pH}$ dependent (Nakamura et al., 1997).

\subsection{Selective autophagy}

Essential for organismal homeostasis, selective autophagy expands the autophagic system by a directed targeting system for specific cargo. Enabling adaption, regulation and maintenance of the cell, selective autophagy applies the same core autophagic machinery as non-selective autophagy. An additional set of factors convey selectivity to the system. A part of these factors and a crucial element of selective autophagy are cargo receptors. A shared feature among a majority of autophagy cargo receptors is their inherent binding capacity to Atg8/ LC3 by AIMs or LC3-interacting regions (LIRs), alongside their ability to bind cargo. 
AIMs and LIRs are often also designated the WxxL-motif (Noda et al., 2008; Birgisdottir et al., 2013). This motif was further analysed and detailed in a more differentiated consensus as $[\mathrm{W} / \mathrm{F} / \mathrm{Y}] \mathrm{xx}[\mathrm{L} / \mathrm{I} / \mathrm{V}]$. The consensus sequence is also frequently flanked by diverse sequences containing Ser, Thr and/or the negatively charged residues Glu and/or Asp (Birgisdottir et al., 2013; Rogov et al., 2014). The underlying mechanism of the AIMs/LIRs is binding of the aromatic residue $(\mathrm{W} / \mathrm{F} / \mathrm{Y})$ and the hydrophobic residue (L/I/V) to two hydrophobic pockets present in the Ubl fold of Atg8 and the LC3 family members. While functional AIM/LIR motifs mediate specific interactions, the $[\mathrm{W} / \mathrm{F} / \mathrm{Y}] \times x[\mathrm{~L} / \mathrm{I} / \mathrm{V}]$ consensus sequence is highly abundant in proteins including non-interactors of Atg8/LC3 (Kalvari et al., 2014).

As a more efficient way of predicting AIMs/LIRs, experimentally verified AIM/LIR sequences were used to define a novel consensus sequence, the extended LIR $(\mathrm{XLIR})$ sequence, which is defined as $[\mathrm{A} / \mathrm{D} / \mathrm{E} / \mathrm{F} / \mathrm{G} / \mathrm{L} / \mathrm{P} / \mathrm{R} / \mathrm{S} / \mathrm{K}][\mathrm{D} / \mathrm{E} / \mathrm{G} / \mathrm{M} / \mathrm{S} / \mathrm{T} / \mathrm{V}][\mathrm{W} / \mathrm{F} /$ $\mathrm{Y}][\mathrm{D} / \mathrm{E} / \mathrm{I} / \mathrm{L} / \mathrm{Q} / \mathrm{T} / \mathrm{V}][\mathrm{A} / \mathrm{D} / \mathrm{E} / \mathrm{F} / \mathrm{H} / \mathrm{I} / \mathrm{K} / \mathrm{L} / \mathrm{M} / \mathrm{P} / \mathrm{S} / \mathrm{T} / \mathrm{V}][\mathrm{L} / \mathrm{I} / \mathrm{V}]$, and presents a more efficient system for analysis of the highly abundant putative AIMs/LIRs (Xie et al., 2016; Kalvari et al., 2014; Jacomin et al., 2016).

Additionally, the selectivity factor Atg11, required for bridging cargo to the core autophagic machinery, was found to be essential for many types of selective autophagy, which is facilitated by Atg11-binding regions (11-BRs) within cargo receptors. Binding of Atg1 to the scaffolding Atg17-Atg31-Atg29 sub-complex is required for bulk autophagy but can be substituted for, by Atg11, that links cargo and core autophagic machinery in selective autophagy. A straightforward distinction between selective and bulk autophagy by determining requirement for either Atg11 or Atg17 remains difficult, since multiple autophagic types require both proteins (Kawamata et al., 2008; Liu et al., 2016). 


\subsection{Microautophagy}

In the microautophagic process, cargo is taken up into the vacuole by invagination of the vacuolar membrane. Microautophagy is very poorly characterised in comparison to macroautophagy. Shown to target ER and parts of the nucleus, LDs, mitochondria and peroxisomes, microautophagic systems generally share the core autophagic machinery. Microlipophagy and microautophagy of the ER were shown to require only a select subset (Kiššová et al., 2007; Roberts et al., 2003; Kvam \& Goldfarb, 2007; Schuck et al., 2014; Soubannier et al., 2012; Habibzadegah-Tari \& Dunn, 2004; van Zutphen et al., 2014; Wang et al., 2014; Vevea et al., 2015).

As for macroautophagy, induction of microautophagy was observed by nitrogen starvation or application of rapamycin. Controlled by the TORC1 and EGO signalling complexes, microautophagy elicits uptake and degradation of vacuolar membrane (Uttenweiler et al., 2007). Microautophagy was also proposed to be a mechanism for compensation of extensive membrane influx generated by vesicular fusion processes at the vacuole such as macroautophagy. Microautophagy could decrease vacuolar membrane, reducing abstraction and degradation of cytosolic content to a mere side-effect of the underlying regulatory process (Levine \& Klionsky, 2004).

\subsubsection{Micropexophagy}

A particularly comprehensive investigation of microautophagy was performed for autophagic degradation of peroxisomes in Pichia pastoris (Figure 2.6). When changing carbon sources in media from methanol, that induces peroxisome generation, to glucose, the additional peroxisomes are rendered redundant and micropexophagy is observed as a consequence (Tuttle \& Dunn, 1995). Micropexophagy combines mechanisms from both models of macro- and 
microautophagy. While peroxisome clusters are engulfed in vacuolar extensions, named vacuolar sequestering membranes (VSMs), that were shown to septate, the tips are linked by a crescent shaped membrane, reminiscent of the macroautophagic IM. Subsequently denominated micropexophagic apparatus (MIPA), this crescent shaped membrane was uniquely observed for the micropexophagic process in P. pastoris (Mukaiyama et al., 2004; Oku et al., 2003).

As a selective process, micropexophagy in $P$. pastoris is mediated by cargo receptor PpAtg30, represented by Atg36 in S. cerevisiae, that binds the two peroxisomal membrane proteins PpPex3 and PpPex14 (Farré et al., 2008; Farré et al., 2013). Like Atg36 in S. cerevisiae and in line with its designated receptor attribution, PpAtg30 binds both PpAtg8 and selectivity factor PpAtg11, which resides on the rim of the VSM (Farré et al., 2013; Motley et al., 2012; Oku et al., 2006). In addition to this interaction, PpAtg30 also interacts with PpAtg17 (Farré et al., 2013). Binding of PpAtg30 to PpAtg11 was shown to be conditioned by PpPex3. PpPex3 not only recruits PpAtg30 but also enables its phosphorylation, thereby facilitating binding to PpAtg11 (Burnett et al., 2015).

While the vacuolar membrane protein PpVac8 was shown to be required for micropexophagy, it is dispensable for macropexophagy (Fry et al., 2006; Oku et al., 2006). PpVac8 disruption impairs localisation of PpAtg11 between vacuole and VSM, the site that accumulates the PI3P-binding sorting nexin (SNX) PpAtg24, which is required for vacuole fusion (Oku et al., 2006; Ano et al., 2005).

The MIPA contains both PpAtg8 and PpAtg26 and its formation was shown to specifically require PpAtg35 which forms a complex with PpAtg17 by interaction with PpAtg28 (Mukaiyama et al., 2004; Oku et al., 2003; Nazarko et al., 2011; Stasyk et al., 2006). While both PpAtg35 and PpAtg28 are dispensable for macropexophagy, PpAtg17-PpAtg28-PpAtg35 complex assembly appears to be 
micropexophagy specific. Of note, complex assembly was shown to be localised at the nuclear envelope (NE), potentially facilitated by the C-terminal nuclear localisation sequence (NLS) of PpAtg35 (Nazarko et al., 2011).

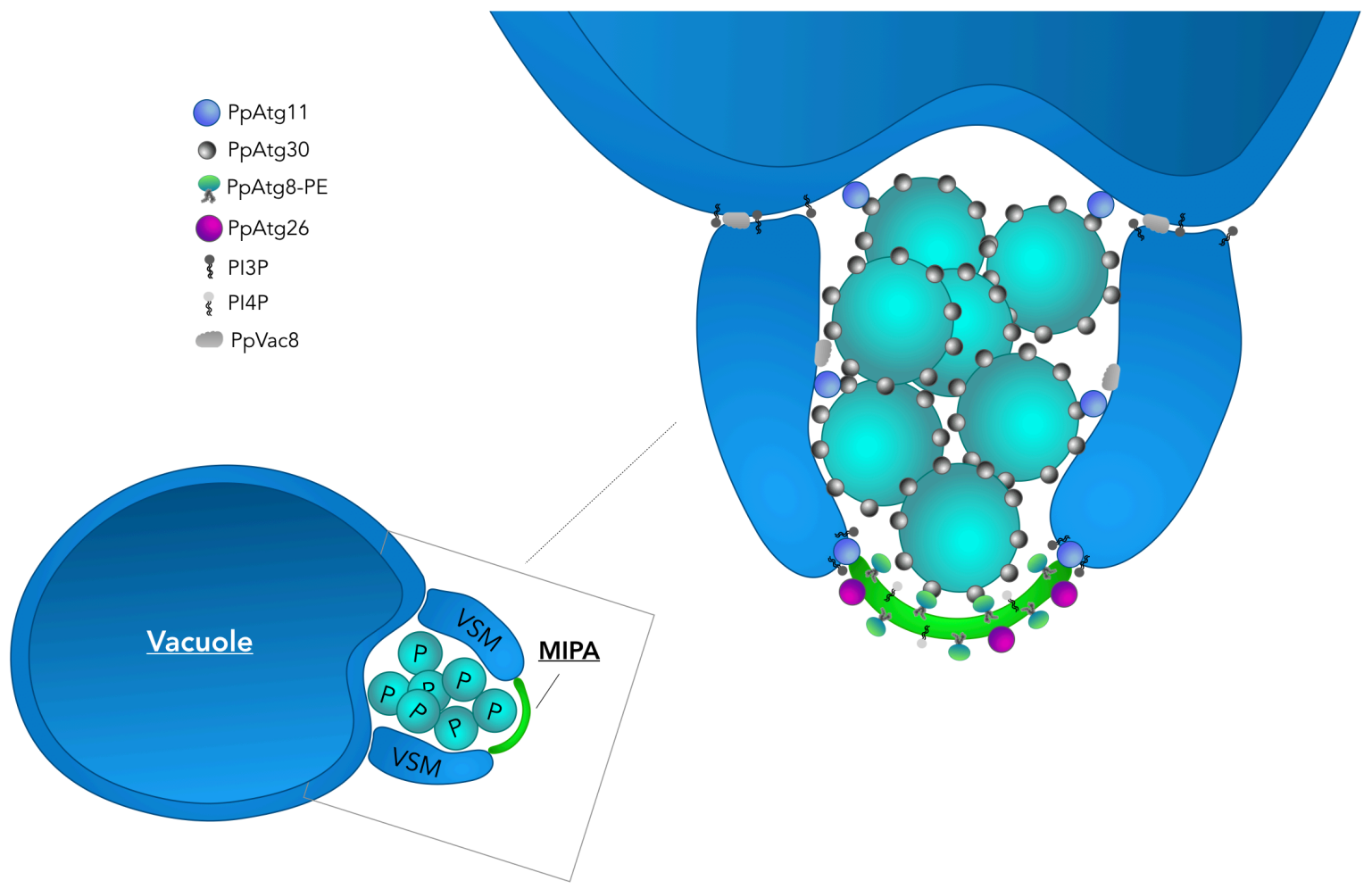

Figure 2.6: Micropexophagy in Pichia pastoris

Micropexophagy in P. pastoris was shown to involve formation of distinct membrane structures in the process of transferring peroxisomes (P) to the vacuolar lumen. Accumulated peroxisome clusters are engulfed in vacuolar sequestering membrane (VSM) extensions which are linked at their tips by a small membrane containing components of the autophagic machinery, the micropexophagic apparatus (MIPA). Involvement of PpVac8 and PpAtg11 in the vacuolar structures, and presence of PpAtg8 conjugated to PE, as well as PpAtg26 in the MIPA proposes a model that involves cargo receptor interaction with PpAtg11 and PpAtg8. So far, this involvement of autophagic structures was exclusively shown for micropexophagy in $P$. pastoris.

In S. cerevisiae, Atg36 also binds to Pex3, but its phosphorylation is mediated by the casein kinase Hrr25 (Tanaka et al., 2014). Similar to the system observed in P. pastoris, the serine residue in Atg36, S97, that corresponds to the pivotal 11-BR residue S112 in PpAtg30, was shown to be phosphorylated by Hrr25 in vitro (Farré et al., 2013; Tanaka et al., 2014). While knock-down of Hrr25 reduces 
phosphorylation of Atg36 and thereby diminishes interaction with Atg11, it does not have an effect on Pex3-Atg36 binding capacity in S. cerevisiae. Atg36 is not the only cargo receptor phosphorylated by Hrr25 enhancing interaction with the shared adapter Atg11. Both Atg19 and its paralogue Atg34, involved in the Cvtpathway, are a target of Hrr25 as well (Tanaka et al., 2014). The interaction of Hrr25 with Ypt1 and its general function in selective autophagy supports a larger role for the kinase in general autophagy (Mochida et al., 2014; Pfaffenwimmer et al., 2014).

\subsection{Nucleophagy}

Indispensable for cellular functionality and essential for genetic inheritance, the nucleus is a highly sensitive organelle. Responsible for maintenance and expression of the genome, the nucleus harbours DNA, mRNA, rRNA, the nucleolus, the site of ribosome biogenesis and different nuclear bodies. The nucleus is enveloped by the inner- (INM) and outer nuclear membrane (ONM). Metazoans maintain an additional mesh-like protein layer that provides structural stability, the nuclear lamina.

The relationship between autophagy and the nucleus has not been extensively studied, yet multiple components of the autophagic machinery have been linked to the organelle. Nuclear localisation was shown for Atg8 and LC3, and relocalisation to the nucleus was shown to be a consequence of LC3 acetylation (Klionsky et al., 2008; Ciechomska \& Tolkovsky, 2007; Kuma et al., 2007; Köchl et al., 2006; Zhang et al., 2014; Huang \& Liu, 2015).

Both macro- and microautophagic modes for degradation of nuclear material have been described: Macronucleophagy, represented by Atg39-mediated nucleophagy and micronucleophagy, represented by piecemeal microautophagy of the nucleus (PMN) (Mochida et al., 2015; Roberts et al., 2003). 
Nucleophagy has been shown to exclude DNA from degradation. In detail, exclusion of rDNA was shown to be controlled by two complexes, the chromosome linkage INM proteins (CLIP) composed of Heh1 and Nur1 and cohibin composed of Lrs4 and Csm1. The CLIP-cohibin system allows for exclusion of rDNA upon nucleolar degradation by tethering it to the INM. Cells lacking either CLIP or cohibin subunits, do not show segregation of rDNA and nucleolar proteins, but do not impair micronucleophagy either (Mostofa et al., 2018).

Another overarching characteristic among modes of nucleophagy is their initiation by TORC1 inactivation. The heterodimeric Nem1/Spo7 phosphatidic acid phosphohydrolase axis is subsequently activated, prompted by nutrient deprivation, nitrogen depletion, or rapamycin treatment (Rahman et al., 2018).

The emergence of Atg39-mediated macronucleophagy has opened up new interpretations of the data collected for micronucleophagy. Macronucleophagic degradation was not factored in and the data was therefore implied to be more representative of overall nucleophagy than micronucleophagy exclusively (Mostofa et al., 2018). Conclusive distinction between the modes of nucleophagy has become an essential prerequisite for further understanding and describing the different nucleophagic processes.

\subsubsection{Macronucleophagy}

Macronucleophagy, also termed Atg39-dependent nucleophagy (Figure 2.7), was discovered upon identification of two ER-related autophagy receptors, Atg39 and Atg40. Both receptors contain putative transmembrane domains and interact with Atg8 in an AIM-dependent manner. In addition, both receptors were shown to 
bind Atg11, with Atg39 containing an 11-BR, a fact supporting their designation as bona fide autophagy receptors (Mochida et al., 2015).

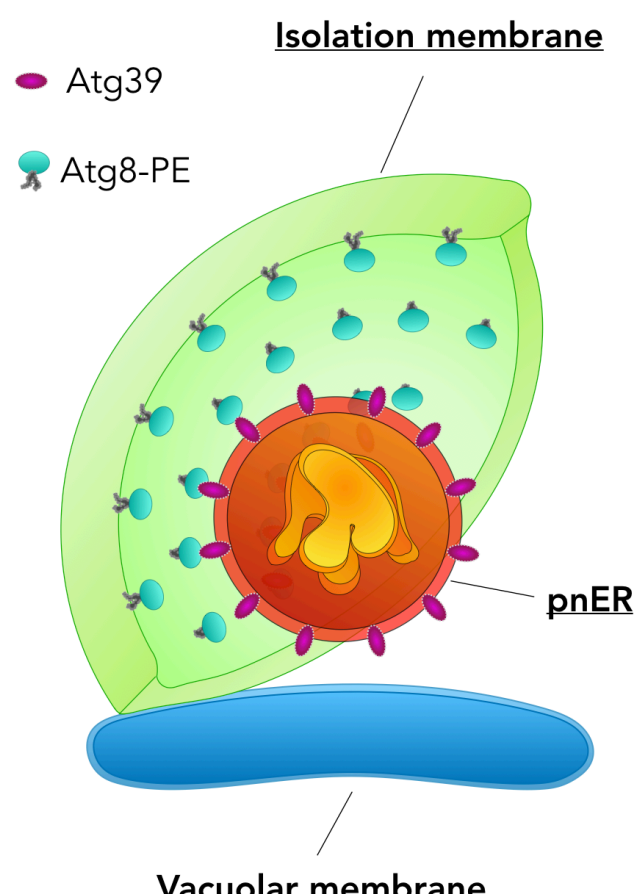

Vacuolar membrane

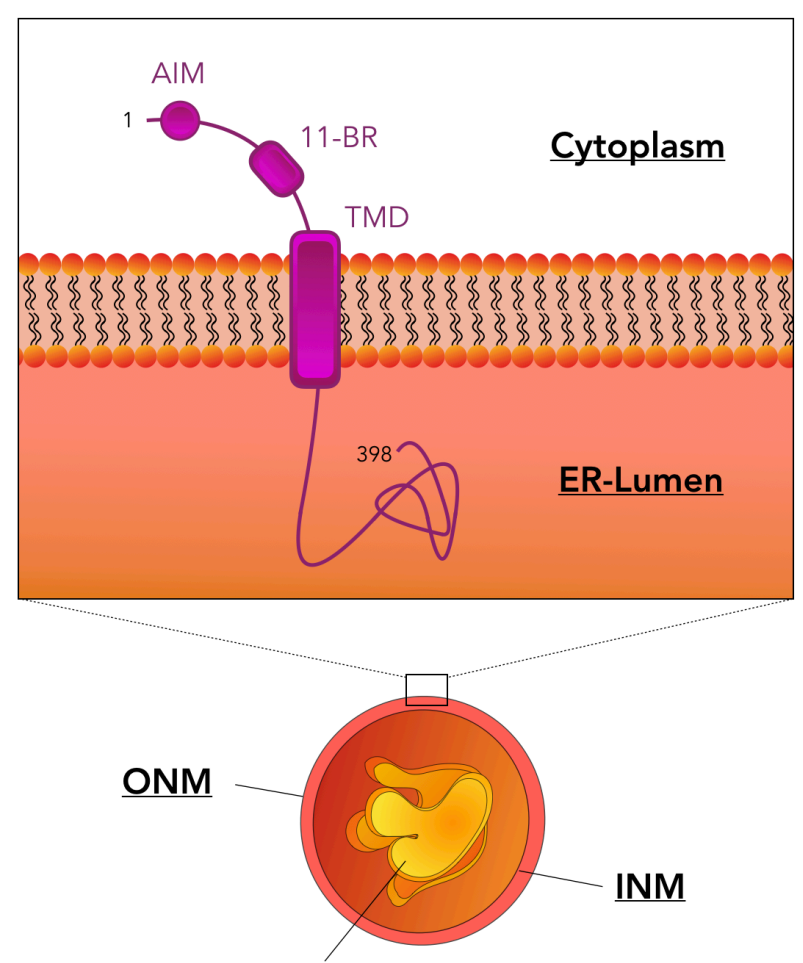

Nucleolar content

Figure 2.7: Atg39-mediated macronucleophagy

Macronucleophagy and thereby autophagy of the pnER was shown to depend on the Atg8-interacting cargo receptor Atg39. Atg39 binds Atg8 via its functional, cytosolically located AIM. Atg39 also binds the cargo adaptor Atg11 involved in selective autophagy, with a cytosolic Atg11-binding region (11-BR), substantiating its role as a bona fide cargo receptor. Atg39 contains a transmembrane domain (TMD) and is integral to the outer nuclear membrane (ONM). A large portion of Atg39 is located in the ER-lumen. In addition to double-ringed pnER structures, presumably derived from ONM and inner nuclear membrane (INM), macronucleophagic cargo was shown to contain nucleolar material as well.

Whereas either receptor is dispensable for various versions of selective autophagy including the Cvt-pathway, mitophagy and pexophagy as well as nonselective autophagy, overexpression of the receptors induces ER-phagy while deletion results in blockage of the pathway (Mochida et al., 2015). Dissection of the ER into perinuclear ER (pnER) surrounding the nucleus and cytosolic ER (cER) 
shows selective targeting of the receptors for each subtype of the ER. With Atg40 targeting cER selectively, Atg39 is the main receptor for pnER. ER-cargoes of Atg39 and Atg40 are visually distinct. Atg40 positive, cER containing autophagic bodies, show folded tubular sheets and those positive for Atg39 contain pnER and show double-ringed structures (Mochida et al., 2015).

Atg39 is an integral pnER membrane protein, with its amino terminus $(p l=4)$ facing the cytosol and its $\mathrm{C}$-terminus $(\mathrm{pl}=11)$ residing in the ER-lumen (Mochida et al., 2015; Käll et al., 2004). Cargo for Atg39-dependent nucleophagy includes the INM as well as the ONM. A portion of the nucleolus, represented by Nop1, a histone glutamine methyltransferase and component of the small subunit processome complex, was shown to be degraded as well. A ribosome biogenesis (Ribi) protein and fibrillarin homologue, the nucleolar constituent Nop1 is a substrate to both micro- and macronucleophagy (Dawaliby \& Mayer, 2010; Mochida et al., 2015). Upon overexpression, Atg39 localises to ER-vacuole contact sites. Its deletion or a disruption of either its AIM or 11-BR, results in phenotypic abnormalities observed in both nucleus and pnER and entails a decrease in cell viability (Mochida et al., 2015; Vevea et al., 2015). Cellular protein levels of Atg39 increase during lipid stress, ER-stress and nitrogen starvation as well as treatment with rapamycin (Vevea et al., 2015; Mochida et al., 2015).

\subsubsection{Piecemeal microautophagy of the nucleus}

PMN occurs at the nucleus vacuole junction (NVJ), leading to degradation of the junction itself, which includes parts of both INM and ONM, as well as vacuolar membrane and a portion of nucleoplasm (Roberts et al., 2003). Five distinct stages of PMN were described (Figure 2.8): Formation of the NVJ, a prerequisite of PMN and its first stage. Induction by nitrogen starvation initiates the second stage, resulting in bulging of the nucleus being into the vacuole, followed by the 
third stage, that results in fission of a portion of the nucleus (formation of a micronucleus). The fourth stage involves fusion of the vacuolar arms and abstraction of the PMN vesicle into the vacuolar lumen, which is finally degraded by the resident hydrolases in step five (Krick et al., 2009).
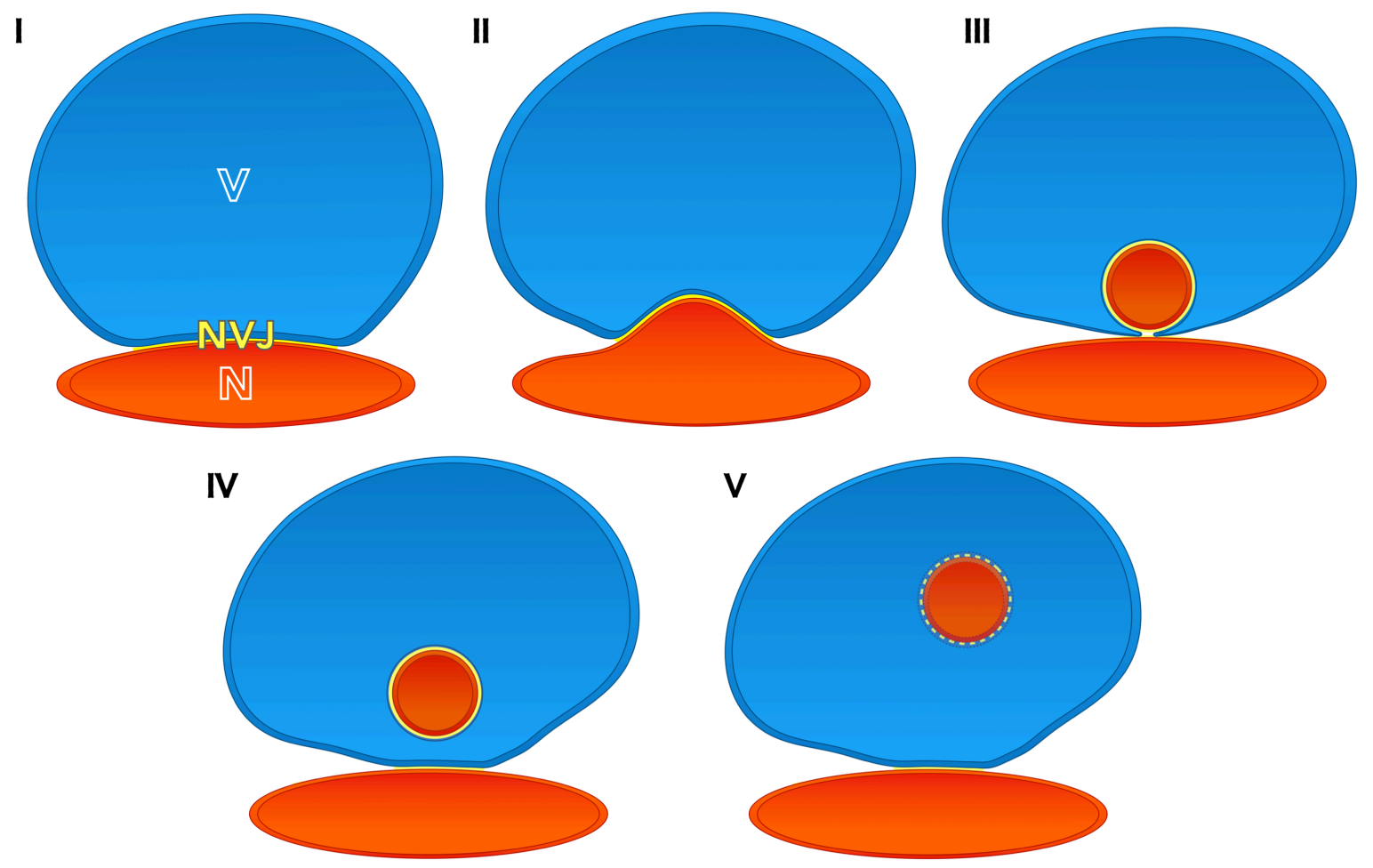

\section{Figure 2.8: Piecemeal microautophagy of the nucleus}

Piecemeal microautophagy of the nucleus (PMN) results in the abstraction of nuclear material, nucleus vacuole junction (NVJ) and vacuolar membrane into the vacuolar lumen. PMN was shown to occur by five successive stages. (I) Presence of the NVJ is a prerequisite for PMN. The PMN process is initiated by (II) bulging of the nucleus into the vacuole succeeded by (III) fission of a portion of the nucleus into a micronucleus. (IV) Subsequent fusion of vacuolar sequestering arms and thereby abstraction of a PMN vesicle into the vacuolar lumen is followed by $(\mathbf{V})$ degradation within the vacuole by resident hydrolases.

In spite of its microautophagic mechanism, PMN relies on a majority of the core Atg proteins, essential to macroautophagy, with the addition of a few subtype specific Atg-family members. PMN is suggested to be a selective autophagic type 
that requires both cargo receptor adaptor Atg11, as well as non-selective component Atg17 (Krick et al., 2008).

In addition to the core autophagic machinery, constituents of the fusion machinery, Trs85 of the TRAPPIII complex and Ypt7, are required for PMN functionality. Proteins specifically involved in the Cvt-pathway are dispensable for PMN. While cargo receptor Atg19 does not show involvement, deletion of the PIbinding Atg18 paralogue Atg21 affects PMN slightly (Krick et al., 2008). Mechanistically distinctive, PMN occurs independent of factors required for microautophagic tube formation, involving the Vtc protein complex (Krick et al., 2008; Uttenweiler et al., 2007; Krick et al., 2009). Its unusual role might be conditioned by its restriction to the NVJ or involving an extended role for the autophagic machinery.

\subsubsection{The nucleus vacuole junction}

Representative of both cargo and a crucial component involved in the PMN process, NVJs are formed by the velcro-like interaction of Nvj1, resident to the junction portion of the NE and Vac8, located in the vacuolar membrane (Roberts et al., 2003; Kvam \& Goldfarb, 2007).

The NVJ shows a highly distinct membrane organisation (Figure 2.9), excluding nuclear pore complexes (NPCs), suspending them from both the NVJ and degradation by PMN (Severs et al., 1976, Pan et al., 2000). On the vacuolar side, the ATPase subunit Vph1 was suggested to be excluded from the NVJ, a characteristic that was previously shown to coincide with the liquid ordered $\left(L_{0}\right)$ phase of the vacuolar membrane (Dawaliby \& Mayer, 2010; Toulmay \& Prinz, 2013). Sterol presence is critical for $L_{0}$ phase formation and sphingolipids show a preference for partitioning into $L_{o}$ domains implying a possible enrichment at the NVJ (Sankaram \& Thompson, 2001; Wang \& Silvius, 2003). 
In addition to its essential interaction with Vac8, Nvj1 recruits Tsc13 and Osh1, both involved in lipid metabolism, to the NVJ (Kvam et al., 2005; Kvam \& Goldfarb, 2004).

Several additional proteins localise to the NVJ independent of Nvj1. Mdm1, an ER-vacuole tether, capable of generating nucleus vacuole contact sites in absence of Nvj1 and a soluble paralogue, Nvj3, additionally localise to the NVJ (Hariri et al., 2017).

\subsection{Nvj1 dependent components}

Nvj1 is a transmembrane protein of the NVJ, with its C-terminal domain extending into the NVJ and linking INM and ONM by its transmembrane domain and its INM anchor, respectively. ONM and INM are clamped by Nvj1, resulting in reduction of the perinuclear space from $18 \mathrm{~nm}$ present in the NE to $9 \mathrm{~nm}$ within the NVJ (Millen et al., 2008). Loss of Nvj1 is accompanied by a decrease in cell survival upon nitrogen starvation. Deletion of Vac8 results in a loss of the Nvj1 focal pattern, it is then instead found dispersed throughout the pnER (Mostofa et al., 2018; Pan et al., 2000).

Vac8 is involved in a variety of processes, present in distinct domains of the vacuolar membrane. A multivalent adapter, containing at least eight armadillo repeat (ARM) domains, Vac8 provides distinct interaction sites for various partners (Tang et al., 2006; Tewari et al., 2010). A cationic triad (R276, R317, R359) within the ARM domains of Vac8 competitively and exclusively binds either Nvj1 or Atg13. The interaction of $\operatorname{Vac} 8$ with Atg13 was shown to be required for functionality of the Cvt-pathway (Scott et al., 2000; Jeong et al., 2017).

Vac8 shows localisation to the NVJ and accumulation at the interface of vacuoles in close proximity to sites enriched in ergosterol, potentially facilitated by its 
palmitoylated state (Fratti et al., 2004; Levental et al., 2010; Smotrys \& Linder, 2004).

Multiple posttranslational modifications for Vac8 were described: Phosphorylation, myristoylation and palmitoylation. While effects of phosphorylation have not yet been understood, both myristoylation and palmitoylation occur at the $\mathrm{N}$-terminal Src homology 4 (SH4) domain and enable anchoring of Vac8 to the vacuolar membrane and its targeting towards subcompartments within (Scott et al., 2000; Wang et al., 1998; Pan et al., 2000; Peng et al., 2006). Palmitoylation is facilitated by three consecutive cysteine residues, recognised by the protein acyltransferase Pfa3, a process that is most efficient for previously myristoylated Vac8 (Hou et al., 2005; Smotrys \& Linder, 2004; Nadolski \& Linder, 2009). Since thioester linkage is the underlying basis for palmitoylation, it represents the only known reversible form of protein lipidation (Dietrich \& Ungermann, 2004). Consistent with this mechanism, Vac8 abolishes localisation to the vacuolar membrane upon disruption of palmitoylation, an effect that impairs vacuole inheritance, yet sustains Cvt-pathway functionality (Wang et al., 1998).

Essential for vacuolar inheritance, Vac8 binds Vac17. As an adaptor to Myo2, the yeast class five myosin anchors actin filaments to vacuoles and enables directed movement towards the bud (Hou et al., 2005; Tang et al., 2003; Wang et al., 1998). Vac8 is also involved in homotypic vacuole fusion (Veit et al., 2001; Wang, 2001).

Apart from the essential components Nvj1 and Vac8, Osh1 is another resident protein of the NVJ and a member of the yeast Osh family that bear both structural and functional homology to oxysterol-binding proteins (OSBPs) of the mammalian system (Levine \& Munro, 2001). OSBPs and OSBP-related proteins (ORPs) have been shown to specifically bind oxysterols, oxygenated derivatives of 
cholesterol (ergosterol in yeast), with yeast containing 7 ORP genes and humans 12 (Lehto et al., 2001). ORPs have been proposed to facilitate transport sterols and phospholipids between intracellular membranes (Mesmin \& Antonny, 2016). ORPs in humans and yeast contain a variety of domains such as pleckstrin homology $(\mathrm{PH})$ domain, the FFAT domain containing a EFFDAxE sequence with two phenylalanines and an acidic tract, a Golgi dynamics (GOLD) domain, ankyrin repeats (ANKs) and the core OSBP-related domain (ORD) (Tong et al., 2016). PH, FFAT and ANK domains convey targeting ability to membrane lipids and intramembrane proteins (Raychaudhuri \& Prinz, 2010). The C-terminally located ORDs contain the OSBP fingerprinting motif EQVSHHPP and conserved basic residues that enable recognition of the phosphatidylinositol 4-phosphate (PI4P) head group. Binding of PI4P is a shared trait amongst ORPs and an essential aspect for ORP functionality (Tong et al., 2013).

Utilizing PI4P-exchange as a driving force for exchange with a secondary lipid between organellar membranes against concentration gradients was shown for sterols in Osh4 and for phosphatidylserine in Osh6, Osh7, ORP7 and ORP8 (Chung et al., 2015; de Saint-Jean et al., 2011; Mesmin et al., 2013; Moser von Filseck et al., 2015). The secondary ligand of the Osh1 ORD is ergosterol, allowing counter-transport with PI4P (Manik et al., 2017). A general feature of the $\mathrm{PH}$ domain is its capacity to bind PI4P, yet specific interactions are present as well. The $\mathrm{PH}$ domain of Osh1 enables targeting to late Golgi, interacting with phosphatidylinositol (PI) (Levine \& Munro, 2001). Osh1 is recruited from Golgipools within the cytoplasm to the NVJ via its ANK domain in an Nvj1-dependent manner, recognising its small cytosolic segment (Levine \& Munro, 2001; Kvam \& Goldfarb, 2004; Manik et al., 2017). Results for Osh1 were shown to support the bridge and shuttle model proposed for ORPs (Figure 2.9). The N-terminal ORD interacts with both membranes of the organellar contact site, enabled by long flexible loops. 


\section{Va๕นolar Tumen}

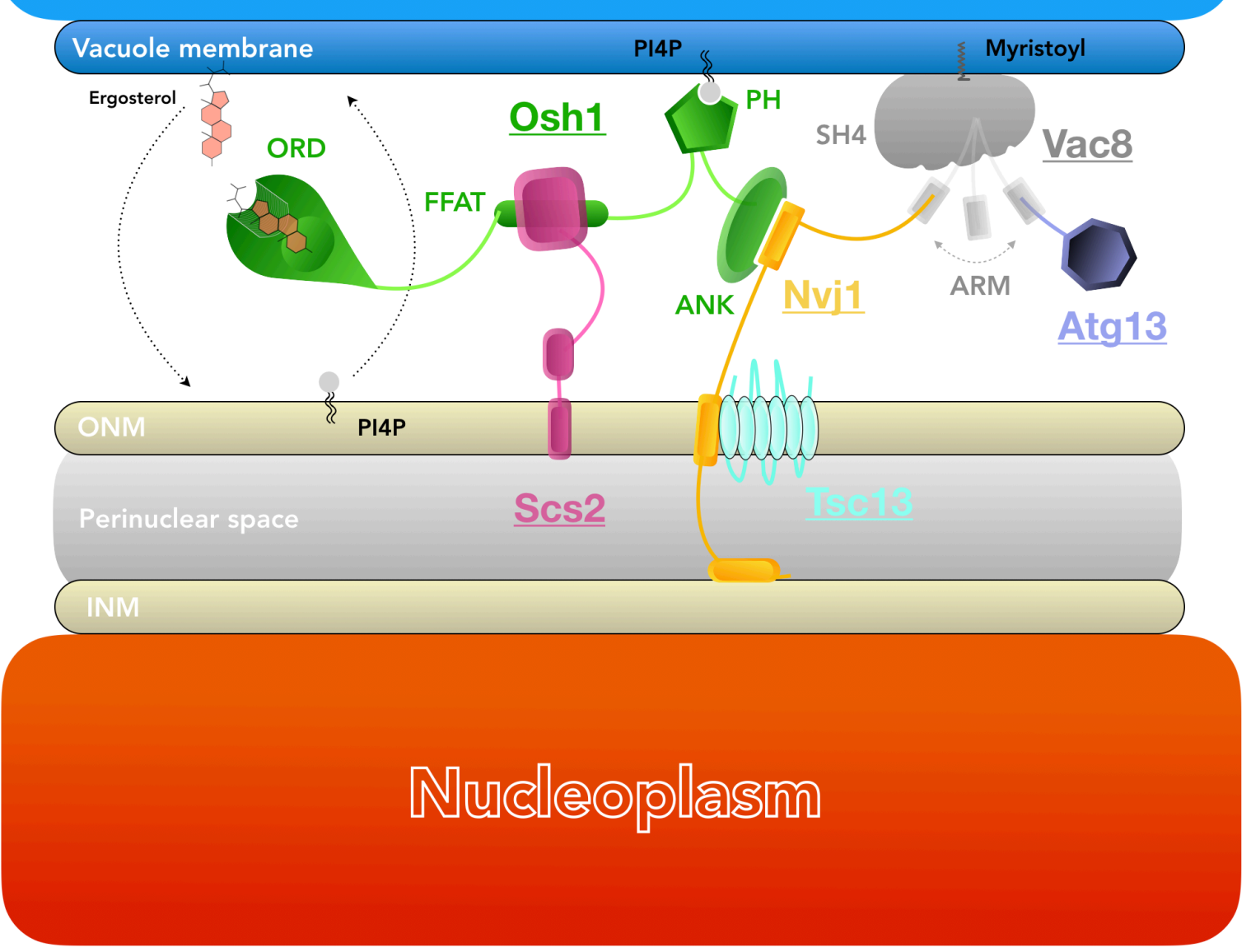

Figure 2.9: Constituents of the nucleus vacuole junction

Components of the NVJ, that depend on recruitment by the core component Nvj1. The competitive interaction of $\mathrm{Vac} 8$ with both $\mathrm{Nvj} 1$ and Atg13 is depicted. Both Nvj1 and Atg13 bind to the same catalytic amino acid triad within the armadillo repeat (ARM) domain of Vac8, which is in turn myristoylated at its Src homology 4 (SH4) domain and is thereby anchored to the vacuolar membrane. Nvj1 clamps inner and outer nuclear membrane (INM and ONM) and recruits Osh1 by interaction with its ankyrin repeat (ANK) domain. Tsc13 is also recruited to the NVJ in an Nvj1 dependent manner. The pleckstrin homology (PH) domain of Osh1 interacts with phosphoinositol-4-phosphate (PI4P) and its FFAT motif interacts with the endoplasmatic reticulum (ER) resident protein Scs2. The Osh1 oxysterol binding protein related domain (ORD) is thought to function as an exchanger of PI4P and sterol from one membrane to the opposing membrane. 
Osh1 was shown to be recruited to the NVJ in direct proportion to cellular levels of Nvj1 and is a substrate of PMN. While Osh1 is not required for formation of NVJs and PMN in starvation conditions, deletion of all Osh family proteins results in inhibition of PMN activity (Kvam \& Goldfarb, 2004). While Osh1 binds Nvj1 via its ANK repeats, it also binds the ER-protein Scs2, a vesicle-associated membrane protein (VAMP)- associated ER protein (VAP) homologue, via its FFAT motif. Alongside its paralogue Scs22, Scs2 is required for formation of membrane contact sites (MCSs) between ER and PM (Manford et al., 2012). Interaction of Osh1 with Scs2 occurs at the NVJ, with additional interactions observed at potential Golgi. Scs2 is described to be the predominant anchor of Osh proteins to the ER and was shown to enhance targeting of Osh1 to the NVJ (Loewen et al., 2003). Deletion of Osh1 results in emergence of phenotypic effects, akin to those observed in erg $\Delta$ strains, defective in ergosterol synthesis (Jiang et al., 1994; Levine \& Munro, 2001). Additionally, deletion of Erg5 results in significant reduction of PMN activity (Dawaliby \& Mayer, 2010).

Another substrate of PMN, the essential enoyl-CoA reductase Tsc13, accumulates at NVJs in a Nvj1-dependent manner. Tsc13 was proposed to catalyse the last step in each cycle of very long chain fatty acid (VLCFA) elongation (Kohlwein et al., 2001; Gable et al., 2004; Kvam et al., 2005). A mutation of Tsc13 (Q81K) that results in $\sim 50 \%$ reduction of fatty acid elongation activity in vitro (Kohlwein et al., 2001) was shown to also result in a significant decrease of lumenal diameter in PMN blebs. The same effect was observed upon incubation of cells with cerulenin, a compound inhibiting de novo fatty acid synthesis and elongation (Kvam et al., 2005).

Nvj2 is a resident protein of the NVJ that depends on both Nvj1 and Vac8 for its localisation, but is dispensable for NVJ formation (Toulmay \& Prinz, 2012). Nvj2 contains a putative $\mathrm{PH}$ domain and a synaptotagmin-like-mitochondrial-lipid 
binding protein (SMP) domain, a highly conserved feature, that is found in only six other proteins in yeast (Toulmay \& Prinz, 2012). The SMP domain belongs to the tubular lipid-binding (TULIP) superfamily, defined by presence of a hydrophobic cavity which enables lipid binding. While its exact function remains elusive, all seven proteins containing the SMP domain localise to MCS, with a part of them showing involvement in tethering of the corresponding membranes (Kopec et al., 2010; Lee, 2006).

\subsection{Nvj1 independent nucleus vacuole contact sites}

Idependent of Nvj1 various proteins were shown to localise to the NVJ (Figure 2.10). The NVJ is additionally tethered by Mdm1, which interacts with fatty acylCoA synthases and clusters LDs in close proximity of the NVJ periphery. Highly conserved in mammals, represented by four SNXs (Snx13, Snx14, Snx19 and Snx25), Mdm1 is an ER-vacuole tether that localises specifically to the pnER (Thomas et al., 2014; Henne et al., 2015). The transmembrane protein Mdm1 is anchored to the ER and binds PI3P in the vacuolar membrane via a C-terminally located phox homology (PX) domain. Mdm1 localises to the NVJ in an Nvj1independent fashion and its overexpression results in hypertethering of the pnER to the vacuole. Mdm1 was also shown to be a substrate of PMN and yet is dispensable for PMN functionality (Hariri et al., 2018; Henne et al., 2015).

A paralogue of Mdm1, Nvj3 was characterised as a soluble version of Mdm1 and the only other PX-associated protein in yeast. Like Mdm1, it is recruited independent from Nvj1 to the NVJ, but depends on Mdm1 for its NVJ localisation.

The MCS monomeric protein Lam6/Ltc1 was shown to localise to both NVJ and non-NVJ ER-vacuole contact sites, including the ERMES. Lam6/Ltc1 localises to the ER-vacuole contact sites in Vac8-dependent but Nvj1-independent fashion. Its 
is restricted to ER-mitochondria contact sites upon deletion of Vac8, yet retains localisation to ER-vacuole contacts upon deletion of Nvj1 (Murley et al., 2015). Lam6/Ltc1 contains a glucosyltransferase, Rab-like GTPase activators and myotubularins (GRAM) domain similar in structure to PH domains (Begley et al., 2003). The GRAM domain is necessary for lipid independent ERMES localisation, requiring Tom70/71, but not Vac8 (Tong et al., 2018). A Vad1 analog of StARrelated lipid transfer (VASt) domain is predicted to form a hydrophobic pocket capable of harbouring lipid molecules in Lam6/Ltc1 and a hydrophobic $\alpha$-helix is located at its C-terminus while its $\mathrm{N}$-terminus is unstructured (Murley et al., 2015; Khafif et al., 2014). Capable of cholesterol transfer between membranes in vitro, Lam6/Ltc1 is suggested to be a sensor and/or transporter of sterols in vivo (Murley et al., 2015). Proposed for promoting formation of specialised membrane domains to regulate localisation of proteins involved in membrane biogenesis and signalling, Lam6/Ltc1 is also suggested to interact with a subunit of TORC1, Kog1 and its downstream target Npr1 hinting at a role for TORC1 regulation upon domain formation (Murley et al., 2015). The Rag GTPase component Gtr2 of the exit from $G_{0}(E G O)$ complex, a highly conserved upstream regulator of TORC1 (Dubouloz et al., 2005; Bonfils et al., 2012), is localised in ergosterol enriched vacuolar domains alongside Lam6/Ltc1 (Toulmay \& Prinz, 2013).

A large, highly conserved $358 \mathrm{kDa}$ protein, Vps13 was shown to be located at multiple sites including the NVJ. It was previously observed at endosomes, the prospore membrane and the vacuolar and mitochondrial patch (vCLAMP) (Huh et al., 2003; Park et al., 2013, 2016; Lang et al., 2015). Vps13 is implicated in vesicle fusion, actin regulation and autophagy, and its localisation was shown to be dependent on nutrient availability (Brickner \& Fuller, 1997; Föller et al., 2012; Muñoz-Braceras et al., 2015). Vps13 is present at endosomes and endosomemitochondria contact sites during mitotic growth in presence of high glucose levels, but changes to NVJs upon glucose limitation (Lang et al., 2015; Park et al., 
2016). Vps13 is able to bind phosphatidic acid and Pls in vitro, it contains an Nterminal chorein repeat domain, an APT1 domain, two C-terminal Atg2 domains, a putative $\mathrm{PH}$ domain and a central Vps13 adaptor binding (VAB) domain (Velayos-Baeza et al., 2004; Fidler et al., 2016; Rzepnikowska et al., 2017; Koizumi \& Gallagher, 2013).

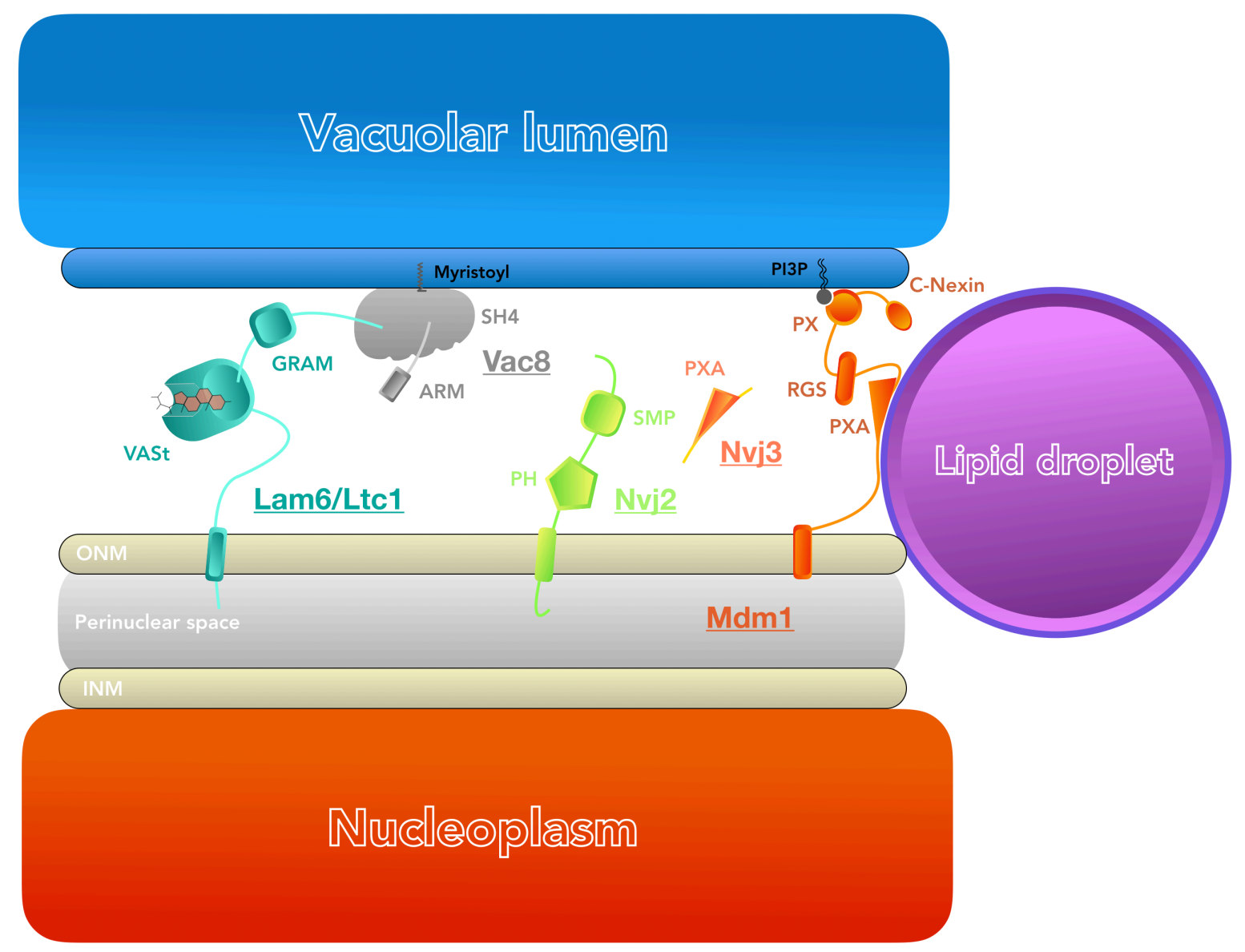

Figure 2.10: Additional components of nucleus vacuole contact sites

Multiple proteins are localised to the NVJ but recruited independently of Nvj1. Lam6/ Ltc1 binds to Vac8 in the vacuolar membrane and is anchored to the ER with its $\mathrm{N}$ terminus. Lam6/Ltc1 contains a glucosyltransferases, Rab-like GTPase activators and myotubularins (GRAM) domain and a Vad1 analog of StAR-related lipid transfer (VASt) domain, that is predicted to convey sterol transport ability to Lam6/Ltc1. Lam6/Ltc1 is found in the NVJ but also in non-NVJ ER-vacuole contacts. Mdm1 was shown to bind lipid droplets at the rim of the NVJ via its PXA and N-terminal domain. Mdm1 is able to form ER vacuole contacts independent of the NVJ. Nvj3, a soluble paralogue of Mdm1 and also found at ER-vacuole contacts, was shown to contain a PXA domain as well. Nvj2 was determined to localise to the NVJ, but is not required for NVJ formation. It contains a synaptotagmin-like-mitochondrial-lipid binding protein (SMP) domain, and a putative $\mathrm{PH}$ domain. 
The VAB domain was identified acting as an adaptor for the SNX Ypt35 that enables binding of Vps13 to endosomes and the vacuolar membrane via a unique $\Phi_{x \times} \Phi_{\times} P_{x} \Phi_{\times} \Phi$ ( $\Phi$ represents hydrophobic amino acids) consensus containing binding motif (PxP), also found in meiosis adaptor Spo71 and mitochondrial membrane protein Mcp1. All three adaptor proteins compete for Vps13 binding via its VAB domain (Bean et al., 2018). The C-terminus of Ypt35 contains a PX domain presumably required for its membrane targeting (Vollert \& Uetz, 2004). Like Vps13, Ypt35 accumulates at NVJs and localisation of Vps13 is strongly reduced upon deletion of Ypt35 and vice versa. Therefore, Ypt35 appears to be responsible for recruitment of Vps13 to the vacuole, while redistribution to the NVJ is dependent on another factor (Bean et al., 2018).

\subsection{Medical relevance of nucleophagy}

Involvement of autophagy in many diseases has made the topic a highly relevant system for formulation of novel treatment. Nucleophagy in particular, has emerged as an autophagic system heavily involved in various human pathologies (Fu et al., 2018). Autophagic degradation of the nucleus occurs in healthy cells and was shown to be required for cellular maintenance, while its impairment leads to abnormalities in nuclear shape, disruption of the NE and reduced cellular viability (Park et al., 2009; Papandreou \& Tavernarakis, 2019).

Perturbation of nucleophagy causes parakeratosis, the retention of nuclei in the stratum corneum of the epidermis and has been implicated in psoriasis (Akinduro et al., 2016). Autophagy was shown to degrade the nuclear lamina constituent Lamin B, by interacting with LC3, a mechanism observed in tumour suppression. Disruption of the process results in promotion of oncogenesis and a decrease in cellular senescence (Dou et al., 2015). 
Heightened formation of micronuclei (small portions of nuclear material) has been observed in multiple cancer types, Bloom syndrome and neurodegenerative diseases such as Alzheimer's and Parkinson's disease, while degradation was shown to be carried out by the autophagic machinery (Luzhna et al., 2013; Yankiwski et al., 2000; Petrozzi et al., 2002; Rello-Varona et al., 2012).

Extrusion of chromatin fragments from the nucleus to the cytoplasm have shown enrichment of the DNA damage marker $\mathrm{YH} 2 \mathrm{AX}$. The mechanism is presumed to be dedicated to removal of damaged DNA fragments from the nucleus. Involvement of the autophagic machinery was determined by colocalisation of the chromatin fragments with LC3, the cargo receptor p62/SQSTM1 and the lysosome (Ivanov et al., 2013).

Induction of DNA damage by exposure to etoposide was shown to increase both autophagic activity and expression of the human Atg6 orthologue Beclin 1 and cancer cells deficient in autophagy were shown to accumulate damaged DNA (Shimizu et al., 2012; Karantza-Wadsworth et al., 2007). Allelic loss of Beclin 1, is one of the most frequent genetic alterations found in human breast and ovarian cancer, an effect associated with an increase in cancer aggression, that is independent of BRCA1 loss (Liang et al., 1999; Valente et al., 2014). Beclin 1 is a tumour suppressor in mice and allelic loss was shown to promote mammary tumourigenesis, DNA damage and genomic instability (Cicchini et al., 2014; Qu et al., 2003; Yue et al., 2003; Karantza-Wadsworth et al., 2007).

Aggregation of polyglutamine (polyQ)-proteins was shown in case of huntingtin to disrupt interaction between Ataxin 3 and Beclin 1 leading to an increase in its proteasomal degradation and as a consequence, reduced autophagy (Ashkenazi et al., 2017). Cellular pathology of the polyQ disease dentatorubral-pallidoluysian atrophy is characterised by nuclear degeneration through nucleophagy-based 
Lamin B1 degradation, evidenced by accumulation of p62 and simultaneous downregulation of LC3 conjugation (Baron et al., 2017).

Beyond direct effects of disruption in nucleophagy, a number of NVJ components were shown to be associated with human disease.

The four SNX, Snx13, Snx14, Snx19 and Snx25 corresponding to NVJ resident protein $\mathrm{Mdm} 1$ in yeast are connected to autosomal recessive neurological diseases that result in granular plaque accumulation in late endosomes and autophagosomes as well as cellular vacuolisation. Introduction of diseaseanalogous Snx14-mutations into Mdm1 leads to impairment of ER-vacuole tethering and induces hypersensitivity to sphingolipid synthesis inhibitor myriocin (Thomas et al., 2014; Henne et al., 2015; Bryant et al., 2018; Datta et al., 2019).

A mutation (P56S) in Scs2 homologue VAP-A was linked to familial amyotrophic lateral sclerosis in humans exercising aggregation of the protein and resulting in ER stress and cytotoxicity (Teuling et al., 2007; Prosser et al., 2008). Scs2 interactor Osh1 is highly conserved in mammals, as are the OSBPs. Their upregulation is correlated with high blood pressure and hypertension and observed in cases of morbid obesity (Ngo \& Ridgway, 2009; Bouchard et al., 2009).

NVJ resident protein Vps13 is represented by four functionally distinct VPS13 proteins in humans (VPS13A-D), that have not been located at MCS but their disruption results in development of various diseases, namely Choreaacanthocytosis, Cohen syndrome, Parkinson's disease, and spastic ataxia, respectively (Rampoldi et al., 2001; Kolehmainen et al., 2003; Lesage et al., 2016; Seong et al., 2018). 


\subsection{Aim of the study}

Macroautophagy, often simply referred to as autophagy, remains the central focus of investigations into the autophagic system. Microautophagy, is in comparison poorly understood. Autophagy of the nucleus, termed nucleophagy, has emerged as a highly relevant topic in the autophagic and medical fields and was shown to be realised in both macro- and micronucleophagic fashion.

Differentiation of macro- and micronucleophagy has presented to be difficult so far, resulting in ambiguity concerning results obtained for the different modes of nucleophagy. Establishing a reliable system allowing for distinction of the nucleophagic modes is an essential prerequisite for further understanding purpose and mechanism of the nucleophagic systems.

A representative mode of microautophagy, PMN was shown to require the core autophagic machinery, but its molecular mechanisms, cargo specificity and selectivity have yet to be resolved. Characterisation of components, designated purpose and selectiveness are further explored in the present study. 


\section{Materials and methods}

\subsection{Materials}

\subsubsection{Software and databases}

Table 3.1: Software and databases used in this study

\begin{tabular}{|c|c|}
\hline Software/database & Reference \\
\hline Adobe ${ }^{\circledR}$ Creative Suite ${ }^{\circledR} 6$ & Adobe Systems, San Jose, California, USA \\
\hline EndNote X7 & Clarivate Analytics, Philadelphia, Pennsylvania, USA \\
\hline Fiji & Schindelin et al. (2012) \\
\hline Huygens Professional 18.10.0 & Scientific Volume Imaging, Hilversum, Netherlands \\
\hline iLIR 1.0 & Kalvari et al. (2014) \\
\hline ImageJ2 & Rueden et al. (2017) \\
\hline Imspector 0.14 & Abberior Instruments GmbH, Göttingen, Germany \\
\hline Pages 7.2 & Apple Inc., Cupertino, California, USA \\
\hline Microsoft ${ }^{\circledR}$ Office for Mac & Microsoft, Redmond, Washington, USA \\
\hline \multicolumn{2}{|l|}{16.16 .2} \\
\hline Prism 7.0d for Mac OS X & GraphPad Software, San Diego, California, USA \\
\hline SnapGene ${ }^{\circledR} 4.0 .8$ & GSL Biotech, Chicago, Illinois, USA \\
\hline softWoRx ${ }^{\circledR}$ & Applied Precision, Issaquah, Washington, USA \\
\hline Keynote 8.2 & Apple Inc., Cupertino, California, USA \\
\hline
\end{tabular}




\subsubsection{Yeast and bacteria strains}

\subsubsection{S. cerevisiae strains}

Table 3.2: Saccharomyces cerevisiae strains used in this study

\begin{tabular}{|c|c|c|}
\hline Strain & Genotype & Reference \\
\hline WCG4a & MAT $\alpha$ his2-11,15 leu2-3,112 ura3 & Thumm et al. (1994) \\
\hline $\operatorname{atg} 1 \Delta$ & $\begin{array}{l}\text { WCG4a MAT } \alpha \text { his 2-11,15 leu2- 3,112 } \\
\text { ura3 atg1 } \triangle:: \text { natNT2 }\end{array}$ & This work \\
\hline$n v j 1 \Delta$ & 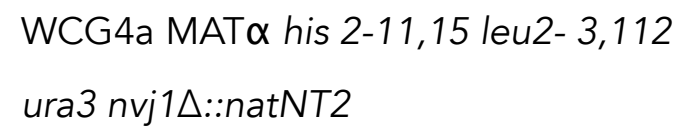 & This work \\
\hline pep4 4 & 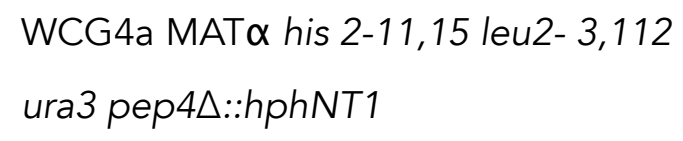 & This work \\
\hline myo1s & $\begin{array}{l}\text { MATa his3 leu2 lys2 trp1 ura3 } \\
\text { myo1 } \triangle: \text { :KanMX6 }\end{array}$ & Bi et al. (1998) \\
\hline $\operatorname{atg} 39 \Delta$ & 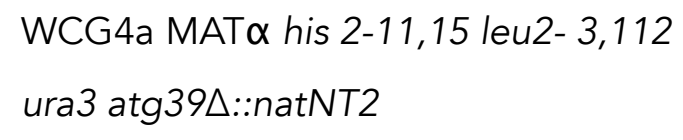 & $\begin{array}{l}\text { Petra Schlotterhose (AG } \\
\text { Thumm) }\end{array}$ \\
\hline $\operatorname{vac} 8 \Delta$ & 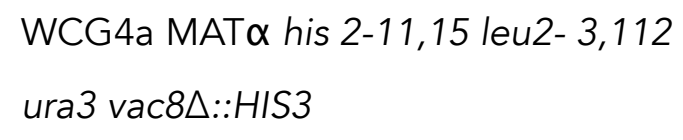 & Krick et al. (2008) \\
\hline $3 \times m$ TagBFP-Pho8 & $\begin{array}{l}\text { WCG4a MAT } \alpha \text { his2-11,15 leu2-3,112 } \\
\text { ura3 3×mTagBFP-Pho8::natNT2 }\end{array}$ & This work \\
\hline Vph1-yEGFP & $\begin{array}{l}\text { WCG4a MATa his 2-11,15 leu2- 3,112 } \\
\text { ura3 Vph1-yEGFP::hphNT1 }\end{array}$ & $\begin{array}{l}\text { Lena Munzel (AG } \\
\text { Thumm) }\end{array}$ \\
\hline $\begin{array}{l}3 \times m \text { TagBFP-Pho8 } \\
\text { Vph1-yEGFP }\end{array}$ & 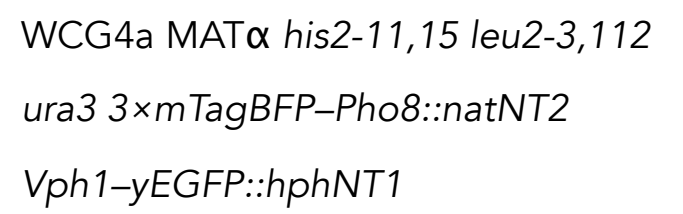 & This work \\
\hline
\end{tabular}




\subsubsection{Escherichia coli strains}

Table 3.3: Escherichia coli strains used in this study

\begin{tabular}{|c|c|c|}
\hline Strain & Genotype & Reference \\
\hline \multirow[t]{3}{*}{ XL1-Blue } & recA1 endA1 gyrA96 thi-1 hsdR17 & Agilent Technologies, Inc., \\
\hline & supE44 relA1 lac [F' proAB & Santa Clara, California, USA \\
\hline & laclqZ $\Delta M 15 \operatorname{Tn} 10$ (Tetr)] & \\
\hline \multirow[t]{4}{*}{ XL10-Gold ${ }^{\circledR}$} & Tetr $\Delta(\mathrm{mcrA}) 183 \Delta$ (mcrCB-hsdSMR- & Agilent Technologies, Inc., \\
\hline & mrr)173 endA1 supE44 thi-1 recA1 & Santa Clara, California, USA \\
\hline & gyrA96 relA1 lac Hte [F' proAB & \\
\hline & laclqZ $\Delta M 15$ Tn10(Tetr) Amy Camr] & \\
\hline \multirow[t]{3}{*}{$\mathrm{NEB}^{\circledR}$ 5-alpha } & fhuA2 $\Delta$ (argF-lacZ)U169 phoA glnV44 & New England Biolabs \\
\hline & Ф80 $\triangle$ (lacZ)M15 gyrA96 recA1 relA1 & GmbH, Frankfurt am Main, \\
\hline & endA1 thi-1 hsdR17 & Germany \\
\hline
\end{tabular}

\subsubsection{Plasmids}

\section{Table 3.4: Plasmids used in this study}

The first line marks all constructs used for plasmid expression in S. cerevisiae. The second line marks constructs used for genomic insertions by homologous recombination. All constructs required for construction of target plasmids are listed below the second line.

\begin{tabular}{|c|c|c|}
\hline Plasmid & Genotype & Reference \\
\hline Nvj1-yEGFP & $\begin{array}{l}\text { pRS316 P } \text { NVJ1 }_{1}-N_{v j 1}-y E G F P- \\
T_{C Y C 1}\end{array}$ & This work \\
\hline GFP-Atg8 & $\begin{array}{l}\text { pRS315 P } \text { ATG8 }_{\text {GFP-Atg8- }}- \\
\text { TATG8 }_{\text {AT }}\end{array}$ & $\begin{array}{l}\text { Sebastian Bremer (AG } \\
\text { Thumm) }\end{array}$ \\
\hline GFP-Atg8 & $\begin{array}{l}\text { pRS316 } \text { PATG8 }_{\text {ATFP-Atg8- }}- \\
\text { TATG8 }_{\text {AT }}\end{array}$ & Suzuki et al. (2001) \\
\hline Atg39-yEGFP & $\begin{array}{l}\text { pRS316 P } \text { ATG39-Atg39- }_{\text {AT }}- \\
\text { yEGFP-T } \\
\text { CYC1 }\end{array}$ & This work \\
\hline
\end{tabular}




\begin{tabular}{|c|c|c|}
\hline Plasmid & Genotype & Reference \\
\hline \multirow[t]{2}{*}{ yEGFP-Atg11 } & pUG36 P PET17-yEGFP- & This work \\
\hline & Atg11-TCYC1 & \\
\hline \multirow[t]{2}{*}{ GFP-FYVE $E_{E A A 1}$} & pRS316 P PRC1-GFP- & Burd \& Emr (1998) \\
\hline & $F Y V E_{E E A 1}$ & \\
\hline \multirow[t]{3}{*}{$\mathrm{NLS}_{\mathrm{Nab} 2}-\mathrm{m}$ Cherry } & $\mathrm{pRS} 413 \mathrm{P}_{\mathrm{TEF} 1}-\mathrm{NLS}_{\mathrm{Nab2}}-$ & Michael Rout (The \\
\hline & mCherry & Rockefeller University, New \\
\hline & & York, New York, USA) \\
\hline \multirow[t]{2}{*}{ 3×mTagBFP-Pho8 } & pRS305 P PGK1-3×mTagBFP_ & Graef et al. (2013) \\
\hline & Pho8 & \\
\hline pFA6a hphNT1 & Amp $p^{R}$ ori $P_{T E F 1}-H_{y g}{ }^{R-} T_{C Y C 1}$ & Janke et al. (2004) \\
\hline pFA6a natNT2 & Amp ${ }^{R}$ ori $P_{T E F 1}-N_{r s}{ }^{R}-T_{A D H 1}$ & Janke et al. (2004) \\
\hline pRS305 & Amp $p^{R}$ ori lacZ' LEU2 & Sikorski \& Hieter (1989) \\
\hline \multirow[t]{2}{*}{ pRS313 } & CEN/ARS Amp ${ }^{R}$ ori lacZ' & Sikorski \& Hieter (1989) \\
\hline & HIS3 & \\
\hline \multirow[t]{2}{*}{ pRS315 } & CEN/ARS Amp ${ }^{R}$ ori lacZ' & Sikorski \& Hieter (1989) \\
\hline & LEU2 & \\
\hline \multirow[t]{2}{*}{ pRS316 } & CEN/ARS Amp ${ }^{R}$ ori lacZ' & Sikorski \& Hieter (1989) \\
\hline & URA3 & \\
\hline \multirow[t]{2}{*}{ pRS413 } & CEN/ARS Amp ${ }^{R}$ ori lacZ' & Sikorski \& Hieter (1989) \\
\hline & HIS3 & \\
\hline \multirow[t]{2}{*}{ pUG36 } & CEN/ARS AmpR ori URA3 & Niedenthal et al. (1996) \\
\hline & $P_{\mathrm{MET} 17-y E G F P-l a c Z^{\prime}-T_{C Y C 1}}$ & \\
\hline
\end{tabular}




\subsubsection{Synthetic oligonucleotides}

Synthetic oligonucleotides were designed using SnapGene ${ }^{\circledR}$ 4.0.8 (GSL Biotech) and subsequently synthesised by Eurofins Genomics GmbH (Ebersberg, Germany).

Table 3.5: Synthetic oligonucleotides used in this study

Oligonucleotides above the line were used for amplification of genomic DNA, those below the line for generation of DNA designed for homologous recombination.

\begin{tabular}{|c|c|}
\hline Oligonucleotide name & Sequence \\
\hline prAtg39_Xbal & TCATCTAGACTGTAGTTTCAAAAACTGTGCTCC \\
\hline Atg39_nonstop_Smal & TAGCCCGGGGTGTTTGCACTTAAAATAATTTTTTTTCC \\
\hline prNvj1_BamHI_fw & TCAGGATCCCCACGCTGCTATGGAAG \\
\hline Nvj1_nonstop_EcoRI_rev & TAGGAATTCGTATCTAAATGGTTGAGAGTATGC \\
\hline \multirow[t]{2}{*}{ LEU2_nat_fw } & TTAAGCAAGGATTTTCTTAACTTCTTCGGCGACAGCAT \\
\hline & CACCGACTTCGGTGGCGTACGCTGCAGGTCGAC \\
\hline \multirow[t]{2}{*}{ LEU2_nat_fw } & ATGTCTGCCCCTAAGAAGATCGTCGTTTTGCCAGGTG \\
\hline & ACCACGTTGGTCAAGATCGATGAATTCGAGCTCG \\
\hline \multirow[t]{2}{*}{ Nvj1_KO_fw } & TGTGCATAATATCAAAAAAGCTACAAATATAATTGTAAA \\
\hline & ATATAATAAGCATGCGTACGCTGCAGGTCGAC \\
\hline \multirow[t]{2}{*}{ Nvj1_KO_rev } & CACCTCGTTGTAAGTGACGATGATAACCGAGATGACG \\
\hline & GAAATATAGTACATTAATCGATGAATTCGAGCTCG \\
\hline \multirow[t]{2}{*}{ Pep4_KO_fw } & GTGACCTAGTATTTAATCCAAATAAAATTCAAACAAAAA \\
\hline & CCAAAACTAACATGCGTACGCTGCAGGTCGAC \\
\hline \multirow[t]{2}{*}{ Pep4_KO_rev } & СTCTCTAGATGGCAGAAAAGGATAGGGCGGAGAAGT \\
\hline & AAGAAAAGTTTAGCTTAATCGATGAATTCGAGCTCG \\
\hline
\end{tabular}




\subsubsection{Antibodies}

Table 3.6: Antibodies applied in this study

Antibodies above the line were used for Western blotting those below the line were used for indirect immune fluorescence application.

\begin{tabular}{|c|c|c|}
\hline Antibody & Isotype/Host/Specificity & Reference \\
\hline \multirow[t]{2}{*}{ Anti-GFP } & $\operatorname{lgG} 1 \kappa$, mouse, anti-GFP & Roche Diagnostics GmbH, \\
\hline & & Mannheim, Germany \\
\hline \multirow[t]{2}{*}{ Anti-PGK1 } & IgG1k, mouse, anti-PGK1 & Thermo Fisher Scientific GmbH, \\
\hline & & Bremen, Germany \\
\hline \multirow[t]{2}{*}{ Anti-mouse } & IgG, goat, anti-mouse lgG & Dianova GmbH, Hamburg, \\
\hline & $(\mathrm{H}+\mathrm{L})-\mathrm{HRPO}$-conjugate & Germany \\
\hline FluoTag ${ }^{\circledR}-\mathrm{Q}$ anti- & sdAb, camelid, anti-GFP & NanoTag Biotechnologies \\
\hline GFP Atto647N & & GmbH, Göttingen, Germany \\
\hline
\end{tabular}

\subsubsection{Equipment}

Table 3.7: Equipment used in this study

\begin{tabular}{|c|c|}
\hline Equipment & Reference \\
\hline \multirow[t]{2}{*}{ Agarose gel chamber Mini-Sub ${ }^{\circledR}$ cell GT } & Bio-Rad Laboratories GmbH, \\
\hline & München, Germany \\
\hline \multirow[t]{2}{*}{ Agarose gel chamber wide Mini-Sub ${ }^{\circledR}$ cell GT } & Bio-Rad Laboratories GmbH, \\
\hline & München, Germany \\
\hline Amersham ${ }^{\mathrm{TM}}$ HybondTM PVDF blotting & GE Healthcare $\mathrm{GmbH}$, Solingen, \\
\hline membrane & Germany \\
\hline Autoclave Systec DX-200 & Systec GmbH, Linden, Germany \\
\hline BioPhotometer 6131 spectrophotometer & Eppendorf AG, Hamburg, Germany \\
\hline
\end{tabular}




\begin{tabular}{|c|c|}
\hline Equipment & Reference \\
\hline \multirow[t]{2}{*}{ Blot Shaker GFL ${ }^{\circledR} 3019$} & GFL Gesellschaft für Labortechnik \\
\hline & mbH, Burgwedel, Germany \\
\hline \multirow[t]{3}{*}{$\mathrm{CAMAG}^{\circledR}$ flat bottom chamber $20 \times 20 \mathrm{~cm}$} & Camag Chemie-Erzeugnisse und \\
\hline & Adsorptionstechnik AG \& Co. GmbH, \\
\hline & Berlin, Germany \\
\hline \multirow{2}{*}{ Camera CoolSNAP $\mathrm{HO}^{\mathrm{TM}}$} & Roper Scientific GmbH, Martinsried, \\
\hline & Germany \\
\hline Camera EMCCD & Andor, Belfast, United Kingdom \\
\hline Centrifuge 5415D & Eppendorf AG, Hamburg, Germany \\
\hline Centrifuge 5415R & Eppendorf AG, Hamburg, Germany \\
\hline Centrifuge $5417 \mathrm{C}$ & Eppendorf AG, Hamburg, Germany \\
\hline Centrifuge 5804 & Eppendorf AG, Hamburg, Germany \\
\hline Centrifuge $5804 \mathrm{R}$ & Eppendorf AG, Hamburg, Germany \\
\hline \multirow[t]{2}{*}{ Clean Bench } & BDK Luft- und Reinraumtechnik $\mathrm{GmbH}$, \\
\hline & Sonnenbühl-Genkingen, Germany \\
\hline Consort power supply E831 & Consort BVBA, Turnhout, Belgium \\
\hline Corning $^{\mathrm{TM}}$ Stripettor ${ }^{\mathrm{TM}}$ Ultra Pipet Controller & Corning Inc., Corning, New York, USA \\
\hline \multirow[t]{2}{*}{ Cuvettes no. $67.742(10 \times 4 \times 45 \mathrm{~mm})$} & Sarstedt AG \& Co. KG, Nümbrecht, \\
\hline & Germany \\
\hline DeltaVision ${ }^{\circledR}$ Core fluorescence microscope & Applied Precision, Issaquah, \\
\hline setup & Washington, USA \\
\hline Diagnostic microscope slides, 8 well $6 \mathrm{~mm}$ & Thermo Fisher Scientific GmbH, \\
\hline numbered & Bremen, Germany \\
\hline Diaphragm vacuum pump & Vacuubrand GmbH \& Co. KG \\
\hline
\end{tabular}




\begin{tabular}{|c|c|}
\hline Equipment & Reference \\
\hline Dispensette $^{\circledR} 25 \mathrm{~mL}$ & $\begin{array}{l}\text { BRAND GmbH \& Co. KG, Wertheim, } \\
\text { Germany }\end{array}$ \\
\hline Disruptor Genie ${ }^{\circledR}$ Vortex-Shaker & $\begin{array}{l}\text { Scientific Industries Inc., Bohemia, New } \\
\text { York, USA }\end{array}$ \\
\hline easy3D STED microscopy setup & $\begin{array}{l}\text { Abberior Instruments } \mathrm{GmbH} \text {, } \\
\text { Göttingen, Germany }\end{array}$ \\
\hline Eppendorf Safe-Lock tubes, $1.5 \mathrm{~mL}$ & Eppendorf AG, Hamburg, Germany \\
\hline Eppendorf Safe-Lock tubes, $2.0 \mathrm{~mL}$ & Eppendorf AG, Hamburg, Germany \\
\hline Freezer $\left(-20^{\circ} \mathrm{C}\right)$ & Liebherr, Bulle, Switzerland \\
\hline Freezer $\left(-80^{\circ} \mathrm{C}\right)$ & $\begin{array}{l}\text { Heraeus Holding GmbH, Hanau, } \\
\text { Germany }\end{array}$ \\
\hline Gasprofi 1 SCS micro & $\begin{array}{l}\text { WLD-TEC GmbH, Arenshausen, } \\
\text { Germany }\end{array}$ \\
\hline Glass beads, acid washed (425 - $600 \mu \mathrm{m})$ & Merck KGaA, Darmstadt, Germany \\
\hline $\begin{array}{l}\text { HPTLC silica gel } 60 F_{254} \text { glass plates } 20 \times \\
20 \mathrm{~cm}\end{array}$ & Merck KGaA, Darmstadt, Germany \\
\hline Innova 4200 Incubator-Shaker $\left(37^{\circ} \mathrm{C}\right)$ & $\begin{array}{l}\text { New Brunswick Scientific GmbH, } \\
\text { Nürtingen, Germany }\end{array}$ \\
\hline JULABO MA-4 heating circulator & Julabo GmbH, Seelbach, Germany \\
\hline Lab pH meter inoLab ${ }^{\circledR}$ pH 7110 & $\begin{array}{l}\text { Xylem Analytics Germany Sales GmbH } \\
\text { \& Co. KG, Weilheim, Germany }\end{array}$ \\
\hline Lab shaker LS-X & $\begin{array}{l}\text { Kühner Shaker GmbH, Herzogenrath, } \\
\text { Germany }\end{array}$ \\
\hline Lab shaker SBM/SS-X (Rack-Shaker) & $\begin{array}{l}\text { Kühner Shaker GmbH, Herzogenrath, } \\
\text { Germany }\end{array}$ \\
\hline
\end{tabular}




\begin{tabular}{|c|c|}
\hline Equipment & Reference \\
\hline \multirow[t]{2}{*}{ LAS-3000 Intelligent Dark Box } & FUJIFILM Europe GmbH, Düsseldo \\
\hline & Germany \\
\hline \multirow[t]{2}{*}{ Magnetic stirrer MR 2002} & Heidolph Elektro GmbH \& Co. KG, \\
\hline & Kelheim, Germany \\
\hline \multirow[t]{2}{*}{ Magnetic stirrer MR 3001} & Heidolph Elektro GmbH \& Co. KG, \\
\hline & Kelheim, Germany \\
\hline \multirow[t]{2}{*}{ Menzel $^{\mathrm{TM}}$ microscope coverslips $(24 \times 24 \mathrm{~mm})$} & Thermo Fisher Scientific GmbH, \\
\hline & Bremen, Germany \\
\hline Menzel $^{\mathrm{TM}}$ microscope slides $(76 \times 26 \mathrm{~mm})$, cut & Thermo Fisher Scientific GmbH, \\
\hline edges & Bremen, Germany \\
\hline \multirow[t]{2}{*}{ Microwave R-939 } & Sharp Electronics $\mathrm{GmbH}$, Hamburg \\
\hline & Germany \\
\hline \multirow[t]{2}{*}{ Mini Trans-Blot ${ }^{\circledR}$ cell } & Bio-Rad Laboratories GmbH, \\
\hline & München, Germany \\
\hline Mini-PROTEAN ${ }^{\circledR}$ Tetra Vertical & Bio-Rad Laboratories GmbH, \\
\hline electrophoresis cell & München, Germany \\
\hline Minisart ${ }^{\circledR}$ filters (pore size $0.2 \mu \mathrm{m}$ ) & Sartorius AG, Göttingen, Germany \\
\hline Nalgene ${ }^{\circledR}$ Rapid-Flow ${ }^{\mathrm{TM}} 75 \mathrm{~mm}$ bottle top & Thermo Fisher Scientific GmbH, \\
\hline filter, $500 \mathrm{~mL}$ & Bremen, Germany \\
\hline \multirow[t]{2}{*}{ NanoVue ${ }^{\mathrm{TM}}$ UV/Visible spectrophotometer } & GE Healthcare GmbH, Solingen, \\
\hline & Germany \\
\hline Olympus IX71 microscope & Olympus Corp., Shinjuku, Japan \\
\hline Olympus IX83 microscope & Olympus Corp., Shinjuku, Japan \\
\hline \multirow[t]{2}{*}{ Savant $^{\mathrm{TM}}$ SpeedVac ${ }^{\mathrm{TM}}$ Concentrator } & Thermo Fisher Scientific GmbH, \\
\hline & Bremen, Germany \\
\hline
\end{tabular}




\begin{tabular}{|c|c|}
\hline Equipment & Reference \\
\hline PCR Mastercycler ${ }^{\circledR}$ gradient & Eppendorf AG, Hamburg, Germany \\
\hline \multirow[t]{2}{*}{ Pipette controllers accu-jet ${ }^{\circledR}$ pro } & BRAND GmbH \& Co. KG, Wertheim, \\
\hline & Germany \\
\hline Pipettes Eppendorf Research ${ }^{\circledR}$ plus & Eppendorf AG, Hamburg, Germany \\
\hline \multirow[t]{2}{*}{ PowerPac ${ }^{\mathrm{TM}}$ Basic Power Supply } & Bio-Rad Laboratories GmbH, \\
\hline & München, Germany \\
\hline \multirow[t]{2}{*}{ PowerPac ${ }^{\mathrm{TM}}$ HC Power Supply } & Bio-Rad Laboratories GmbH, \\
\hline & München, Germany \\
\hline Refrigerator $\left(4^{\circ} \mathrm{C}\right)$ & Liebherr, Bulle, Switzerland \\
\hline \multirow[t]{2}{*}{ Roto Shake Genie ${ }^{\circledR}$} & Scientific Industries Inc., Bohemia, New \\
\hline & York, USA \\
\hline Sartorius Handy H51-D lab balance & Sartorius AG, Göttingen, Germany \\
\hline Sartorius Universal U4100 lab balance & Sartorius AG, Göttingen, Germany \\
\hline \multirow[t]{2}{*}{ SenTix ${ }^{\circledR} 41$ PLUS pH electrode } & Xylem Analytics Germany Sales GmbH \\
\hline & \& Co. KG, Weilheim, Germany \\
\hline \multirow[t]{2}{*}{ Shaker VXR basic Vibrax ${ }^{\circledR}$} & IKA ${ }^{\circledR}-$ Werke GmbH \& Co. KG, Staufen, \\
\hline & Germany \\
\hline \multirow[t]{2}{*}{ Shaking Water Bath SWB25 } & Thermo Haake GmbH, Karlsruhe, \\
\hline & Germany \\
\hline \multirow[t]{2}{*}{ Sprout ${ }^{\circledR}$ Mini Centrifuge } & Heathrow Scientific, Vernon Hills, \\
\hline & Illinois, USA \\
\hline ThermoMixer ${ }^{\circledR}$ comfort & Eppendorf AG, Hamburg, Germany \\
\hline Transilluminator TI1 & Biometra GmbH, Göttingen, Germany \\
\hline Transilluminator UVsolo & Biometra GmbH, Göttingen, Germany \\
\hline
\end{tabular}




\begin{tabular}{ll}
\hline Equipment & Reference \\
\hline $\begin{array}{l}\text { UPlanSApo } \times 100,1.4 \text { NA, oil immersion } \\
\text { objective }\end{array}$ & Olympus Corp., Shinjuku, Japan \\
Vortex Genie ${ }^{\circledR} 2$ & Scientific Industries Inc., Bohemia, New \\
& York, USA \\
Whatman $^{\mathrm{TM}}$ Gel Blot Paper & GE Healthcare GmbH, Solingen, \\
& Germany
\end{tabular}

\subsubsection{Commercially available kits}

Ready-to-use kits and commercial experiment solutions listed in Table 3.8, were used according to manufacturer's instructions unless specified otherwise.

Table 3.8: Commercial kit solutions used in this study

\begin{tabular}{|c|c|}
\hline Kit & Reference \\
\hline Amersham ${ }^{\mathrm{TM}} \mathrm{ECL} \mathrm{TM}^{\mathrm{TM}}$ Western-Blotting & GE Healthcare GmbH, Solingen, Germany \\
\hline \multicolumn{2}{|l|}{ Detection Reagents } \\
\hline \multirow[t]{2}{*}{ DreamTaq $^{\mathrm{TM}}$ Green PCR Master Mix (2x) } & Thermo Fisher Scientific GmbH, Bremen, \\
\hline & Germany \\
\hline \multirow[t]{2}{*}{ NucleoSpin ${ }^{\circledR}$ Microbial DNA } & Macherey-Nagel GmbH \& Co. KG, Düren, \\
\hline & Germany \\
\hline Pierce ${ }^{\mathrm{TM}} \mathrm{ECL}$ Plus Western-Blotting & Thermo Fisher Scientific GmbH, Bremen, \\
\hline Substrate & Germany \\
\hline QIAquick ${ }^{\circledR}$ Gel Extraction Kit & Qiagen, Hilden, Germany \\
\hline QIAquick ${ }^{\circledR}$ PCR Purification Kit & Qiagen, Hilden, Germany \\
\hline QuikChange ${ }^{\circledR}$ II Site-Directed & Agilent Technologies, Inc., Santa Clara, \\
\hline Mutagenesis Kit & California, USA \\
\hline
\end{tabular}




\begin{tabular}{ll}
\hline Kit & Reference \\
\hline QuikChange ${ }^{\circledR}$ Lightning Site-Directed & Agilent Technologies, Inc., Santa Clara, \\
Mutagenesis Kit & California, USA \\
Wizard ${ }^{\circledR}$ Plus SV Miniprep System & Promega GmbH, Mannheim, Germany
\end{tabular}

\subsubsection{Chemicals and consumables}

Chemicals were obtained from Carl Roth GmbH \& Co. KG (Karlsruhe, Germany) or Merck KGaA (Darmstadt, Germany) unless stated otherwise.

Table 3.9: Chemicals and consumables used in this study

\begin{tabular}{|c|c|}
\hline Chemical/consumable & Reference \\
\hline \multirow[t]{2}{*}{ Bacto $^{\mathrm{TM}}$ agar } & Becton Dickinson GmbH, Heidelberg, \\
\hline & Germany \\
\hline \multirow[t]{2}{*}{ Bacto $^{T M}$ pepton } & Becton Dickinson GmbH, Heidelberg, \\
\hline & Germany \\
\hline \multirow[t]{2}{*}{ Bacto $^{\mathrm{TM}}$ trypton } & Becton Dickinson $\mathrm{GmbH}$, Heidelberg, \\
\hline & Germany \\
\hline \multirow[t]{2}{*}{ Bacto $^{\mathrm{TM}}$ yeast extract } & Becton Dickinson GmbH, Heidelberg, \\
\hline & Germany \\
\hline \multirow[t]{2}{*}{ Coomassie Brilliant Blue G 250} & Serva Electrophoresis GmbH, Heidelberg, \\
\hline & Germany \\
\hline DeltaVision ${ }^{\circledR}$ immersion oil $(\mathrm{N}=1.520)$ & GE Healthcare $\mathrm{GmbH}$, Solingen, Germany \\
\hline Difco $^{\mathrm{TM}}$ yeast nitrogen base $\mathrm{w} / \mathrm{o}$ amino & Becton Dickinson GmbH, Heidelberg, \\
\hline acids & Germany \\
\hline Difco $^{\mathrm{TM}}$ yeast nitrogen base w/o amino & Becton Dickinson GmbH, Heidelberg, \\
\hline acids and ammonium sulphate & Germany \\
\hline
\end{tabular}




\begin{tabular}{|c|c|}
\hline Chemical/consumable & Reference \\
\hline Frema Reform instant skim milk powder & Granovita GmbH, Heimertingen, Germany \\
\hline \multirow[t]{2}{*}{ Gel loading dye, purple $(6 \times)$} & New England Biolabs GmbH, Frankfurt am \\
\hline & Main, Germany \\
\hline Herring sperm DNA & Promega GmbH, Mannheim, Germany \\
\hline Invitrogen ${ }^{\mathrm{TM}}$ Molecular Probes ${ }^{\mathrm{TM}}$ FM 4-64 & Thermo Fisher Scientific GmbH, Bremen, \\
\hline Dye & Germany \\
\hline Invitrogen ${ }^{\mathrm{TM}}$ Molecular Probes ${ }^{\mathrm{TM}}$ Hoechst & Thermo Fisher Scientific GmbH, Bremen, \\
\hline 33342 & Germany \\
\hline Normal Goat Serum & Abcam, Cambridge, United Kingdom \\
\hline Nourseothricin-dihydrogen sulphate/ & Werner BioAgents GmbH, Jena, Germany \\
\hline \multicolumn{2}{|l|}{ clonNAT powder } \\
\hline Phalloidin-iFluor ${ }^{\mathrm{TM}} 488$ reagent & Abcam, Cambridge, United Kingdom \\
\hline \multirow[t]{2}{*}{ Ponceau S } & Serva Electrophoresis GmbH, Heidelberg, \\
\hline & Germany \\
\hline Precision Plus Protein ${ }^{\mathrm{TM}}$ All Blue & Bio-Rad Laboratories GmbH, München, \\
\hline prestained protein standard & Germany \\
\hline \multirow[t]{2}{*}{ Rapamycin } & LC Laboratories, Woburn, Massachusetts, \\
\hline & USA \\
\hline \multirow[t]{2}{*}{ TriDye $^{\mathrm{TM}} 1 \mathrm{~kb}$ DNA ladder } & New England Biolabs GmbH, Frankfurt am \\
\hline & Main, Germany \\
\hline
\end{tabular}




\subsubsection{Enzymes}

Table 3.10: Enzymes used in this study

\begin{tabular}{ll}
\hline Enzyme & Reference \\
\hline Alkaline Phosphatase, Calf Intestinal & $\begin{array}{l}\text { New England Biolabs GmbH, Frankfurt am } \\
\text { Main, Germany }\end{array}$ \\
KOD Hot Start DNA polymerase & Merck KGaA, Darmstadt, Germany \\
Restriction enzymes & New England Biolabs GmbH, Frankfurt am \\
& Main, Germany \\
T4 DNA ligase & New England Biolabs GmbH, Frankfurt am \\
& Main, Germany
\end{tabular}

\subsubsection{Media}

Table 3.11: Media used in this study

Media listed above the line were used for cultivation of $S$. cerevisiae, media listed below the line were used for cultivation of $E$. coli.

\begin{tabular}{|c|c|c|c|}
\hline Medium & Composition & & Reference \\
\hline \multirow[t]{3}{*}{ YPD medium (pH 5.5) } & $1 \%(w / v)$ & Bacto $^{\mathrm{TM}}$ yeast extract & Sherman (2002) \\
\hline & $2 \%(w / v)$ & Bacto $^{\mathrm{TM}}$ peptone & \\
\hline & $2 \%(w / v)$ & D-glucose & \\
\hline \multirow[t]{3}{*}{$\mathrm{CM}$ medium (pH 5.6) } & $0.67 \%(w / v)$ & $\begin{array}{l}\text { Yeast nitrogen base w/o } \\
\text { amino acids }\end{array}$ & Kaiser et al. (1994) \\
\hline & $2 \%(w / v)$ & D-glucose & \\
\hline & $0.2 \%(w / v)$ & Dropout mix & \\
\hline \multirow[t]{4}{*}{$\begin{array}{l}\mathrm{CM} \text { medium w/o } \\
\text { methionine }(\mathrm{pH} 5.6)\end{array}$} & $0.67 \%(w / v)$ & $\begin{array}{l}\text { Yeast nitrogen base w/o } \\
\text { amino acids }\end{array}$ & Kaiser et al. (1994) \\
\hline & $2 \%(w / v)$ & D-glucose & \\
\hline & $0.2 \%(w / v)$ & Dropout mix w/o & \\
\hline & & methionine & \\
\hline
\end{tabular}




\begin{tabular}{|c|c|c|c|}
\hline Medium & Composition & & Reference \\
\hline \multirow[t]{2}{*}{ SD-N medium } & $0.67 \%(w / v)$ & $\begin{array}{l}\text { Yeast nitrogen base w/o } \\
\text { amino acids and w/o } \\
\text { ammonium sulphate }\end{array}$ & $\begin{array}{l}\text { Takeshige et al. } \\
\text { (1992) }\end{array}$ \\
\hline & $2 \%(w / v)$ & D-glucose & \\
\hline \multirow[t]{3}{*}{ LB medium (pH 7.5) } & $1 \%(w / v)$ & Bacto $^{\mathrm{TM}}$ trypton & Bertani (1951) \\
\hline & $0.5 \%(w / v)$ & Bacto $^{\mathrm{TM}}$ yeast extract & \\
\hline & $0.5 \%(w / v)$ & Sodium chloride & \\
\hline \multirow[t]{7}{*}{ SOC medium ( $\mathrm{pH} 7.5)$} & $2 \%(w / v)$ & Bacto $^{\mathrm{TM}}$ trypton & Hanahan (1983) \\
\hline & $0.5 \%(w / v)$ & Bacto $^{\mathrm{TM}}$ yeast extract & \\
\hline & $0.4 \%(w / v)$ & D-glucose & \\
\hline & $10 \mathrm{mM}$ & Sodium chloride & \\
\hline & $10 \mathrm{mM}$ & Magnesium sulphate & \\
\hline & $10 \mathrm{mM}$ & Magnesium chloride & \\
\hline & $2.5 \mathrm{mM}$ & Potassium chloride & \\
\hline
\end{tabular}

\subsubsection{Buffers and solutions}

Table 3.12: Buffers and solutions used in this study

\begin{tabular}{lll}
\hline Buffer/solution & \multicolumn{2}{l}{ Composition } \\
\hline TAE buffer & $40 \mathrm{mM}$ & Tris \\
$20 \mathrm{mM}$ & Acetic acid \\
$2 \mathrm{mM}$ & EDTA \\
\hline PEG in LiTE buffer $(\mathrm{pH} \mathrm{8.0)}$ & $100 \mathrm{mM}$ & Lithium acetate \\
& $10 \mathrm{mM}$ & Tris/acetic acid \\
$1 \mathrm{mM}$ & EDTA \\
$40 \%(\mathrm{w} / \mathrm{v})$ & PEG 3350
\end{tabular}




\begin{tabular}{|c|c|c|}
\hline Buffer/solution & Composition & \\
\hline \multirow[t]{4}{*}{ LiOAc-Sorb buffer (pH 8.0) } & $100 \mathrm{mM}$ & Lithium acetate \\
\hline & $10 \mathrm{mM}$ & Tris/acetic acid \\
\hline & $1 \mathrm{mM}$ & EDTA \\
\hline & $1 \mathrm{M}$ & D-sorbitol \\
\hline \multirow[t]{2}{*}{ Lysis buffer } & $1.85 \mathrm{M}$ & Sodium hydroxyde \\
\hline & $20 \mathrm{mM}$ & DTT \\
\hline \multirow[t]{4}{*}{ SDS sample buffer ( $\mathrm{pH} 7.5)$} & $100 \mathrm{mM}$ & Tris/hydrochloric acid \\
\hline & $2 \%(w / v)$ & SDS \\
\hline & $20 \mathrm{mM}$ & DTT \\
\hline & $10 \%(v / v)$ & Glycerol \\
\hline \multirow[t]{3}{*}{ SDS running buffer } & $25 \mathrm{mM}$ & Tris \\
\hline & $200 \mathrm{mM}$ & Glycine \\
\hline & $0.1 \%(w / v)$ & SDS \\
\hline \multirow[t]{3}{*}{ Western blotting buffer } & $192 \mathrm{mM}$ & Glycine \\
\hline & $25 \mathrm{mM}$ & Tris \\
\hline & $20 \%(v / v)$ & Ethanol \\
\hline \multirow[t]{3}{*}{ TBST buffer (pH 7.6) } & $20 \mathrm{mM}$ & Tris/hydrochloric acid \\
\hline & $137 \mathrm{mM}$ & Sodium chloride \\
\hline & $0.1 \%(w / v)$ & Tween ${ }^{\circledR} 20$ \\
\hline \multirow[t]{4}{*}{$1 \times P B S(p H 7.4)$} & $140 \mathrm{mM}$ & Sodium chloride \\
\hline & $2.7 \mathrm{mM}$ & Potassium chloride \\
\hline & $10 \mathrm{mM}$ & Disodium phosphate \\
\hline & $1.8 \mathrm{mM}$ & Monopotassium phosphate \\
\hline
\end{tabular}




\subsection{Methods}

All methods performed in this section are based on methods described by Treco \& Winston (2008) and Sambrook et al. (1989), unless declared otherwise.

\subsubsection{Cultivation of E. coli}

E. coli strains $\mathrm{XL1}$-Blue and $\mathrm{DH} 5 \alpha\left(\mathrm{NEB}^{\circledR}\right.$ 5-alpha) were used for molecular biological methods. E. coli cultures were grown in liquid lysogeny broth (LB) medium or on LB agar plates, both supplemented for plasmid selection with ampicillin (Amp) as the designated antibiotic for all plasmid/E. coli strain combinations used in this work. Liquid cultures were incubated at $37^{\circ} \mathrm{C}$ on an Innova 4200 shaker, set to $220 \mathrm{rpm}$. Selection for plasmid positive clones was performed by spreading of transformed E. coli cells on LB agar supplemented with the appropriate antibiotic and subsequent incubation $\mathrm{O} / \mathrm{N}$ at $37^{\circ} \mathrm{C}$.

Cryo-stocks were prepared using appropriate growth medium containing 30\% (v/ v) glycerol and stored at $-80^{\circ} \mathrm{C}$.

\subsubsection{Cultivation of S. cerevisiae}

Yeast strains were cultivated under nutrient-rich conditions in yeast peptone dextrose (YPD) medium. Selection for auxotrophy markers was performed in complete minimal (CM) medium, supplemented with the appropriate amino acids. Yeast cultures were cultivated at $30^{\circ} \mathrm{C}$ shaking. Cell density was determined photometrically measuring the $\mathrm{OD}_{600}$. Pre-cultures were grown $\mathrm{O} / \mathrm{N}$ until stationary phase and then inoculated for growth of main-cultures. Dilutions were inoculated in accordance to the targeted growth phase. 
Storage of yeast cells was realised using cryo-stocks, by freezing stationary cells to $-80^{\circ} \mathrm{C}$ in appropriate medium, containing $15 \%(\mathrm{v} / \mathrm{v})$ glycerol.

\subsubsection{Molecular biological methods}

\subsubsection{Isolation of chromosomal DNA from yeast cells}

Chromosomal DNA was isolated from yeast cells using the NucleoSpin ${ }^{\circledR}$ Microbial DNA kit. A volume of $2 \mathrm{~mL}$ of a yeast $\mathrm{O} / \mathrm{N}$ culture was harvested and processed according to manufacturer's instructions.

\subsubsection{Polymerase chain reaction}

For amplification of DNA fragments polymerase chain reaction (PCR) was performed using appropriate oligonucleotide primers flanking the target sequence. PCR was performed on a Mastercycler ${ }^{\circledR}$ Gradient using DreamTaq ${ }^{\mathrm{TM}}$ polymerase for control PCRs to verify genomic modifications, and KOD Hot Start DNA polymerase for generation of target DNA fragments used for transformation or genomic integration. PCRs were performed according to the polymerases respective manufacturer's instructions, amplicon length and oligonucleotide primer specifications.

\subsubsection{DNA agarose gel electrophoresis}

Agarose gel electrophoresis was performed according to Aaij \& Borst (1972). DNA samples were prepared by addition of $6 \times$ gel loading dye to the DNA sample. Agarose gels were produced by boiling $0.8 \%(\mathrm{w} / \mathrm{v})$ agarose in an appropriate volume of TAE-buffer with the addition of $2 \mu \mathrm{g} / \mathrm{mL}$ ethidium bromide. Samples were then applied on the agarose gel alongside $5-10 \mu \mathrm{L}$ TriDye $^{\mathrm{TM}} 1 \mathrm{~kb}$ DNA ladder for size determination. Electrophoresis of the gel was 
subsequently performed until the bromophenol-blue band exited the agarose gel.

\subsubsection{Agarose gel extraction}

Gel extraction of fragments of interest was performed using the QlAquick ${ }^{\circledR}$ Gel Extraction Kit for agarose gels. Appropriate DNA fragments were cut from ethidium bromide stained agarose gels and processed in accordance with the manufacturer's protocol.

\subsubsection{Determination and adjustment of DNA concentration}

Concentrations of DNA were determined, prior to experimental application, using the NanoVue TM UV/Visible spectrophotometer. In case sample DNA concentrations did not meet experimental requirements, DNA was precipitated and resolved in an appropriately smaller volume. For precipitation 10\% (v/v) of $3 \mathrm{M}$ sodium acetate $(\mathrm{pH}$ 5.2) were added to the DNA sample. Afterwards 2.5 sample volumes of $98 \%$ ethanol were added and vortexed. DNA was then precipitated at $-80^{\circ} \mathrm{C}$ for $1 \mathrm{~h}$ and afterwards centrifuged at $20,000 \times \mathrm{g}$ and $4^{\circ} \mathrm{C}$ for 20 min. Pelleted DNA was washed twice with $70 \%$ ethanol and subsequently dried at $65^{\circ} \mathrm{C}$ until all residual ethanol was removed, and then resuspended in the suitable volume of $d d \mathrm{H}_{2} \mathrm{O}$.

\subsubsection{Restriction digestion of DNA}

Restriction of DNA for cloning was performed using restriction endonucleases and buffer systems available from New England Biolabs GmbH. High-fidelity $\left(\mathrm{HF}^{\circledR}\right)$ versions of the restriction endonucleases were used, if available. All restriction digestions were performed according to manufacturer's instructions. 


\subsubsection{Molecular cloning}

Plasmid constructs generated for this work are listed in Table 3.1.2. Cloning was performed, generating sequences of target ORF with and without promoter or terminator sequences up- and downstream of the ORF using oligonucleotides in combination with DNA from the WCG4a wildtype (wt) strain. Oligonucleotides were designed in a way, that target insert DNA was bracketed by sequences appropriate for cutting with select restriction enzymes according to Boyer (1971). PCR products were verified by DNA electrophoresis and purified using the QlAquick ${ }^{\circledR}$ Gel Extraction Kit. Approx. 5 - $10 \mu \mathrm{g}$ of PCR products and target plasmids were digested with the appropriate restriction enzymes according to manufacturer's instructions. To counteract religation, $1 \mu$ of calf intestinal phosphatase was added to the reaction of the vector. Concentrations of purified products were determined and used for a ligation reaction in an insert to vector molar ratio of 5:1. Ligation was performed using the T4 DNA ligase (Weiss \& Richardson, 1967) according to manufacturer's instructions. Chemically competent XL1-Blue cells were subsequently transformed with the entire volume of the ligation reaction. All products of molecular cloning were verified by sequencing.

\subsubsection{Transformation of plasmid DNA in chemically competent E. coli}

Competent E. coli cells were transformed by heat-shock according to the method described by Mandel \& Higa (1970). Approx. $0.1-1 \mu \mathrm{g}$ of DNA in a max. volume of $10 \mu \mathrm{L}$, or $10 \mu \mathrm{L}$ of ligation setup were applied to $50 \mu \mathrm{L}$ of competent $E$. coli cells and incubated on ice for $20 \mathrm{~min}$. Heat shock was achieved by exposure of competent cells to $42^{\circ} \mathrm{C}$ for $45 \mathrm{~s}$. Heat shock was followed by immediate incubation on ice for $5 \mathrm{~min}$ before addition of $900 \mu \mathrm{L}$ super optimal broth with catabolite repression (SOC) medium and successive incubation at $37^{\circ} \mathrm{C}$ for $1 \mathrm{~h}$ while shaking. The samples were spread out on LB-agarose plates containing the 
appropriate antibiotic. And plates were ultimately incubated $\mathrm{O} / \mathrm{N}$ at $37^{\circ} \mathrm{C}$.

\subsubsection{Purification of plasmids from E. coli}

Plasmid DNA preparation was performed as described by Birnboim \& Doly (1979) using the Wizard ${ }^{\circledR}$ Plus SV Miniprep System, according to manufacturer's instructions. Plasmids were isolated from $5 \mathrm{~mL} O / \mathrm{N}$ cultures grown in LB-medium containing the appropriate antibiotic, inoculated with a single E. coli colony.

\subsubsection{Sequencing of DNA}

All generated constructs were verified via sequencing. Sequencing was performed by Microsynth AG (Balgach, Switzerland). Samples of $12 \mu \mathrm{L}$ volume, containing 480 - $1200 \mathrm{ng}$ of purified plasmid DNA, were included alongside $30 \mathrm{pmol}$ of a suitable oligonucleotide primer. Evaluation of sequencing data was performed using SnapGene ${ }^{\circledR}$ 4.0.8.

\subsubsection{Yeast cell transformation}

High-efficiency transformation of yeast cells was performed applying the lithium acetate/single stranded carrier DNA/polyethylene glycol (PEG) method described by Gietz \& Schiestl (2007). A liquid culture of $50 \mathrm{~mL}$ was grown to an $\mathrm{OD}_{600}=0.5$ and subsequently harvested by centrifugation for $5 \mathrm{~min}$ at $500 \times \mathrm{g}$. Cells were washed twice with $d d_{2} \mathrm{H}_{2} \mathrm{O}$, and once with LiOAc-Sorb. Cells were then resuspended in $400 \mu \mathrm{L} \mathrm{LiOAc-Sorb}$ and incubated for $15 \mathrm{~min}$ at $30^{\circ} \mathrm{C}$. Aliquots of $100 \mu \mathrm{L}$ sample were added to $5 \mu \mathrm{L}$ single-stranded DNA (10 mg/mL), $10 \mu \mathrm{L}$ of target DNA with $300 \mu \mathrm{L}$ PEG in LiTE. The samples were subsequently incubated for $30 \mathrm{~min}$ at $30^{\circ} \mathrm{C}$ and heat-shocked for $15 \mathrm{~min}$ at $42^{\circ} \mathrm{C}$. Cells were sedimented and plated on the appropriate medium. 
Colonies were used for further experiments after incubation for 2 - 3 days. Genomic modifications were verified by extraction of the target DNA and subsequent PCR for detection of the respective inserted cassettes.

\subsubsection{Genome engineering based on homologous recombination}

Genomic insertions and gene deletions were performed according to Longtine et al. (1998) and Janke et al. (2004). Amplified PCR cassettes or digested integrative plasmid fragments were transformed in yeast and selected for strains that integrated the target DNA by homologous recombination at the chromosomal gene locus of interest, facilitating deletion or integration of DNA sequences.

\subsubsection{Biochemical methods}

\subsubsection{Alkaline lysis of yeast cells}

A quantity equal to an $\mathrm{OD}_{600}=5$ of cells was harvested by centrifugation for $5 \mathrm{~min}$ at $4^{\circ} \mathrm{C}$ and $500 \times \mathrm{g}$. The supernatant was removed and the cell pellet resuspended in $1 \mathrm{~mL}$ ice cold $\mathrm{dd}_{2} \mathrm{O}$. Subsequently, $150 \mu \mathrm{L}$ Lysis buffer was added and mixed. After sample incubation on ice for $10 \mathrm{~min}, 150 \mu \mathrm{L}$ of $50 \%(\mathrm{v} / \mathrm{v})$ trichloroacetic acid was added, vortexed and again incubated for 10 min on ice. After centrifugation at $4^{\circ} \mathrm{C}$ for $10 \mathrm{~min}$ at $18,000 \times \mathrm{g}$ the supernatant was removed and washed three times with $-20^{\circ} \mathrm{C}$ acetone. Removal of acetone was then followed by drying of the sample pellet at $37^{\circ} \mathrm{C}$. The dried pellet was ultimately resuspended in freshly prepared SDS sample buffer.

\subsubsection{Discontinuous SDS-polyacrylamid gel electrophoresis}

Sodium dodecyl sulfate-polyacrylamid gel electrophoresis (SDS-PAGE) was performed according to Laemmli (1970). Protein samples were prepared by addition of SDS sample buffer and denaturation at $95^{\circ} \mathrm{C}$ for $5 \mathrm{~min}$. Sample 
volumes of $20 \mu \mathrm{L}$ were applied to a $12 \%$ SDS-gel alongside $5 \mu \mathrm{L}$ of Precision Plus Protein $^{\mathrm{TM}}$ All Blue prestained protein standard, as the molecular weight marker. The SDS-gel was then run on a Mini-PROTEAN ${ }^{\circledR}$ Tetra Vertical electrophoresis cell at $30 \mathrm{~mA}$ per gel and $300 \mathrm{~V}$ for approx. $45 \mathrm{~min}$.

\subsubsection{Immunoblotting}

Immunoblotting for quantification of protein levels was performed according to Towbin et al. (1979). Prior to assembly, a PVDF blotting membrane was activated in $98 \%(\mathrm{v} / \mathrm{v})$ ethanol. Blotting was performed in a Mini Trans-Blot ${ }^{\circledR}$ Cell. The blotting setup was supplied with a cooling element and a stirring bar and ultimately run for $10 \mathrm{~h}$ at $80 \mathrm{~V}$ and $75 \mathrm{~mA}$.

After blotting, the membrane was treated for 5 min with Ponceau $\mathrm{S}$ staining solution for blotting verification, destained with $\mathrm{dd}_{2} \mathrm{O}$ and subsequently blocked in $1 \times$ TBST $+10 \%(\mathrm{w} / \mathrm{v})$ semi-skimmed milk powder for $1 \mathrm{~h}$ at RT. The membrane was then incubated with a primary antibody for $3 \mathrm{~h}$ at RT and afterwards washed in $10 \mathrm{~mL} 1 \times$ TBST, three times for $10 \mathrm{~min}$, before incubation with the secondary antibody at RT for $1 \mathrm{~h}$. The membrane was then washed three times with $1 \times$ TBST for $10 \mathrm{~min}$ and subsequently treated with the Amersham ${ }^{\mathrm{TM}}$ ECL $^{\mathrm{TM}}$ Westernblotting detection reagents. Incubation was performed in the dark for approx. 10 min and subsequent development was performed on an LAS-3000 system. Detected signals were recorded at multiple timepoints for aquisition of an optimal signal-to-noise ratio and ultimately quantified using Fiji (Schindelin et al., 2012).

\subsubsection{Neutral lipid extraction from S. cerevisiae}

Lipid extraction was performed in a modified version of the method described by Heilmann et al. (2012). Yeast cell cultures of an $\mathrm{OD}_{600}=50$ were harvested at 
diauxic phase in $50 \mathrm{~mL}$ Falcon $^{\mathrm{TM}}$ tubes by centrifugation for $10 \mathrm{~min}$ at $300 \times \mathrm{g}$ and then resuspended in $1 \mathrm{~mL}$ of methanol. The samples were vortexed with glass beads (acid washed, 425 - $600 \mu \mathrm{m}$ ) for $15 \mathrm{~min}$. Afterwards $2 \mathrm{~mL}$ of hexane were added and the samples were vortexed for another $15 \mathrm{~min}$ before centrifugation for $10 \mathrm{~min}$ at $500 \times \mathrm{g}$. The upper organic phase was then transferred to new $2 \mathrm{~mL}$ reaction tubes and evaporated using the SpeedVac ${ }^{\mathrm{TM}}$ Concentrator. The samples were finally resolved in $200 \mu \mathrm{L}$ hexane and stored in glass-vials at $-20^{\circ} \mathrm{C}$.

\subsubsection{Thin layer chromatography}

Thin layer chromatography (TLC) was performed using a modified version of the method described by Heilmann et al. (2012). Neutral lipid extracts were applied to TLC plates in volumes of $50 \mu \mathrm{L}$. Dried plates were developed in an incubation chamber containing $100 \mathrm{~mL}$ of hexane:diethyl ether:acetic acid (80:20:1, v/v/v) as the running solvent. The chamber was saturated prior to running the TLC, by addition of the solvent approx. $3 \mathrm{~h}$ before adding the silica plate. TLC was stopped shortly before the solvent front reached the upper edge of the plate and was subsequently dried. Dried plates were then briefly soaked in $\mathrm{CuSO}_{4}$ and after again drying, heated to $180^{\circ} \mathrm{C}$.

\subsubsection{Fixation of $S$. cerevisiae cells}

Fixation of yeast cells was performed applying a modified protocol of the method published by Ries et al. (2012). For fixation, $50 \mu \mathrm{L}$ of $0.2 \%(\mathrm{v} / \mathrm{v})$ glutaraldehyde (GA) in $1 \times$ PBS were added to $\mathrm{OD}_{600}=1$ of yeast cells on an 8 well microscopy slide with each well having a diameter of $6 \mathrm{~mm}$. Fixation was performed for 15 min while gently shaking the samples and stopped by removal of the GA solution and subsequently quenched by applying $50 \mu \mathrm{L}$ of a $10 \mathrm{mg} / \mathrm{mL}$ solution of sodium borohydride in $1 \times$ PBS. Quenching was performed for another $15 \mathrm{~min}$ of 
shaking, before the solution was removed and replaced with $1 \times$ PBS. The cells were washed three times with $1 \times$ PBS for $15 \mathrm{~min}$ and afterwards stained for subsequent microscopy.

\subsubsection{Labelling of target proteins with nanobodies in S. cerevisiae}

Labelling of fluorescent proteins tags was performed using FluoTag ${ }^{\circledR}-\mathrm{Q}$ single domain Antibodies (sdAb), kindly provided by Dr. Felipe Opazo (University Medical Center, Göttingen) and Dr. Steffen Frey (NanoTag Biotechnologies $\mathrm{GmbH}$, Göttingen). Permeabilisation was performed applying a modified version of the protocol described by Ries et al. (2012) and Kaplan \& Ewers (2015).

Cells previously fixed according to the protocol established in this work were blocked and permeabilised by gentle shaking at RT for 60 min in $50 \mu \mathrm{L}$ of $1 \times \mathrm{PBS}$ containing $0.25 \%(\mathrm{v} / \mathrm{v})$ Triton $\mathrm{X}-100$ and $5 \%(\mathrm{v} / \mathrm{v})$ normal goat serum (NGS). Permeabilised cells were labelled after three subsequent washes with $1 \times$ PBS for 15 min. Labelling buffer containing 0.5\% (v/v) FluoTag ${ }^{{ }^{-}}-\mathrm{O}$ sdAb, $0.25 \%(\mathrm{v} / \mathrm{v})$ Triton X-100, 1\% (v/v) NGS in $1 \times$ PBS was subsequently applied for 90 min while shaking. Cells were ultimately washed another three times with $1 \times$ PBS and finally embedded in Mowiol ${ }^{\circledR}$ mounting medium for microscopy.

\subsubsection{Mowiol $^{\circledR}$ mounting medium preparation}

The Mowiol ${ }^{\circledR}$ mounting medium, used for embedding of fixed cells, was prepared

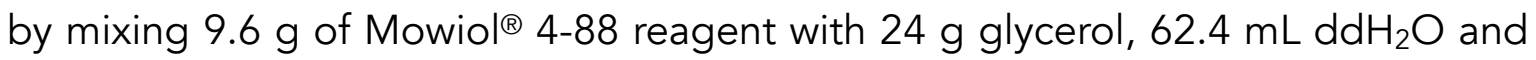
$9.6 \mathrm{~mL} 1 \mathrm{M}$ Tris buffer with a magnetic stirrer for 5 days. The dissolved mountant was subsequently used for cellular embedding, or stored at $4^{\circ} \mathrm{C}$. 


\subsubsection{Phalloidin staining}

F-actin staining with phalloidin was realised, using the Phalloidin-iFluor TM 488 conjugate. Staining with phalloidin was performed subsequent to fixation and permeabilisation procedures, analogous to the protocol, designed for protein decoration with sdAb. Phalloidin-iFluor ${ }^{\mathrm{TM}} 488$ was applied according to manufacturer's instructions, but adjusted to 30 min of incubation time at RT. With excitation and emission peaks at $495 \mathrm{~nm}$ and $518 \mathrm{~nm}$, Phalloidin-iFluor ${ }^{\mathrm{TM}} 488$ was recorded using the filter set, applied for detection of GFP.

\subsubsection{Widefield microscopy}

Live cell microscopy was performed using a DeltaVision ${ }^{\circledR}$ Core setup, based on the inverted IX71 microscope, equipped with the UPlanSApo $\times 100,1.4$ numerical aperture (NA), oil immersion objective and a CoolSNAP $\mathrm{HO}^{2 \mathrm{MM}}$ couple-charged device (CCD) camera.

Table 3.13: DeltaVision ${ }^{\circledR}$ filtersets applied for widefield microscopy

\begin{tabular}{l|ccc}
\hline \multirow{2}{*}{ Filter set } & \multicolumn{2}{c}{ Central wavelength/bandpass } & \\
\cline { 2 - 3 } & Excitation & Emission & Applied fluorophores \\
\hline Blue & $390 / 18 \mathrm{~nm}$ & $435 / 48 \mathrm{~nm}$ & mTagBFP \\
Green & $475 / 28 \mathrm{~nm}$ & $525 / 50 \mathrm{~nm}$ & GFP, yEGFP, Phalloidin- \\
Red & $575 / 25 \mathrm{~nm}$ & $632 / 69 \mathrm{~nm}$ & iFluor ${ }^{\text {TM }} 488$ \\
Far-red & $632 / 22 \mathrm{~nm}$ & $676 / 34 \mathrm{~nm}$ & Atto647N, LipidSpot
\end{tabular}

Stacks along the z-axis of 24 images, separated by $0.2 \mu \mathrm{m}$ distance, were obtained by sample excitation with an InsightSSI ${ }^{\mathrm{TM}}$ solid state illumination system and recording of emissions was realised by application of the appropriate filter 
set (Table 3.10). Differential interference contrast (DIC) images were recorded at the central stack of the cell, applying the DeltaVision ${ }^{\circledR}$ LED transmitted light source. Subsequent two dimensional (2D) and three dimensional (3D) evaluation was performed using softWoRx ${ }^{\circledR}$, Fiji and Huygens Professional.

\subsubsection{Confocal and stimulated emission depletion microscopy}

Confocal and stimulated emission depletion (STED) microscopy of fixed cells was realised on an inverse 4-channel easy3D STED setup, based on the IX83 microscope equipped with a Plan Apochromat $\times 100,1.4$ NA oil immersion objective and a EMCCD camera.

Confocal images were obtained applying the appropriate excitation lasers, pulsed at $40 \mathrm{MHz}$ and attenuated for imaging, applying an acousto optic tunable filter (AOTF). For GFP, a $488 \mathrm{~nm}$ excitation laser (AOTF set to $10 \%$ of $200 \mu \mathrm{W}$ max. power), for mCherry a $561 \mathrm{~nm}$ excitation laser (AOTF set to $20 \%$ of $440 \mu \mathrm{W}$ max. power) and Atto647N a $640 \mathrm{~nm}$ excitation laser (AOTF set to $20 \%$ of $1.77 \mathrm{~mW}$ max. power).

For STED imaging of Atto647N, the $640 \mathrm{~nm}$ excitation laser (set to $20 \%$ of $1.77 \mathrm{~mW}$ max. power) was applied and depletion was effected using a $775 \mathrm{~nm}$ STED laser (set to $10 \%$ of $1.25 \mathrm{~W}$ max. power) pulsed at $40 \mathrm{MHz}$. Scanning was performed with a dwell time of $15 \mu$ s per pixel at $20 \mathrm{~nm}$ pixel size. Emission was detected with two avalanche photodiode (APD) units at ranges $580-625 \mathrm{~nm}$ and $655-720 \mathrm{~nm}$. Operation of the setup and recording of images was performed using the Imspector 0.14 software. Evaluation and processing of the results was performed using Fiji and Huygens Professional. 


\section{Results}

In order to distinguish the different modes of nucleophagy with a high confidence, microscopic phenotype and cargo specificity were targeted as a potential means for gaining new insights into the different mechanisms for degradation of nuclear and nucleus associated cargo.

For microscopy, a system was required that allows for unambiguous identification of macro- versus micronucleophagy. By definition, microautophagy involves invagination of cargo by the vacuolar membrane. Pho8, a highly abundant vacuolar membrane resident protein, was used. An N-terminally tagged version, fused with the blue fluorescent protein (BFP), monomeric TagBFP (mTagBFP) was applied. The construct allows detection of the membrane, while preventing subjection of the tag to vacuolar proteases and thereby cleavage, an effect observed for its C-terminus, located in the lumen of the vacuole (Yoshinobu et al., 1987; Klionsky \& Emr, 1989). For visualisation of the nucleus and to ensure exclusive detection of nucleophagic processes, the NLS of Nab2 (NLS $\mathrm{Nab}_{2}$ ) was used in a monomeric Cherry (mCherry) fused construct.

Due to their comparable brightness, bleaching resistance and maturation time, relative to green fluorescent protein (GFP) and yeast optimised, enhanced GFP (yEGFP), a combination of GFP or yEGFP, with mTagBFP and mCherry was selected. Their distinct excitation and emission spectra allow for separate detection of all three fluorescent proteins on the employed microscopy setups (Subach et al., 2008; Shaner et al., 2004; Merzlyak et al., 2007; Yang et al., 1996; Sarkisyan et al., 2015). High detectability of GFP emission in both microscopy setups, favoured a setup of target proteins fused to GFP or yEGFP, for investigation of components of the autophagic machinery. Multiple tagging with $3 \times m$ TagBFP was used to compensate for weak signal intensity, yet high 
abundance of $\mathrm{NLS}_{\mathrm{Nab2}}-\mathrm{mCherry}$ in the nucleoplasm, did not require application of a similar strategy. Both $3 \times$ mTagBFP-Pho8 and $\mathrm{NLS}_{\mathrm{Nab}_{2}-\mathrm{mCherry}}$ were expressed under the constitutive PGK1 and TEF1 promoters, respectively, that share a constant activity pattern, ensuring consistent gene expression (Partow et al., 2010). Target members of the autophagic machinery were instead, if possible, expressed under their native promoters, ensuring appropriate expression levels, while avoiding potential mislocalisation as a result from overabundance.
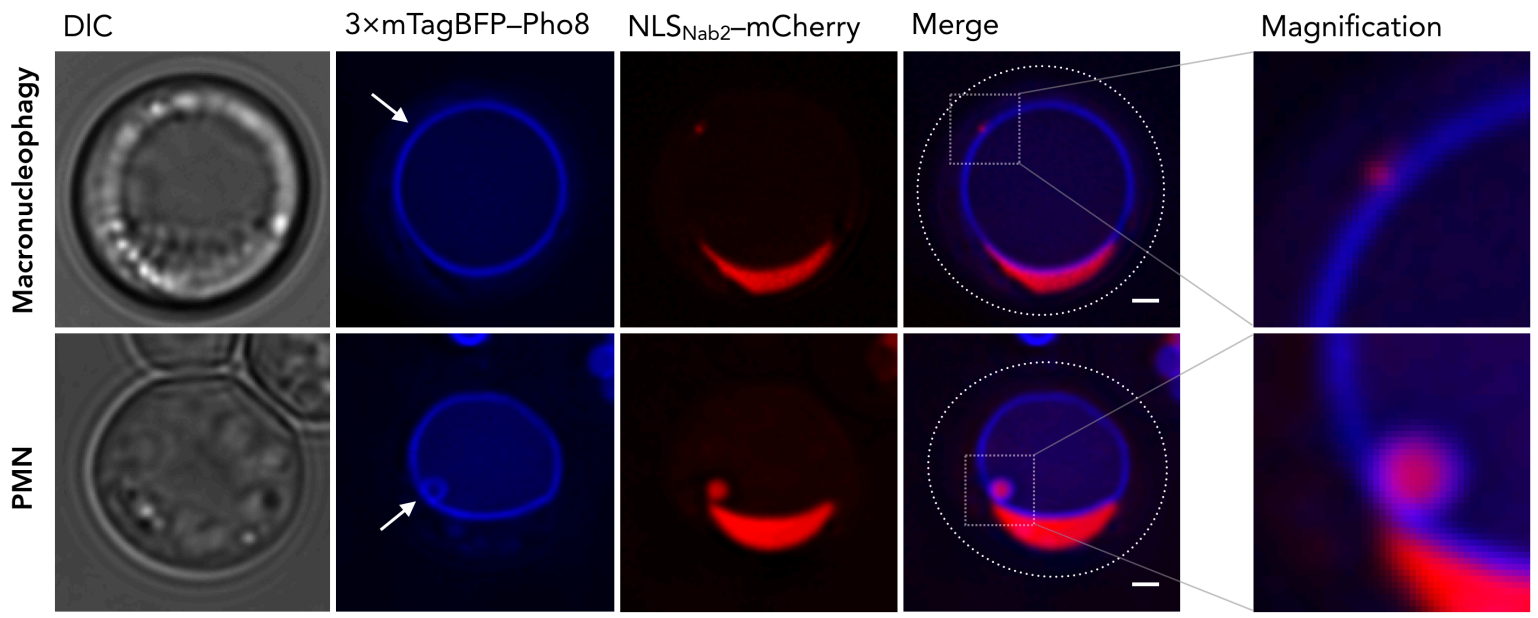

Figure 4.1: Distinction of the nucleophagic modes by fluorescence microscopy

Macronucleophagic cargo and PMN vesicles are depicted in fluorescence microscopy, by labelling the vacuolar membrane with $3 \times$ mTagBFP-Pho8 and the nucleoplasm with $\mathrm{NLS}_{\mathrm{Nab2}}$-mCherry. A differential interference contrast (DIC) image is shown and merged versions of the channels are additionally depicted. Merged images of nucleophagic cargo (micronuclei) are detailed as magnified $(\times 4)$ images. Arrows highlight the characteristic differences in appearance of the vacuolar membrane at sites of macro(upper panel) and micronucleophagic (lower panel) occurrence. Scale bars represent $1 \mu \mathrm{m}$.

Live cell microscopy was performed under starvation conditions. Yeast cells grown to early stationary phase were switched to synthetic defined medium, lacking nitrogen (SD-N), 2 - $4 \mathrm{~h}$ prior to microscopy. While various conditions were previously reported to result in an increased rate of nucleophagic structures, multi-channel microscopy of three different fluorescent proteins presented some restrictions. Prolonged periods of starvation coincided with an accumulation of vacuolar signal emission when using the DeltaVision ${ }^{\circledR}$ setup, effectively disturbing 
differentiation of vacuolar membrane from the lumen. This effect could be a result of progressive degradation of vacuolar membrane by microautophagy, presumably leading to a build-up of mTagBFP in the vacuolar lumen. Additionally, emission of light in the red channel was detected in the cytosol, when late stationary cells were used, a result possibly caused by a lack of nuclear retraction of the NLS containing protein, in senescent cells.

Detection of $3 \times$ mTagBFP-Pho8 and NLS $\mathrm{Nab2}_{2}-\mathrm{mCherry}$ allowed for morphological distinction of macro- and micronucleophagy by detection of absence or presence of $3 \times$ mTagBFP-Pho8 surrounding the cargo, respectively (Figure 4.1 ). Whereas (non-lumenar) PMN vesicles were consistently detected in close proximity to the main nucleus, macronucleophagic cargo was not restricted to the nuclear vacuolar contact site. Upon collection and comparison of the microscopic data obtained for macronucleophagy and PMN, a size difference in their respective cargoes became apparent. PMN vesicles generally appeared to show a larger diameter than their macronucleophagic counterparts, a characteristic that inspired further investigation.

Correlation of target proteins within the presented system was subsequently used for microscopic characterisation of the autophagic machinery in either nucleophagic context.

\subsection{Distinction of the nucleophagic modes}

\subsubsection{Macronucleophagy and PMN cargo sizes differ significantly}

To determine cargo differences between the modes of nucleophagy, two approaches were used for measurements regarding nucleophagic cargo. 
A
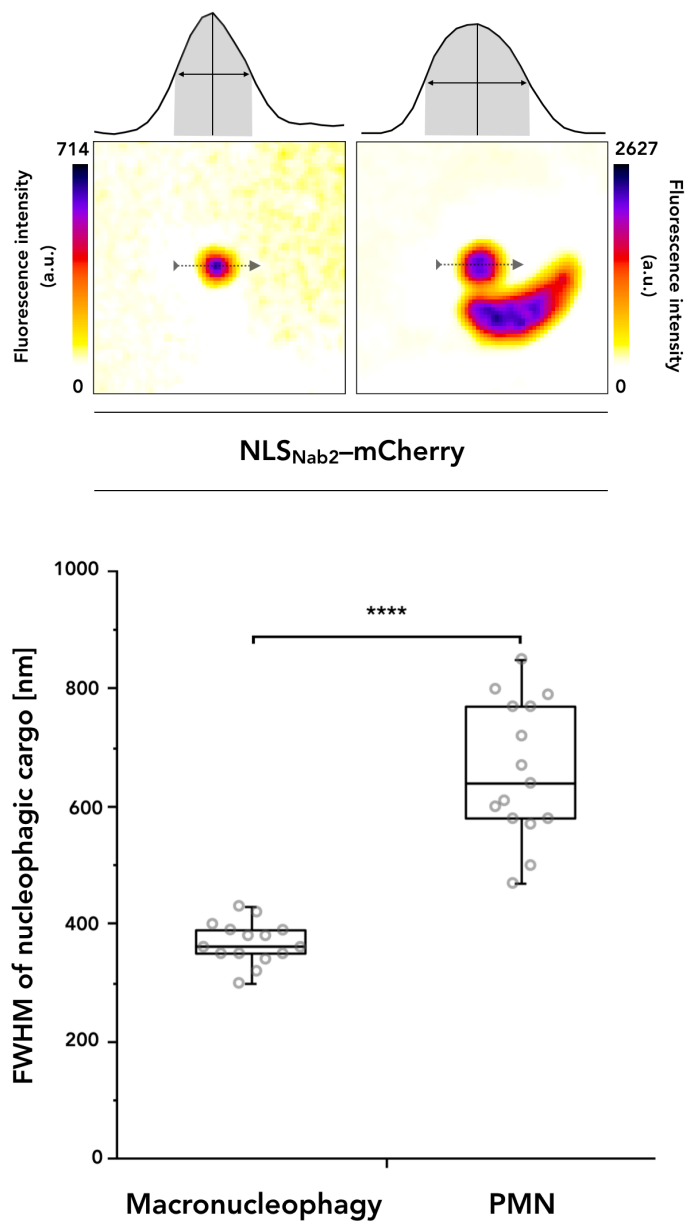
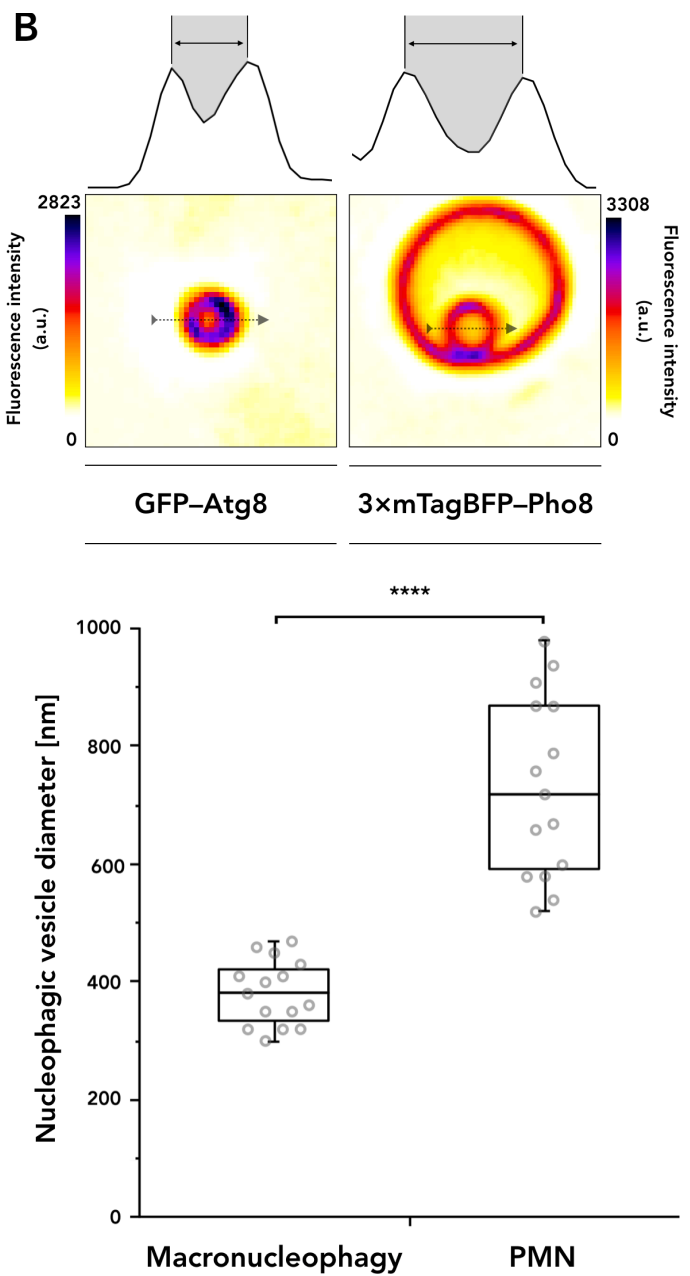

Figure 4.2: Cargo sizes vary between macro- and micronucleophagy

Apparent cargo size differences for the modes of nucleophagy were investigated evaluating widefield fluorescence microscopy images of micro- and macronucleophagic cargo diameter. Datasets are depicted in a box-and-whisker plot. The box is defined as the interquartile range (IQR) containing the median, represented by the line within the box and the whiskers, extending to the last value still within $\times 1.5$ of the IQR. Statistical significance was determined applying an unpaired two-sided t-test, with asterisks representing $\mathrm{P}$-value thresholds: ${ }^{*}=\mathrm{P}<0.05 ;{ }^{* \star}=\mathrm{P}<0.01 ;{ }^{* \star *}=\mathrm{P}<0.001 ;{ }^{* \star \star *}=\mathrm{P}<0.0001$. Experimental evaluations are representative of $n=15$ measurements. (A) Cargo diameters were obtained by determining the full width at half maximum (FWHM) of respective $\mathrm{NLS}_{\mathrm{Nab2} 2}-\mathrm{mCherry}$ signals. The $95 \%$ confidence interval $(95 \% \mathrm{Cl})$ was determined to be 348.3 - $487.7 \mathrm{~nm}$ for macronucleophagy and 596.9 - $725.8 \mathrm{~nm}$ for PMN with an $\mathrm{R}^{2}=0.7569$. (B) Vesicle diameter was determined, quantifying the maximum difference between signal peaks of the outermost vesicular membrane, represented by GFP-Atg 8 and $3 \times$ mTagBFP-Pho8 signals for macronucleophagy and PMN, respectively. The $95 \% \mathrm{Cl}$ was determined to be 351.2-412.8 nm for macronucleophagy and $647.2-818.1 \mathrm{~nm}$ for $P M N$ with an $R^{2}=0.71$. 
Analyses of the $\mathrm{NLS}_{\mathrm{Nab2}}-\mathrm{mCherry}$ signal were performed by obtaining the full width at half maximum (FWHM) of cargo fluorescence intensity (Figure 4.2A). Statistical evaluation revealed highly significant differences between the datasets of the nucleophagic modes, when applying a two tailed t-test. Data collected for cargo analyses is displayed in a box-and-whisker plot. The box plot shows that macronucleophagic cargo with a diameter in an interquartile range (IQR) of 350 $390 \mathrm{~nm}$ can be distinguished easily from PMN cargo with a diameter in an IQR of 580 - $770 \mathrm{~nm}$. With a maximum observed cargo size of $430 \mathrm{~nm}$, for macronucleophagy and a minimum cargo size of $470 \mathrm{~nm}$ observed for PMN, the nucleophagic mode can be determined with a high confidence by analysis of the $\mathrm{NLS}_{\mathrm{Nab2}}-\mathrm{mCherry} \mathrm{cargo} \mathrm{signal.}$

For reference with previously observed sizes of autophagic structures, the diameter of macronucleophagic autophagosomes and PMN vesicles was determined, analysing their outermost surrounding membranes, labelled with GFP-Atg8 and 3×mTagBFP-Pho8, respectively (Figure 4.2B). Complementary to measurements of $\mathrm{NLS}_{\mathrm{Nab2}-\mathrm{mCherry}}$ cargo signals, differences between macronucleophagy and PMN were highly significant for respective vesicle diameter. As opposed to analyses for the $\mathrm{NLS}_{\mathrm{Nab2}}-\mathrm{mCherry}$ signal, vesicle diameters were obtained, determining the max. distance of signal peaks from opposing sides of the circular membrane. A slightly larger diameter was expected in relation to the cargo, which is mostly covered by the obtained experimental data. The IQR for macronucleophagy is $335-420 \mathrm{~nm}$ and $590-870 \mathrm{~nm}$ for PMN. The max. value for macronucleophagic vesicle diameter is $470 \mathrm{~nm}$ while the min. value for $\mathrm{PMN}$ is $520 \mathrm{~nm}$.

Cargo diameter and the ability to resolve GFP-Atg 8 surrounding cargo beyond punctate signals was subsequently used for visualisation of autophagosome biogenesis (Figure 4.4). 


\subsubsection{Identification of suitable cargo for measurements regarding PMN}

In the process of identifying a cargo exclusive for the PMN process, Nvj1, as the core component of NVJ formation, was investigated, relative to nucleus and vacuolar membrane signals.

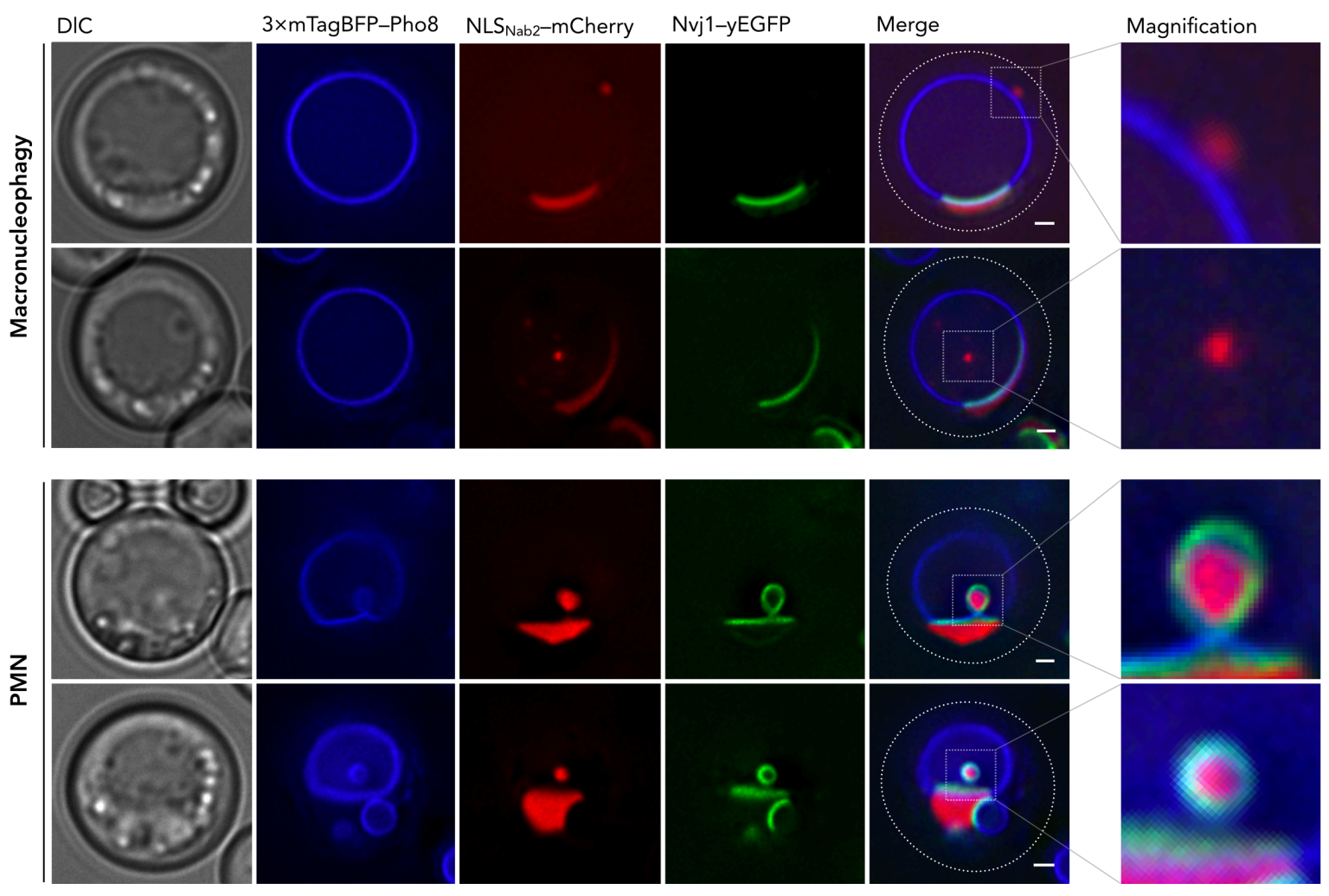

Figure 4.3: Nvj1 is degraded by PMN but not by macronucleophagy

Macronucleophagic- and PMN cargo are depicted in fluorescence microscopy alongside $3 \times$ mTagBFP-Pho8, NLS $\mathrm{Nab2}_{2}-\mathrm{mCherry}$ and respective DIC images. NVJ resident protein Nvj1 fused with yEGFP was expressed under its native promoter to ensure correct localisation and prevent leakage of the protein by overabundance. Merged images detailing nucleophagic cargo with respect to Nvj1-yEGFP are additionally depicted as magnified $(\times 4)$ images. Scale bars represent $1 \mu \mathrm{m}$.

For this purpose, an Nvj1-yEGFP construct was generated, containing the ORF of Nvj1 fused to yEGFP in the low-copy (CEN/ARS) pRS316 backbone. To further avoid overexpression and thereby mislocalisation of Nvj1 (Pan et al., 2000), its native promoter (here defined as 1,000 bp upstream of the Nvj1 ORF) was included for expression, when cloning the C-terminally tagged construct. 
Indeed, microscopic investigation showed that PMN vesicles contained Nvj1yEGFP as part of the NVJ, while macronucleophagic vesicles did not (Figure 4.3). Specifically, neither macronucleophagic cargo located at the vacuolar membrane (Figure 4.3; first row) nor intravacuolar signals (Figure 4.3; second row) colocalised with Nvj1-yEGFP. Vacuolar membrane containing PMN vesicles clearly showed incorporation of Nvj1-yEGFP. As expected, Nvj1-yEGFP localised between vacuolar membrane and nucleoplasmic signals. Its presence within PMN vesicles was also not restricted to the budding process (Figure 4.3; third row), but could be observed for intravacuolar bodies as well (Figure 4.3; fourth row). Intravacuolar bodies were recorded to ensure subsequent degradation of Nvj1-yEGFP.

Nvj1-yEGFP was therefore deemed a reliable cargo for measurements of autophagic degradation, exclusive to PMN and used for further research regarding the micronucleophagic process (Figure 4.15).

\subsection{Microscopic characterisation of macronucleophagy}

Microscopic investigations into macronucleophagy, also termed Atg39-mediated autophagy (Mochida et al., 2015), were focused on localisation of the autophagic machinery in various stages of autophagosome formation, for the fact that the ring shaped autophagosomal membrane could be visualised in detail, using the previously established setup (Figure 4.2A), a process previously shown to be possible only under non-native conditions (Suzuki et al., 2013; Lin et al., 2018; Graef et al., 2013; Sun et al., 2013). 


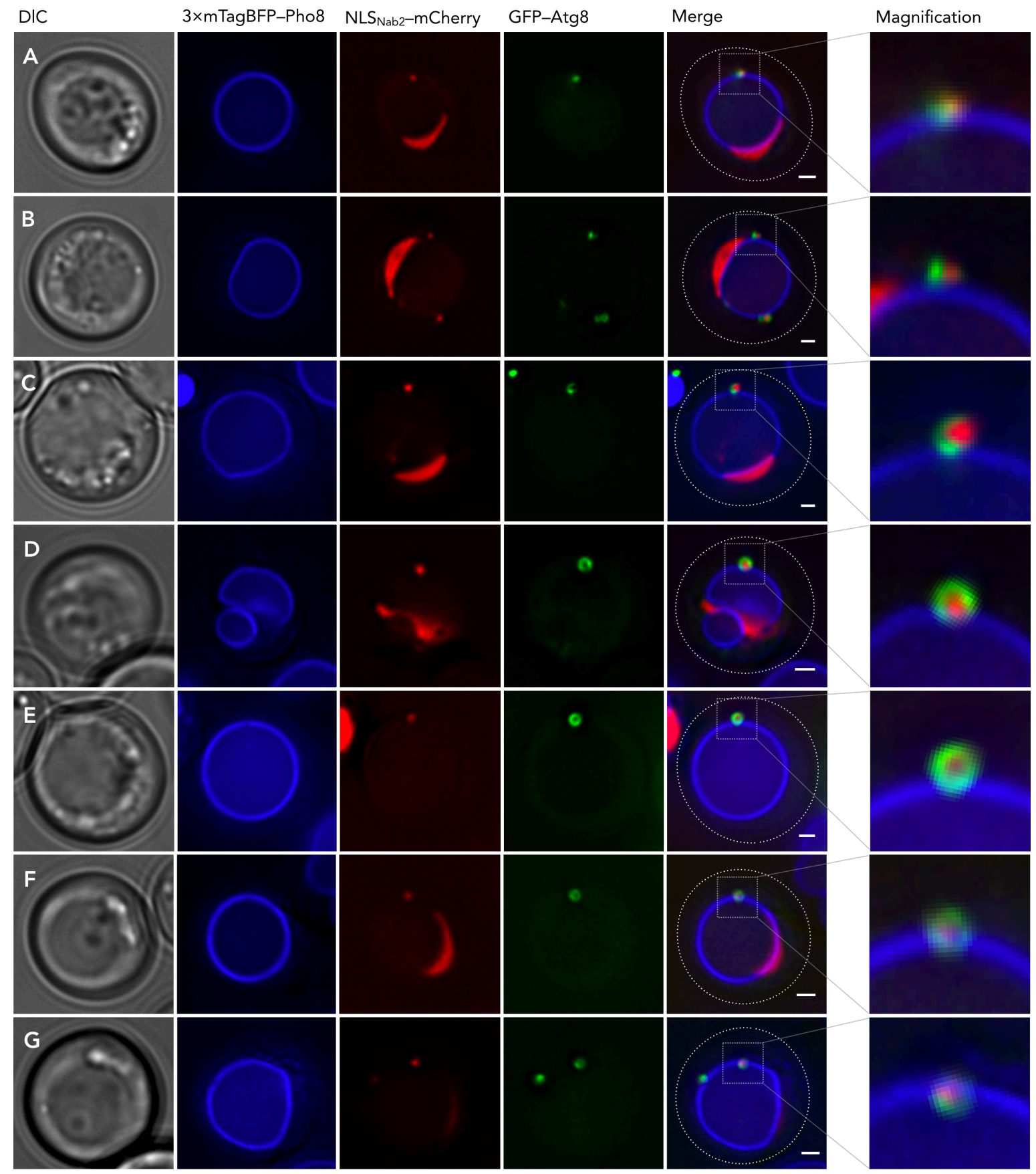

Figure 4.4: Autophagosome biogenesis in macronucleophagic context

The steps of autophagosomal formation were identified in fluorescence microscopy using GFP-Atg8 alongside $3 \times$ mTagBFP-Pho8, NLS $\mathrm{Nab}_{2}-\mathrm{mCherry}$ and respective DIC images. Merged versions of all channels detailing the stages of autophagosome biogenesis are depicted additionally as magnified $(\times 4)$ images. Stages of autophagic membrane expansion, circularisation and fusion are arranged from $(\mathbf{A}-\mathbf{G})$ in the predicted order of their occurrence. Scale bars represent $1 \mu \mathrm{m}$. 


\subsubsection{Formation of autophagosomes in nucleophagic context}

Presence of Atg8 at the PAS, the IM and the autophagosome, and its subsequent delivery in autophagic bodies to the vacuole make it an appropriate marker for visualisation of all stages of the autophagosomal formation process (Kirisako et al., 1999; Suzuki et al., 2001; Suzuki et al., 2013).
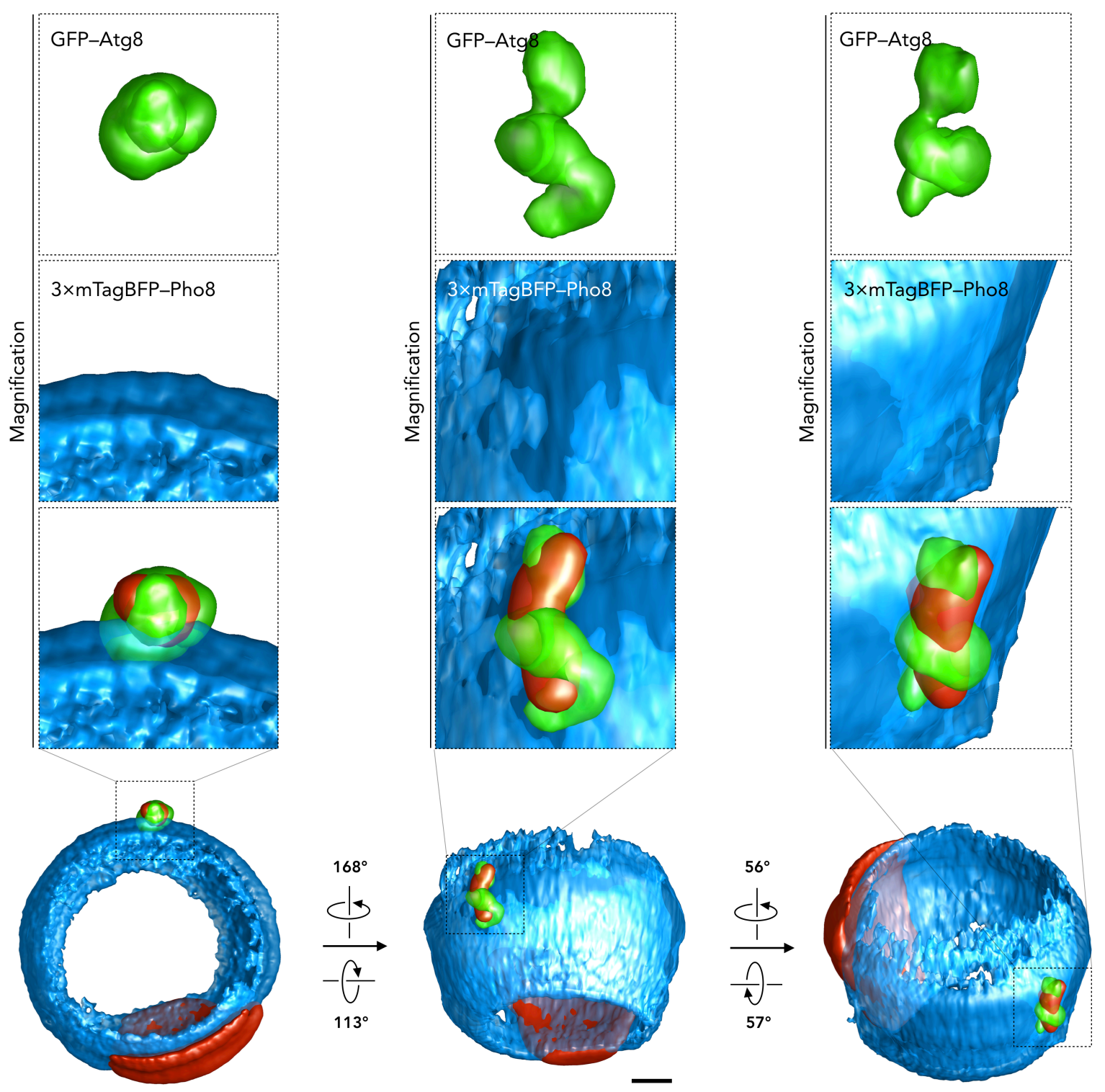

Figure 4.5: 3D representation of a crescent Atg 8 signal

3D projections of a crescent GFP-Atg8 signal in macronucleophagic context alongside $\mathrm{NLS}_{\mathrm{Nab} 2}$-mCherry and $3 \times \mathrm{mTagBFP}-\mathrm{Pho} 8$ were generated from 24 individual z-stacks, each $200 \mathrm{~nm}$ apart. Sites of nucleophagic occurrence are additionally shown in detail as magnified $(\times 3)$ images. The scale bar represents $1 \mu \mathrm{m}$. 
The individual macroautophagic steps were present and visually distinct, when investigating the process of macronucleophagy by observation of GFP-Atg8 (Figure 4.4).

PAS-like, punctate structures of GFP-Atg8 partially overlapped with the NLS $\mathrm{Nab2}_{2}$ mCherry signal, always proximal to the vacuolar membrane (Figure 4.4A). Clearly distinct from punctate signals, various stages of membrane extension representative of the growing IM were localised next to the $\mathrm{NLS}_{\mathrm{Nab2}}-\mathrm{mCherry}$ signal (Figure 4.4B), presumably followed by continuous expansion of the IM into crescent GFP-Atg8 signals and continuous envelopment of nuclear cargo (Figure 4.4C). Autophagosomes, just prior to closure were observed, as well as fully formed autophagosomes (Figure 4.4D \& E). Transfer stages into the vacuole were also observed. Transfer stages were identified as such by observation of GFPAtg8 signals at least partially overlapping with the vacuolar membrane as well as localisation of GFP-Atg8 in the vacuolar lumen (Figure 4.4F \& G).

Projection of the z-stacks obtained for GFP-Atg8 in macronucleophagic context was carried out to display novel insights into the process of autophagosome formation facilitated by observation of macronucleophagy. 3D projections revealed aspects to the formation process, that are masked in both images of single stacks and 2D stack projections. Crescent (Figure 4.6) and circular (Figure 4.7) autophagosomal membrane signals are shown for their visual significance with regard to information gain in 3D. Crescent GFP-Atg8 signal was in 3D shown enveloping nuclear cargo in helical manner, overlapping with the vacuolar membrane signal with each helical revolution (Figure 4.6). Circular GFP-Atg8 signals were shown to extend from the vacuolar membrane as multiple, finger-like extensions enveloping their designated cargo, with their tips reaching towards another (Figure 4.7). 

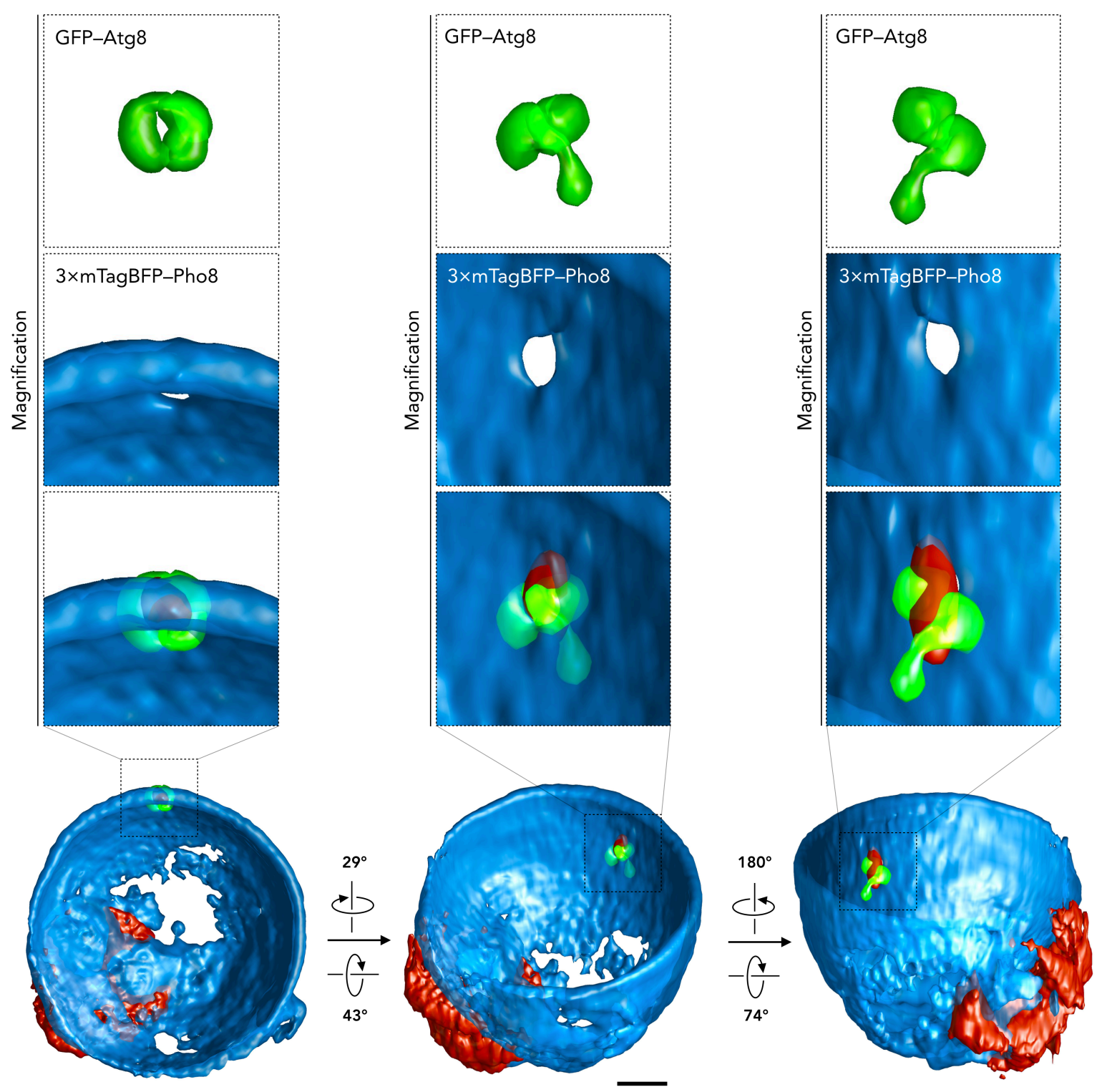

Figure 4.6: 3D representation of a circular Atg8 signal

3D projections of a circular GFP-Atg8 signal in macronucleophagic context alongside $\mathrm{NLS}_{\mathrm{Nab} 2}$-mCherry and $3 \times$ mTagBFP-Pho8 were generated from 24 individual z-stacks, each $200 \mathrm{~nm}$ apart. Sites of nucleophagic occurrence are additionally shown in detail as magnified $(\times 3)$ images. The scale bar represents $1 \mu \mathrm{m}$.

Analysis of fully formed autophagosomes revealed its spherical structure, corresponding to the cargo shape (Figure 4.8). Extending in a joint manner lateral to the vacuolar membrane, the observed stages of autophagy share an ellipsoid appearance of cargo and autophagic machinery. 


\subsubsection{Reduction of Pho8 in the vacuolar membrane coincides with late stages of autophagosome formation}

Observable in 3D depictions and single microscopy stacks, a decrease of $3 \times$ mTagBFP-Pho8 signal proximal to the autophagic machinery was observed for later stages (Figure 4.4E - G) formation of autophagosomes (Figure 4.6; Figure 4.7), prompting further investigation of the vacuolar membrane in the context.
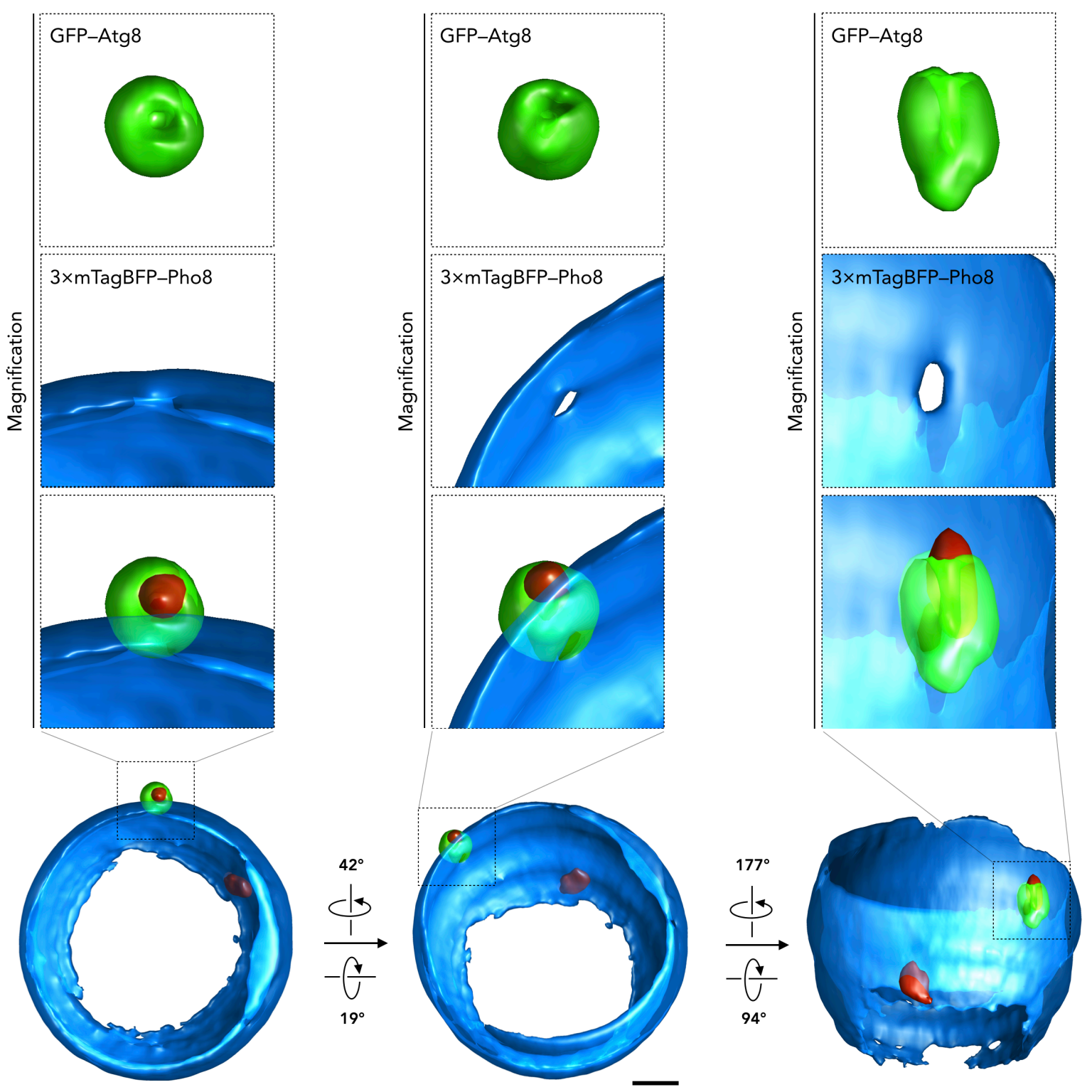

Figure 4.7: 3D representation of the late macronucleophagic process

3D projection of a GFP-Atg8 signal in late stages of the macronucleophagic process alongside NLS $\mathrm{Nab2}_{2}-\mathrm{mCherry}$ and $3 \times \mathrm{mTagBFP}-\mathrm{Pho} 8$ were generated from 24 individual zstacks, each $200 \mathrm{~nm}$ apart. Sites of nucleophagic occurrence are additionally shown in detail as magnified $(\times 3)$ images. The scale bar represents $1 \mu \mathrm{m}$. 
Closer inspection of the respective fluorescence profiles revealed, that a reduction in fluorescence intensity of the $3 \times$ mTagBFP-Pho8 vacuolar membrane signal, coincided with overlaps of late stage, ring shaped GFP-Atg8 signals (Figure 4.8A-C).
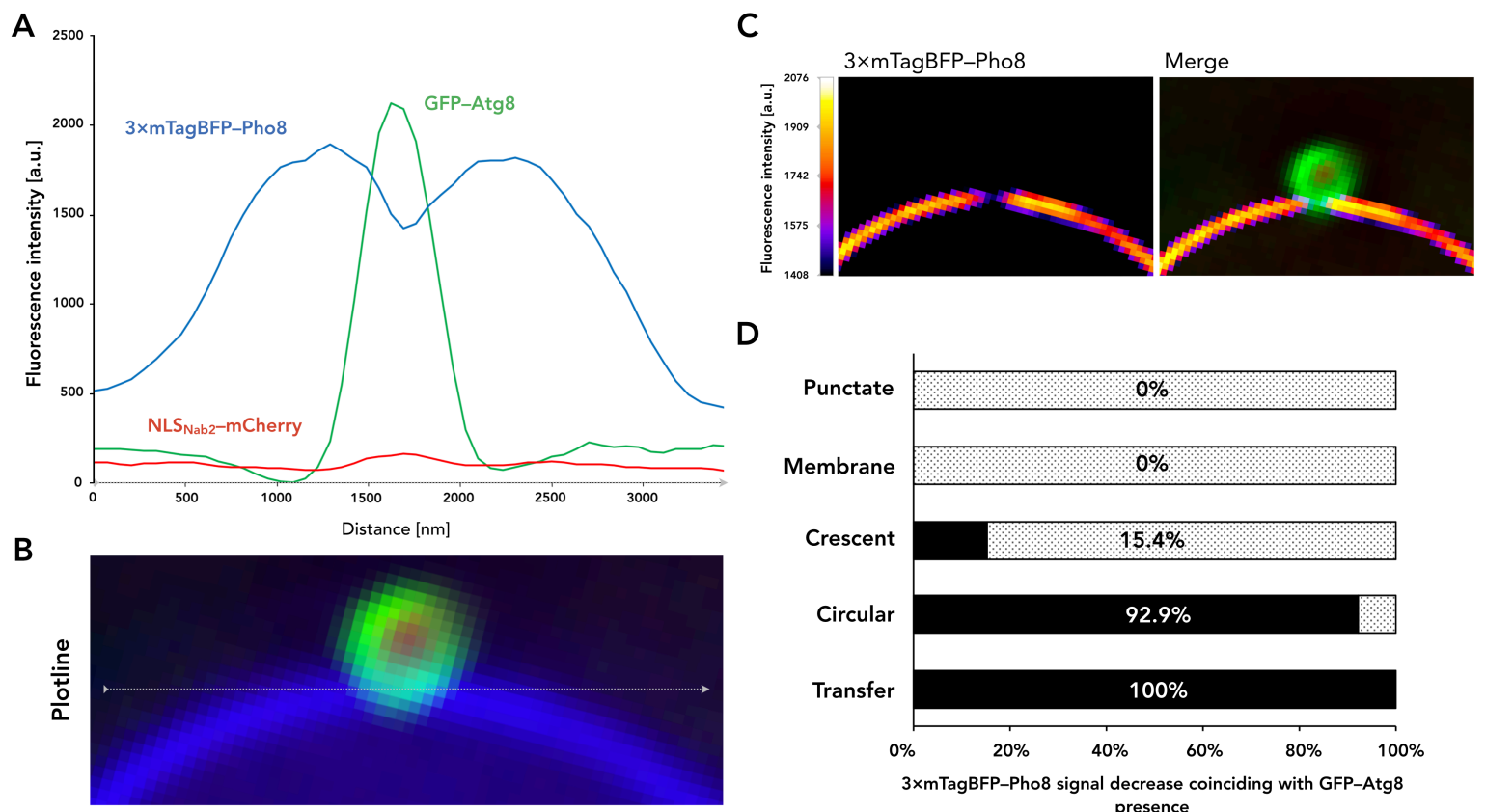

D

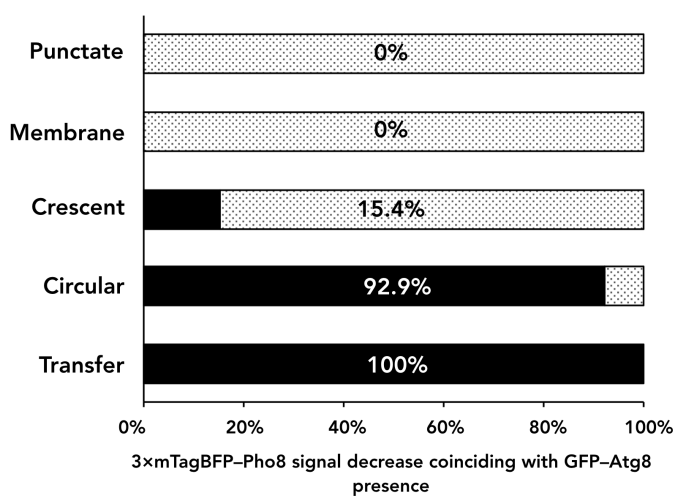

Figure 4.8: Reduced quantities of Pho8 in the vacuolar membrane coincide with autohagosomal vacuolar contact sites in late stages of macronucleophagy

(A) A fluorescence profile for the $3 \times$ mTagBFP-Pho8 signal portion in contact with the circular GFP-Atg8 signal is depicted, with the applied plotline (B), which is representative of the $\mathrm{x}$-axis of the fluorescence profile in $\mathrm{nm}$. The plotted fluorescence profile represents measured fluorescence intensities. (C) Recorded 3×mTagBFP-Pho8 signal intensities are depicted in heatmap fashion, both individually and for reference merged with the GFPAtg8 and $\mathrm{NLS}_{\mathrm{Nab} 2}-\mathrm{mCherry}$ channels. (D) Correlation of $3 \times$ mTagBFP-Pho8 signal decrease coinciding with GFP-Atg8 presence with observed stages of autophagosome biogenesis. Experimental evaluations are representative of $n \geq 10$ measurements.

Importantly, overlaps of autophagosomes and vacuolar membrane frequently demarcated the only portion of reduced $3 \times$ mTagBFP-Pho8 signal found in the vacuolar membrane, or at least a reduction to this extent (Figure 4.6; Figure 4.7). Investigation of 3D reconstructions revealed the singnal reduction to be circular in shape and on approx. $300-500 \mathrm{~nm}$ in diameter. Earlier, non-circular stages of autophagosome formation in contrast, did not coincide with decreased Pho8 
signals (Figure 4.4A - C; Figure 4.5), associating the distinct membrane structure with late stages of autophagosome formation.

\subsubsection{Localisation of the macronucleophagic cargo receptor Atg39}

As the receptor for pnER and thereby the proposed receptor for Atg39-mediated nucleophagy, Atg39-yEGFP was localised within the macronucleophagic system. While Atg39 was previously detected in the pnER and colocalised with Atg8 (Mochida et al., 2015), its precise localisation in macroautophagy was not shown. Detailed localisation of Atg8 upon collection of macronucleophagic data (Figure 4.4) suggested the potential for fine mapping of autophagic components, within the process of autophagosomal formation.

Fluorescence microscopy of the Atg39-yEGFP fusion protein shows a distribution reminiscent of the circular autophagosome pattern observed for GFP-Atg8 and additional signals surrounding the nucleus, reaffirming its general presence in the pnER (Figure 4.9). While Atg39-yEGFP colocalizes with the macronucleophagic NLS $\mathrm{Nab2}_{2}-\mathrm{mCherry}$ signal adjacent to the vacuolar membrane as previously shown (Mochida et al., 2015), detailed investigation reveals it to be surrounding the cargo signal, a localisation in line with its designation as an autophagic cargo receptor. While both cargo and main nucleus (including the NVJ) appeared to be

fully surrounded by Atg39-yEGFP (Figure 4.9B), signals located around nuclear cargo structures were generally stronger than those surrounding the pnER (Figure $4.9 A)$. 


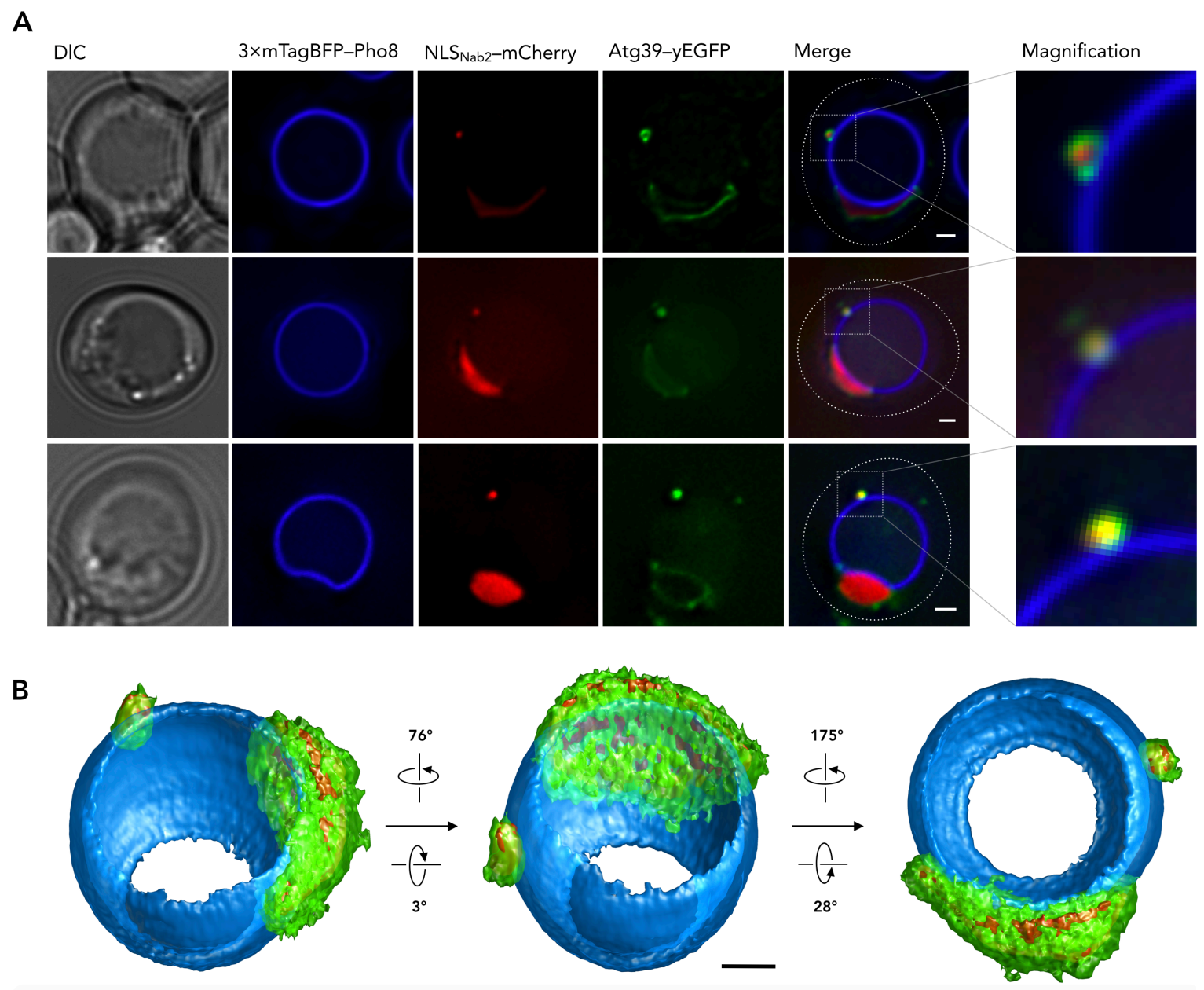

Figure 4.9: Localisation of Atg39 in macronucleophagic context

(A) Cargo receptor Atg39 fused to yEGFP is depicted in fluorescence microscopy alongside $3 \times$ mTagBFP-Pho8, NLS $\mathrm{Nab}_{2}-\mathrm{mCherry}$ and respective DIC images. Merged images detailing macronucleophagic cargo with respect to Atg39-yEGFP are additionally depicted as magnified ( $\times 4$ ) images. (B) A 3D projection generated from 24 individual zstacks, each $200 \mathrm{~nm}$ apart highlighting presence of Atg39-yEGFP surrounding macronucleophagic cargo and main nucleus, including the NVJ. Scale bars represent $1 \mu \mathrm{m}$.

Further investigation confirmed the predicted accumulation of Atg39 around macronucleophagic cargo (Figure 4.10) in relation to the nucleus. Accumulation of the cargo receptor implies a potential prerequisite mechanism depending on Atg39 quantities for potential definition and abstraction of nuclear material from the main nucleus. 
A

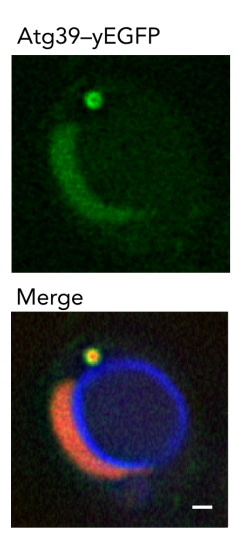

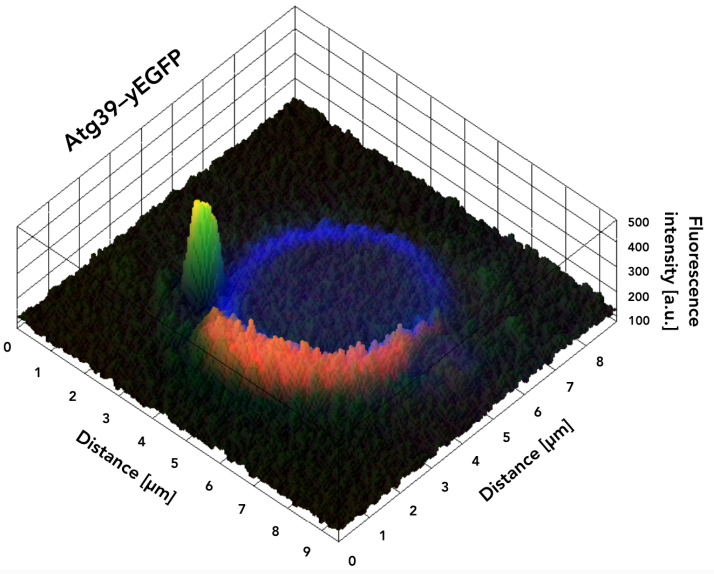

B

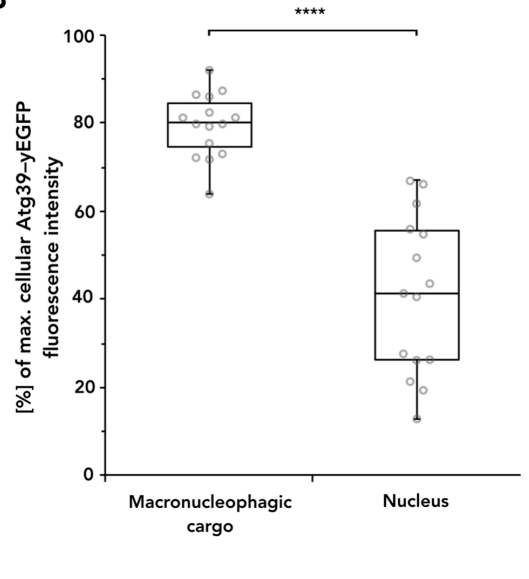

Figure 4.10: Atg39 accumulates around macronucleophagic cargo

(A) Exemplary evaluation of nuclear Atg39-yEGFP signals in comparison with those surrounding macronucleophagic cargo. A 3D surface plot shows the merged image, with signal intensities obtained for Atg39-yEGFP projected onto the image. The scale bar represents $1 \mu \mathrm{m}$. (B) Average Atg39-yEGFP fluorescence intensities surrounding macronucleophagic cargo and the main nucleus were comparatively evaluated, normalising obtained data using max. cellular Atg39-yEGFP fluorescence intensity as a $100 \%$ reference. Collected datasets are depicted in a box-and-whisker plot, with the box defined as the IQR, containing the median represented by the line within the box and the whiskers, extending to the last value still within $\times 1.5$ of the IQR. Statistical significance was determined applying an unpaired two-sided t-test, with asterisks representing $\mathrm{P}$-value thresholds: ${ }^{\star}=\mathrm{P}<0.05 ;{ }^{* \star}=\mathrm{P}<0.01 ;{ }^{\star \star \star}=\mathrm{P}<0.001 ;{ }^{\star \star \star \star}=\mathrm{P}<0.0001$. An $\mathrm{R}^{2}=0.6802$ was determined for the tested dataset. The $95 \% \mathrm{Cl}$ was determined to be $75.6-83.71 \%$ for macronucleophagic cargo signals and $31.04-50.94 \%$ for those surrounding the nucleus. Experimental evaluations are representative of $n=15$ measurements.

\subsubsection{The selective cargo adaptor Atg11 in macronucleophagic context}

Required for recruitment of the autophagic machinery to target receptor complexes, Atg11 is an interactor of the 11-BR containing macronucleophagic cargo receptor Atg39 (Mochida et al., 2015). Investigation of Atg11 localisation within the macronucleophagic system was carried out, aiming to further characterise its functional involvement beyond colocalisation with the autophagic machinery. 


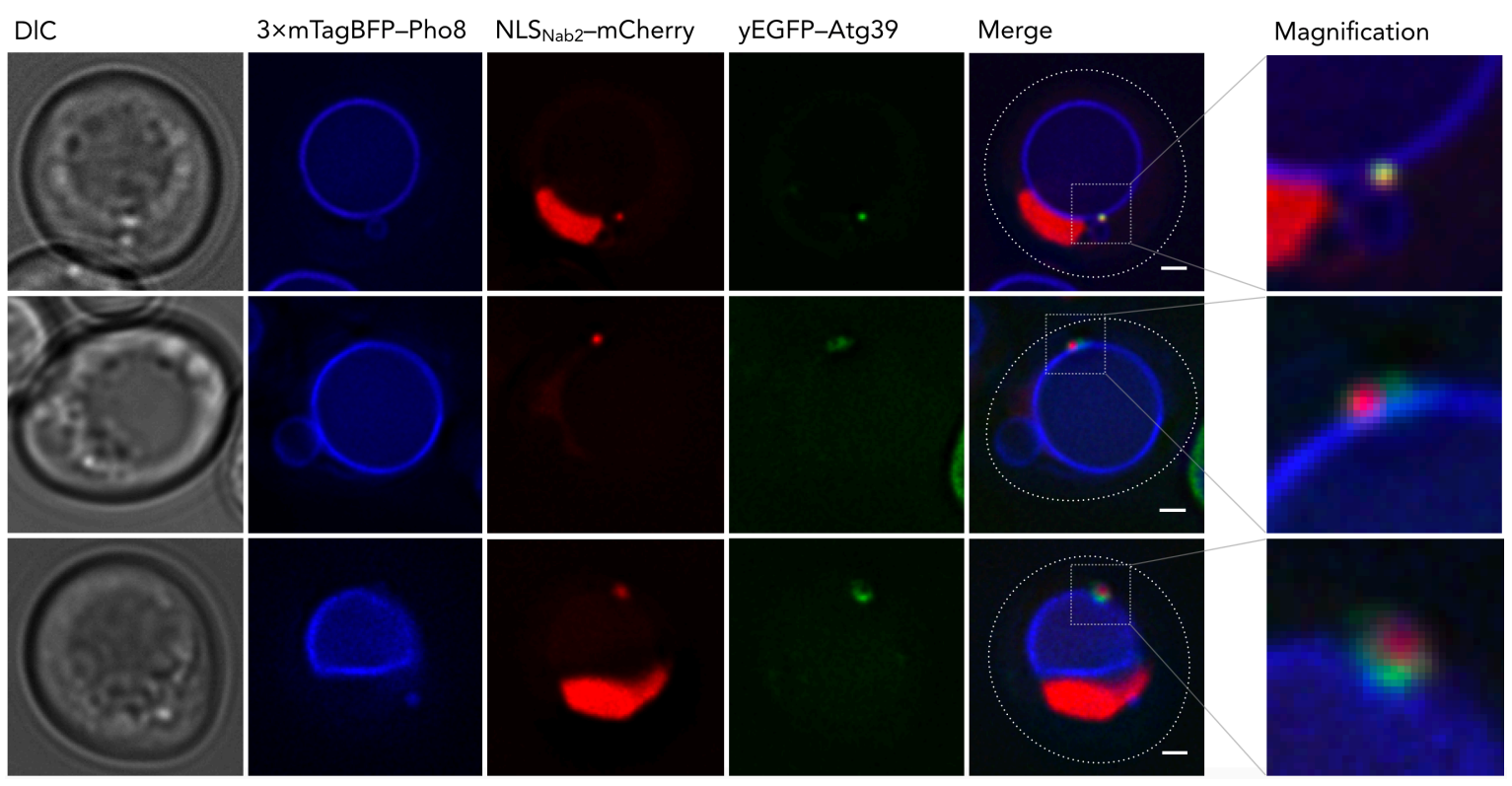

Figure 4.11: Localisation of Atg11 in macronucleophagic context

Atg39 interactor Atg11 fused to yEGFP is depicted in fluorescence microscopy alongside $3 \times$ mTagBFP-Pho8, NLS $\mathrm{Nab2}_{2}-\mathrm{mCherry}$ and respective DIC images. Merged images detailing macronucleophagic cargo with respect to yEGFP-Atg11 are additionally depicted as magnified $(\times 4)$ images. Scale bars represent $1 \mu \mathrm{m}$.

Like Atg39-yEGFP and GFP-Atg8, yEGFP-Atg11 signals were observed in close proximity to $\mathrm{NLS}_{\mathrm{Nab2}}-\mathrm{mCherry}$ cargo signals (Figure 4.11). Lacking pnER localisation observed for Atg39, yEGFP-Atg11 also shows less prominent signals, when compared to GFP-Atg8. Atg11 signals were enriched at contact sites of nuclear cargo with the vacuolar membrane and generally absent at the distal side of the cargo relative to the vacuole.

\subsection{Detailing molecular mechanics of PMN}

\subsubsection{Atg8 is present between the vacuolar sequestering arms}

Analogous to the approach for macronucleophagy, involvement of the autophagic machinery in PMN was investigated by localisation of GFP-Atg8. The essential functionality and continuous presence of Atg8 within the autophagic system in general and its essential functionality for microautophagic processes in particular, made Atg8 a compelling choice for further investigation. 


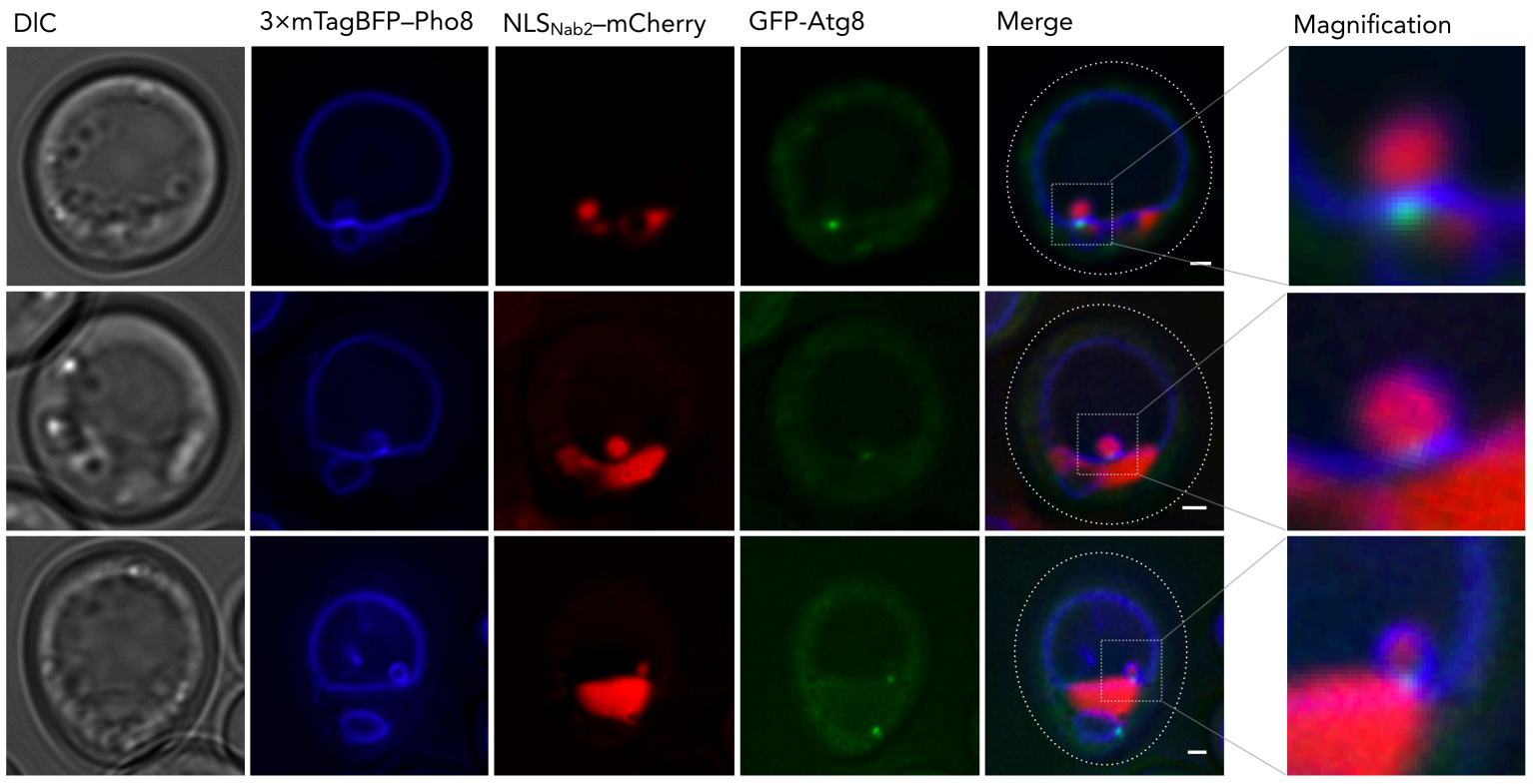

\section{Figure 4.12: Localisation of Atg8 within budding PMN vesicles}

Cargo receptor interactor Atg8 fused to GFP is depicted in fluorescence microscopy alongside $3 \times$ mTagBFP-Pho8, NLS $\mathrm{Nab}_{2}-\mathrm{mCherry}$ and respective DIC images. Merged images detailing the PMN process with respect to GFP-Atg8 are additionally depicted as magnified $(\times 4)$ images. Scale bars represent $1 \mu \mathrm{m}$.

For the first time, the localisation of GFP-Atg8 in context of forming PMN vesicles was revealed. GFP-Atg8 is detected at the encounter site of the vacuolar sequestering arms that separate micronucleus and main nucleus (Figure 4.12). In this context, GFP-Atg8 consistently appears as a punctate structure, sometimes additionally surrounded by a diffuse signal, implying ongoing recruitment of the protein.

The distinct localisation of GFP-Atg8 incited further investigation of the structure with respect to its localisation upon release of the PMN vesicle into the vacuolar lumen. Either continuous localisation between the vacuolar arms or persistence at the NVJ allow for further understanding of autophagic involvement in the PMN process. 


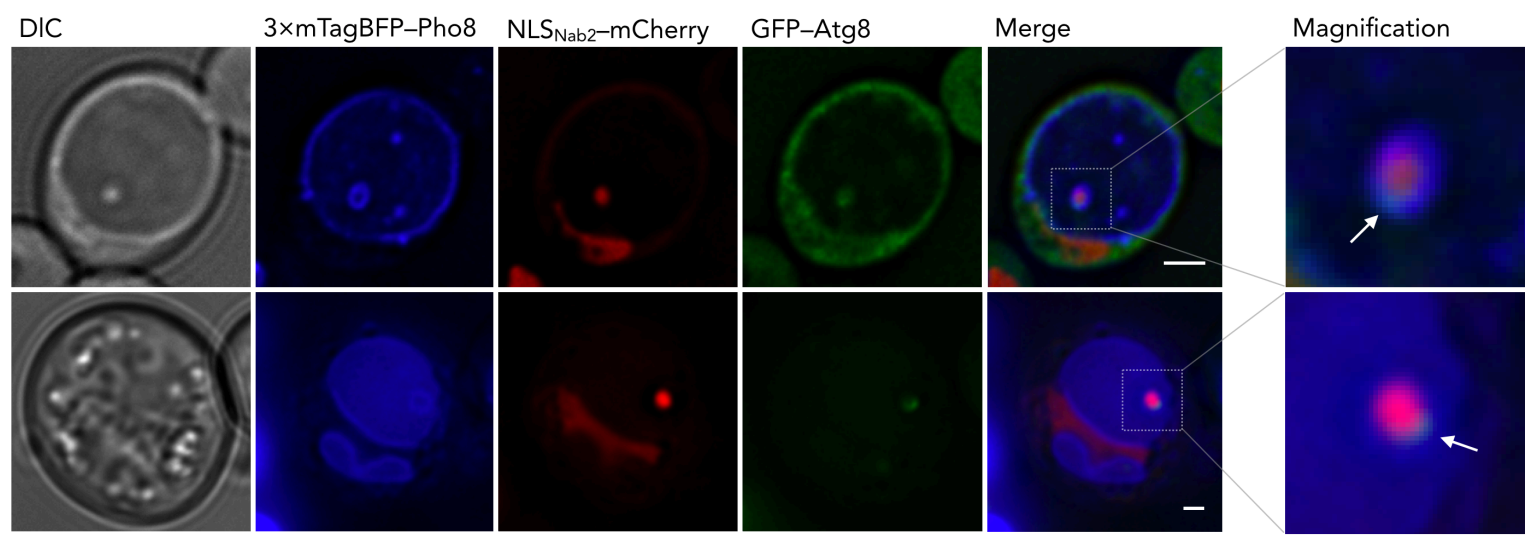

Figure 4.13: Localisation of Atg8 in intravacuolar PMN vesicles

Highlighting its presence in intravacuolar PMN vesicles, Atg8 fused to GFP is depicted in fluorescence microscopy alongside $3 \times$ mTagBFP-Pho8, NLS $\mathrm{Nab2}_{2}-\mathrm{mCherry}$ and respective DIC images. Merged images, with arrows indicating GFP-Atg8 presence at intravacuolar PMN vesicles are additionally depicted as magnified $(\times 4)$ images. Scale bars represent $1 \mu \mathrm{m}$.

To this end, cells were screened for PMN vesicles, that had budded off, but had not yet been degraded within the vacuolar lumen. Continuous localisation of singular, punctate structures of GFP-Atg8 within the vacuolar membrane portion of the vesicle was observed (Figure 4.13), suggesting functional relevance of its continuous presence between the vacuolar arms, even after release of the vesicle.

Previous observations, made in macronucleophagic context, regarding a distinct vacuolar membrane composition prompted investigation in microautophagic context. Sites of overlap with the autophagic machinery (Figure 4.6) represented by a decrease in $3 \times$ mTagBFP-Pho8 signal intensity, suggested a similar approach in micronucleophagic context.

Investigation of the PMN vesicle membrane in widefield fluorescence microscopy suggests absence of Pho8-labelled vacuolar membrane, coinciding with presence of GFP-Atg8 (Figure 4.11), fluorescence intensity measurements were made at the diffraction limit and therefore require further experimental evidence. 
A
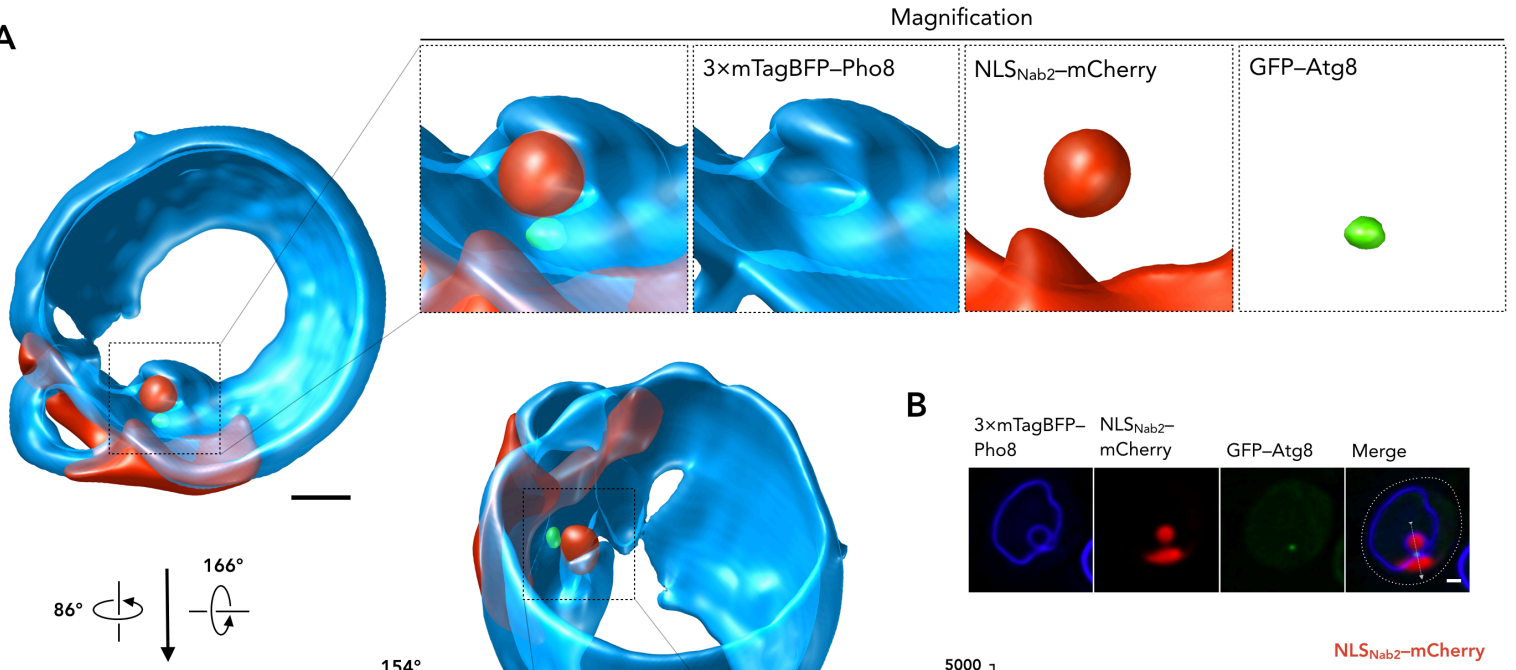

B

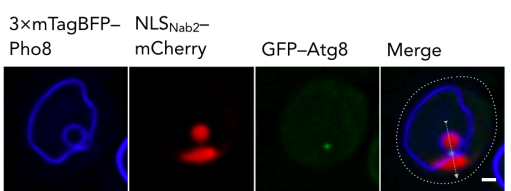

$\left.86^{\circ} \frac{\mathrm{s}}{1}\right|_{\downarrow} ^{166^{\circ}}-\frac{1}{4}$
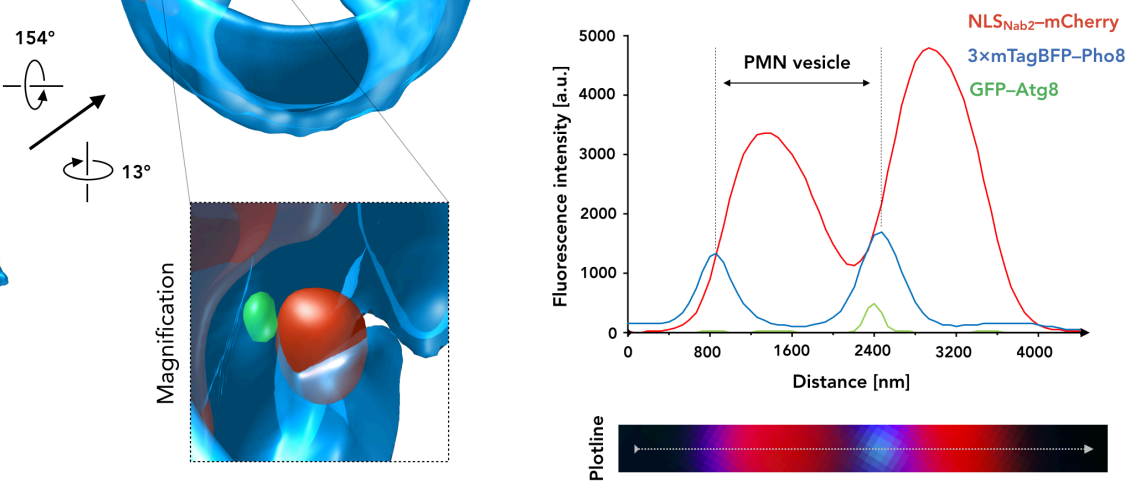

Figure 4.14: No connections remain between PMN vesicle and nucleus

(A) To exclude remaining connections between nucleus and micronucleus upon budding in a PMN event, fluorescent channels of GFP-Atg8, NLS $\mathrm{Nab}_{2}-\mathrm{mCherry}$ and $3 \times \mathrm{mTagBFP}-$ Pho8 were recorded as 24 individual z-stacks, each $200 \mathrm{~nm}$ apart and then projected as $3 D$ images. (B) The fluorescence profile of a bulging PMN vesicle in relation to GFPAtg8. Above the fluorescence profile are the microscopic images used for profiling, with a plotline included in the merge image. A detailed depiction of the plotline is shown below the fluorescence profile, which is representative of its $\mathrm{x}$-axis in $\mathrm{nm}$. Scale bars represent $1 \mu \mathrm{m}$.

Due to the problematic nature of previously observed, tubular connections between micronucleus and nucleus, in PMN (Millen et al., 2009), stacks along the z-axis were taken to exclude remaining exchange between the structures.

3D reconstructions of the obtained z-stacks were generated to highlight the fact, that the observed micronuclei did in fact not show any remaining links to the nucleus (Figure 4.14A) and are not transient morphological abnormalities that retract back into the main nucleus. The fluorescence profile (Figure 4.14B) in 
combination with the $3 \mathrm{D}$ reconstruction highlights the exclusive localisation of GFP-Atg8 between the vacuolar arms of PMN vesicles. Its being otherwise absent within the vacuolar membrane portion implies restriction of the microautophagic machinery to the site of vacuolar membrane encounter.

\subsubsection{STED microscopy of autophagic structures by immuno- labelling with nanobodies}

The restricted disposition of diffraction limited systems prevents resolution of structures beyond approx. $200 \mathrm{~nm}$ (approx. $230 \mathrm{~nm}$ in widefield fluorescence microscopy). Neither punctate accumulations, nor diffuse recruitment signals observed for GFP-Atg8 in PMN context could be resolved further on the DeltaVision ${ }^{\circledR}$ widefield fluorescence microscopy setup.

Application of STED microscopy in combination with a novel labelling protocol developed by Ries et al. (2012) and Kaplan \& Ewers (2015) for yeast cells, enables detection of STED-able dyes. The system implements sdAb-mediated decoration of popular fluorescent tags such as GFP, RFP or TagBFP and their respective derivatives. In order to find a labelling protocol suitable for investigation of a cellular process that involves organellar interaction and is highly dynamic such as PMN, a series of variations of the described protocols were tested. The protocols describe a method that circumvents the necessity for digestion of the yeast cell wall, by permeabilising it instead, with a low detergent concentration. The described permeabilisation creates cavities in the cell wall, large enough for sdAb to enter. The method was applied for labelling of target proteins for subsequent STED microscopy, while efficiently conserving cellular integrity.

Best results with respect to labelling efficiency and organellar conservation were obtained for fixation with very low concentrations $(0.2 \%, v / v)$ of $G A$ and 
prolonged periods of labelling with the sdAb (approx. $90 \mathrm{~min}$ ). Application of paraformaldehyde compromised organellar distribution and was therefore discarded in favour of GA. Application of GA resulted in emergence of autofluorescence between $487 \mathrm{~nm}$ and $559 \mathrm{~nm}$, which efficiently disturbed signal acquisition of GFP labelled constructs. Attempts at quenching GA derived autofluorescence showed best results when using sodium borohydride efficiently restoring detection of GFP. When present, residual autofluorescence was identified as such by reference with the unaffected far-red Atto647N emission of the anti-GFP sdAb.

\subsubsection{Establishing STED microscopy in autophagic context}

Punctate structures of GFP-Atg8, proximal to the vacuole and approx. $300 \mathrm{~nm}$ in diameter, were resolved by STED microscopy (Figure 4.15). Revealed to be either smaller, punctate structures, or extended membrane-like structures, approx. $350 \mathrm{~nm}$ in length and $130 \mathrm{~nm}$ in width, the applied protocol allowed for an increase of resolution while maintaining autophagosomal and vacuolar structure.

Detection of the Atto647N coupled sdAb also showed weaker, punctate signals throughout the cell, which could not be detected for GFP-Atg8. These background signals were not present in Abberior ${ }^{\circledR}$ Star 580 coupled sdAb, but work was continued using Atto647N coupled sdAb for its emission in the far-red spectrum, which in contrast to Abberior ${ }^{\circledR}$ Star 580 could easily be distinguished on both microscopy setups, from the emission spectrum of mCherry. Known to bind membranes unspecifically, due to its lipophilic character and low solubility in water, Atto647N could be replaced for novel dyes with similar excitation and emission spectra such as the Abberior ${ }^{\circledR}$ Star $635 \mathrm{P}$ which shows significantly reduced background (Wurm et al., 2012). In this work, background signals of Atto647N were efficiently reduced by increased periods of blocking with NGS 
and three successive washing steps following completion of the sdAb labelling procedure.

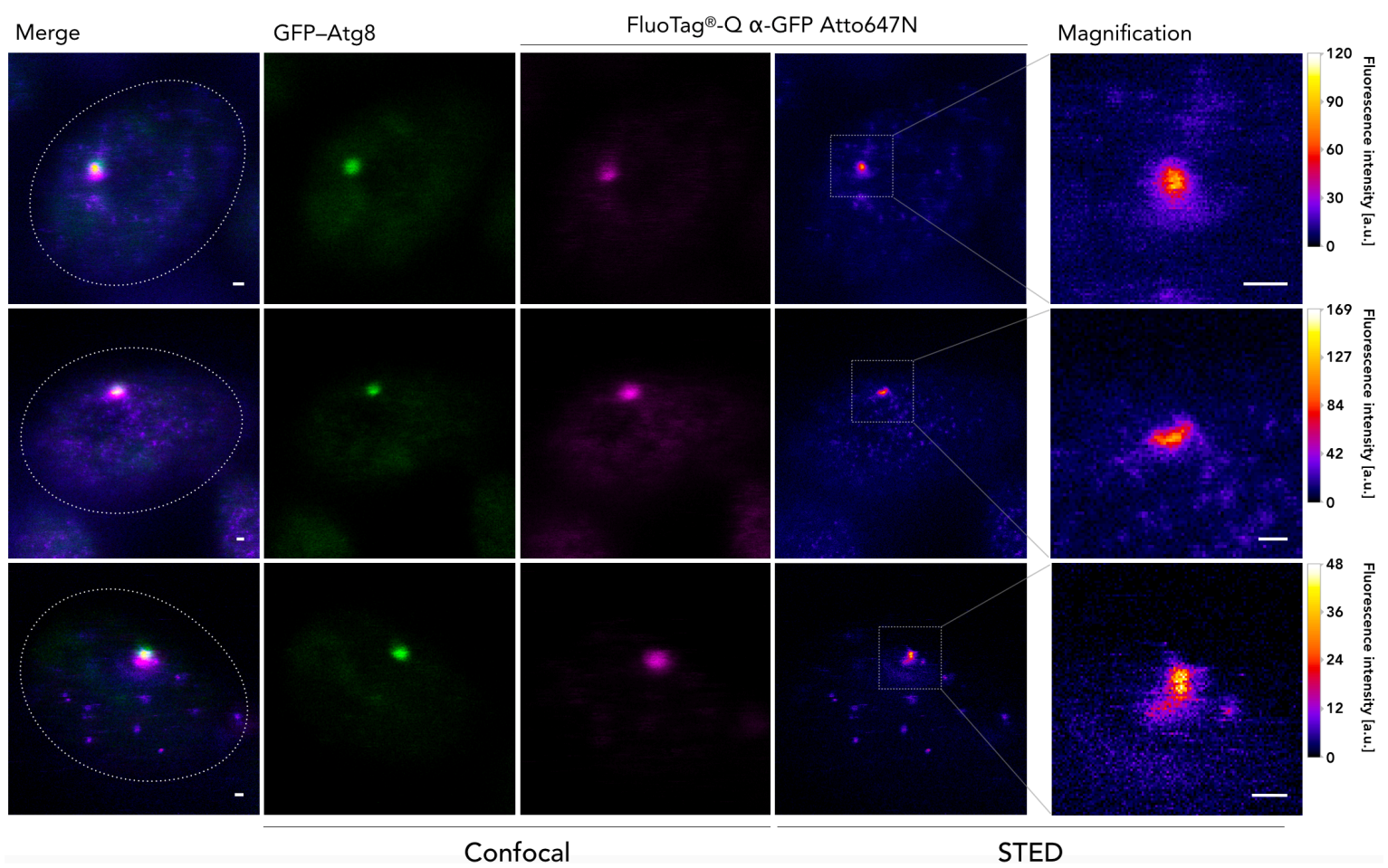

Figure 4.15: STED microscopy of fixed and permeabilised yeast cells

GFP-Atg8 punctae were investigated in an atg $1 \triangle$ strain facilitated by labelling GFP-Atg8 with single domain antibodies (sdAb). STED-able anti-GFP sdAb (FluoTag ${ }^{\circledR}-\mathrm{O} \alpha-$-GFP Atto647N) and recorded applying confocal and subsequent STED microscopy. Merged versions are displayed for labelling reference and cellular outlines were inserted as a dashed line. Magnified $(\times 4)$ images of GFP-Atg8 punctate structures subjected to STED microscopy are included for reference of resolution gain. STED microscopy images are depicted in heatmap fashion. Scale bars represent $0.2 \mu \mathrm{m}$.

\subsubsection{STED microscopy of Atg8 in PMN context}

Reference with cargo sizes analysed for macro- and micronucleophagy (Figure 4.2) allowed for identification of $P M N$ associated micronuclei in confocal and STED microscopy. With $580 \mathrm{~nm}$ of FWHM for the Nab2 ${ }_{\mathrm{NLS}}-\mathrm{mCherry}$ signal of the data depicted in Figure 4.16, cargo is well within the range of cargo values, expected for PMN (Figure 4.2A) and also clearly distinct from values obtained for macronucleophagy. STED microscopy was performed in fixed myo1 $\Delta$ cells. Previously shown to accumulate a higher number of blebs budding into the 
nucleus (Roberts et al., 2003), the strain was used to increase frequency of cells with PMN structures.

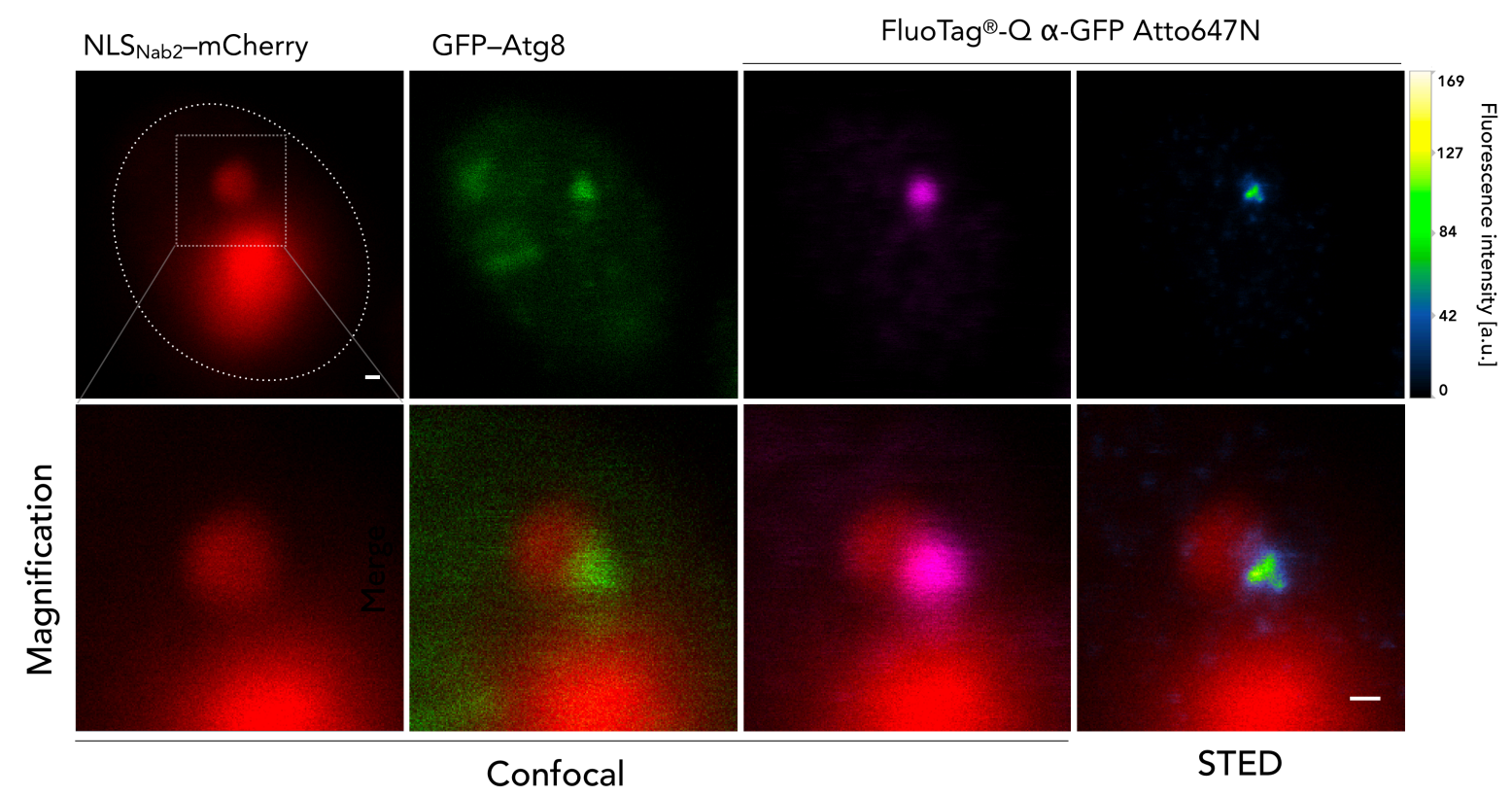

Figure 4.16: STED microscopy reveals a microautophagic membrane structure

PMN situations in myo1 $\Delta$ cells, depicted by visualising nucleoplasm with $\mathrm{NLS}_{\mathrm{Nab}^{-}}$ mCherry alongside GFP-Atg8, additionally labelled with the FluoTag ${ }^{\circledR}-\mathrm{Q} \alpha$-GFP Atto647N were recorded in confocal microscopy. Atto647N was additionally subjected to STED microscopy. All recorded channels were merged with confocal and STED microscopy images of $\mathrm{NLS}_{\mathrm{Nab} 2}-\mathrm{mCherry}$ and the separate Atg8 channels. Scale bars represent $0.2 \mu \mathrm{m}$.

STED microscopy revealed punctate GFP-Atg8 signals detected between the vacuolar arms to be a small (approx. $200 \mathrm{~nm}$ long and $70 \mathrm{~nm}$ wide), membranelike structure seemingly shaped around the micronucleus with a small additional arm of approx. $90 \mathrm{~nm}$ pointing in opposite direction to the micronucleus (Figure 4.16).

\subsubsection{The macroautophagic cargo receptor Atg39 is involved in PMN}

As the cargo receptor for macronucleophagy, Atg39 was also considered an exclusive cargo of macronucleophagy (Mochida et al., 2015; Mostofa et al., 2018). 
Heightened presence in formation of autophagosomes, surrounding nuclear cargo, and additional signals present in the pnER prompted investigations towards a potential role for Atg39 in PMN context.

Fluorescence microscopy of Atg39-yEGFP in a PMN context revealed a localisation similar to GFP-Atg8, with strong punctate accumulation between the vacuolar sequestering arms and as previously observed, additional signals surrounding both nucleus and micronucleus (Figure 4.17). Microscopic data depicts Atg39 as a cargo of PMN. While not as prominent in its presence surrounding macronucleotophagic cargo (Figure 4.9; Figure 4.10), Atg39 clearly surrounds PMN cargo, rendering measurements regarding autophagic turnover of Atg39 unsuitable for distinction of macronucleophagy and PMN.

A

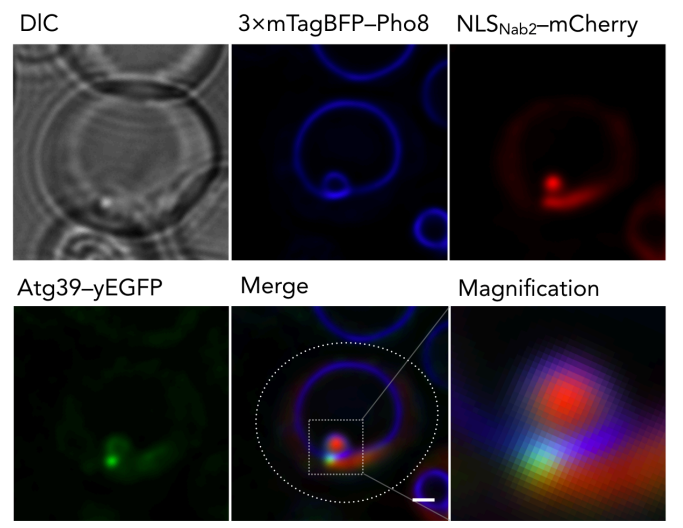

B

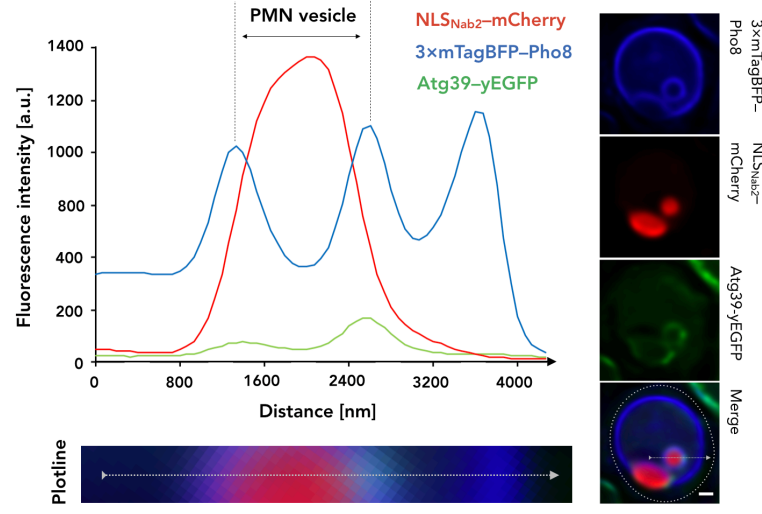

Figure 4.17: Localisation of Atg39 within budding PMN vesicles

(A) Atg39-yEGFP is depicted alongside $3 \times$ mTagBFP-Pho8 visualising the vacuolar membrane and $\mathrm{NLS}_{\mathrm{Nab2}}-\mathrm{mCherry}$ labelling the cell nucleoplasm next to a DIC image and merged versions of all channels with and without the DIC image. Merged images of the micronucleus are depicted in detail as a magnified zoom image. (B) The fluorescence profile of a PMN vesicle in relation to Atg39-yEGFP. To the right of the fluorescence profile are the microscopic images used for profiling, with a plotline included in the merge image. A detailed depiction of the plotline is shown below the fluorescence profile, which is representative of its $\mathrm{x}$-axis in $\mathrm{nm}$. Scale bars represent $1 \mu \mathrm{m}$.

Investigation of autophagic turnover by $\mathrm{PMN}$ requires a cargo component exclusive for the micronucleophagic process to avoid obscuring of the data by 
alternative degradation processes. Nucleoplasm $\left(\mathrm{NLS}_{\mathrm{Nab2}}\right)$, nucleolar components (Nop1) and pnER (Hmg1) were shown to be targeted by both PMN and macronucleophagy alike, making exclusive, quantitative assays particularly difficult to establish (Roberts et al., 2003; Krick et al., 2008; Figure 4.1; Dawaliby \& Mayer, 2010; Mochida et al., 2015; Mostofa et al., 2018). With an essential role in PMN, the NVJ is thought to be exclusively degraded by the PMN process and its core component Nvj1 is not included in macronucleophagic cargo (Figure 4.3). Expression of Nvj1-yEGFP was therefore used to determine PMN-dependent degradation by quantification of cellular Nvj1-yEGFP versus free yEGFP levels under induction of autophagy by nitrogen starvation (Figure 4.18).

Autophagy rates were determined, applying an assay that utilises the relatively resistant structure of GFP and its derivatives toward vacuolar proteases (MeilingWesse et al., 2002). Signal intensities were determined using Fiji and evaluated by calculating the ratio of free yEGFP divided by values measured for Nvj1yEGFP. Values for the wt, $10 \mathrm{~h}$ after induction, were set to $100 \%$ and referenced with an $\operatorname{atg} 1 \Delta$ strain, selected for the essential role of Atg1 in early assembly of the autophagic machinery, and atg39 $\Delta$ to determine involvement of the cargo receptor in PMN.

In line with expectations, proteolytic degradation of Nvj1-yEGFP was barely detectable in the $\operatorname{atg} 1 \Delta$ strain, when compared to the wt. A similar situation was observed for atg39 $\mathrm{\Delta}$. Both $6 \mathrm{~h}$ and $10 \mathrm{~h}$ after starvation in SD-N, degradation of Nvj1-yEGFP was reduced. A significant reduction was observed after $10 \mathrm{~h}$ of starvation, when referenced with the wt strain. Requirement for Atg39 in PMN expands its role as a cargo receptor by the micronucleophagic process, supporting a central function for the protein in overall nucleophagy. 


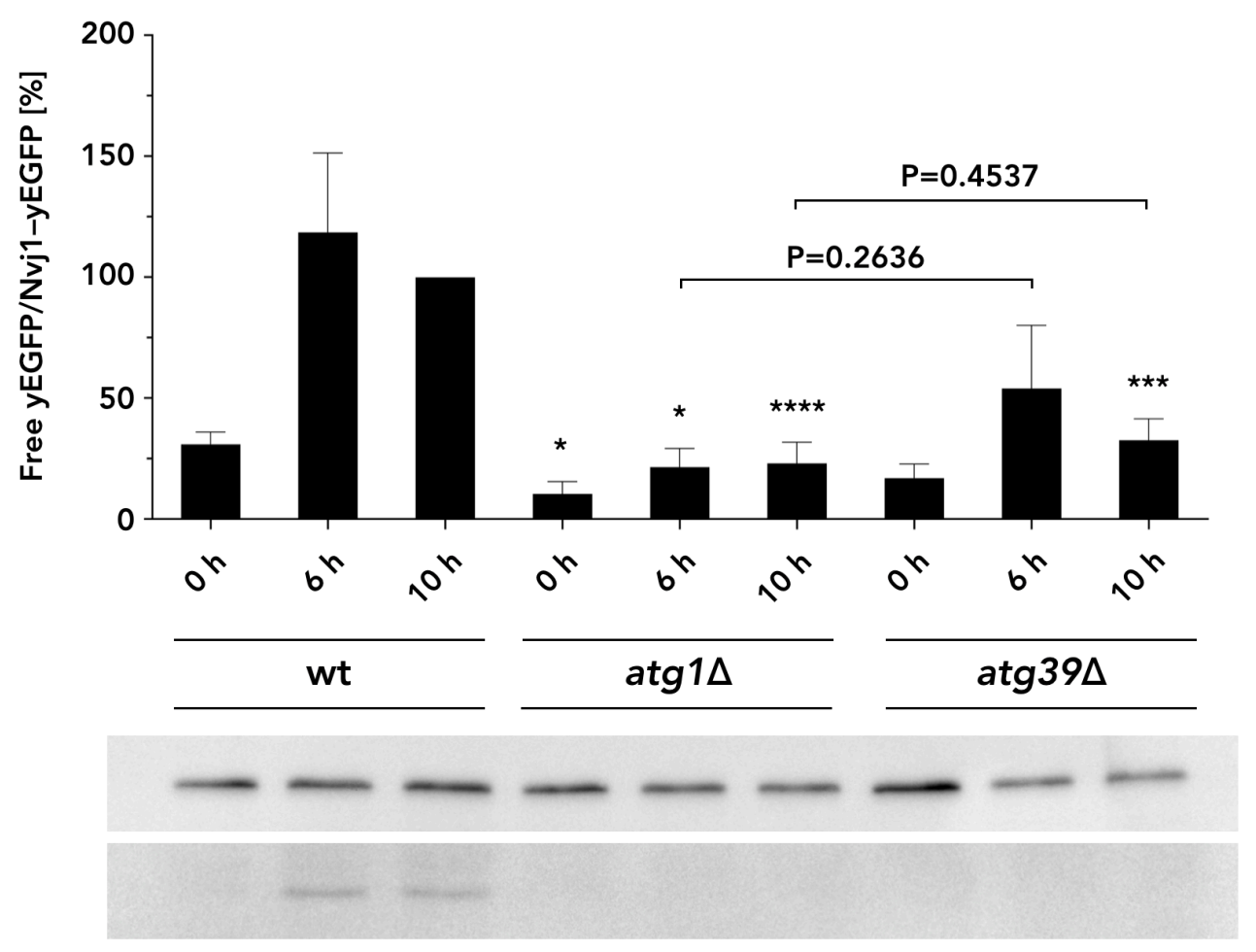

Nvj1-yEGFP

Free yEGFP

Figure 4.18: Atg39 is involved in PMN-dependent Nvj1 degradation

Expression of Nvj1-yEGFP under its native promoter was performed in atg39 $\mathrm{A}$ cells, referenced by the respective wildtype (wt) and an atg1 $1 \triangle$ representative for PMN deficiency. Degradation of native Nvj1-yEGFP quantities was shown to closely represent PMN-exclusive degradation. Upon conclusion of PMN, Nvj1-yEGFP is degraded, but relatively protease resistant, free yEGFP remains in the vacuole and is quantifiable in relation to the Nvj1-yEGFP fusion protein. Whole cell extracts were analysed $0 \mathrm{~h}, 6 \mathrm{~h}$ and $10 \mathrm{~h}$ after exposure to starvation conditions. Signal intensities are depicted in relation to the $10 \mathrm{~h}$ wt value, which was set to represent 100\%. Statistical significances were determined applying a two-sided t-test using Prism 7.0d. Asterisks represent $p$-values: ${ }^{*}=P<0.05 ;{ }^{* \star}=P<0.01 ;{ }^{* \star *}=P<0.001 ;{ }^{* \star \star *}=P<0.0001$. The experimental evaluation is representative of $n=8$ individual experiments. Error bars depict the standard error of the mean.

\subsubsection{Cargo adaptor Atg11 in nucleophagic context}

As a cargo receptor adapter, Atg11 is associated with selective capability of autophagic modes. Requirement for Atg11 in nucleophagy was previously shown (Krick et al., 2008). To test whether the Atg39-Atg11 interaction, observed for macronucleophagy, is preserved in PMN, yEGFP-Atg11 was investigated in PMN context. 
Atg11 appears to localise at the interaction site of the vacuolar arms and microautophagic membrane (Figure 4.19). Investigation of microautophagic vesicles revealed two significant signal peaks within the circular structure within a single z-stack. Three dimensional investigation implies two signals and hints at a possibly ring shaped formation at the tips of the vacuolar sequestering extension. The signals imply an interaction site between Atg11 and Atg39 which appears present within the microautophagic membrane potentially closing the vacuolar extensions.

A DIC

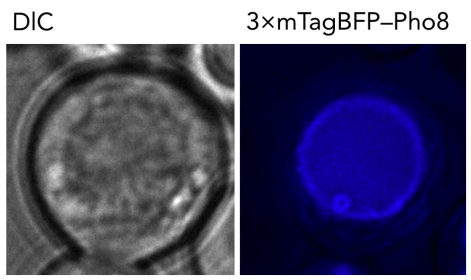

B

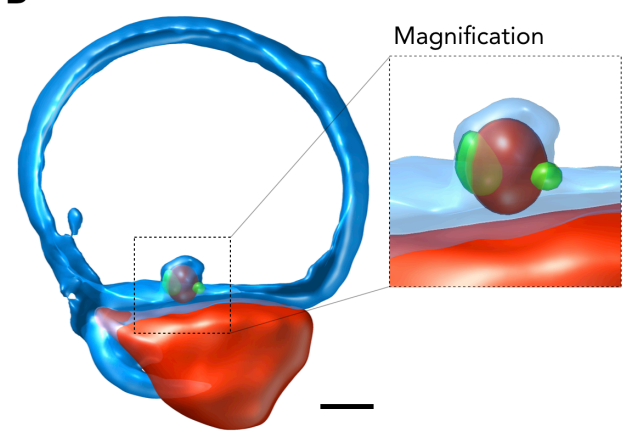

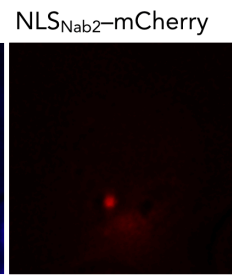
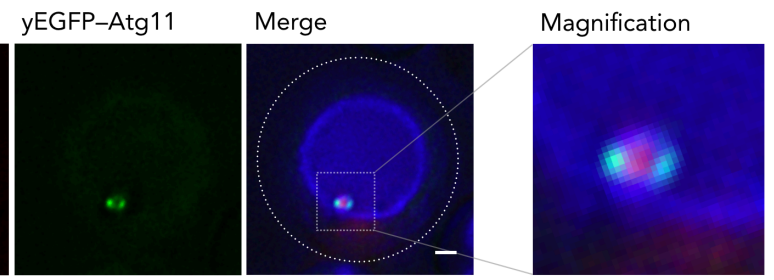

C

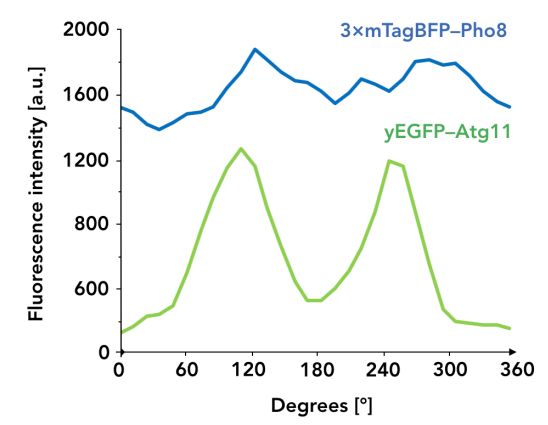

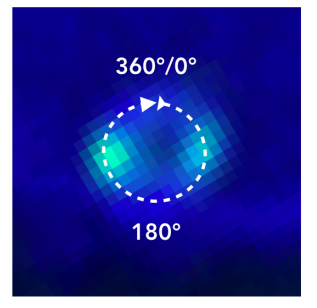

Plotline

Figure 4.19: Localisation of Atg11 within budding PMN vesicles

(A) Atg39 interactor Atg11 fused to yEGFP is depicted in fluorescence microscopy alongside $3 \times$ mTagBFP-Pho8, NLS $\mathrm{Nab} 2_{2}-\mathrm{mCherry}$ and a respective DIC image. A merged image detailing PMN cargo with respect to yEGFP-Atg11 is additionally depicted as magnified $(\times 4)$ images. (B) A 3D projection generated from 24 individual z-stacks, each $200 \mathrm{~nm}$ apart highlighting presence of Atg11 at two distinct sites within the vacuolar membrane bulge (C) A fluorescence profile for the shown PMN vesicle in relation to yEGFP-Atg11. To the right of the fluorescence profile is a detailed depiction of the plotline, which is representative of its $x$-axis in degrees. Scale bars represent $1 \mu \mathrm{m}$.

\subsubsection{Investigation of PI3P distribution in PMN context}

Discovery of an Atg8 containing membrane in the PMN process led to further investigations into the membrane composition with particular regard to the IM of 
the macroautophagic machinery. As an essential component of the autophagic membrane, PI3P is present in all stages of formation including autophagic bodies and its accumulation is required for autophagosome formation and potentially involved in maturation (Kihara et al., 2001; Cheng et al., 2014). Involvement of PI3P was investigated using the Fab1, YOTB, Vac1 and EEA1 (FYVE) PI3P binding domain of EEA1 (FYVEEEA1) fused to GFP (Burd \& Emr, 1998).

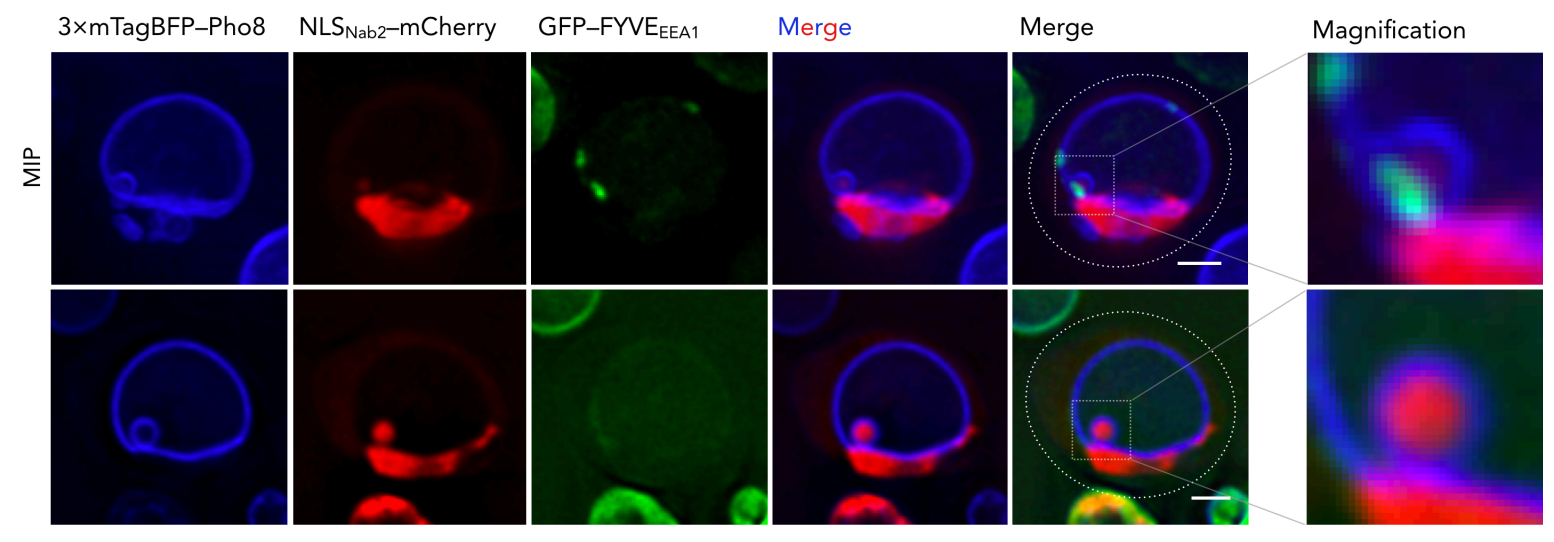

Figure 4.20: Localisation of PI3P within budding PMN vesicles

GFP-FYVEEEA1, recognising PI3P in the cell is depicted in fluorescence microscopy alongside $3 \times$ mTagBFP-Pho8 and NLS $\mathrm{Nab2}_{2}$ mCherry. The upper series shows a maximum intensity projection (MIP) of a PMN vesicle localised at the edge of the NVJ, while the lower series shows channels of a stack of a vesicle within the NVJ. Merged images of $3 \times$ mTagBFP-Pho8 and $\mathrm{NLS}_{\mathrm{Nab2} 2}$-mCherry are additionally shown alongside merged images of all channels detailing macronucleophagic cargo with respect to GFP-FYVE Additionally magnified $(\times 4)$ images are shown. Scale bars represent $1 \mu \mathrm{m}$.

Specific binding of PI3P was shown to occur proximal to PMN vesicles at the vacuolar membrane portion next to the budding micronucleus (Figure 4.20; upper series). Signals did not appear to integrate into the vesicle itself (Figure 4.20; lower series) and could generally not be detected within the NVJ. PI3P might have a potential capacity for initial recruitment of autophagic components to the microautophagic vesicle, lacking a role as an actual constituent of the PMN process. Since GFP-FYVE an alternative explanation to PI3P absence might be a hindrance in accessibility for the GFP-FYVEEEA1 construct. Close association of the junction might confine GFP-FYVE $E_{E E A 1}$ to the non-junction portion of the vacuolar membrane. 


\subsubsection{Involvement of motorproteins in early stages of PMN}

Involvement of motor proteins, previously shown for the autophagic process in general (Ravikumar et al., 2005; Kimura et al., 2008) and PMN in particular (Roberts et al., 2003), prompted investigation by F-actin staining with a phalloidin conjugate.

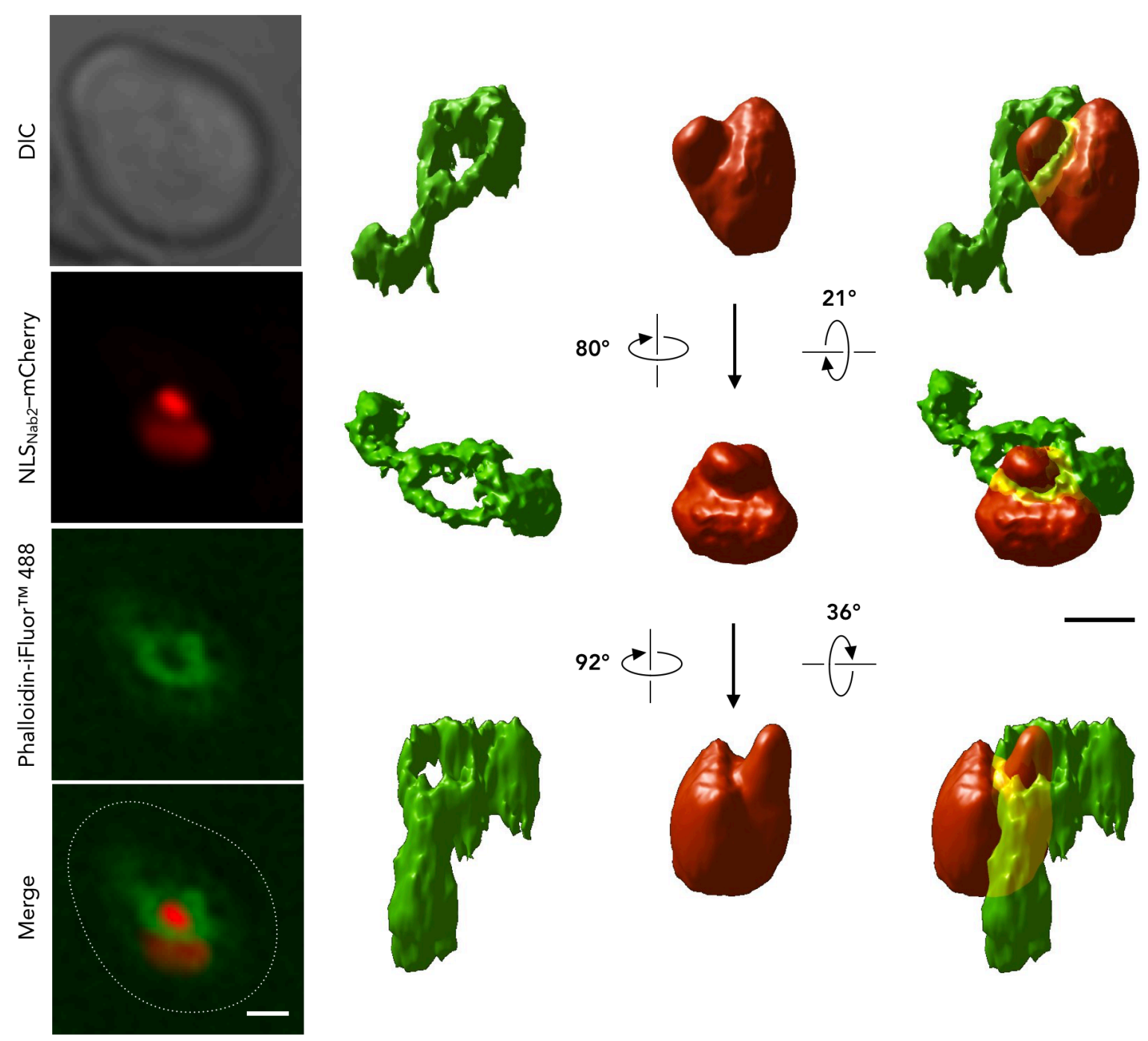

Figure 4.21: Visualisation of F-actin in relation to nuclear bulging

For visualisation of $\mathrm{F}$ - actin, Phalloidin-iFluor ${ }^{\mathrm{TM}} 488$ depicted in fluorescence microscopy alongside $\mathrm{NLS}_{\mathrm{Nab} 2}-\mathrm{mCherry}$ and a respective DIC image. A merged image detailing a nuclear bulge with respect to Phalloidin-iFluor TM 488 is additionally depicted. A 3D projection of the data was generated from 24 individual z-stacks, each $200 \mathrm{~nm}$ apart highlighting ring shaped structure around a nuclear bulge. Scale bars represent $1 \mu \mathrm{m}$. 
Increased bulging of PMN-like structures in myo1 $1 \Delta$ cells described by Roberts et al. (2003) was also utilised in this work, increasing PMN occurrence for STED microscopy (Figure 4.16). Visualisation of F-actin was realised using PhalloidiniFluor $^{\text {TM }}$ 488. Performed after fixation of yeast cells observations showed, what appears to be a ring-like structure, confining a nuclear bulge, reminiscent of a contractile ring (Figure 4.21). Three dimensional visualisation further supported observations of a ring-like structure, shaping around a nuclear bleb and also revealed two opposing poles, extending from the ring towards the cell periphery.

Data collected for F-actin staining supports motorprotein involvement in the PMN process, and suggests early involvement, potentially mediating bulging of the nucleus prior to budding and formation of the microautophagic membrane, elaborating further on the relationship between autophagic machinery and motorproteins.

\subsubsection{PMN presents a regulatory function for lipid metabolism}

The unique composition of the NVJ prompted speculation regarding its potential designation as a Lo domain. Formation of Lo domains depends on the accumulation of cholesterol and coincides with an absence of Vph1 (Toulmay \& Prinz, 2013). The amount of verified NVJ resident proteins associated with lipid metabolism is conspicuously high and strongly infers functional relevance. Presence of proteins capable of interchanging sterols between opposing membranes, present in the NVJ are represented by Lam6/Ltc1 and Osh1 supporting a potential accumulation of sterols in the NVJ. A function for redistribution of sterols and potentially formation and distribution of vacuolar $L_{O}$ domains from the NVJ would in turn, associate PMN with regulation of the process. 

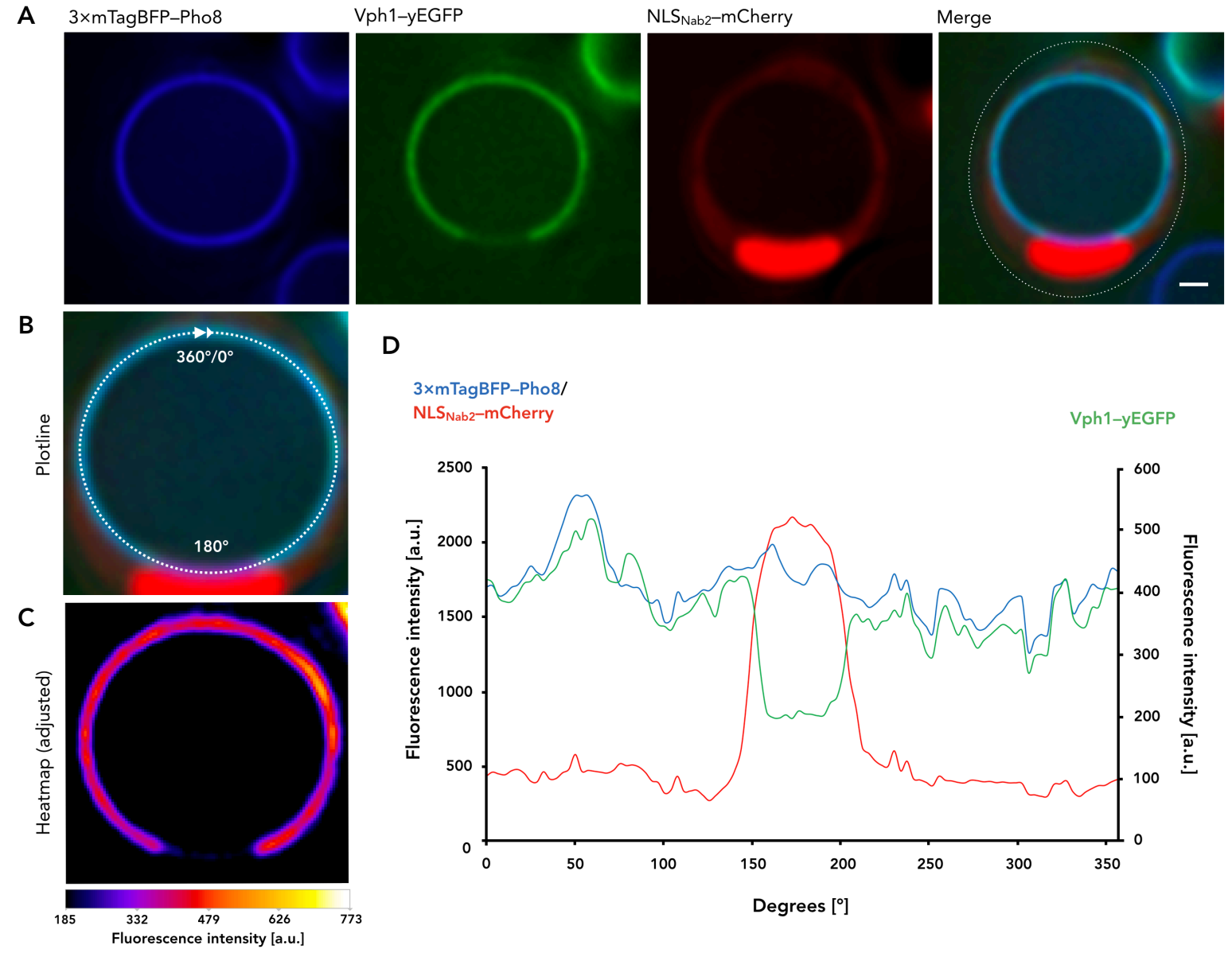

\section{Figure 4.22: Absence of Vph1 from the NVJ suggests a sterol rich domain}

(A) The subunit a of the vacuolar ATPase Vph1 fused to yEGFP is depicted alongside $3 \times$ mTagBFP-Pho8, NLS Nab2 $_{2}$ mCherry and a merged version of all channels. (B) The plotline used to obtain the fluorescence profile is shown superimposed on a merged image, with the indicated degrees corresponding to the fluorescence profile. (C) The Vph1-yEGFP signal was additionally depicted in heatmap fashion alongside the respective fluorescent intensities. (D) The fluorescence profile for all recorded channels depicting the obtained fluorescence intensities with $3 \times$ mTagBFP-Pho8 and NLS $\mathrm{Nab}_{2}-$ mCherry on the left and Vph1-yEGFP on the right $x$-axis plotted against the degrees of the circular plotline alongside the vacuolar membane. The scale bar represents $1 \mu \mathrm{m}$.

Vph1-GFP was expressed to contrast its absence with presence of sterols in the $\mathrm{NVJ}$, and localise the vacuole in relation to the $\mathrm{NLS}_{\mathrm{Nab2} 2}-\mathrm{mCherry}$ labeled nucleus (Figure 4.22). Indeed absence of Vph1, highlighted by both the heatmap depiction and the fluorescence profile for the circular vacuole signal applied to all recorded channels, coincides with the entirety of the vacuole nucleus contact site. The data supports designation of the NVJ as a sterol rich $L_{0}$ domain and affirms previous data (Dawaliby \& Mayer, 2010). 


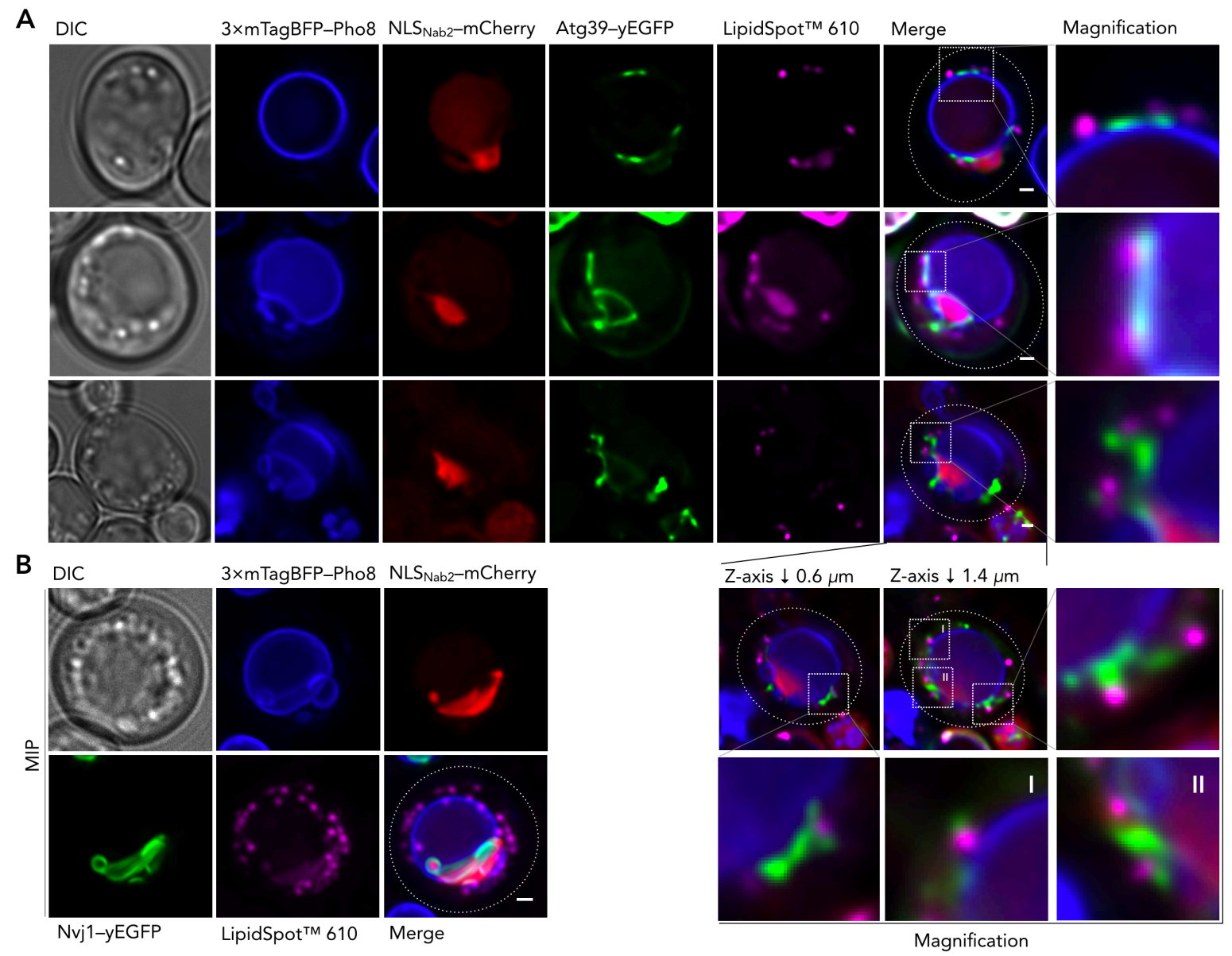

Figure 4.23: Lipid droplet synthesis coincides with PMN and redistribution of Atg39

(A) Cargo receptor Atg39 fused to yEGFP is depicted in fluorescence microscopy in cells under diauxic shift conditions, alongside $3 \times$ mTagBFP-Pho8, NLS $\mathrm{Nab}_{2}-\mathrm{mCherry}$, LipidSpot ${ }^{\mathrm{TM}} 610$ and respective DIC images. Merged images of LD presence alongside Atg39-yEGFP are detailed as magnified ( $\times 3$ first row; $\times 4$ all others) images. For the cell in the third row additional z-stacks are displayed to highlight formation of an Atg39 network. (B) Depicting LD presence alongside PMN occurrence in an MIP of 24 z-stacks, NVJ component Nvj1 fused to yEGFP is depicted in fluorescence microscopy in cells under diauxic shift conditions, alongside $3 \times$ mTagBFP-Pho8, NLS $\mathrm{Nab}_{2}-\mathrm{mCherry}$, LipidSpot $^{\text {TM }} 610$ and a respective DIC image. Scale bars represent $1 \mu \mathrm{m}$.

Implications of Atg39 in microlipophagy (Vevea et al., 2015), as well as LD accumulation at the edge of the NVJ, together with data localising various members of the lipid machinery within, motivated further investigation into changes in overall neutral lipid composition upon disruption of PMN components and Atg39 localisation with respect to LDs. Induction of LD synthesis was achieved by cell cultivation towards diauxic shift conditions (Hariri et al., 2018). LDs were visualised by application of LipidSpot ${ }^{T M}$ 610, 30 min prior to imaging. 
Accumulation of LD was shown to coincide with PMN and a relocalisation of Atg39 (Figure 4.23). Observations of PMN structures after LD accumulation (Figure 4.23B) hint at a potential regulation of the process by degradation of the junction, potentially succeeded by microlipophagy. Redistribution of Atg39 which often connects the pnER to LDs in a meshwork enveloping the vacuole (Figure 4.23A) could either hint at a reshaping event of pnER coinciding with LD synthesis, or a direct association of LDs by Atg39.

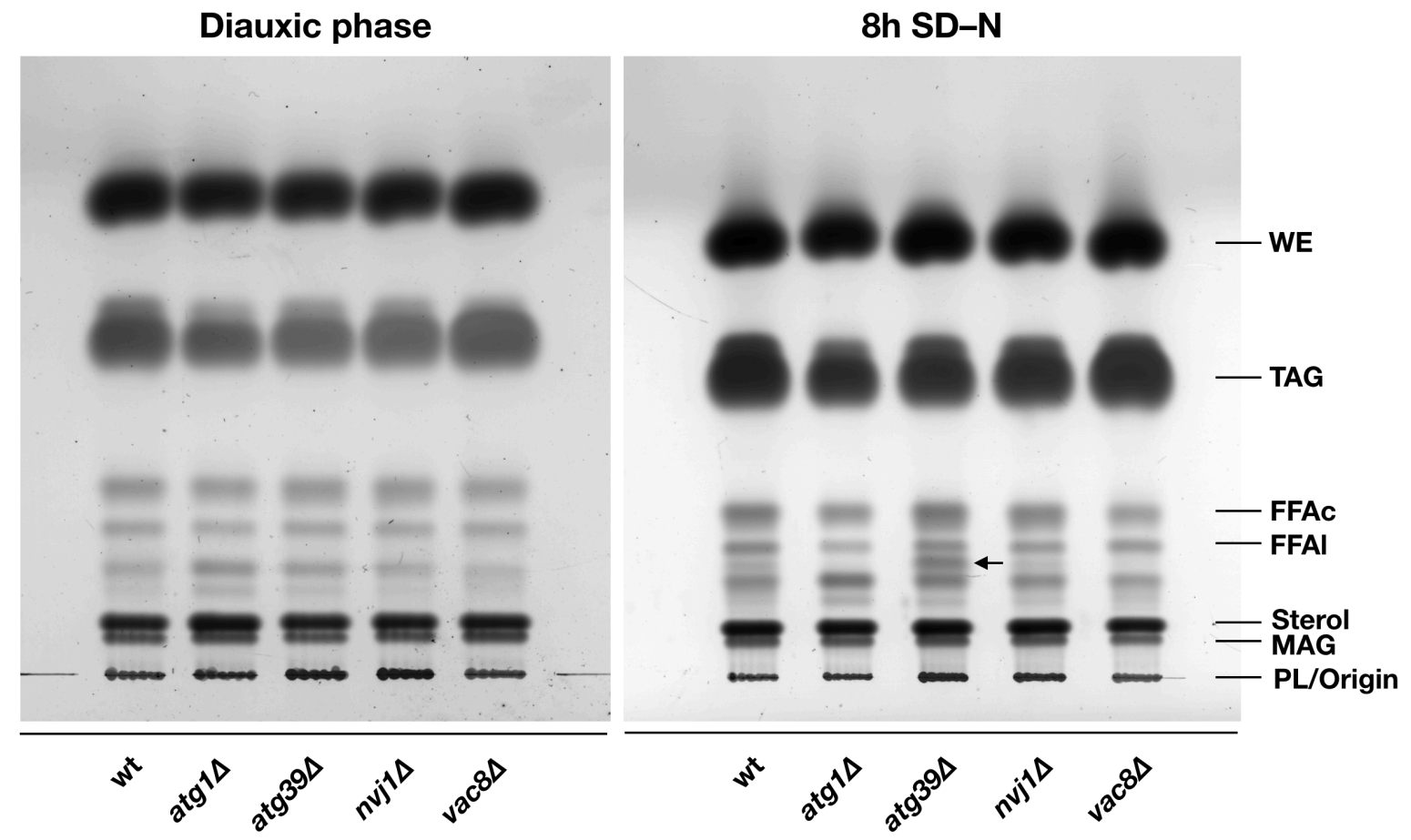

Figure 4.24: Neutral lipid extracts from PMN-disruption strains

Whole cell lipid extracts were spotted on silica coated glass plates and subjected to thin layer chromatography (TLC). Predicted lipid types are indicated alongside the used strains involved in PMN. Lipids were extracted from cells in diauxic phase and $8 \mathrm{~h}$ after incubation in SD-N. Wax esters (WEs), triacylglycerols (TAGs), Free fatty acids (FFAc), Free fatty alcohols (FFAl), monoacylglycerols (MAGs), phospholipids (PLs) were identified by reference.

Whole lipid extracts were obtained from wt and PMN associated mutant strains and subsequently analysed by TLC (Figure 4.24). Identification of bands was performed using standard solutions and additional reference with observations from literature (Heilmann et al., 2012). Wax esters (WEs), TAGs, free fatty acids 
(FFAcs), free fatty alcohols (FFAls), sterols and monoacylglycerols (MAGs) could be identified as such by reference.

While in diauxic phase, neutral lipid extracts did not show strong differences, after incubation for $8 \mathrm{~h}$ in SD-N the atg39 strain showed accumulation of an unknown lipid population (Figure 4.24; arrow indicator). Although the band was present in the wt and the other PMN disruption strains, it was exclusively enriched in the atg39 strain. Further research toward identifying the unknown band and investigations aiming to determine more nuanced differences between the lipid populations have to be carried out to elucidate the specific roles for the NVJ, PMN and Atg39 in lipid metabolism. 


\section{Discussion}

As a mechanism for regulating cellular homeostasis, autophagy targets a variety of cellular components to the vacuole/lysosome. Exercising both selective and unselective functionality, cargo for autophagy includes proteins, aggregates, organelles and also invading pathogens. Two principal modes of autophagy have been described, macroautophagy and microautophagy. Macroautophagy leads to enclosure of cargo in de novo formed, double-membrane vesicles, the autophagosomes, which fuse with the vacuole after fully enveloping its target. In contrast, microautophagy does not require formation of autophagosomes but instead abstracts cargo by invagination of cargo into the vacuolar membrane. Both modes were shown to require a select subset of autophagic proteins, which was thereby defined as the core autophagic machinery.

Nucleophagy was shown to function in both macro- and microautophagic manner. Targeting nuclear material without concluding in cellular death, nucleophagy requires a tightly regulated and highly coordinated underlying mechanism that ensures conservation of the cell genome and its expression machinery.

Macroautophagy of the nucleus was shown to function by recognition of the pnER by means of the pnER resident protein and autophagy cargo receptor Atg39. In addition to both inner and outer NE, portions of the nucleolus were shown to be degraded by Atg39-mediated nucleophagy. Macronucleophagy is considered to be a selective process for the ability of Atg39 to recruit the autophagic machinery by interaction with both Atg8 and Atg11. In this context, Atg39 maintains all characteristics commonly found in autophagic cargo receptors. 
Micronucleophagy targets part of the nucleus in contact with the vacuole by a mechanism described as PMN. PMN functions by envelopment of a specialised MCS, the NVJ. PMN thereby results in degradation of multiple membranes, specifically the vacuolar membrane, the INM and ONM, but also a portion of the nucleoplasm. Like macronucleophagy, PMN was shown to also digest nucleolar material.

Aside from the similarities in cargo composition both nucleophagic modes depend on the core autophagic machinery, with requirement for a selective subset of the Atg proteins. Determination of specific cargo, distinction of functional relevance and molecular mechanisms of the involved autophagic components, remains elusive.

\subsection{Distinction of the nucleophagic modes}

The discovery of Atg39 as the selective autophagic cargo receptor for pnER (Mochida et al., 2015) and the subsequent formulation of macronucleophagy instigated novel interpretations of results, previously obtained under the assumption of PMN as the sole mechanism for degradation of nuclear cargo.

Essential requirement for the core autophagic machinery with a subset of proteins conveying selectivity was determined for PMN (Krick et al., 2008). The emergence of macronucleophagy (Mochida et al., 2015), revealed an alternate, selective pathway for degradation of nuclear material, making a reevaluation of previous results necessary. Results obtained for PMN by determination of cargo turnover were implied not to be exclusive for PMN but instead representative of both nucleophagic modes (Mostofa et al., 2018). As a potentially exclusive cargo for PMN, Nvj1 was selected for its putative restriction to the NVJ. While overexpression was shown to result in leakage of the protein into the pnER (Pan 
et al., 2000), expression under its native promoter showed exclusive localisation within the NVJ and absence in macronucleophagic cargo (Figure 4.3).

While data collected in this work supports native levels of Nvj1 as a suitable cargo for measuring PMN turnover, it contradicts the recent assumption of Atg39 as a cargo exclusive for macronucleophagy (Mostofa et al., 2018). Detected in the pnER in general but also clearly within PMN vesicles, Atg39 is present in late stages of PMN, strongly suggesting subsequent degradation by the process (Figure 4.17).

A novel cargo identified for macronucleophagy is nucleoplasm, represented by the $\mathrm{NLS}_{\mathrm{Nab2} 2-\mathrm{mCherry}}$ (Figure 4.1). Previously used for visualisation of cargo for PMN (Krick et al., 2008), the NLS $\mathrm{Nab2}_{2}$ construct could be used in this work for microscopic identification of nucleophagy in general. With approx. $4.9 \mathrm{kDa}$, the atomic mass of the $\mathrm{NLS}_{\mathrm{Nab} 2}$ is quite low when compared to mCherry (approx. 26.7 $\mathrm{kDa}$ ). Discrimination of $\mathrm{NLS}_{\mathrm{Nab} 2}$ and $\mathrm{mCherry}$ would therefore prove difficult when quantifying nucleophagic turnover analogous to the data shown for Nvj1-yEGFP. When using the $\mathrm{NLS}_{\mathrm{Nab2}}$ for quantification of nucleophagic turnover, extension of the protein that does not interfere with localisation might prove to be crucial for conclusive differentiation of fusion protein and protease resistant tag.

\subsubsection{Characterisation of nucleophagic cargo}

In spite of their designated nomenclature, the microautophagic PMN process results in degradation of significantly larger cargo, when compared to the cargo diameter degraded by macronucleophagy (Figure 4.2; Figure 5.2; Figure 5.3). This size difference supports the notion, that macronucleophagy and PMN target cargo of differing composition. While both have been shown to degrade pnER, 
nucleolar protein, nucleoplasm and nucleophagic cargo receptor Atg39, the PMN process exclusively involves degradation of vacuolar membrane and NVJ.

Visualisation of both vacuolar membrane and PMN vesicles was facilitated by expression of $3 \times$ mTagBFP-Pho8 (Figure 4.1). Pho8 was shown to be included in microautophagic degradation (contrasting Vph1) and yet is apparently excluded from late stages of macroautophagic autophagosome vacuole contact sites (Figure 4.8), confirming it as a potentially suitable substrate for determination of microautophagic turnover in general (Hatakeyama \& De Virgilio, 2019; Rahman et al., 2018).

Degradation of vacuolar membrane was previously proposed to enable regulation and maintenance of vacuolar size and composition (Müller et al., 2000). In this model, removal of vacuolar membrane by microautophagy compensates for the continuous membrane influx generated by trafficking pathways such as macroautophagy, that conclude by delivering vesicular membrane to the vacuole.

Analogous to this system, the degradation and thereby regulation of the NVJ and its constituents would describe the primary purpose of PMN. Accumulation and expansion of the NVJ requires a regulatory mechanism. Particularly with regard to resident proteins of the NVJ involved in lipid metabolism and its distinct membrane composition as a putative Lo domain and thereby a potential enrichment in sterols and sphingolipids a form of regulation. Correlation of cellular Nvj1 quantities and size of the NVJ (Pan et al., 2000) alongside heightened PMN rates upon overexpression of Nvj1 (Millen et al., 2009) support a model in which PMN specifically targets the NVJ. This model renders nuclear PMN substrates, in analogy to cytosol in the microautophagic regulation of vacuole size, collateral, and their inclusion merely circumstantial to the topological restriction of the PMN process. 
The apparent size difference between the modes of nucleophagy was previously shown to be at least partially dictated by presence of Tsc13 in the NVJ. Speculated to be an effect of VLCFA enrichment, deletion of Tsc13 and inhibition of de novo fatty acid synthesis and elongation lead to a decrease in PMN cargo diameter, potentially due to the curvature inducing nature of VLCFA of their extended hydrophobic tails (Kvam et al., 2005; Schneiter \& Kohlwein, 1997). Presence of sterol in the NVJ additionally hints at a distinct, possibly more rigid membrane composition for PMN vesicles as opposed to macronucleophagic autophagosomes.

\subsection{Detailed investigations of nucleophagy produce novel insights into selective macroautophagy}

Three dimensional investigations of the autophagic machinery presented amendments to the well established model autophagosome formation. While spherical envelopment of cargo is in line with 2D data and modellation, multiple PAS-like sources of origin are reminiscent of the mammalian autophagic system (Yamada et al., 2005; Young et al., 2006). This was previously thought to be a feature of distinction from the yeast model.

Seemingly required to ensure stable association with the vacuolar membrane, helical wrapping of nuclear cargo by the IM is a novel observation regarding autophagosome formation (Figure 4.5). This might potentially be realised by multiple finger like IMs growing towards each other from various PAS, while remaining associated with portions of the designated cargo (Figure 4.6). The systematic formation of helical structures supports a sophisticated system for coordination of extension, which also presumably requires multiple fusion processes for formation of the spherical autophagosome. 


\subsubsection{Macronucleophagy enables fine mapping of native autophagosome formation}

While significantly smaller than PMN cargo, macroautophagic autophagosome sizes enabled recording of the formation process under native conditions (Figure 4.4). Comparable visualisation of the different stages of autophagosome formation was previously shown under various artificial conditions, by overexpressing Cvt-pathway cargo Ape1 and thereby generating giant Ape1 complexes (Suzuki et al., 2013), or addition of heightened concentrations of rapamycin (Graef et al., 2013). A more recent study presented a method for visualisation of fully formed autophagosomes after rapamycin induction, by structured illumination microscopy (SIM) and subsequent subtraction of background by image processing (Lin et al., 2018).

Systematic formulation of strategies for visualisation of the macroautophagic process, demonstrates the need for a system, that allows for reliable fine mapping of the autophagic machinery within a live cell setup. Discrepancies in the data obtained, using artificial conditions, supports the necessity for a system which is functional under native conditions. Macronucleophagy, as an exemplary process for autophagosome formation in selective macroautophagy, presents a viable alternative to the various mutational or overexpression strategies. Limitations such as the inability to develop into fully formed autophagosomes, requirement for application of specific mutant strains, or specialised microscopy setups and image processing techniques, are not required when observing the nucleophagic process.

As a subtype specific component of the autophagic machinery, Atg11 was not investigated in any of the previously published fine mapping approaches. While localisation of Atg11 at contact sites of cargo and vacuolar membrane is not 
surprising (Figure 4.11), it supports the hypothesis of interaction with Vac8 in the vacuolar membrane (Figure 5.2). Atg11 in turn recruits cargo to the vacuolar membrane in a transient manner, a process that is later reinforced by cargo interaction with the autophagic machinery via Atg8 (Oku et al., 2006).

Presence of two membrane layers upon degradation of pnER by macronucleophagy and three layers of membrane for PMN hints at generally large cargo sizes for overall nucleophagy. Previously observed autophagosome sizes ranging between 150 - $500 \mathrm{~nm}$ (Baba et al., 1997), are in line with the median macronucleophagic autophagosome size of $380 \mathrm{~nm}$ determined in this work (Figure 4.2B). While the value is not exceptionally high, cargo sizes for macronucleophagy enable visualisation of autophagosome biogenesis under native conditions in a widefield fluorescence microscopy setup. While images were additionally processed by deconvolution, raw images obtained from the widefield DeltaVision ${ }^{\circledR}$ setup already depict morphologically distinct steps of autophagosome formation.

For autophagy types with generally small cargo, such as the Cvt-pathway, with vesicular diameter of approx. $150 \mathrm{~nm}$ (Baba et al., 1997) resolution was possible by applying STED microscopy (Figure 4.15). Labelling of fluorescent proteins with fluorophore coupled sdAbs in permeabilised cells and subsequent recording in a STED microscopy setup, presents a suitable alternative to electron microscopy, while maintaining the advantages of multi-channel fluorescence microscopy for visualisation of the autophagic machinery in macroautophagic context. 


\subsubsection{A reduction of Pho8 coincides with late stages of autophagosome formation}

Investigation of the macronucleophagic process in $3 \mathrm{D}$ shows that the isolation membrane is localised at a site within the vacuolar membrane, that contains reduced levels of Pho8. A distinct membrane composition correlated with autophagosome biogenesis implies it as a prerequisite either for formation of the autophagosome or its ultimate fusion with the vacuolar membrane (Figure 5.1). Restriction of these observations to late stages of the macroautophagic process support a requirement for the autophagosomal fusion process. Of course, the membrane portion could also represent the PAS, which might serve a dual function for both recruitment and facilitation of the fusion event.

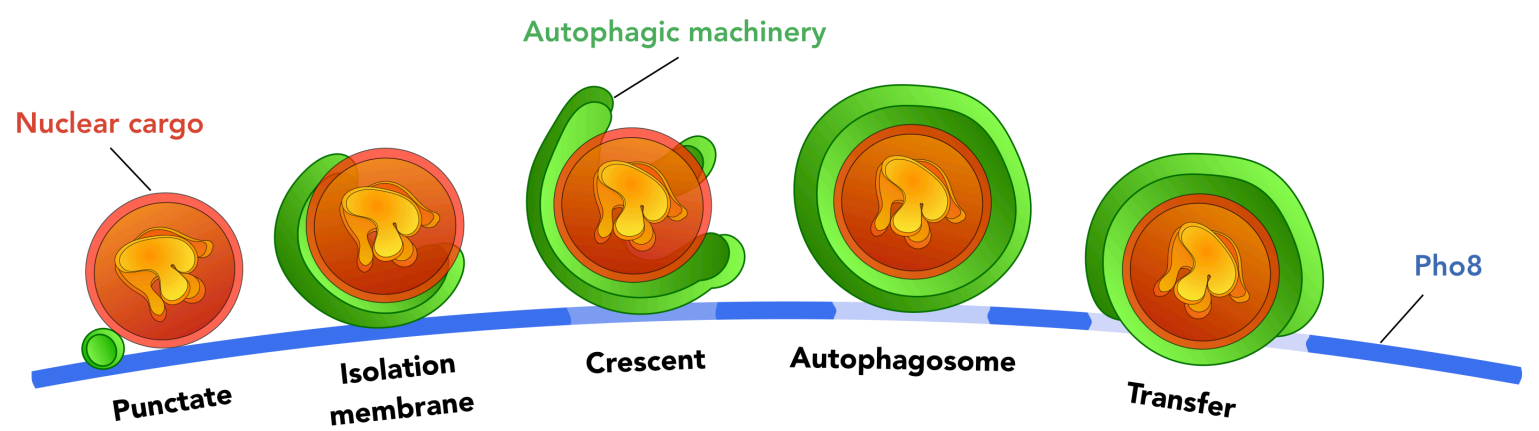

Figure 5.1: Autophagosome biogenesis based on macronucleophagic data

Macronucleophagic investigations revealed a higher complexity of the autophagosomal formation stages obtained from 3D reconstruction of fluorescence microscopy data. A change in vacuole membrane composition at the autophagosome vacuole contact site was shown to coincide with later stages of the biogenesis process (crescent curvature and subsequent stages). All observed transfer stages coincided with a reduction of Pho8 in the vacuolar membrane implying compositional change to be a prerequisite for transfer of autophagosomes into the vacuolar lumen.

The change in membrane composition in a selective autophagic process indicates a general autophagic feature, rather than a nucleophagy specific mechanism. 


\subsection{PMN employs a microautophagic membrane}

\subsubsection{The autophagic machinery at the microautophagic membrane}

Presence of an autophagic structure, containing Atg8 between the vacuolar sequestering arms, in shape of a distinct membrane-like structure is highly reminiscent of the MIPA identified for micropexophagy in P. pastoris (Mukaiyama et al., 2004). In contrast to observations made for the MIPA, a highly curved structure of 1,500 $\mathrm{nm}$ to 2,000 $\mathrm{nm}$ in size (Yamashita et al., 2006), measurements for PMN show a much shorter structure of approx. $200 \mathrm{~nm}$ with a slightly bent outline (Figure 4.16). The large discrepancy in size between PMN and micropexophagy in P. pastoris is likely due to the protocol for induction of pexophagy. Exposure to methanol containing medium, forcing peroxisome accumulation followed by substitution for glucose results in high quantities of peroxisome aggregates. Large micropexophagic cargo diameters are observed as a consequence, permitting convenient resolution in fluorescence microscopy, but potentially influencing size of the MIPA, which is presumably smaller for native cargo quantities.

Highly curved membranes, such as the vacuolar sequestering arms present at either side of the microautophagic membrane, invite speculation for recruitment of curvature sensing members of the autophagic machinery. Curvature sensing was shown for the C-terminal EAT domain of Atg1, for ATG14L in humans and Atg3, that has also shown preference for PE enriched membranes (Rao et al., 2016; Fan et al., 2011; Hanada et al., 2009; Nath et al., 2014). In context of PMN, these proteins could initiate the process, after motor protein mediated bulging. As the E2-like enzyme for Atg8 lipidation, localisation of Atg3 at the vacuolar tips could be a prerequisite for Atg8 recruitment and thereby formation of the microautophagic membrane apparatus. 
In micropexophagy, fusion of the MIPA with the vacuolar arms was implied to function dependent on the SNX PpAtg24 (Ano et al., 2005). Partial involvement of its S. cerevisiae counterpart in PMN was previously suggested (Krick et al., 2008). Discovery of the microautophagic membrane in PMN implies a similar localisation of Atg24 at the tips of the vacuolar arms in PMN context. Its partial involvement in PMN also implies an at least partially redundant role of Atg24, which supports involvement of alternative components for the fusion process. Complex formation of Atg24 with the SNX Atg20 is essential for the Cvt-pathway and requires binding of Atg20 to Atg11. Whereas Atg11 is present between the vacuolar arms (Figure 4.19; Figure 5.3), the fact that Atg20 is redundant for nucleophagy (Krick et al., 2008) suggests interaction with an alternative component. A shared feature of the SNX proteins Atg24 and Atg20 are their respective PX domains (Popelka et al., 2017). PX domains are also present in nuclear vacuole tether Mdm1 (Henne et al., 2015), which was observed to accumulate at the edges of the NVJ and has a designated and highly conserved role as an SNX, implying a potentially essential function. Characteriation of functionality within the PMN process, possibly by knockout alongside Atg24, could further unravel the mechanism for generation and fusion of the newly discovered microautophagic membrane. Another alternative is Ypt35, the SNX required for recruitment of NVJ resident protein Vps13, which also contains the shared PX domain (Vollert \& Uetz, 2004).

Tethering functionality and involvement in hemifusion were shown for Atg8 and its mammalian orthologs LC3 and GABARAP in vitro, but have been discussed controversially in in vivo context (Nakatogawa et al., 2007; Weidberg et al., 2010; Nair et al., 2011; Landajuela et al., 2016). A role in tethering the vacuolar arms surrounding the PMN vesicle appears possible, yet the curved structure of the membrane also implies relation to the IM and thereby potential recruitment of tethers involved in macroautophagy. While the homotypic fusion machinery was 
shown not to be involved in PMN, alternative tethers have yet to be investigated (Krick et al., 2008; 2009a; Millen et al., 2009).

\subsubsection{Composition of the microautophagic membrane}

Mechanistically distinct from PMN, micropexophagy in $P$. pastoris requires septation and formation of the VSM. The MIPA was shown to require and contain Atg26, which is in contrast not involved in PMN (Krick et al., 2008), a PI4P and sterol binding protein that also implies a distinct lipid composition (Yamashita et al., 2007). Discovery of a membrane at the site of vacuolar membrane encounter in PMN implicates the MIPA not as an exclusively characteristic feature for micropexophagy in $P$. pastoris, but indicates the requirement for a microautophagic membrane to be conserved within the microautophagic mechanism in general. Presence of PI3P could not be shown at the microautophagic membrane in PMN but only at non-NVJ portions of PMN vesicles (Figure 4.20). Its overall absence from the NVJ implies, that the inability of the GFP-FYVEEEA1 construct to reach the junction is responsible for the absence of binding, rather than an actual lack of PI3P within the microautophagic process.

Protein precursors integral to the vacuolar membrane such as Pho8 are known to interact with and require processing by vacuolar hydrolases. The mechanism by which the inner vacuolar membrane is shielded from degradation by resident lipases, represented thus far by Atg15, has yet to be uncovered (Takeshige et al., 1992). Presence of an Atg8 containing membrane, provides potential access for vacuolar hydrolases. In this case, hydrolases might bypass the inner vacuolar membrane by degradation of the microautophagic membrane (Figure 5.3), which shares similarities with the autophagosomal membrane, a known substrate of vacuolar degradation. Degradation of microautophagic cargo from the inside also 
presents a resolution to the ongoing conundrum that is the selective degradation of microautophagic vesicles coinciding with preservation of their vacuolar source.

\subsection{Atg39 assumes a central role in nucleophagy}

Investigations regarding Atg39 verified the presumed role (Mochida et al., 2015) for Atg39 as the macronucleophagic cargo receptor. In addition, Atg39 shows involvement in PMN, reintroducing it as the overall nucleophagic cargo receptor.

\subsubsection{Further characterisation of Atg39 in macronucleophagic context}

Continuous localisation of Atg39 on the outside of nucleoplasmic cargo confirms the interpretation of Mochida et al. (2015), that inferred the double-ringed structure observed by electron microscopy in nucleophagic cargo to be ONM and INM (Figure 4.9). This localisation also suggests a budding mechanism similar to that, observed for PMN in which micronuclei bud off from the main nucleus.

Significant accumulation of Atg39 around macronucleophagic cargo implies functional relevance (Figure 4.10). Accumulation of the mammalian cER-phagy receptor and Atg40 homologue FAM134B was recently shown to be required for functional cER-phagy (Bhaskara et al., 2019). FAM134B accumulation actively induces bulging in the ER membrane via its reticulon homology domain (RHD). While Atg39 does not contain an RHD, tandem recruitment of curvature inducing factors by Atg39 might present a mechanism for initiation of macronucleophagy.

Similar to the microautophagic membrane observed in PMN, the role for Atg39 appears to be highly reminiscent of pexophagy receptor PpAtg30 in P. pastoris. PpAtg30 is required for both macro- and micropexophagy (Farré et al., 2008; 
Farré et al., 2013) which resembles the situation observed for nucleophagy in this work.

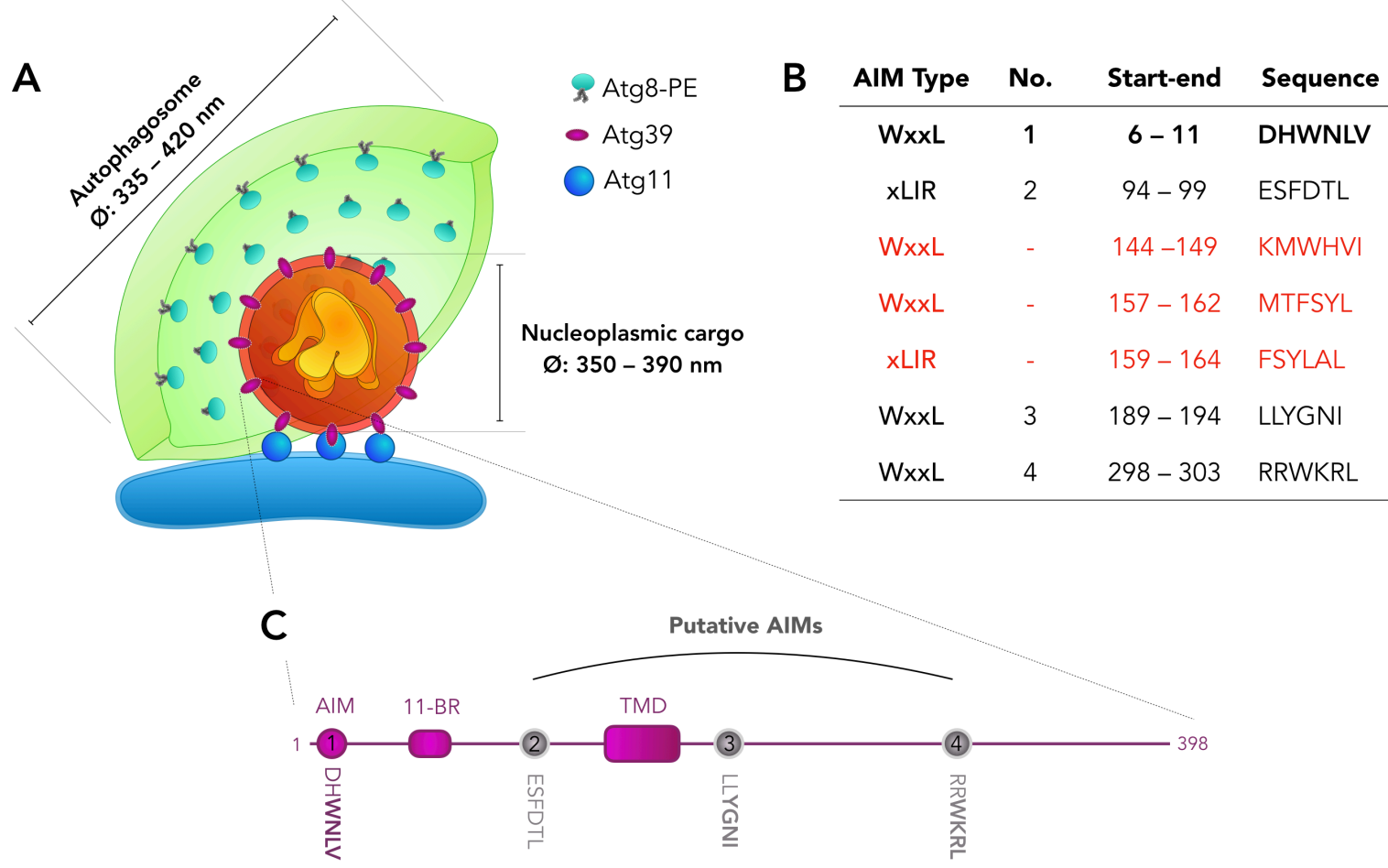

Figure 5.2: Integration of novel data into the model of macronucleophagy

(A) Data collected in this work depicts localisation of Atg39 on the outside of the nucleoplasm containing autophagic cargo. A presumed interaction of the 11-BR of Atg39, with cargo adaptor Atg11 was observed predominantly at sites of overlap between vacuolar membrane and nucleophagic cargo. As expected, Atg8 was generally located around the cargo signal progressively forming the autophagosomal strucure. Nucleoplasmic cargo sizes were experimentally determined alongside autophagosomal diameters. (B) Analysis of the Atg39 sequence revealed additional AIMs present at the cytosolic and ER-lumen part of the protein. AIMs located within the transmembrane domain are depicted in red presumably excluded from functional capacity. AIM no. 1, the most aminoterminally located AIM was confirmed to be required for macronucleophagic functionality (Mochida et al., 2015). (C) The Atg39 protein sequence shows that four of the putative AIMs predicted outside of the TMD, two are located on the cytosolic side and two are located in the larger ER-lumen side.

\subsubsection{Atg39 is functionally involved in PMN}

Microscopic investigation of Atg39 in PMN showed localisation of Atg39 at the MIPA-like structure with additional signals in the pnER. Accumulation at the microautophagic membrane implies interaction with and potential recruitment of 
Atg8 within the microautophagic process. Deletion of Atg39 leads to a significant reduction of Nvj1 degradation and thereby supports a model of PMN with functional involvement of Atg39 (Figure 4.18).

As the potential cargo receptor for both macro- and micronucleophagy, Atg39 is reminiscent of PpAtg30, which is required for both macro- and micropexophagy in P. pastoris (Farré et al., 2008). Overall similarities in the two processes and presence at the microautophagic membrane support a role for Atg39 as the designated cargo receptor for PMN.

In addition to its confirmed AIM required for functional macroautophagy located between the 6-11 aa (Mochida et al., 2015), Atg39 contains six additional putative AlM motifs (Figure 5.2B \& C). While three are located within the TMD, one is present on the cytosolic side and another two are predicted to be at the side of the ER-lumen. While putative AIM sequences are highly abundant even when applying the more sophisticated XLIR prediction, they do not necessarily represent functional AIMs, yet separate AIM usage for macronucleophagy and PMN could present an intriguing regulatory mechanism for distinct initiation of the two spatially and temporally distinct processes. Accessibility of the lumenar AlMs could be restricted to the fission process of PMN and would precondition nuclear presence of Atg8, which was previously shown for Atg8 and LC3 (Drake et al., 2010; Huang et al., 2015) and also observed in this work.

Recruitment to the autophagic machinery of Atg39 is mediated in addition to its functional AIM, by interaction with Atg11 via its 11-BR. Involvement of Atg11 in both nucleophagic modes not only reaffirms the notion of their selective modality but also adds significance to the function of the cargo adaptor, suggesting further investigation. Observed for the 11-BR containing cargo receptors Atg19, Atg34 and Atg36, interaction with Atg11 is conditioned by Hrr25 (Tanaka et al., 2014; 
Pfaffenwimmer et al., 2014). Phosphorylation of serine residues within the binding site is mediated by Hrr25. The 11-BR of Atg39 contains four serines as potential targets for Hrr25 phosphorylation. Involvement of Atg39 and Atg11 in PMN supports a potential role for Hrr25 in the nucleophagic process with particular respect to its analogies regarding pexophagy and further investigation should be considered.
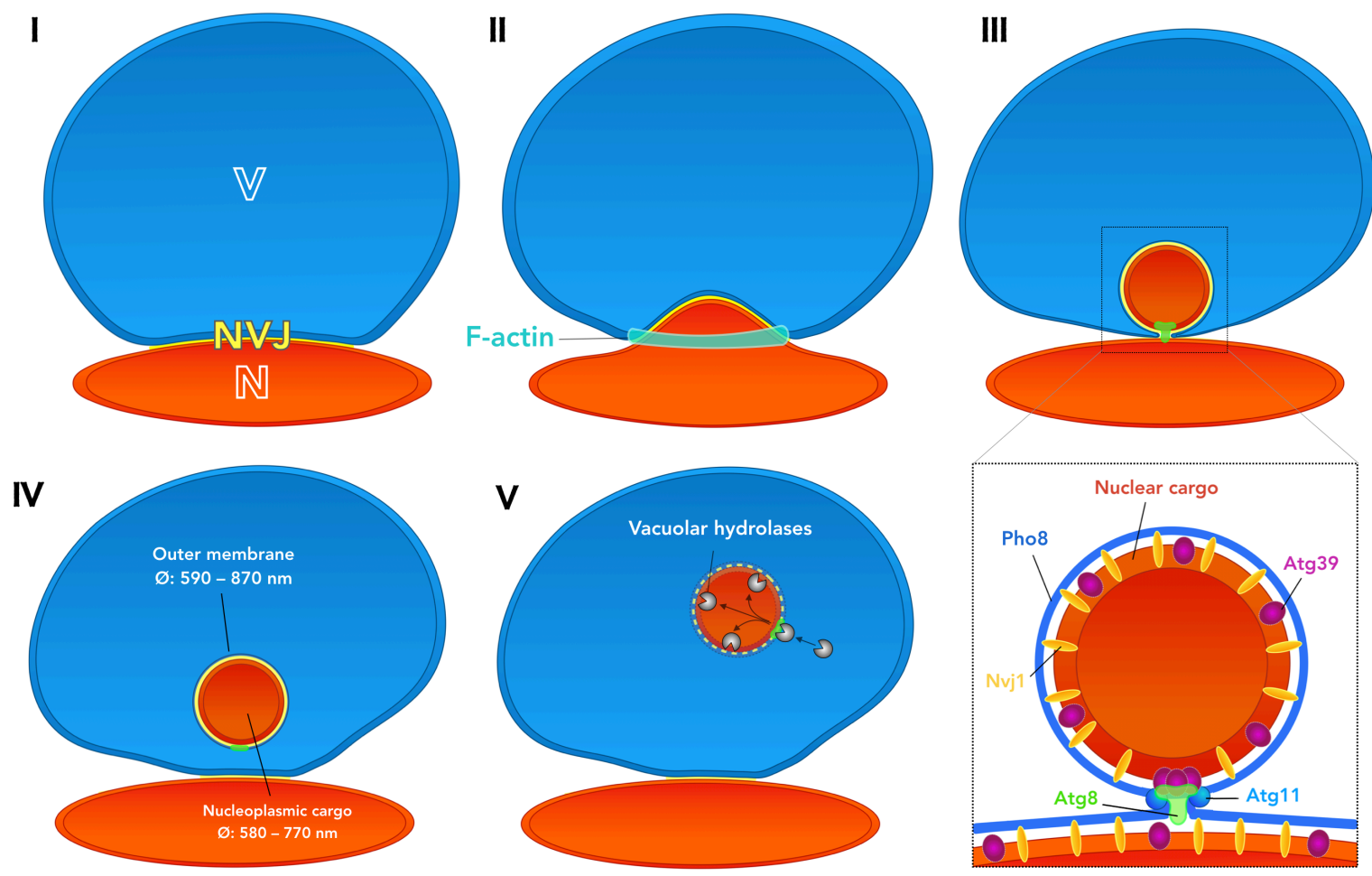

\section{Figure 5.3: Proposed model for PMN implementing novel data}

In the updated model for PMN the five steps previously proposed for the process are shown incorporating data observed in the process of this work. The NVJ (I) is degraded in a stepwise process. Initial bulging (II) was shown to involve a ring like F-actin structure. Formation of the PMN vesicle (III) involves accumulation of Atg39 at the tips of the vacuolar arms and presumably recruitment of Atg8 and Atg11. Formation of the microautophagic membrane is succeeded by release of the vesicle into the vacuolar lumen (IV). Shown to be significantly larger in diameter, PMN vesicles were shown to contain an Atg8 positive microautophagic membrane structure even upon release into the vacuole. The microautophagic membrane was speculated to allow access for vacuolar hydrolyses into the cargo vesicle (V), that is otherwise shielded by vacuolar membrane. 


\subsubsection{Atg39 links PMN to the regulation of lipid metabolism}

A shared feature of Atg39 and Nvj1 is, that the upstream regions of both ORFs contain two STRE repeats, resulting in upgregulated expression during stationary phase and nutrient deprivation (Moskvina et al., 1998; Gasch et al., 2000; Roberts et al., 2003). Expressional upregulation by the same mechanism is supportive of involvement in the same process, in this case PMN. Since length of the NVJ is directly proportional to cellular levels of Nvj1 (Pan et al., 2000; Roberts et al., 2003) and reduction of the NVJ and regulation of its constituents is presumably mediated by PMN which is in turn dependent on Atg39, concurrent expression of Nvj1 and Atg39 presents a potential regulatory mechanism for prevention of pathological enlargement of the NVJ (Figure 5.3).

Although provision of Atg39 might simply be a prerequisite for potential degradation of the NVJ, actual initiation of PMN could be mediated by other factors, such as sensing an extension limit of the NVJ, or possibly downstream effects of NVJ resident proteins.

As a major storage site for sterols, LDs are also involved in sterol transport and degradation in the vacuole and were shown to actively accumulate at the NVJ. Mediated by Mdm1 and colocalising with the nuclear vacuolar tether LDs accumulate and are partially synthesised at the NVJ prior to degradation by microlipophagy (Hariri et al., 2018; Hariri et al., 2019). Coinciding with its identification as an autophagic cargo receptor, Atg39 was identified as the ERstress-induced microlipophagy gene 1, Esm1 (Vevea et al., 2015). Noticeably involved in microlipophagy and thought to be a part of the ESCRT machinery, Atg39 was shown to be required for adaption to lipid imbalance and is activated by lipid and ER stress, linking its functionality for degradation of the pnER and NVJ. 
Redistribution of Atg39 upon LD accumulation and localisation at LD vacuole contact sites supports a role for Atg39 in LD regulation (Figure 4.23). Relocalisation of Atg39 extending from the pnER might be a consequence of pnER redistribution. While the extensions did not contain nucleoplasmic material, organisation of LD recruitment could be mediated by the pnER and not necessarily restricted to Atg39 since its localisation in surrounding nucleoplasm remains unchanged. Changes in nuclear morphology were previously shown to precede nucleophagy (Rahman et al., 2018). Both production of diacylglycerols (DAGs), localisation of Nvj1 and nuclear morphology were shown to be mediated by the Nem1/Spo7-Pah1 axis. Synthesis of TAGs at the nucleus was shown to be coordinated by Lro1 within the INM. This synthesis occurs at a nuclear subdomain which accumulates Lro1 and requires recruitment of the nucleolus (Barbosa et al., 2019). Nucleophagic degradation of Nop1 might therefore be a consequence of regulation of the TAG synthesis domain by nucleophagy. Recruitment of LDs for delivery of TAGs might be localised at the domain and their recruitment could be facilitated by factors of the pnER such as Mdm1 and Atg39.

While nucleophagy and lipid metabolism as well as lipophagy appear clearly interlinked, the nature of Atg39 within the process requires further research to determine direct or indirect involvement of the cargo receptor. Effects of Atg39 in LD synthesis and degradation might be mediated not by direct interaction or delivery, but by proxy: Via regulation of the major lipid metabolism hub which is the NVJ.

\subsection{Motor proteins are involved in the initial steps of PMN}

Shown to play a role during autophagosome formation in mammalian cells (Aplin et al.,1992), actin was shown to be specifically involved in selective autophagy (Reggiori et al., 2005). In particular, autophagic degradation of ER was 
determined to be dependent on actin (Hamasaki et al., 2005), with Atg9 no longer reaching the PAS upon its disruption (Reggiori et al., 2005).

Application of F-actin depolymerising drugs, such as cytochalasin D and latrunculin B, were shown to inhibit autophagosome formation (Aplin et al., 1992). Several actin based motor proteins, including myosin I, II and VI, were associated with autophagy (Kruppa et al., 2016). They were also shown to interact with sterol-enriched lipid rafts, regulating cellular sterol homeostasis (Brandstaetter et al., 2012), representing an essential prerequisite for autophagosome lysosome fusion (Fraldi et al., 2010).

Overall investigation of motor protein involvement was so far focused on macroautophagic processes. Association of a contractile ring with the initiation of PMN presents a novel feature for microautophagy and could also be representative of the actin dependent degradation of $E R$, which was previously observed (Figure 4.21; Figure 5.3; Hamasaki et al., 2005).

Further investigations of motor protein involvement in PMN should be considered with regard to the effect of F-actin depolymerising drugs, myosin localisation as well as mutation of components required for contractile ring formation. 


\section{Outlook}

Identification of a novel membrane structure suggests requirement for the autophagic machinery between vacuolar arms as a general feature of microautophagy. A detailed investigation of the structure, in compositional reference with its macroautophagic counterpart should be carried out to further unravel the underlying mechanisms of the lesser investigated system, that is microautophagy.

A requirement for motor proteins in PMN, especially in early bulging of the nucleus demands further investigation. Potential involvement of myosins, as the corresponding element to actin in contractile rings and previous hints at involvement of the protein in PMN, could present a starting point for advancing the understanding of motor protein involvement in autophagy.

As a cargo of PMN and a hub for various proteins involved in lipid synthesis and distribution, previous and present data of the NVJ suggest it as a sterol enriched membrane, possibly qualifying as $\mathrm{L}_{\mathrm{O}}$ domain. Potentially requirement of PMN and by extension Atg39 for regulation of LD synthesis and recycling, as well as sterol accumulation and distribution, heavily imply a link for nucleophagy and lipid homeostasis in the cell. Identification of the unknown neutral lipid population upon deletion of Atg39 should further detail its involvement in the metabolic process.

Novel insights into native autophagosome formation via macronucleophagy enables observation of detailed localisations of the autophagic machinery within the biogenesis process and presents a viable alternative to approaches implementing cargo overabundance. In addition, spacial evaluation by three dimensional investigation of autophagosome was shown to precondition a 
comprehensive understanding of the mechanistic intricacies of the autophagic system.

Autophagic modes targeting cargo with a diameter smaller than $200 \mathrm{~nm}$ were shown to be efficiently visualised beyond the resolution limits of conventional fluorescence microscopy by sdAb-mediated STED microscopy, opening up various opportunities for even more detailed research into the autophagic system and differentiation of its subcomponents. 


\section{Bibliography}

Aaij, C., \& Borst, P. (1972). The gel electrophoresis of DNA. Biochimica et Biophysica Acta (BBA)Nucleic Acids and Protein Synthesis, 269(2), 192-200.

Agarraberes, F. A., \& Dice, J. F. (2001). A molecular chaperone complex at the lysosomal membrane is required for protein translocation. J Cell Sci, 114(13), 2491-2499.

Ahlberg, J., Marzella, L., \& Glaumann, H. (1982). Uptake and degradation of proteins by isolated rat liver lysosomes. Suggestion of a microautophagic pathway of proteolysis. Lab Invest, 47(6), 523-532.

Akinduro, O., Sully, K., Patel, A., Robinson, D. J., Chikh, A., McPhail, G., ... \& O'Shaughnessy, R. F. (2016). Constitutive autophagy and nucleophagy during epidermal differentiation. J Invest Dermatol, 136(7), 1460-1470.

Ammerer, G., Hunter, C. P., Rothman, J. H., Saari, G. C., Valls, L. A., \& Stevens, T. H. (1986). PEP4 gene of Saccharomyces cerevisiae encodes proteinase $A$, a vacuolar enzyme required for processing of vacuolar precursors. Mol Cell Biol, 6(7), 2490-2499.

Ano, Y., Hattori, T., Oku, M., Mukaiyama, H., Baba, M., Ohsumi, Y., ... \& Sakai, Y. (2005). A sorting nexin PpAtg24 regulates vacuolar membrane dynamics during pexophagy via binding to phosphatidylinositol-3-phosphate. Mol Biol Cell, 16(2), 446-457.

Aplin, A., Jasionowski, T., Tuttle, D. L., Lenk, S. E., \& Dunn Jr, W. A. (1992). Cytoskeletal elements are required for the formation and maturation of autophagic vacuoles. J Cell Physiol, 152(3), 458-466.

Araki, Y., Ku, W. C., Akioka, M., May, A. I., Hayashi, Y., Arisaka, F., .. \& Ohsumi, Y. (2013). Atg38 is required for autophagy-specific phosphatidylinositol 3-kinase complex integrity. J Cell Biol, 203(2), 299-313.

Ashkenazi, A., Bento, C. F., Ricketts, T., Vicinanza, M., Siddiqi, F., Pavel, M., ... \& Rubinsztein, D. C. (2017). Polyglutamine tracts regulate beclin 1-dependent autophagy. Nature, 545(7652), 108.

Baba, M., Osumi, M., Scott, S. V., Klionsky, D. J., \& Ohsumi, Y. (1997). Two distinct pathways for targeting proteins from the cytoplasm to the vacuole/lysosome. J Cell Biol, 139(7), 1687-1695.

Bandyopadhyay, U., Sridhar, S., Kaushik, S., Kiffin, R., \& Cuervo, A. M. (2010). Identification of regulators of chaperone-mediated autophagy. Mol Cell, 39(4), 535-547.

Bao, Z., HamediRad, M., Xue, P., Xiao, H., Tasan, I., Chao, R., ... \& Zhao, H. (2018). Genome-scale engineering of Saccharomyces cerevisiae with single-nucleotide precision. Nat Biotechnol, 36(6), 505.

Barbosa, A. D., Lim, K., Mari, M., Edgar, J. R., Gal, L., Sterk, P., .. \& Reggiori, F. (2019). Compartmentalized Synthesis of Triacylglycerol at the Inner Nuclear Membrane Regulates Nuclear Organization. Dev Cell, in press. 
Barbosa, A. D., Sembongi, H., Su, W. M., Abreu, S., Reggiori, F., Carman, G. M., \& Siniossoglou, S. (2015). Lipid partitioning at the nuclear envelope controls membrane biogenesis. Mol Biol Cell, 26(20), 3641-3657.

Baron, O., Boudi, A., Dias, C., Schilling, M., Nölle, A., Vizcay-Barrena, G., .. \& Bates, G. P. (2017). Stall in canonical autophagy-lysosome pathways prompts nucleophagy-based nuclear breakdown in neurodegeneration. Curr Biol, 27(23), 3626-3642.

Bas, L., Papinski, D., Licheva, M., Torggler, R., Rohringer, S., Schuschnig, M., \& Kraft, C. (2018). Reconstitution reveals Ykt6 as the autophagosomal SNARE in autophagosome-vacuole fusion. $J$ Cell Biol, 217(10), 3656-3669.

Bean, B. D., Dziurdzik, S. K., Kolehmainen, K. L., Fowler, C. M., Kwong, W. K., Grad, L. I., ... \& Conibear, E. (2018). Competitive organelle-specific adaptors recruit Vps13 to membrane contact sites. J Cell Biol, 217(10), 3593-3607.

Begley, M. J., Taylor, G. S., Kim, S. A., Veine, D. M., Dixon, J. E., \& Stuckey, J. A. (2003). Crystal structure of a phosphoinositide phosphatase, MTMR2: insights into myotubular myopathy and Charcot-Marie-Tooth syndrome. Mol Cell, 12(6), 1391-1402.

Bhaskara, R. M., Grumati, P., Garcia-Pardo, J., Kalayil, S., Covarrubias-Pinto, A., Chen, W., ... \& Hummer, G. (2019). Curvature induction and membrane remodeling by FAM134B reticulon homology domain assist selective ER-phagy. Nat Commun, 10(1), 2370.

Bi, E., Maddox, P., Lew, D. J., Salmon, E. D., McMillan, J. N., Yeh, E., \& Pringle, J. R. (1998). Involvement of an actomyosin contractile ring in Saccharomyces cerevisiae cytokinesis. J Cell Biol, 142(5), 1301-1312.

Bimboim, H. C., \& Doly, J. (1979). A rapid alkaline extraction procedure for screening recombinant plasmid DNA. Nucleic Acids Res, 7(6), 1513-1523.

Binda, M., Péli-Gulli, M. P., Bonfils, G., Panchaud, N., Urban, J., Sturgill, T. W., ... \& de Virgilio, C. (2009). The Vam6 GEF controls TORC1 by activating the EGO complex. Mol Cell, 35(5), 563-573.

Birgisdottir, Å. B., Lamark, T., \& Johansen, T. (2013). The LIR motif-crucial for selective autophagy. J Cell Sci, 126(15), 3237-3247.

Bonfils, G., Jaquenoud, M., Bontron, S., Ostrowicz, C., Ungermann, C., \& de Virgilio, C. (2012). Leucyl-tRNA synthetase controls TORC1 via the EGO complex. Mol Cell, 46(1), 105-110.

Bouchard, L., Faucher, G., Tchernof, A., Deshaies, Y., Marceau, S., Lescelleur, O., ... \& Vohl, M. C. (2009). Association of OSBPL11 gene polymorphisms with cardiovascular disease risk factors in obesity. Obesity, 17(7), 1466-1472.

Boyer, H. W. (1971). DNA restriction and modification mechanisms in bacteria. Annu Rev Microbiol, 25(1), 153-176.

Brandstaetter, H., Kendrick-Jones, J., \& Buss, F. (2012). Myo1c regulates lipid raft recycling to control cell spreading, migration and Salmonella invasion. J Cell Sci, 125(8), 1991-2003. 
Brickner, J. H., \& Fuller, R. S. (1997). SOl1 encodes a novel, conserved protein that promotes TGN-endosomal cycling of Kex $2 p$ and other membrane proteins by modulating the function of two TGN localization signals. J Cell Biol, 139(1), 23-36.

Bryant, D., Liu, Y., Datta, S., Hariri, H., Seda, M., Anderson, G., ... \& Clayton, P. (2018). SNX14 mutations affect endoplasmic reticulum-associated neutral lipid metabolism in autosomal recessive spinocerebellar ataxia 20. Hum Mol Genet, 27(11), 1927-1940.

Budovskaya, Y. V., Stephan, J. S., Deminoff, S. J., \& Herman, P. K. (2005). An evolutionary proteomics approach identifies substrates of the cAMP-dependent protein kinase. Proc Natl Acad Sci U.S.A, 102(39), 13933-13938.

Budovskaya, Y. V., Stephan, J. S., Reggiori, F., Klionsky, D. J., \& Herman, P. K. (2004). The Ras/ cAMP-dependent protein kinase signaling pathway regulates an early step of the autophagy process in Saccharomyces cerevisiae. J Biol Chem, 279(20), 20663-20671.

Burd, C. G., \& Emr, S. D. (1998). Phosphatidylinositol (3)-phosphate signaling mediated by specific binding to RING FYVE domains. Mol Cell, 2(1), 157-162.

Burnett, S. F., Farré, J. C., Nazarko, T. Y., \& Subramani, S. (2015). Peroxisomal Pex3 activates selective autophagy of peroxisomes via interaction with the pexophagy receptor Atg30. J Biol Chem, 290(13), 8623-8631.

Cebollero, E., van der Vaart, A., Zhao, M., Rieter, E., Klionsky, D. J., Helms, J. B., \& Reggiori, F. (2012). Phosphatidylinositol-3-phosphate clearance plays a key role in autophagosome completion. Curr Biol, 22(17), 1545-1553.

Cheng, J., Fujita, A., Yamamoto, H., Tatematsu, T., Kakuta, S., Obara, K., ... \& Fujimoto, T. (2014). Yeast and mammalian autophagosomes exhibit distinct phosphatidylinositol 3-phosphate asymmetries. Nat Commun, 5, 3207.

Cheong, H., Nair, U., Geng, J., \& Klionsky, D. J. (2008). The Atg1 kinase complex is involved in the regulation of protein recruitment to initiate sequestering vesicle formation for nonspecific autophagy in Saccharomyces cerevisiae. Mol Biol Cell, 19(2), 668-681.

Cherry, J. M., Hong, E. L., Amundsen, C., Balakrishnan, R., Binkley, G., Chan, E. T., .. \& Fisk, D. G. (2011). Saccharomyces Genome Database: the genomics resource of budding yeast. Nucleic Acids Res, 40(1), 700-705.

Chew, L. H., Lu, S., Liu, X., Li, F. K., Yu, A. Y., Klionsky, D. J., ... \& Yip, C. K. (2015). Molecular interactions of the Saccharomyces cerevisiae Atg1 complex provide insights into assembly and regulatory mechanisms. Autophagy, 11(6), 891-905.

Chew, L. H., Setiaputra, D., Klionsky, D. J., \& Yip, C. K. (2013). Structural characterization of the Saccharomyces cerevisiae autophagy regulatory complex Atg17-Atg31-Atg29. Autophagy, 9(10), 1467-1474.

Chung, J., Torta, F., Masai, K., Lucast, L., Czapla, H., Tanner, L. B., ... \& de Camilli, P. (2015). PI4P/ phosphatidylserine countertransport at ORP5-and ORP8-mediated ER-plasma membrane contacts. Science, 349(6246), 428-432. 
Cicchini, M., Chakrabarti, R., Kongara, S., Price, S., Nahar, R., Lozy, F., .. \& Karantza, V. (2014). Autophagy regulator BECN1 suppresses mammary tumorigenesis driven by WNT1 activation and following parity. Autophagy, 10(11), 2036-2052.

Ciechomska, I. A., \& Tolkovsky, A. M. (2007). Non-autophagic GFP-LC3 puncta induced by saponin and other detergents. Autophagy, 3(6), 586-590.

Cuervo, A. M., Dice, J. F., \& Knecht, E. (1997). A population of rat liver lysosomes responsible for the selective uptake and degradation of cytosolic proteins. J Biol Chem, 272(9), 5606-5615.

Cuervo, A. M., \& Wong, E. (2014). Chaperone-mediated autophagy: roles in disease and aging. Cell Res, 24(1), 92.

Darsow, T., Rieder, S. E., \& Emr, S. D. (1997). A multispecificity syntaxin homologue, Vam3p, essential for autophagic and biosynthetic protein transport to the vacuole. J Cell Biol, 138(3), 517-529.

Datta, S., Liu, Y., Hariri, H., Bowerman, J., \& Henne, W. M. (2019). Cerebellar ataxia diseaseassociated Snx14 promotes lipid droplet growth at ER-droplet contacts. J Cell Biol, 218 (4), $1335-1351$

Davis, S., \& Ferro-Novick, S. (2015). Ypt1 and COPII vesicles act in autophagosome biogenesis and the early secretory pathway. Biochem Soc Trans, 43(1), 92-96.

Dawaliby, R., \& Mayer, A. (2010). Microautophagy of the nucleus coincides with a vacuolar diffusion barrier at nuclear-vacuolar junctions. Mol Biol Cell, 21(23), 4173-4183.

de Duve, C. (1963). The lysosome. Scientific American, 208(5), 64-73.

de Duve, C., \& Wattiaux, R. (1966). Functions of lysosomes. Annual Rev Physiol, 28(1), 435-492.

de Saint-Jean, M., Delfosse, V., Douguet, D., Chicanne, G., Payrastre, B., Bourguet, W., ... \& Drin, G. (2011). Osh4p exchanges sterols for phosphatidylinositol 4-phosphate between lipid bilayers. $J$ Cell Biol, 195(6), 965-978.

Delorme-Axford, E., Guimaraes, R. S., Reggiori, F., \& Klionsky, D. J. (2015). The yeast Saccharomyces cerevisiae: an overview of methods to study autophagy progression. Methods, 75, 3-12.

Dietrich, L. E., \& Ungermann, C. (2004). On the mechanism of protein palmitoylation. EMBO Rep, 5(11), 1053-1057.

Dilcher, M., Köhler, B., \& von Mollard, G. F. (2001). Genetic Interactions with the Yeast Q-SNARE VTI1 Reveal Novel Functions for the R-SNARE YKT6. J Biol Chem, 276(37), 34537-34544.

Dou, Z., Xu, C., Donahue, G., Shimi, T., Pan, J. A., Zhu, J., ... \& Catanzaro, J. M. (2015). Autophagy mediates degradation of nuclear lamina. Nature, 527(7576), 105.

Drake, K. R., Kang, M., \& Kenworthy, A. K. (2010). Nucleocytoplasmic distribution and dynamics of the autophagosome marker EGFP-LC3. PloS one, 5(3), e9806.

Dreyer, T. (1989). Substrate specificity of proteinase yscA from Saccharomyces cerevisiae. Carlsberg Res Commun, 54(3), 85. 
Dubouloz, F., Deloche, O., Wanke, V., Cameroni, E., \& de Virgilio, C. (2005). The TOR and EGO protein complexes orchestrate microautophagy in yeast. Mol Cell, 19(1), 15-26.

Eisenberg-Bord, M., Mari, M., Weill, U., Rosenfeld-Gur, E., Moldavski, O., Castro, I. G., .. \& Reggiori, F. (2018). Identification of seipin-linked factors that act as determinants of a lipid droplet subpopulation. J Cell Biol, 217(1), 269-282.

Epple, U. D., Suriapranata, I., Eskelinen, E. L., \& Thumm, M. (2001). Aut5/Cvt17p, a putative lipase essential for disintegration of autophagic bodies inside the vacuole. J Bacteriol, 183(20), 5942-5955.

Fader, C. M., Sánchez, D. G., Mestre, M. B., \& Colombo, M. I. (2009). TI-VAMP/VAMP7 and VAMP3/cellubrevin: two V-SNARE proteins involved in specific steps of the autophagy/ multivesicular body pathways. Biochimica et Biophysica Acta (BBA)-Molecular Cell Research, 1793(12), 1901-1916.

Færgeman, N. J., Feddersen, S., Christiansen, J. K., Larsen, M. K., Schneiter, R., Ungermann, C., ... \& Knudsen, J. (2004). Acyl-CoA-binding protein, Acb1p, is required for normal vacuole function and ceramide synthesis in Saccharomyces cerevisiae. Biochem J, 380(3), 907-918.

Fan, W., Nassiri, A., \& Zhong, Q. (2011). Autophagosome targeting and membrane curvature sensing by Barkor/Atg14 (L). Proc Natl Acad Sci U.S.A, 108(19), 7769-7774.

Farré, J. C., Burkenroad, A., Burnett, S. F., \& Subramani, S. (2013). Phosphorylation of mitophagy and pexophagy receptors coordinates their interaction with Atg8 and Atg11. EMBO Rep, 14(5), 441-449.

Farré, J. C., Manjithaya, R., Mathewson, R. D., \& Subramani, S. (2008). PpAtg30 tags peroxisomes for turnover by selective autophagy. Dev Cell, 14(3), 365-376.

Fidler, D. R., Murphy, S. E., Courtis, K., Antonoudiou, P., El-Tohamy, R., lent, J., \& Levine, T. P. (2016). Using HHsearch to tackle proteins of unknown function: A pilot study with PH domains. Traffic, 17(11), 1214-1226.

Fischer von Mollard, G., \& Stevens, T. H. (1999). The Saccharomyces cerevisiae v-SNARE Vti1p is required for multiple membrane transport pathways to the vacuole. Mol Biol Cell, 10(6), 1719-1732.

Föller, M., Hermann, A., Gu, S., Alesutan, I., Qadri, S. M., Borst, O., .. \& Schöls, L. (2012). Chorein-sensitive polymerization of cortical actin and suicidal cell death in chorea-acanthocytosis. FASEB J, 26(4), 1526-1534.

Fraldi, A., Annunziata, F., Lombardi, A., Kaiser, H. J., Medina, D. L., Spampanato, C., ... \& Ballabio, A. (2010). Lysosomal fusion and SNARE function are impaired by cholesterol accumulation in lysosomal storage disorders. EMBO J, 29(21), 3607-3620.

Fratti, R. A., Jun, Y., Merz, A. J., Margolis, N., \& Wickner, W. (2004). Interdependent assembly of specific regulatory lipids and membrane fusion proteins into the vertex ring domain of docked vacuoles. J Cell Biol, 167(6), 1087-1098. 
Fry, M. R., Thomson, J. M., Tomasini, A. J., \& Dunn Jr, W. A. (2006). Early and Late Molecular Events of Glucose-Induced Pexophagy in Pichia pastoris Require Vac8. Autophagy, 2(4), 280-288.

Fu, N., Yang, X., \& Chen, L. (2018). Nucleophagy Plays a Major Role in Human Diseases. Curr Drug Targets, 19(15), 1767-1773.

Fujioka, Y., Noda, N. N., Nakatogawa, H., Ohsumi, Y., \& Inagaki, F. (2010). Dimeric coiled-coil structure of Saccharomyces cerevisiae Atg16 and its functional significance in autophagy. J Biol Chem, 285(2), 1508-1515.

Fujioka, Y., Suzuki, S. W., Yamamoto, H., Kondo-Kakuta, C., Kimura, Y., Hirano, H., ... \& Noda, N. N. (2014). Structural basis of starvation-induced assembly of the autophagy initiation complex. Nat Struct Mol Biol, 21(6), 513.

Fujita, N., Hayashi-Nishino, M., Fukumoto, H., Omori, H., Yamamoto, A., Noda, T., \& Yoshimori, T. (2008). An Atg4B mutant hampers the lipidation of LC3 paralogues and causes defects in autophagosome closure. Mol Biol Cell, 19(11), 4651-4659.

Futter, C. E., Collinson, L. M., Backer, J. M., \& Hopkins, C. R. (2001). Human VPS34 is required for internal vesicle formation within multivesicular endosomes. J Cell Biol, 155(7), 1251-1264.

Gable, K., Garton, S., Napier, J. A., \& Dunn, T. M. (2004). Functional characterization of the Arabidopsis thaliana orthologue of Tsc13p, the enoyl reductase of the yeast microsomal fatty acid elongating system. J Exp Bot, 55(396), 543-545.

Gao, J., Langemeyer, L., Kümmel, D., Reggiori, F., \& Ungermann, C. (2018a). Molecular mechanism to target the endosomal Mon1-Ccz1 GEF complex to the pre-autophagosomal structure. Elife, 7, 31145.

Gao, J., Reggiori, F., \& Ungermann, C. (2018b). A novel in vitro assay reveals SNARE topology and the role of Ykt6 in autophagosome fusion with vacuoles. J Cell Biol, 217(10), 3670-3682.

Gasch, A. P., Spellman, P. T., Kao, C. M., Carmel-Harel, O., Eisen, M. B., Storz, G., .. \& Brown, P. O. (2000). Genomic expression programs in the response of yeast cells to environmental changes. Mol Biol Cell, 11(12), 4241-4257.

Gatica, D., Lahiri, V., \& Klionsky, D. J. (2018). Cargo recognition and degradation by selective autophagy. Nat Cell Biol, 20(3), 233.

Gavin, A. C., Bösche, M., Krause, R., Grandi, P., Marzioch, M., Bauer, A., ... \& Remor, M. (2002). Functional organization of the yeast proteome by systematic analysis of protein complexes. Nature, 415(6868), 141

Ge, L., Baskaran, S., Schekman, R., \& Hurley, J. H. (2014). The protein-vesicle network of autophagy. Curr Opin Cell Biol, 29, 18-24.

Gelperin, D. M., White, M. A., Wilkinson, M. L., Kon, Y., Kung, L. A., Wise, K. J., ... \& Gerstein, M. (2005). Biochemical and genetic analysis of the yeast proteome with a movable ORF collection. Genes Dev, 19(23), 2816-2826. 
Giaever, G., Chu, A. M., Ni, L., Connelly, C., Riles, L., Veronneau, S., .. \& Arkin, A. P. (2002). Functional profiling of the Saccharomyces cerevisiae genome. Nature, 418(6896), 387.

Gietz, R. D., \& Schiestl, R. H. (2007). High-efficiency yeast transformation using the LiAc/SS carrier DNA/PEG method. Nature Protoc, 2(1), 31.

Goffeau, A., Barrell, B. G., Bussey, H., Davis, R. W., Dujon, B., Feldmann, H., ... \& Louis, E. J. (1996). Life with 6000 genes. Science, 274(5287), 546-567.

Graef, M., Friedman, J. R., Graham, C., Babu, M., \& Nunnari, J. (2013). ER exit sites are physical and functional core autophagosome biogenesis components. Mol Biol Cell, 24(18), 2918-2931.

Guan, J., Stromhaug, P. E., George, M. D., Habibzadegah-Tari, P., Bevan, A., Dunn Jr, W. A., \& Klionsky, D. J. (2001). Cvt18/Gsa12 is required for cytoplasm-to-vacuole transport, pexophagy, and autophagy in Saccharomyces cerevisiae and Pichia pastoris. Mol Biol Cell, 12(12), 3821-3838.

Habibzadegah-Tari, P., \& Dunn Jr, W. A. (2003). Glucose-induced pexophagy in Pichia pastoris. Autophagy, ed. DJ Klionsky, Georgetown, TX: Landes Bioscience.

Hailey, D. W., Rambold, A. S., Satpute-Krishnan, P., Mitra, K., Sougrat, R., Kim, P. K., \& LippincottSchwartz, J. (2010). Mitochondria supply membranes for autophagosome biogenesis during starvation. Cell, 141(4), 656-667.

Hamasaki, M., Noda, T., Baba, M., \& Ohsumi, Y. (2005). Starvation triggers the delivery of the endoplasmic reticulum to the vacuole via autophagy in yeast. Traffic, 6(1), 56-65.

Han, G., Gable, K., Kohlwein, S. D., Beaudoin, F., Napier, J. A., \& Dunn, T. M. (2002). The Saccharomyces cerevisiae YBR159w gene encodes the 3-ketoreductase of the microsomal fatty acid elongase. J Biol Chem, 277(38), 35440-35449.

Hanada, T., Satomi, Y., Takao, T., \& Ohsumi, Y. (2009). The amino-terminal region of Atg3 is essential for association with phosphatidylethanolamine in Atg8 lipidation. FEBS Lett, 583(7), 1078-1083.

Hanada, T., Noda, N. N., Satomi, Y., Ichimura, Y., Fujioka, Y., Takao, T., ... \& Ohsumi, Y. (2007). The Atg12-Atg5 conjugate has a novel E3-like activity for protein lipidation in autophagy. J Biol Chem, 282(52), 37298-37302.

Hariri, H., Rogers, S., Ugrankar, R., Liu, Y. L., Feathers, J. R., \& Henne, W. M. (2018). Lipid droplet biogenesis is spatially coordinated at ER-vacuole contacts under nutritional stress. EMBO Rep, 19(1), 57-72.

Hariri, H., Speer, N., Bowerman, J., Rogers, S., Fu, G., Reetz, E., .. \& Henne, W. M. (2019). Mdm1 maintains endoplasmic reticulum homeostasis by spatially regulating lipid droplet biogenesis. $J$ Cell Biol, 218(4), 1319-1334.

Hatakeyama, R., \& De Virgilio, C. (2019). TORC1 specifically inhibits microautophagy through ESCRT-0. Genet, 1-7. 
Hayashi-Nishino, M., Fujita, N., Noda, T., Yamaguchi, A., Yoshimori, T., \& Yamamoto, A. (2009). A subdomain of the endoplasmic reticulum forms a cradle for autophagosome formation. Nat Cell Biol, 11(12), 1433.

He, C., Baba, M., Cao, Y., \& Klionsky, D. J. (2008). Self-interaction is critical for Atg9 transport and function at the phagophore assembly site during autophagy. Mol Biol Cell, 19(12), 5506-5516.

Heilmann, M., Iven, T., Ahmann, K., Hornung, E., Stymne, S., \& Feussner, I. (2012). Production of wax esters in plant seed oils by oleosomal cotargeting of biosynthetic enzymes. J Lipid Res, 53(10), 2153-2161.

Henne, W. M., Zhu, L., Balogi, Z., Stefan, C., Pleiss, J. A., \& Emr, S. D. (2015). Mdm1/Snx13 is a novel ER-endolysosomal interorganelle tethering protein. J Cell Biol, 210(4), 541-551.

Herman, P. K., Stack, J. H., \& Emr, S. D. (1991). A genetic and structural analysis of the yeast Vps15 protein kinase: evidence for a direct role of $\mathrm{Vps} 15 \mathrm{p}$ in vacuolar protein delivery. EMBO J, 10(13), 4049-4060.

Herrmann, T., van der Hoeven, F., Gröne, H. J., Stewart, A. F., Langbein, L., Kaiser, I., ... \& Schmitz, G. (2003). Mice with targeted disruption of the fatty acid transport protein 4 (Fatp 4, Slc27a4) gene show features of lethal restrictive dermopathy. J Cell Biol, 161(6), 1105-1115.

Herskowitz, I. (1988). Life cycle of the budding yeast Saccharomyces cerevisiae. Microbiological reviews, 52(4), 536.

Hickey, C. M., \& Wickner, W. (2010). HOPS initiates vacuole docking by tethering membranes before trans-SNARE complex assembly. Mol Biol Cell, 21(13), 2297-2305.

Hinnen, A., Hicks, J. B., \& Fink, G. R. (1978). Transformation of yeast. Proc Natl Acad Sci U.S.A, 75(4), 1929-1933.

Hou, H., Subramanian, K., LaGrassa, T. J., Markgraf, D., Dietrich, L. E., Urban, J., ... \& Ungermann, C. (2005). The DHHC protein Pfa3 affects vacuole-associated palmitoylation of the fusion factor Vac8. Proc Natl Acad Sci U.S.A, 102(48), 17366-17371.

Huang, R., \& Liu, W. (2015). Identifying an essential role of nuclear LC3 for autophagy. Autophagy, 11(5), 852-853.

Huang, R., Xu, Y., Wan, W., Shou, X., Qian, J., You, Z., ... \& Liu, W. (2015). Deacetylation of nuclear LC3 drives autophagy initiation under starvation. Mol Cell, 57(3), 456-466.

Hughes, T. R., Robinson, M. D., Mitsakakis, N., \& Johnston, M. (2004). The promise of functional genomics: completing the encyclopedia of a cell. Curr Opin Microbiol, 7(5), 546-554.

Huh, W. K., Falvo, J. V., Gerke, L. C., Carroll, A. S., Howson, R. W., Weissman, J. S., \& O'shea, E. K. (2003). Global analysis of protein localization in budding yeast. Nature, 425(6959), 686.

Ichimura, Y., Imamura, Y., Emoto, K., Umeda, M., Noda, T., \& Ohsumi, Y. (2004). In vivo and in vitro reconstitution of Atg8 conjugation essential for autophagy. J Biol Chem, 279(39), 40584-40592.

Ichimura, Y., Kirisako, T., Takao, T., Satomi, Y., Shimonishi, Y., Ishihara, N., ... \& Noda, T. (2000). A ubiquitin-like system mediates protein lipidation. Nature, 408(6811), 488. 
Ishihara, N., Hamasaki, M., Yokota, S., Suzuki, K., Kamada, Y., Kihara, A., .. \& Ohsumi, Y. (2001). Autophagosome requires specific early Sec proteins for its formation and NSF/SNARE for vacuolar fusion. Mol Biol Cell, 12(11), 3690-3702.

Itakura, E., Kishi-Itakura, C., \& Mizushima, N. (2012). The hairpin-type tail-anchored SNARE syntaxin 17 targets to autophagosomes for fusion with endosomes/lysosomes. Cell, 151(6), 1256-1269.

Ivanov, A., Pawlikowski, J., Manoharan, I., van Tuyn, J., Nelson, D. M., Rai, T. S., ... \& Wu, H. (2013). Lysosome-mediated processing of chromatin in senescence. J Cell Biol, 202(1), 129-143.

Jacomin, A. C., Samavedam, S., Promponas, V., \& Nezis, I. P. (2016). iLIR database: A web resource for LIR motif-containing proteins in eukaryotes. Autophagy, 12(10), 1945-1953.

Janke, C., Magiera, M. M., Rathfelder, N., Taxis, C., Reber, S., Maekawa, H., ... \& Knop, M. (2004). A versatile toolbox for PCR-based tagging of yeast genes: new fluorescent proteins, more markers and promoter substitution cassettes. Yeast, 21(11), 947-962.

Jao, C. C., Ragusa, M. J., Stanley, R. E., \& Hurley, J. H. (2013). A HORMA domain in Atg13 mediates PI 3-kinase recruitment in autophagy. Proc Natl Acad Sci U.S.A, 110(14), 5486-5491.

Jeong, H., Park, J., Kim, H. I., Lee, M., Ko, Y. J., Lee, S., ... \& Lee, C. (2017). Mechanistic insight into the nucleus-vacuole junction based on the Vac8p-Nvj1p crystal structure. Proc Natl Acad Sci U.S.A, 114(23), 4539-4548.

Jiang, B., Brown, J. L., Sheraton, J., Fortin, N., \& Bussey, H. (1994). A new family of yeast genes implicated in ergosterol synthesis is related to the human oxysterol binding protein. Yeast, 10(3), 341-353.

Juris, L., Montino, M., Rube, P., Schlotterhose, P., Thumm, M., \& Krick, R. (2015). PI3P binding by Atg21 organises Atg8 lipidation. EMBO J, 34(7), 955-973.

Kabeya, Y., Kamada, Y., Baba, M., Takikawa, H., Sasaki, M., \& Ohsumi, Y. (2005). Atg17 functions in cooperation with Atg1 and Atg13 in yeast autophagy. Mol Biol Cell, 16(5), 2544-2553.

Kabeya, Y., Noda, N. N., Fujioka, Y., Suzuki, K., Inagaki, F., \& Ohsumi, Y. (2009). Characterization of the Atg17-Atg29-Atg31 complex specifically required for starvation-induced autophagy in Saccharomyces cerevisiae. Biochem Biophys Res Commun, 389(4), 612-615.

Kaeberlein, M. (2010). Lessons on longevity from budding yeast. Nature, 464(7288), 513.

Käll, L., Krogh, A., \& Sonnhammer, E. L. (2004). A combined transmembrane topology and signal peptide prediction method. J Mol Biol, 338(5), 1027-1036.

Kalvari, I., Tsompanis, S., Mulakkal, N. C., Osgood, R., Johansen, T., Nezis, I. P., \& Promponas, V. J. (2014). iLIR: A web resource for prediction of Atg8-family interacting proteins. Autophagy, 10(5), 913-925.

Kamada, Y., Funakoshi, T., Shintani, T., Nagano, K., Ohsumi, M., \& Ohsumi, Y. (2000). Tormediated induction of autophagy via an Apg1 protein kinase complex. J Cell Biol, 150(6), 1507-1513. 
Kamada, Y., Yoshino, K. I., Kondo, C., Kawamata, T., Oshiro, N., Yonezawa, K., \& Ohsumi, Y. (2010). Tor directly controls the Atg1 kinase complex to regulate autophagy. Mol Cell Biol, 30(4), 1049-1058.

Kaplan, C., \& Ewers, H. (2015). Optimized sample preparation for single-molecule localizationbased superresolution microscopy in yeast. Nat Protoc, 10(7), 1007.

Karantza-Wadsworth, V., Patel, S., Kravchuk, O., Chen, G., Mathew, R., Jin, S., \& White, E. (2007). Autophagy mitigates metabolic stress and genome damage in mammary tumorigenesis. Genes Dev, 21(13), 1621-1635.

Kaufmann, A., Beier, V., Franquelim, H. G., \& Wollert, T. (2014). Molecular mechanism of autophagic membrane-scaffold assembly and disassembly. Cell, 156(3), 469-481.

Kaushik, S., \& Cuervo, A. M. (2012). Chaperone-mediated autophagy: a unique way to enter the lysosome world. Trends Cell Biol, 22(8), 407-417.

Kawamata, T., Kamada, Y., Kabeya, Y., Sekito, T., \& Ohsumi, Y. (2008). Organization of the preautophagosomal structure responsible for autophagosome formation. Mol Biol Cell, 19(5), 2039-2050.

Khafif, M., Cottret, L., Balagué, C., \& Raffaele, S. (2014). Identification and phylogenetic analyses of VASt, an uncharacterized protein domain associated with lipid-binding domains in Eukaryotes. BMC Bioinform, 15(1), 222.

Kihara, A., Kabeya, Y., Ohsumi, Y., \& Yoshimori, T. (2001). Beclin-phosphatidylinositol 3-kinase complex functions at the trans-Golgi network. EMBO Rep, 2(4), 330-335.

Kimura, S., Noda, T., \& Yoshimori, T. (2008). Dynein-dependent movement of autophagosomes mediates efficient encounters with lysosomes. Cell Struct Funct, 33(1), 109-122.

Kira, S., Kumano, Y., Ukai, H., Takeda, E., Matsuura, A., \& Noda, T. (2016). Dynamic relocation of the TORC1-Gtr1/2-Ego1/2/3 complex is regulated by Gtr1 and Gtr2. Mol Biol Cell, 27(2), 382-396.

Kira, S., Tabata, K., Shirahama-Noda, K., Nozoe, A., Yoshimori, T., \& Noda, T. (2014). Reciprocal conversion of Gtr1 and Gtr2 nucleotide-binding states by Npr2-Npr3 inactivates TORC1 and induces autophagy. Autophagy, 10(9), 1565-1578.

Kirisako, T., Baba, M., Ishihara, N., Miyazawa, K., Ohsumi, M., Yoshimori, T., ... \& Ohsumi, Y. (1999). Formation process of autophagosome is traced with Apg8/Aut7p in yeast. J Cell Biol, 147(2), 435-446.

Kirisako, T., Ichimura, Y., Okada, H., Kabeya, Y., Mizushima, N., Yoshimori, T., ... \& Ohsumi, Y. (2000). The reversible modification regulates the membrane-binding state of Apg8/Aut7 essential for autophagy and the cytoplasm to vacuole targeting pathway. J Cell Biol, 151(2), 263-276.

Kiššová, I. B., Salin, B., Schaeffer, J., Bhatia, S., Manon, S., \& Camougrand, N. (2007). Selective and non-selective autophagic degradation of mitochondria in yeast. Autophagy, 3(4), 329-336. 
Klar, A. J. (1987). Differentiated parental DNA strands confer developmental asymmetry on daughter cells in fission yeast. Nature, 326(6112), 466.

Klionsky, D. J. (2005). The molecular machinery of autophagy: unanswered questions. J Cell Sci, 118(1), 7-18.

Klionsky, D. J., Abeliovich, H., Agostinis, P., Agrawal, D. K., Aliev, G., Askew, D. S., ... \& Bamber, B. A. (2008). Guidelines for the use and interpretation of assays for monitoring autophagy in higher eukaryotes. Autophagy, 4(2), 151-175.

Klionsky, D. J., \& Emr, S. D. (1989). Membrane protein sorting: biosynthesis, transport and processing of yeast vacuolar alkaline phosphatase. EMBO J, 8(8), 2241-2250.

Klionsky, D. J., Herman, P. K., \& Emr, S. D. (1990). The fungal vacuole: composition, function, and biogenesis. Microbiol Mol Biol Rev, 54(3), 266-292.

Knop, M., Schiffer, H. H., Rupp, S., \& Wolf, D. H. (1993). Vacuolar/lysosomal proteolysis: proteases, substrates mechanisms. Curr Opin Cell Biol, 5(6), 990-996.

Knop, M., Siegers, K., Pereira, G., Zachariae, W., Winsor, B., Nasmyth, K., \& Schiebel, E. (1999). Epitope tagging of yeast genes using a PCR-based strategy: more tags and improved practical routines. Yeast, 15(10), 963-972.

Köchl, R., Hu, X. W., Chan, E. Y., \& Tooze, S. A. (2006). Microtubules facilitate autophagosome formation and fusion of autophagosomes with endosomes. Traffic, 7(2), 129-145.

Kohlwein, S. D., Eder, S., Oh, C. S., Martin, C. E., Gable, K., Bacikova, D., \& Dunn, T. (2001). Tsc13p Is Required for Fatty Acid Elongation and Localizes to a Novel Structure at the NuclearVacuolar Interface in Saccharomyces cerevisiae. Mol Cell Biol, 21(1), 109-125.

Koizumi, K., \& Gallagher, K. L. (2013). Identification of SHRUBBY, a SHORT-ROOT and SCARECROW interacting protein that controls root growth and radial patterning. Development, 140(6), 1292-1300.

Kolehmainen, J., Black, G. C., Saarinen, A., Chandler, K., Clayton-Smith, J., Träskelin, A. L., ... \& Fryns, J. P. (2003). Cohen syndrome is caused by mutations in a novel gene, $\mathrm{COH} 1$, encoding a transmembrane protein with a presumed role in vesicle-mediated sorting and intracellular protein transport. Am J Hum Genet, 72(6), 1359-1369.

Kominami, E., Hoffschulte, H., \& Holzer, H. (1981). Purification and properties of proteinase B from yeast. Biochimica et Biophysica Acta (BBA)-Enzymology, 661(1), 124-135.

Kopec, K. O., Alva, V., \& Lupas, A. N. (2010). Homology of SMP domains to the TULIP superfamily of lipid-binding proteins provides a structural basis for lipid exchange between ER and mitochondria. Bioinformatics, 26(16), 1927-1931.

Kornmann, B., Currie, E., Collins, S. R., Schuldiner, M., Nunnari, J., Weissman, J. S., \& Walter, P. (2009). An ER-mitochondria tethering complex revealed by a synthetic biology screen. Science, 325(5939), 477-481. 
Krick, R., Mühe, Y., Prick, T., Bredschneider, M., Bremer, S., Wenzel, D., .. \& Thumm, M. (2009). Piecemeal microautophagy of the nucleus: genetic and morphological traits. Autophagy, 5(2), 270-272.

Krick, R., Mühe, Y., Prick, T., Bremer, S., Schlotterhose, P., Eskelinen, E. L., ... \& Thumm, M. (2008). Piecemeal microautophagy of the nucleus requires the core macroautophagy genes. Mol Biol Cell, 19(10), 4492-4505.

Kruppa, A. J., Kendrick-Jones, J., \& Buss, F. (2016). Myosins, actin and autophagy. Traffic, 17(8), 878-890.

Kuma, A., Matsui, M., \& Mizushima, N. (2007). LC3, an autophagosome marker, can be incorporated into protein aggregates independent of autophagy: caution in the interpretation of LC3 localization. Autophagy, 3(4), 323-328.

Kuma, A., Mizushima, N., Ishihara, N., \& Ohsumi, Y. (2002). Formation of the $350-k D a$ Apg12Apg5.Apg 16 multimeric complex, mediated by Apg16 oligomerization, is essential for autophagy in yeast. J Biol Chem, 277(21), 18619-18625.

Kvam, E., Gable, K., Dunn, T. M., \& Goldfarb, D. S. (2005). Targeting of Tsc13p to nucleus-vacuole junctions: a role for very-long-chain fatty acids in the biogenesis of microautophagic vesicles. Mol Biol Cell, 16(9), 3987-3998.

Kvam, E., \& Goldfarb, D. S. (2004). Nvj1p is the outer-nuclear-membrane receptor for oxysterolbinding protein homolog Osh1p in Saccharomyces cerevisiae. J Cell Sci, 117(21), 4959-4968.

Kvam, E., \& Goldfarb, D. S. (2007). Nucleus-vacuole junctions and piecemeal microautophagy of the nucleus in S. cerevisiae. Autophagy, 3(2), 85-92.

Laemmli, U. K. (1970). Cleavage of structural proteins during the assembly of the head of bacteriophage T4. Nature, 227(5259), 680.

Landajuela, A., Hervás, J. H., Antón, Z., Montes, L. R., Gil, D., Valle, M., ... \& Alonso, A. (2016). Lipid geometry and bilayer curvature modulate LC3/GABARAP-mediated model autophagosomal elongation. Biophys J, 110(2), 411-422.

Lang, A. B., Peter, A. T. J., Walter, P., \& Kornmann, B. (2015). ER-mitochondrial junctions can be bypassed by dominant mutations in the endosomal protein Vps13. J Cell Biol, 210(6), 883-890.

Lang, T., Reiche, S., Straub, M., Bredschneider, M., \& Thumm, M. (2000). Autophagy and the cvt Pathway Both Depend on AUT9. J Bacteriol, 182(8), 2125-2133.

Lee, I., \& Hong, W. (2006). Diverse membrane-associated proteins contain a novel SMP domain. FASEB J, 20(2), 202-206.

Lee, I. H., Kawai, Y., Fergusson, M. M., Rovira, I. I., Bishop, A. J., Motoyama, N., .. \& Finkel, T. (2012). Atg7 modulates p53 activity to regulate cell cycle and survival during metabolic stress. Science, 336(6078), 225-228.

Lehto, M., Laitinen, S., Chinetti, G., Johansson, M., Ehnholm, C., Staels, B., .. \& Olkkonen, V. M. (2001). The OSBP-related protein family in humans. J Lipid Res, 42(8), 1203-1213. 
Lemus, L., Ribas, J. L., Sikorska, N., \& Goder, V. (2016). An ER-localized SNARE protein is exported in specific COPII vesicles for autophagosome biogenesis. Cell Rep, 14(7), 1710-1722.

Lenney, J. F., Matile, P., Wiemken, A., Schellenberg, M., \& Meyer, J. (1974). Activities and cellular localization of yeast proteases and their inhibitors. Biochem Biophys Res Commun, 60(4), 1378-1383.

Lesage, S., Drouet, V., Majounie, E., Deramecourt, V., Jacoupy, M., Nicolas, A., ... \& Erpapazoglou, Z. (2016). Loss of VPS13C function in autosomal-recessive Parkinsonism causes mitochondrial dysfunction and increases PINK1/Parkin-dependent mitophagy. Am J Hum Genet, 98(3), 500-513.

Levental, I., Lingwood, D., Grzybek, M., Coskun, Ü., \& Simons, K. (2010). Palmitoylation regulates raft affinity for the majority of integral raft proteins. Proc Natl Acad Sci U.S.A, 107(51), 22050-22054.

Levine, B., \& Klionsky, D. J. (2004). Development by self-digestion: molecular mechanisms and biological functions of autophagy. Dev Cell, 6(4), 463-477.

Levine, T. P., \& Munro, S. (2001). Dual targeting of Osh1p, a yeast homologue of oxysterol-binding protein, to both the Golgi and the nucleus-vacuole junction. Mol Biol Cell, 12(6), 1633-1644.

Li, W. W., Li, J., \& Bao, J. K. (2012). Microautophagy: lesser-known self-eating. Cell Mol Life Sci, 69(7), 1125-1136.

Liang, X. H., Jackson, S., Seaman, M., Brown, K., Kempkes, B., Hibshoosh, H., \& Levine, B. (1999). Induction of autophagy and inhibition of tumorigenesis by beclin 1. Nature, 402(6762), 672.

Liu, X., \& Klionsky, D. J. (2016). The Atg17-Atg31-Atg29 complex and Atg11 regulate autophagosome-vacuole fusion. Autophagy, 12(5), 894-895.

Liu, X., Mao, K., Angela, Y. H., Omairi-Nasser, A., Austin II, J., Glick, B. S., ... \& Klionsky, D. J. (2016). The Atg17-Atg31-Atg29 complex coordinates with Atg11 to recruit the Vam7 SNARE and mediate autophagosome-vacuole fusion. Curr Biol, 26(2), 150-160.

Loewen, C. J., Roy, A., \& Levine, T. P. (2003). A conserved ER targeting motif in three families of lipid binding proteins and in Opi1p binds VAP. EMBO J, 22(9), 2025-2035.

Longtine, M. S., Mckenzie III, A., Demarini, D. J., Shah, N. G., Wach, A., Brachat, A., ... \& Pringle, J. R. (1998). Additional modules for versatile and economical PCR-based gene deletion and modification in Saccharomyces cerevisiae. Yeast, 14(10), 953-961.

Luzhna, L., Kathiria, P., \& Kovalchuk, O. (2013). Micronuclei in genotoxicity assessment: from genetics to epigenetics and beyond. Front Genet, 4, 131.

Lv, L., Li, D., Zhao, D., Lin, R., Chu, Y., Zhang, H., .. \& Wang, G. (2011). Acetylation targets the M2 isoform of pyruvate kinase for degradation through chaperone-mediated autophagy and promotes tumor growth. Mol Cell, 42(6), 719-730.

Lynch-Day, M. A., Bhandari, D., Menon, S., Huang, J., Cai, H., Bartholomew, C. R., ... \& Klionsky, D. J. (2010). Trs85 directs a Ypt1 GEF, TRAPPIII, to the phagophore to promote autophagy. Proc Natl Acad Sci U.S.A, 107(17), 7811-7816. 
Mager, W. H., \& Winderickx, J. (2005). Yeast as a model for medical and medicinal research. Trends Pharmacol Sci, 26(5), 265-273.

Mandel, M., \& Higa, A. (1970). Calcium-dependent bacteriophage DNA infection. J Mol Biol, $53(1), 159-162$.

Manford, A. G., Stefan, C. J., Yuan, H. L., MacGurn, J. A., \& Emr, S. D. (2012). ER-to-plasma membrane tethering proteins regulate cell signaling and ER morphology. Dev Cell, 23(6), 1129-1140.

Manik, M. K., Yang, H., Tong, J., \& Im, Y. J. (2017). Structure of yeast OSBP-related protein Osh1 reveals key determinants for lipid transport and protein targeting at the nucleus-vacuole junction. Structure, 25(4), 617-629.

Manivannan, S., de Boer, R., Veenhuis, M., \& van der Klei, I. J. (2013). Lumenal peroxisomal protein aggregates are removed by concerted fission and autophagy events. Autophagy, 9(7), 1044-1056.

Mao, K., Chew, L. H., Inoue-Aono, Y., Cheong, H., Nair, U., Popelka, H., ... \& Klionsky, D. J. (2013). Atg29 phosphorylation regulates coordination of the Atg17-Atg31-Atg29 complex with the Atg11 scaffold during autophagy initiation. Proc Natl Acad Sci U.S.A, 110(31), 2875-2884.

Mao, K., Liu, X., Feng, Y., \& Klionsky, D. J. (2014). The progression of peroxisomal degradation through autophagy requires peroxisomal division. Autophagy, 10(4), 652-661.

Mari, M., Griffith, J., Rieter, E., Krishnappa, L., Klionsky, D. J., \& Reggiori, F. (2010). An Atg9containing compartment that functions in the early steps of autophagosome biogenesis. J Cell Biol, 190(6), 1005-1022.

Matsuura, A., Tsukada, M., Wada, Y., \& Ohsumi, Y. (1997). Apg1p, a novel protein kinase required for the autophagic process in Saccharomyces cerevisiae. Gene, 192(2), 245-250.

McMillan, J., Lu, Z., Rodriguez, J. S., Ahn, T. H., \& Lin, Z. (2019). YeasTSS: an integrative web database of yeast transcription start sites. Database, in press.

Meiling-Wesse, K., Barth, H., \& Thumm, M. (2002). Ccz1p/Aut11p/Cvt16p is essential for autophagy and the cvt pathway. FEBS Lett, 526(1-3), 71-76.

Merzlyak, E. M., Goedhart, J., Shcherbo, D., Bulina, M. E., Shcheglov, A. S., Fradkov, A. F., ... \& Chudakov, D. M. (2007). Bright monomeric red fluorescent protein with an extended fluorescence lifetime. Nat Methods, 4(7), 555.

Mesmin, B., \& Antonny, B. (2016). The counterflow transport of sterols and PI4P. Biochimica et Biophysica Acta (BBA)-Molecular and Cell Biology of Lipids, 1861(8), 940-951.

Mesmin, B., Bigay, J., von Filseck, J. M., Lacas-Gervais, S., Drin, G., \& Antonny, B. (2013). A fourstep cycle driven by PI (4) P hydrolysis directs sterol/PI (4) P exchange by the ER-Golgi tether OSBP. Cell, 155(4), 830-843.

Mewes, H., Albermann, K., Bähr, M., Frishman, D., Gleissner, A., Hani, J., ... \& Pfeiffer, F. (1997). Overview of the yeast genome. Nature, 387(6632), 7-8. 
Mijaljica, D., Nazarko, T. Y., Brumell, J. H., Huang, W. P., Komatsu, M., Prescott, M., ... \& Devenish, R. J. (2012). Receptor protein complexes are in control of autophagy. Autophagy, 8(11), 1701-1705.

Mijaljica, D., Prescott, M., \& Devenish, R. J. (2010). The intricacy of nuclear membrane dynamics during nucleophagy. Nucleus, 1(3), 213-223.

Millen, J. I., Krick, R., Prick, T., Thumm, M., \& Goldfarb, D. S. (2009). Measuring piecemeal microautophagy of the nucleus in Saccharomyces cerevisiae. Autophagy, 5(1), 75-81.

Millen, J. I., Pierson, J., Kvam, E., Olsen, L. J., \& Goldfarb, D. S. (2008). The luminal N-terminus of yeast Nvj1 is an inner nuclear membrane anchor. Traffic, 9(10), 1653-1664.

Mizushima, N., \& Levine, B. (2010). Autophagy in mammalian development and differentiation. Nat Cell Biol, 12(9), 823.

Mizushima, N., Noda, T., \& Ohsumi, Y. (1999). Apg16p is required for the function of the Apg12pApg5p conjugate in the yeast autophagy pathway. EMBO J, 18(14), 3888-3896.

Mizushima, N., Yoshimori, T., \& Ohsumi, Y. (2011). The role of Atg proteins in autophagosome formation. Annu Rev Cell Dev Biol, 27, 107-132.

Mochida, K., Ohsumi, Y., \& Nakatogawa, H. (2014). Hrr25 phosphorylates the autophagic receptor Atg34 to promote vacuolar transport of $\alpha$-mannosidase under nitrogen starvation conditions. FEBS Lett, 588(21), 3862-3869.

Mochida, K., Oikawa, Y., Kimura, Y., Kirisako, H., Hirano, H., Ohsumi, Y., \& Nakatogawa, H. (2015). Receptor-mediated selective autophagy degrades the endoplasmic reticulum and the nucleus. Nature, 522(7556), 359.

Moehle, C. M., Tizard, R., Lemmon, S. K., Smart, J., \& Jones, E. W. (1987). Protease B of the lysosomelike vacuole of the yeast Saccharomyces cerevisiae is homologous to the subtilisin family of serine proteases. Mol Cell Biol, 7(12), 4390-4399.

Moser von Filseck, J., Čopič, A., Delfosse, V., Vanni, S., Jackson, C. L., Bourguet, W., \& Drin, G. (2015). Phosphatidylserine transport by ORP/Osh proteins is driven by phosphatidylinositol 4phosphate. Science, 349(6246), 432-436.

Moskvina, E., Schüller, C., Maurer, C. T. C., Mager, W. H., \& Ruis, H. (1998). A search in the genome of Saccharomyces cerevisiae for genes regulated via stress response elements. Yeast, 14(11), 1041-1050.

Mostofa, M. G., Rahman, M. A., Koike, N., Yeasmin, A. M., Islam, N., Waliullah, T. M., .. \& Ushimaru, T. (2018). CLIP and cohibin separate rDNA from nucleolar proteins destined for degradation by nucleophagy. J Cell Biol, 217(8), 2675-2690.

Motley, A. M., Nuttall, J. M., \& Hettema, E. H. (2012). Pex3-anchored Atg36 tags peroxisomes for degradation in Saccharomyces cerevisiae. EMBO J, 31(13), 2852-2868. 
Mukaiyama, H., Baba, M., Osumi, M., Aoyagi, S., Kato, N., Ohsumi, Y., \& Sakai, Y. (2004). Modification of a ubiquitin-like protein Paz2 conducted micropexophagy through formation of a novel membrane structure. Mol Biol Cell, 15(1), 58-70.

Müller, O., Sattler, T., Flötenmeyer, M., Schwarz, H., Plattner, H., \& Mayer, A. (2000). Autophagic tubes: vacuolar invaginations involved in lateral membrane sorting and inverse vesicle budding. $J$ Cell Biol, 151(3), 519-528.

Muñoz-Braceras, S., Calvo, R., \& Escalante, R. (2015). TipC and the chorea-acanthocytosis protein VPS13A regulate autophagy in Dictyostelium and human HeLa cells. Autophagy, 11(6), 918-927.

Murley, A., Sarsam, R. D., Toulmay, A., Yamada, J., Prinz, W. A., \& Nunnari, J. (2015). Ltc1 is an ERlocalized sterol transporter and a component of ER-mitochondria and ER-vacuole contacts. J Cell Biol, 209(4), 539-548.

Nadolski, M. J., \& Linder, M. E. (2009). Molecular recognition of the palmitoylation substrate Vac8 by its palmitoyltransferase Pfa3. J Biol Chem, 284(26), 17720-17730.

Nair, U., Jotwani, A., Geng, J., Gammoh, N., Richerson, D., Yen, W. L., ... \& Baba, M. (2011). SNARE proteins are required for macroautophagy. Cell, 146(2), 290-302.

Nakamura, N., Matsuura, A., Wada, Y., \& Ohsumi, Y. (1997). Acidification of vacuoles is required for autophagic degradation in the yeast, Saccharomyces cerevisiae. J Biochem, 121(2), 338-344.

Nakatogawa, H., Ichimura, Y., \& Ohsumi, Y. (2007). Atg8, a ubiquitin-like protein required for autophagosome formation, mediates membrane tethering and hemifusion. Cell, 130(1), 165-178.

Nath, S., Dancourt, J., Shteyn, V., Puente, G., Fong, W. M., Nag, S., ... \& Melia, T. J. (2014). Lipidation of the LC3/GABARAP family of autophagy proteins relies on a membrane-curvaturesensing domain in Atg3. Nat Cell Biol, 16(5), 415.

Nazarko, V. Y., Nazarko, T. Y., Farre, J. C., Stasyk, O. V., Warnecke, D., Ulaszewski, S., ... \& Subramani, S. (2011). Atg35, a micropexophagy-specific protein that regulates micropexophagic apparatus formation in Pichia pastoris. Autophagy, 7(4), 375-385.

Nazarko, T. Y., Ozeki, K., Till, A., Ramakrishnan, G., Lotfi, P., Yan, M., \& Subramani, S. (2014). Peroxisomal Atg37 binds Atg30 or palmitoyl-CoA to regulate phagophore formation during pexophagy. J Cell Biol, 204(4), 541-557.

Ngo, M., \& Ridgway, N. D. (2009). Oxysterol binding protein-related protein 9 (ORP9) is a cholesterol transfer protein that regulates Golgi structure and function. Mol Biol Cell, 20(5), 1388-1399.

Nguyen, T. T., Lewandowska, A., Choi, J. Y., Markgraf, D. F., Junker, M., Bilgin, M., ... \& Shaw, J. M. (2012). Gem1 and ERMES do not directly affect phosphatidylserine transport from ER to mitochondria or mitochondrial inheritance. Traffic, 13(6), 880-890.

Niedenthal, R. K., Riles, L., Johnston, M., \& Hegemann, J. H. (1996). Green fluorescent protein as a marker for gene expression and subcellular localization in budding yeast. Yeast, 12(8), 773-786. 
Noda, N. N., Kumeta, H., Nakatogawa, H., Satoo, K., Adachi, W., Ishii, J., ... \& Inagaki, F. (2008). Structural basis of target recognition by Atg8/LC3 during selective autophagy. Genes Cells, 13(12), 1211-1218.

Noda, T., Kim, J., Huang, W. P., Baba, M., Tokunaga, C., Ohsumi, Y., \& Klionsky, D. J. (2000). Apg9p/Cvt7p is an integral membrane protein required for transport vesicle formation in the Cvt and autophagy pathways. J Cell Biol, 148(3), 465-480.

Noda, T., \& Ohsumi, Y. (1998). Tor, a phosphatidylinositol kinase homologue, controls autophagy in yeast. J Biol Chem, 273(7), 3963-3966.

Nordmann, M., Cabrera, M., Perz, A., Bröcker, C., Ostrowicz, C., Engelbrecht-Vandré, S., \& Ungermann, C. (2010). The Mon1-Ccz1 complex is the GEF of the late endosomal Rab7 homolog Ypt7. Curr Biol, 20(18), 1654-1659.

Obara, K., Sekito, T., \& Ohsumi, Y. (2006). Assortment of phosphatidylinositol 3-kinase complexes -Atg14p directs association of complex I to the pre-autophagosomal structure in Saccharomyces cerevisiae. Mol Biol Cell, 17(4), 1527-1539.

Ohashi, Y., Soler, N., García Ortegón, M., Zhang, L., Kirsten, M. L., Perisic, O., ... \& Johnson, C. M. (2016). Characterization of Atg38 and NRBF2, a fifth subunit of the autophagic Vps34/PIK3C3 complex. Autophagy, 12(11), 2129-2144.

Oku, M., Nishimura, T., Hattori, T., Ano, Y., Yamashita, S. I., \& Sakai, Y. (2006). Role of Vac8 in formation of the vacuolar sequestering membrane during micropexophagy. Autophagy, 2(4), 272-279.

Oku, M., Warnecke, D., Noda, T., Müller, F., Heinz, E., Mukaiyama, H., .. \& Sakai, Y. (2003). Peroxisome degradation requires catalytically active sterol glucosyltransferase with a GRAM domain. EMBO J, 22(13), 3231-3241.

Pan, X., Roberts, P., Chen, Y., Kvam, E., Shulga, N., Huang, K., .. \& Goldfarb, D. S. (2000). Nucleus-vacuole junctions in Saccharomyces cerevisiae are formed through the direct interaction of Vac8p with Nvj1p. Mol Biol Cell, 11(7), 2445-2457.

Papandreou, M. E., \& Tavernarakis, N. (2019). Nucleophagy: from homeostasis to disease. Cell Death Differ, 1.

Papinski, D., Schuschnig, M., Reiter, W., Wilhelm, L., Barnes, C. A., Maiolica, A., ... \& Lee, S. S. (2014). Early steps in autophagy depend on direct phosphorylation of Atg9 by the Atg1 kinase. Mol Cell, 53(3), 471-483.

Park, C., Suh, Y., \& Cuervo, A. M. (2015). Regulated degradation of Chk1 by chaperone-mediated autophagy in response to DNA damage. Nat Commun, 6, 6823.

Park, H. K., Chu, K., Jung, K. H., Lee, S. T., Bahn, J. J., Kim, M., ... \& Roh, J. K. (2009). Autophagy is involved in the ischemic preconditioning. Neurosci Lett, 451(1), 16-19.

Park, J. S., Okumura, Y., Tachikawa, H., \& Neiman, A. M. (2013). SPO71 encodes a developmental stage-specific partner for Vps13 in Saccharomyces cerevisiae. Eukaryot Cell, 12(11), 1530-1537. 
Park, J. S., Thorsness, M. K., Policastro, R., McGoldrick, L. L., Hollingsworth, N. M., Thorsness, P. E., \& Neiman, A. M. (2016). Yeast Vps13 promotes mitochondrial function and is localized at membrane contact sites. Mol Biol Cell, 27(15), 2435-2449.

Parrish, W. R., Stefan, C. J., \& Emr, S. D. (2004). Essential role for the myotubularin-related phosphatase Ymr1p and the synaptojanin-like phosphatases Sjl2p and Sjl3p in regulation of phosphatidylinositol 3-phosphate in yeast. Mol Biol Cell, 15(8), 3567-3579.

Partow, S., Siewers, V., Bjørn, S., Nielsen, J., \& Maury, J. (2010). Characterization of different promoters for designing a new expression vector in Saccharomyces cerevisiae. Yeast, 27(11), 955-964.

Parzych, K. R., Ariosa, A., Mari, M., \& Klionsky, D. J. (2018). A newly characterized vacuolar serine carboxypeptidase, Atg42/Ybr139w, is required for normal vacuole function and the terminal steps of autophagy in the yeast Saccharomyces cerevisiae. Mol Biol Cell, 29(9), 1089-1099.

Peng, Y., Tang, F., \& Weisman, L. S. (2006). Palmitoylation plays a role in targeting Vac8p to specific membrane subdomains. Traffic, 7(10), 1378-1387.

Petrozzi, L., Lucetti, C., Scarpato, R., Gambaccini, G., Trippi, F., Bernardini, S., .. \& Bonuccelli, U. (2002). Cytogenetic alterations in lymphocytes of Alzheimer's disease and Parkinson's disease patients. Neurol Sci, 23(2), s97-s98.

Pfaffenwimmer, T., Reiter, W., Brach, T., Nogellova, V., Papinski, D., Schuschnig, M., ... \& Kraft, C. (2014). Hrr25 kinase promotes selective autophagy by phosphorylating the cargo receptor Atg19. EMBO Rep, 15(8), 862-870.

Popelka, H., Damasio, A., Hinshaw, J. E., Klionsky, D. J., \& Ragusa, M. J. (2017). Structure and function of yeast Atg20, a sorting nexin that facilitates autophagy induction. Proc Natl Acad Sci U.S.A, 114(47), 10112-10121.

Prosser, D. C., Tran, D., Gougeon, P. Y., Verly, C., \& Ngsee, J. K. (2008). FFAT rescues VAPAmediated inhibition of ER-to-Golgi transport and VAPB-mediated ER aggregation. J Cell Sci, 121(18), 3052-3061.

Qu, X., Yu, J., Bhagat, G., Furuya, N., Hibshoosh, H., Troxel, A., ... \& Cattoretti, G. (2003). Promotion of tumorigenesis by heterozygous disruption of the beclin 1 autophagy gene. Journal Clin Invest, 112(12), 1809-1820.

Ragusa, M. J., Stanley, R. E., \& Hurley, J. H. (2012). Architecture of the Atg17 complex as a scaffold for autophagosome biogenesis. Cell, 151(7), 1501-1512.

Rahman, M. A., Mostofa, M. G., \& Ushimaru, T. (2018). The Nem1/Spo7-Pah1/lipin axis is required for autophagy induction after TORC 1 inactivation. FEBS J, 285(10), 1840-1860.

Rampoldi, L., Dobson-Stone, C., Rubio, J. P., Danek, A., Chalmers, R. M., Wood, N. W., ... \& Brown, R. (2001). A conserved sorting-associated protein is mutant in chorea-acanthocytosis. Nat Genet, 28(2), 119. 
Ramya, V., \& Rajasekharan, R. (2016). ATG 15 encodes a phospholipase and is transcriptionally regulated by YAP 1 in Saccharomyces cerevisiae. FEBS Lett, 590(18), 3155-3167.

Rao, Y., Perna, M. G., Hofmann, B., Beier, V., \& Wollert, T. (2016). The Atg1-kinase complex tethers Atg9-vesicles to initiate autophagy. Nat Commun, 7, 10338.

Ravikumar, B., Acevedo-Arozena, A., Imarisio, S., Berger, Z., Vacher, C., O'Kane, C. J., ... \& Rubinsztein, D. C. (2005). Dynein mutations impair autophagic clearance of aggregate-prone proteins. Nature Genet, 37(7), 771.

Raychaudhuri, S., \& Prinz, W. A. (2010). The diverse functions of oxysterol-binding proteins. Annu Rev Cell Dev Biol, 26, 157-177.

Reaves, B. J., Bright, N. A., Mullock, B. M., \& Luzio, J. P. (1996). The effect of wortmannin on the localisation of lysosomal type I integral membrane glycoproteins suggests a role for phosphoinositide 3-kinase activity in regulating membrane traffic late in the endocytic pathway. $J$ Cell Sci, 109(4), 749-762.

Reggiori, F., \& Klionsky, D. J. (2002). Autophagy in the eukaryotic cell. Eukaryot Cell, 1(1), 11-21.

Reggiori, F., Monastyrska, I., Shintani, T., \& Klionsky, D. J. (2005). The actin cytoskeleton is required for selective types of autophagy, but not nonspecific autophagy, in the yeast Saccharomyces cerevisiae. Mol Biol Cell, 16(12), 5843-5856.

Reggiori, F., Tucker, K. A., Stromhaug, P. E., \& Klionsky, D. J. (2004). The Atg1-Atg13 complex regulates Atg9 and Atg23 retrieval transport from the pre-autophagosomal structure. Dev Cell, 6(1), 79-90.

Reidick, C., Boutouja, F., \& Platta, H. W. (2017). The class III phosphatidylinositol 3-kinase Vps34 in Saccharomyces cerevisiae. Biol Chem, 398(5-6), 677-685.

Rello-Varona, S., Lissa, D., Shen, S., Niso-Santano, M., Senovilla, L., Mariño, G., ... \& Castedo, M. (2012). Autophagic removal of micronuclei. Cell Cycle, 11(1), 170-176.

Ries, J., Kaplan, C., Platonova, E., Eghlidi, H., \& Ewers, H. (2012). A simple, versatile method for GFP-based super-resolution microscopy via nanobodies. Nat Methods, 9(6), 582.

Roberts, P., Moshitch-Moshkovitz, S., Kvam, E., O'Toole, E., Winey, M., \& Goldfarb, D. S. (2003). Piecemeal microautophagy of nucleus in Saccharomyces cerevisiae. Mol Biol Cell, 14(1), 129-141.

Rogov, V., Dötsch, V., Johansen, T., \& Kirkin, V. (2014). Interactions between autophagy receptors and ubiquitin-like proteins form the molecular basis for selective autophagy. Mol Cell, 53(2), 167-178.

Rueden, C. T., Schindelin, J., Hiner, M. C., DeZonia, B. E., Walter, A. E., Arena, E. T., \& Eliceiri, K. W. (2017). ImageJ2: ImageJ for the next generation of scientific image data. BMC Bioinform, 18(1), 529.

Rupp, S., Hirsch, H. H., \& Wolf, D. H. (1991). Biogenesis of the yeast vacuole (lysosome) Active site mutation in the vacuolar aspartate proteinase yscA blocks maturation of vacuolar proteinases. FEBS Lett, 293(1-2), 62-66. 
Rzepnikowska, W., Flis, K., Kaminska, J., Grynberg, M., Urbanek, A., Ayscough, K. R., \& Zoladek, T. (2017). Amino acid substitution equivalent to human chorea-acanthocytosis I2771R in yeast Vps13 protein affects its binding to phosphatidylinositol 3-phosphate. Hum Mol Genet, 26(8), 1497-1510.

Saha, S., Panigrahi, D. P., Patil, S., \& Bhutia, S. K. (2018). Autophagy in health and disease: a comprehensive review. Biomed Pharmacother, 104, 485-495.

Sakoh-Nakatogawa, M., Matoba, K., Asai, E., Kirisako, H., Ishii, J., Noda, N. N., ... \& Ohsumi, Y. (2013). Atg12-Atg5 conjugate enhances E2 activity of Atg3 by rearranging its catalytic site. Nat Struct Mol Biol, 20(4), 433.

Salari, R., \& Salari, R. (2017). Investigation of the best Saccharomyces cerevisiae growth condition. Electron Physician, 9(1), 3592.

Sambrook, J., Fritsch, E. F., \& Maniatis, T. (1989). Molecular cloning: a laboratory manual. Cold spring harbor laboratory press.

Sankaram, M. B., \& Thompson, T. E. (1991). Cholesterol-induced fluid-phase immiscibility in membranes. Proc Natl Acad Sci U.S.A, 88(19), 8686-8690.

Sarkisyan, K. S., Goryashchenko, A. S., Lidsky, P. V., Gorbachev, D. A., Bozhanova, N. G., Gorokhovatsky, A. Y., ... \& Solntsev, K. M. (2015). Green fluorescent protein with anionic tryptophan-based chromophore and long fluorescence lifetime. Biophys J, 109(2), 380-389.

Schindelin, J., Arganda-Carreras, I., Frise, E., Kaynig, V., Longair, M., Pietzsch, T., ... \& Tinevez, J. Y. (2012). Fiji: an open-source platform for biological-image analysis. Nat Methods, 9(7), 676.

Schneiter, R., Hitomi, M., Ivessa, A. S., Fasch, E. V., Kohlwein, S. D., \& Tartakoff, A. M. (1996). A yeast acetyl coenzyme $A$ carboxylase mutant links very-long-chain fatty acid synthesis to the structure and function of the nuclear membrane-pore complex. Mol Cell Biol, 16(12), 7161-7172.

Schneiter, R., \& Kohlwein, S. D. (1997). Organelle structure, function, and inheritance in yeast: a role for fatty acid synthesis? Cell, 88(4), 431-434.

Schu, P. V., Takegawa, K., Fry, M. J., Stack, J. H., Waterfield, M. D., \& Emr, S. D. (1993). Phosphatidylinositol 3-kinase encoded by yeast VPS34 gene essential for protein sorting. Science, 260(5104), 88-91.

Schuck, S., Gallagher, C. M., \& Walter, P. (2014). ER-phagy mediates selective degradation of endoplasmic reticulum independently of the core autophagy machinery. J Cell Sci, 127(18), 4078-4088.

Scott, S. V., Nice, D. C., Nau, J. J., Weisman, L. S., Kamada, Y., Keizer-Gunnink, I., ... \& Klionsky, D. J. (2000). Apg13p and Vac8p are part of a complex of phosphoproteins that are required for cytoplasm to vacuole targeting. J Biol Chem, 275(33), 25840-25849.

Sekito, T., Kawamata, T., Ichikawa, R., Suzuki, K., \& Ohsumi, Y. (2009). Atg17 recruits Atg9 to organize the pre-autophagosomal structure. Genes Cells, 14(5), 525-538. 
Seong, E., Insolera, R., Dulovic, M., Kamsteeg, E. J., Trinh, J., Brüggemann, N., .. \& Jewett, T. (2018). Mutations in VPS13D lead to a new recessive ataxia with spasticity and mitochondrial defects. Ann Neurol, 83(6), 1075-1088.

Severs, N. J., Jordan, E. G., \& Williamson, D. H. (1976). Nuclear pore absence from areas of close association between nucleus and vacuole in synchronous yeast cultures. J Ultrastruct Res, 54(3), 374-387.

Shaner, N. C., Campbell, R. E., Steinbach, P. A., Giepmans, B. N., Palmer, A. E., \& Tsien, R. Y. (2004). Improved monomeric red, orange and yellow fluorescent proteins derived from Discosoma sp. red fluorescent protein. Nat Biotechnol, 22(12), 1567.

Sherman, F. (2002). Getting started with yeast. Methods Enzymol, 350, 3-41.

Shimizu, S., Takehara, T., Hikita, H., Kodama, T., Tsunematsu, H., Miyagi, T., ... \& Hiramatsu, N. (2012). Inhibition of autophagy potentiates the antitumor effect of the multikinase inhibitor sorafenib in hepatocellular carcinoma. Int J Cancer, 131(3), 548-557.

Shintani, T., Mizushima, N., Ogawa, Y., Matsuura, A., Noda, T., \& Ohsumi, Y. (1999). Apg10p, a novel protein-conjugating enzyme essential for autophagy in yeast. EMBO J, 18(19), 5234-5241.

Shintani, T., Suzuki, K., Kamada, Y., Noda, T., \& Ohsumi, Y. (2001). Apg2p functions in autophagosome formation on the perivacuolar structure. J Biol Chem, 276(32), 30452-30460.

Shirahama-Noda, K., Kira, S., Yoshimori, T., \& Noda, T. (2013). TRAPPIII is responsible for vesicular transport from early endosomes to Golgi, facilitating Atg9 cycling in autophagy. J Cell Sci, 126(21), 4963-4973.

Simon, H. U., Yousefi, S., Schmid, I., \& Friis, R. (2014). ATG5 can regulate p53 expression and activation. Cell Death Dis, 5(7), 1339.

Simonsen, A., Wurmser, A. E., Emr, S. D., \& Stenmark, H. (2001). The role of phosphoinositides in membrane transport. Curr Opin Cell Biol, 13(4), 485-492.

Smotrys, J. E., \& Linder, M. E. (2004). Palmitoylation of intracellular signaling proteins: regulation and function. Annu Rev Biochem, 73(1), 559-587.

Soubannier, V., McLelland, G. L., Zunino, R., Braschi, E., Rippstein, P., Fon, E. A., \& McBride, H. M. (2012). A vesicular transport pathway shuttles cargo from mitochondria to lysosomes. Curr Biol, 22(2), 135-141.

Stack, J. H., DeWald, D. B., Takegawa, K., \& Emr, S. D. (1995). Vesicle-mediated protein transport: regulatory interactions between the Vps15 protein kinase and the Vps34 Ptdlns 3-kinase essential for protein sorting to the vacuole in yeast. J Cell Biol, 129(2), 321-334.

Stack, J. H., \& Emr, S. D. (1994). Vps34p required for yeast vacuolar protein sorting is a multiple specificity kinase that exhibits both protein kinase and phosphatidylinositol-specific PI 3-kinase activities. J Biol Chem, 269(50), 31552-31562. 
Stasyk, O. V., Stasyk, O. G., Mathewson, R. D., Farré, J. C., Nazarko, V. Y., Krasovska, O. S., ... \& Sibirny, A. A. (2006). Atg28, a novel coiled-coil protein involved in autophagic degradation of peroxisomes in the methylotrophic yeast Pichia pastoris. Autophagy, 2(1), 30-38.

Steensels, J., Gorkovskiy, A., \& Verstrepen, K. J. (2018). SCRaMbLEing to understand and exploit structural variation in genomes. Nat Commun, 9(1), 1937.

Stephan, J. S., Yeh, Y. Y., Ramachandran, V., Deminoff, S. J., \& Herman, P. K. (2009). The Tor and PKA signaling pathways independently target the Atg1/Atg13 protein kinase complex to control autophagy. Proc Natl Acad Sci U.S.A, 106(40), 17049-17054.

Straub, M., Bredschneider, M., \& Thumm, M. (1997). AUT3, a serine/threonine kinase gene, is essential for autophagocytosis in Saccharomyces cerevisiae. J Bacteriol, 179(12), 3875-3883.

Subach, O. M., Gundorov, I. S., Yoshimura, M., Subach, F. V., Zhang, J., Grüenwald, D., ... \& Verkhusha, V. V. (2008). Conversion of red fluorescent protein into a bright blue probe. Chem Biol, 15(10), 1116-1124.

Suzuki, K., Akioka, M., Kondo-Kakuta, C., Yamamoto, H., \& Ohsumi, Y. (2013). Fine mapping of autophagy-related proteins during autophagosome formation in Saccharomyces cerevisiae. J Cell Sci, 126(11), 2534-2544.

Suzuki, K., Kirisako, T., Kamada, Y., Mizushima, N., Noda, T., \& Ohsumi, Y. (2001). The preautophagosomal structure organized by concerted functions of APG genes is essential for autophagosome formation. EMBO J, 20(21), 5971-5981.

Suzuki, K., Noda, T., \& Ohsumi, Y. (2004). Interrelationships among Atg proteins during autophagy in Saccharomyces cerevisiae. Yeast, 21(12), 1057-1065.

Suzuki, K., \& Ohsumi, Y. (2010). Current knowledge of the pre-autophagosomal structure (PAS). FEBS Lett, 584(7), 1280-1286.

Suzuki, S. W., Yamamoto, H., Oikawa, Y., Kondo-Kakuta, C., Kimura, Y., Hirano, H., \& Ohsumi, Y. (2015). Atg13 HORMA domain recruits Atg9 vesicles during autophagosome formation. Proc Natl Acad Sci U.S.A, 112(11), 3350-3355.

Takeshige, K., Baba, M., Tsuboi, S., Noda, T., \& Ohsumi, Y. (1992). Autophagy in yeast demonstrated with proteinase-deficient mutants and conditions for its induction. J Cell Biol, 119(2), 301-311.

Tamura, N., Oku, M., Ito, M., Noda, N. N., Inagaki, F., \& Sakai, Y. (2013). Atg18 phosphoregulation controls organellar dynamics by modulating its phosphoinositide-binding activity. J Cell Biol, 202(4), 685-698.

Tan, D., Cai, Y., Wang, J., Zhang, J., Menon, S., Chou, H. T., ... \& Walz, T. (2013). The EM structure of the TRAPPIII complex leads to the identification of a requirement for COPII vesicles on the macroautophagy pathway. Proc Natl Acad Sci U.S.A, 110(48), 19432-19437. 
Tanaka, C., Tan, L. J., Mochida, K., Kirisako, H., Koizumi, M., Asai, E., .. \& Nakatogawa, H. (2014). Hrr25 triggers selective autophagy-related pathways by phosphorylating receptor proteins. J Cell Biol, 207(1), 91-105.

Tang, F., Kauffman, E. J., Novak, J. L., Nau, J. J., Catlett, N. L., \& Weisman, L. S. (2003). Regulated degradation of a class $\mathrm{V}$ myosin receptor directs movement of the yeast vacuole. Nature, 422(6927), 87.

Tang, F., Peng, Y., Nau, J. J., Kauffman, E. J., \& Weisman, L. S. (2006). Vac8p, an armadillo repeat protein, coordinates vacuole inheritance with multiple vacuolar processes. Traffic, $7(10)$, 1368-1377.

Taylor, G. S., Maehama, T., \& Dixon, J. E. (2000). Myotubularin, a protein tyrosine phosphatase mutated in myotubular myopathy, dephosphorylates the lipid second messenger, phosphatidylinositol 3-phosphate. Proc Natl Acad Sci U.S.A, 97(16), 8910-8915.

Teichert, U., Mechler, B., Müller, H., \& Wolf, D. H. (1989). Lysosomal (vacuolar) proteinases of yeast are essential catalysts for protein degradation, differentiation, and cell survival. J Biol Chem, 264(27), 16037-16045.

Terpitz, U., Raimunda, D., Westhoff, M., Sukhorukov, V. L., Beaugé, L., Bamberg, E., \& Zimmermann, D. (2008). Electrofused giant protoplasts of Saccharomyces cerevisiae as a novel system for electrophysiological studies on membrane proteins. Biochimica et Biophysica Acta (BBA)-Biomembranes, 1778(6), 1493-1500.

Teter, S. A., Eggerton, K. P., Scott, S. V., Kim, J., Fischer, A. M., \& Klionsky, D. J. (2001). Degradation of lipid vesicles in the yeast vacuole requires function of $\mathrm{Cvt17}$, a putative lipase. $J$ Biol Chem, 276(3), 2083-2087.

Teuling, E., Ahmed, S., Haasdijk, E., Demmers, J., Steinmetz, M. O., Akhmanova, A., ... \& Hoogenraad, C. C. (2007). Motor neuron disease-associated mutant vesicle-associated membrane protein-associated protein (VAP) B recruits wild-type VAPs into endoplasmic reticulum-derived tubular aggregates. J Neurosci, 27(36), 9801-9815.

Tewari, R., Bailes, E., Bunting, K. A., \& Coates, J. C. (2010). Armadillo-repeat protein functions: questions for little creatures. Trends Cell Biol, 20(8), 470-481.

Thomas, A. C., Williams, H., Setó-Salvia, N., Bacchelli, C., Jenkins, D., O’Sullivan, M., ... \& James, C. (2014). Mutations in SNX14 cause a distinctive autosomal-recessive cerebellar ataxia and intellectual disability syndrome. Am J Hum Genet, 95(5), 611-621.

Thumm, M., Egner, R., Koch, B., Schlumpberger, M., Straub, M., Veenhuis, M., \& Wolf, D. H. (1994). Isolation of autophagocytosis mutants of Saccharomyces cerevisiae. FEBS Lett, 349(2), 275-280.

Tong, J., Manik, M. K., \& Im, Y. J. (2018). Structural basis of sterol recognition and nonvesicular transport by lipid transfer proteins anchored at membrane contact sites. Proc Natl Acad Sci U.S.A, 115(5), 856-865. 
Tong, J., Manik, M. K., Yang, H., \& Im, Y. J. (2016). Structural insights into nonvesicular lipid transport by the oxysterol binding protein homologue family. Biochimica et Biophysica Acta (BBA)-Molecular and Cell Biology of Lipids, 1861(8), 928-939.

Tong, J., Yang, H., Yang, H., Eom, S. H., \& Im, Y. J. (2013). Structure of Osh3 reveals a conserved mode of phosphoinositide binding in oxysterol-binding proteins. Structure, 21(7), 1203-1213.

Toulmay, A., \& Prinz, W. A. (2012). A conserved membrane-binding domain targets proteins to organelle contact sites. J Cell Sci, 125(1), 49-58.

Toulmay, A., \& Prinz, W. A. (2013). Direct imaging reveals stable, micrometer-scale lipid domains that segregate proteins in live cells. J Cell Biol, 202(1), 35-44.

Towbin, H., Staehelin, T., \& Gordon, J. (1979). Electrophoretic transfer of proteins from polyacrylamide gels to nitrocellulose sheets: procedure and some applications. Proc Natl Acad Sci U.S.A, 76(9), 4350-4354.

Treco, D. A., \& Winston, F. (2008). Growth and manipulation of yeast. Curr Protoc Mol Biol, 82(1), 13-2.

Tsukada, M., \& Ohsumi, Y. (1993). Isolation and characterization of autophagy-defective mutants of Saccharomyces cerevisiae. FEBS Lett, 333(1-2), 169-174.

Tuttle, D. L., \& Dunn, W. A. (1995). Divergent modes of autophagy in the methylotrophic yeast Pichia pastoris. J Cell Sci, 108(1), 25-35.

Uetz, P., Giot, L., Cagney, G., Mansfield, T. A., Judson, R. S., Knight, J. R., ... \& Qureshi-Emili, A. (2000). A comprehensive analysis of protein-protein interactions in Saccharomyces cerevisiae. Nature, 403(6770), 623.

Uttenweiler, A., Schwarz, H., Neumann, H., \& Mayer, A. (2007). The vacuolar transporter chaperone (VTC) complex is required for microautophagy. Mol Biol Cell, 18(1), 166-175.

Valente, G., Morani, F., Nicotra, G., Fusco, N., Peracchio, C., Titone, R., .. \& Isidoro, C. (2014). Expression and clinical significance of the autophagy proteins BECLIN 1 and LC3 in ovarian cancer. BioMed research international, 2014.

van den Hazel, H. B., Kielland-Brandt, M. C., \& Winther, J. R. (1996). Biosynthesis and function of yeast vacuolar proteases. Yeast, 12(1), 1-16.

van Zutphen, T., Todde, V., de Boer, R., Kreim, M., Hofbauer, H. F., Wolinski, H., ... \& Kohlwein, S. D. (2014). Lipid droplet autophagy in the yeast Saccharomyces cerevisiae. Mol Biol Cell, 25(2), 290-301.

Veit, M., Laage, R., Dietrich, L., Wang, L., \& Ungermann, C. (2001). Vac8p release from the SNARE complex and its palmitoylation are coupled and essential for vacuole fusion. EMBO J, 20(12), 3145-3155.

Velayos-Baeza, A., Vettori, A., Copley, R. R., Dobson-Stone, C., \& Monaco A. P. (2004). Analysis of the human VPS13 gene family. Genomics, 84(3), 536-549. 
Velikkakath, A. K. G., Nishimura, T., Oita, E., Ishihara, N., \& Mizushima, N. (2012). Mammalian Atg2 proteins are essential for autophagosome formation and important for regulation of size and distribution of lipid droplets. Mol Biol Cell, 23(5), 896-909.

Vevea, J. D., Garcia, E. J., Chan, R. B., Zhou, B., Schultz, M., Di Paolo, G., .. \& Pon, L. A. (2015). Role for lipid droplet biogenesis and microlipophagy in adaptation to lipid imbalance in yeast. Dev Cell, 35(5), 584-599.

Vollert, C. S., \& Uetz, P. (2004). The phox homology (PX) domain protein interaction network in yeast. Mol Cell Proteom, 3(11), 1053-1064.

Wang, C. W., Kim, J., Huang, W. P., Abeliovich, H., Stromhaug, P. E., Dunn, W. A., \& Klionsky, D. J. (2001). Apg2 is a novel protein required for the cytoplasm to vacuole targeting, autophagy, and pexophagy pathways. J Biol Chem, 276(32), 30442-30451.

Wang, C. W., Miao, Y. H., \& Chang, Y. S. (2014). A sterol-enriched vacuolar microdomain mediates stationary phase lipophagy in budding yeast. J Cell Biol, 206(3), 357-366.

Wang, C. W., Stromhaug, P. E., Kauffman, E. J., Weisman, L. S., \& Klionsky, D. J. (2003). Yeast homotypic vacuole fusion requires the Ccz1-Mon1 complex during the tethering/docking stage. $J$ Cell Biol, 163(5), 973-985.

Wang, C. W., Stromhaug, P. E., Shima, J., \& Klionsky, D. J. (2002). The Ccz1-Mon1 protein complex is required for the late step of multiple vacuole delivery pathways. $J$ Biol Chem, 277(49), 47917-47927.

Wang, J., Davis, S., Menon, S., Zhang, J., Ding, J., Cervantes, S., ... \& Ferro-Novick, S. (2015). Ypt1/Rab1 regulates Hrr25/CK1 $\delta$ kinase activity in ER-Golgi traffic and macroautophagy. J Cell Biol, 210(2), 273-285.

Wang, J., Menon, S., Yamasaki, A., Chou, H. T., Walz, T., Jiang, Y., \& Ferro-Novick, S. (2013). Ypt1 recruits the Atg1 kinase to the preautophagosomal structure. Proc Natl Acad Sci U.S.A, 110(24), 9800-9805.

Wang, T. Y., \& Silvius, J. R. (2003). Sphingolipid partitioning into ordered domains in cholesterolfree and cholesterol-containing lipid bilayers. Biophys J, 84(1), 367-378.

Wang, Y. X., Catlett, N. L., \& Weisman, L. S. (1998). Vac8p, a vacuolar protein with armadillo repeats, functions in both vacuole inheritance and protein targeting from the cytoplasm to vacuole. J Cell Biol, 140(5), 1063-1074.

Wang, Y. X., Kauffman, E. J., Duex, J. E., \& Weisman, L. S. (2001). Fusion of docked membranes requires the armadillo repeat protein Vac8p. J Biol Chem, 276(37), 35133-35140.

Weidberg, H., Shvets, E., Shpilka, T., Shimron, F., Shinder, V., \& Elazar, Z. (2010). LC3 and GATE16/GABARAP subfamilies are both essential yet act differently in autophagosome biogenesis. EMBO J, 29(11), 1792-1802. 
Weiss, B., \& Richardson, C. C. (1967). Enzymatic breakage and joining of deoxyribonucleic acid, I. Repair of single-strand breaks in DNA by an enzyme system from Escherichia coli infected with T4 bacteriophage. Proc Natl Acad Sci U.S.A, 57(4), 1021.

Woolford, C. A., Daniels, L. B., Park, F. J., Jones, E. W., Van Arsdell, J. N., \& Innis, M. A. (1986). The PEP4 gene encodes an aspartyl protease implicated in the posttranslational regulation of Saccharomyces cerevisiae vacuolar hydrolases. Mol Cell Biol, 6(7), 2500-2510.

Wu, Y., Cheng, S., Zhao, H., Zou, W., Yoshina, S., Mitani, S., ... \& Wang, X. (2014). PI3P phosphatase activity is required for autophagosome maturation and autolysosome formation. EMBO Rep, 15(9), 973-981.

Wurm, C. A., Kolmakov, K., Göttfert, F., Ta, H., Bossi, M., Schill, H., ... \& Hell, S. W. (2012). Novel red fluorophores with superior performance in STED microscopy. Opt Nanoscopy, 1(1), 7.

Xia, H. G., Najafov, A., Geng, J., Galan-Acosta, L., Han, X., Guo, Y., ... \& Pan, L. (2015). Degradation of HK2 by chaperone-mediated autophagy promotes metabolic catastrophe and cell death. J Cell Biol, 210(5), 705-716.

Xie, Q., Tzfadia, O., Levy, M., Weithorn, E., Peled-Zehavi, H., Van Parys, T., ... \& Galili, G. (2016). hfAIM: A reliable bioinformatics approach for in silico genome-wide identification of autophagyassociated Atg8-interacting motifs in various organisms. Autophagy, 12(5), 876-887.

Xie, W., Zhang, L., Jiao, H., Guan, L., Zha, J., Li, X., ... \& You, H. (2015). Chaperone-mediated autophagy prevents apoptosis by degrading BBC3/PUMA. Autophagy, 11(9), 1623-1635.

Yamada, T., Carson, A. R., Caniggia, I., Umebayashi, K., Yoshimori, T., Nakabayashi, K., \& Scherer, S. W. (2005). Endothelial nitric-oxide synthase antisense (NOS3AS) gene encodes an autophagyrelated protein (APG9-like2) highly expressed in trophoblast. J Biol Chem, 280(18), 18283-18290.

Yamaguchi, M., Noda, N. N., Yamamoto, H., Shima, T., Kumeta, H., Kobashigawa, Y., ... \& Inagaki, F. (2012). Structural insights into Atg10-mediated formation of the autophagy-essential Atg12Atg5 conjugate. Structure, 20(7), 1244-1254.

Yamamoto, H., Fujioka, Y., Suzuki, S. W., Noshiro, D., Suzuki, H., Kondo-Kakuta, C., ... \& Ohsumi, Y. (2016). The intrinsically disordered protein Atg13 mediates supramolecular assembly of autophagy initiation complexes. Dev Cell, 38(1), 86-99.

Yamamoto, H., Kakuta, S., Watanabe, T. M., Kitamura, A., Sekito, T., Kondo-Kakuta, C., ... \& Ohsumi, Y. (2012). Atg9 vesicles are an important membrane source during early steps of autophagosome formation. J Cell Biol, 198(2), 219-233.

Yamashita, S. I., Oku, M., \& Sakai, Y. (2007). Functions of PI4P and Sterol Glucoside Necessary for the Synthesis of a Nascent Membrane Structure During Pexophagy. Autophagy, 3(1), 35-37.

Yamashita, S. I., Oku, M., Wasada, Y., Ano, Y., \& Sakai, Y. (2006). PI4P-signaling pathway for the synthesis of a nascent membrane structure in selective autophagy. J Cell Biol, 173(5), 709-717.

Yang, A., Li, Y., Pantoom, S., Triola, G., \& Wu, Y. W. (2013). Semisynthetic lipidated LC3 protein mediates membrane fusion. ChemBioChem, 14(11), 1296-1300. 
Yang, Q., She, H., Gearing, M., Colla, E., Lee, M., Shacka, J. J., \& Mao, Z. (2009). Regulation of neuronal survival factor MEF2D by chaperone-mediated autophagy. Science, 323(5910), 124-127.

Yang, T. T., Cheng, L., \& Kain, S. R. (1996). Optimized codon usage and chromophore mutations provide enhanced sensitivity with the green fluorescent protein. Nucleic Acids Res, 24(22), $4592-4593$.

Yankiwski, V., Marciniak, R. A., Guarente, L., \& Neff, N. F. (2000). Nuclear structure in normal and Bloom syndrome cells. Proc Natl Acad Sci U.S.A, 97(10), 5214-5219.

Yen, W. L., Shintani, T., Nair, U., Cao, Y., Richardson, B. C., Li, Z., ... \& Klionsky, D. J. (2010). The conserved oligomeric Golgi complex is involved in double-membrane vesicle formation during autophagy. J Cell Biol, 188(1), 101-114.

Ylä-Anttila, P., Vihinen, H., Jokitalo, E., \& Eskelinen, E. L. (2009). 3D tomography reveals connections between the phagophore and endoplasmic reticulum. Autophagy, 5(8), 1180-1185.

Yorimitsu, T., \& Klionsky, D. J. (2005). Autophagy: molecular machinery for self-eating. Cell Death Differ, 12(S2), 1542.

Young, A. R., Chan, E. Y., Hu, X. W., Köchl, R., Crawshaw, S. G., High, S., ... \& Tooze, S. A. (2006). Starvation and ULK1-dependent cycling of mammalian Atg9 between the TGN and endosomes. $J$ Cell Sci, 119(18), 3888-3900.

Yuan, W., Tuttle, D. L., Shi, Y. J., Ralph, G. S., \& Dunn, W. A. (1997). Glucose-induced microautophagy in Pichia pastoris requires the alpha-subunit of phosphofructokinase. J Cell Sci, 110(16), 1935-1945.

Yue, Z., Jin, S., Yang, C., Levine, A. J., \& Heintz, N. (2003). Beclin 1, an autophagy gene essential for early embryonic development, is a haploinsufficient tumor suppressor. Proc Natl Acad Sci U.S.A, 100(25), 15077-15082.

Zhang, T., Péli-Gulli, M. P., Yang, H., de Virgilio, C., \& Ding, J. (2012). Ego3 functions as a homodimer to mediate the interaction between Gtr1-Gtr2 and Ego1 in the ego complex to activate TORC1. Structure, 20(12), 2151-2160. 


\section{Acknowledgements}

Foremost, I would like to thank Michael Thumm. Providing me with the opportunity to work on this challenging, but ultimately rewarding project. I have not only benefitted from the nature of your supervision but attained insights that helped me comprehend both science and academia. Thank you for entrusting me with the freedom to frequently follow my curiosity and your enthusiasm for novel approaches. I am grateful for your advice, discussions and guidance.

I have benefited considerably from having Markus Bohnsack and Silvio Rizzoli be part of my thesis advisory committee. Thank you for your time, your suggestions and the encouragement towards helping me put my work and doubts into a realistic perspective. I would also like to express my gratitude to Stefan Jakobs, Roland Dosch and Ralf Kehlenbach for agreeing to join my extended examination board.

I would like to thank Peter Rube for helping me get started in the workgroup. I missed that a lot. Peter and Ridhima thank you for endlessly troubleshooting all aspects of this work and beyond. This thesis would not be the same without you.

I am very grateful for the opportunity of doing STED microscopy. I would like to thank Silvio Rizzoli for helping me understand the intricacies of fluorescence microscopy and the perspective for new methods, as well as Felipe Opazo and Manuel Maidorn for introducing me to the STED setup.

Thank you to all members of the Thumm group, past and present for the time in the lab and outside. Thank you Lisa, Petra, Rosi, Lena, Stefanie, Peter, Matt, Hussein and Lisa. Thank you Michael, Peter and Olaf for mensa lunches. Petra, Danke für's Tauchen und all deine Unterstützung. Lena for macroautophagy 
mornings, Lisa for coffee chitchat, Matt for early cinnamon lunch therapy and Hussein for evening hope towards the future. Danke Lisa, Lisa und Marcel für DoKo.

I would like to extend my thanks to all members of the institute of cellular biochemistry. Welcoming me into the institute (thank you Tobi) was a unique experience and I feel very lucky having felt included here (thank you Natasha).

Nine years of Göttingen. Rayan, Joris, Joni, Cici. I'm very glad I met you.

Danke an meine Familie, danke für all eure Unterstützung.

Nini. Danke, dass du da warst.

Danke an meine Eltern. Ich hatte immer alle Hilfe und Unterstützung von euch. Danke für all das was Ihr mir ermöglicht habt.

Danke Märill. Sieben Jahre. Danke für dich. Und dein endloses Verständnis.

Nina, die Letzte ist für dich. 